\title{
Evidence for WZ Production and a Measurement of the WZ Production Cross Section
}

\author{
by \\ James D. Degenhardt \\ A dissertation submitted in partial fulfillment \\ of the requirements for the degree of \\ Doctor of Philosophy \\ (Physics) \\ in The University of Michigan \\ 2007
}

Doctoral Committee:

Professor Bing Zhou, Chair

Emeritus Professor Henry C. Griffin

Professor Christopher R. Monroe

Professor Jianming Qian

Professor Rudolf P. Thun

Associate Professor James T. Liu 
(C) James D. Degenhardt

2007 


\section{DEDICATION}

To my family, especially my parents Karen and Robert Degenhardt. 


\section{ACKNOWLEDGEMENTS}

I would like to acknowledge those that have aided me in and enriched my educational career. Obtaining my Ph.D. was not easy and it has been a long time in coming, but it would not have been possible had it not been for many people who have supported me. I can not possibly acknowledge everyone, but I will attempt to do so nonetheless.

First and foremost, I would like to acknowledge my mother and father. With out their love and support and timely encouragements this would have never come to fruition, period. I attribute my success to my parents. I also would like to acknowledge my academic parent, Bing Zhou. She introduced me to high energy physics, and ever since then I never looked back. She has been very supportive and has aided me when I needed it most. She is the best advisor there is.

I would like to recognize Henry Griffin for introducing me to experimental scientific research. The experiences that I had under his supervision have proven to be priceless. I would also like to thank the rest of my doctoral committee for taking time out of their precious schedules to be on my committee. James Liu, Christopher Monroe, Jianming Qian, and Rudolf Thun, I very much appreciate their work.

I need to also acknowledge Andrew Alton, Andrew Askew, and Tom Diehl. These gentlemen worked with me on my thesis research and their advice, arguments, and contributions have all been key ingredients to completing this dissertation. This is not to say that the rest of the $\mathrm{D} \varnothing$ collaboration is not worth recognizing. When I came to the 
collaboration the experiment was up and running and already making important contributions to science. Without the valuable work from the hundreds of physicists that came before me, my work would not have been possible.

While doing my course work at Michigan, I feel that I should also recognize the priceless advice that I obtained while working alongside the ATLAS technicians, Dave Kouba, Helmut Schick, and Curtis Weaverdyck. I will always remember the morning coffees and friday afternoon beers. The stories will stay with me forever. The most important thing I learned from these fine gentlemen have been that a lifetime of experience will always be worth more than a fancy education. Edward Diehl, Daniel Levin, and Zhengguo Zhao are the research scientists that I worked with during my undergraduate work and they helped me learn how high energy research is done.

I would like to thank my family and friends who's support helped to make these past few challenging years bearable. First there is Kimberly Smith, Dianne Ziesmer, Matt Blank, and Elizabeth Branch, without who I would have never made it. Together those four make up the best academic services ever. There is also my friends who I have known since elementary school. Kowalis, Miller, Bear, Sticker, Phil, Nathan, Matt Miller, all of you have been so supportive. 


\section{TABLE OF CONTENTS}

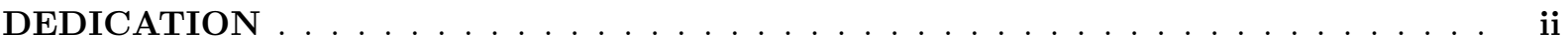

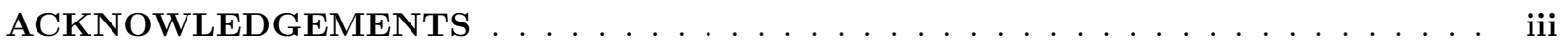

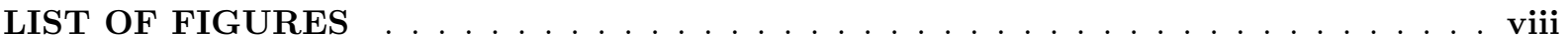

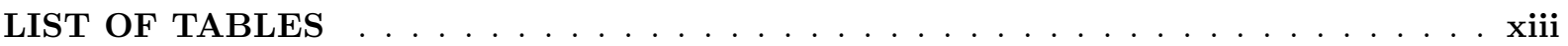

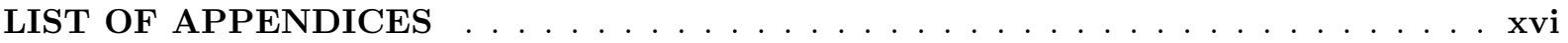

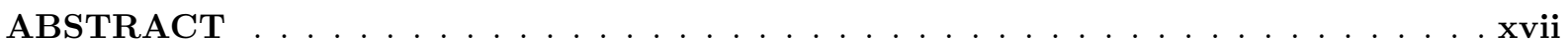

CHAPTER

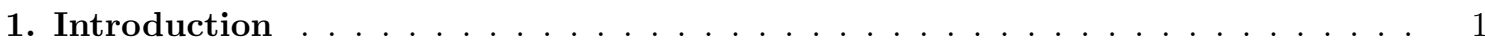

2. The Standard Model and the Physics of $W Z$ Production . . . . . . . . . . 12

2.1 The Standard Model and Particle Physics . . . . . . . . . . . . . . . . . . . . 12

2.2 Electroweak Interactions . . . . . . . . . . . . . . . . . . . . . . 14

$2.3 W Z$ Production in the Standard Model . . . . . . . . . . . . . . . . . 17

2.3.1 WZ Production Mechanisms . . . . . . . . . . . . . . . . . 18

2.3.2 Standard Model Predictions for $W Z$ Production . . . . . . . . . . . . 18

2.3.3 Experimental Signature of $W Z$ Production . . . . . . . . . . . . 20

2.4 Summary of the Theoretical Prediction . . . . . . . . . . . . . . . . . . 22

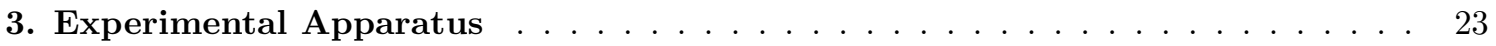

3.1 The Accelerators . . . . . . . . . . . . . . . . . . . . 23

3.1.1 The Cockroft Walton Pre-Accelerator . . . . . . . . . . . . . . 25

3.1 .2 Linac . . . . . . . . . . . . . . . . . . . . . . . . 25

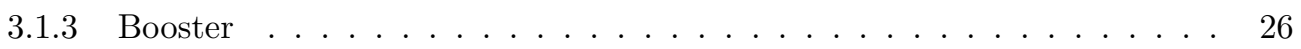

3.1 .4 Main Injector . . . . . . . . . . . . . . . . . . . . . . . 28

3.1.5 Antiproton Source . . . . . . . . . . . . . . . . . . . 28

3.1 .6 Recycler . . . . . . . . . . . . . . . . . . . . . . . . . . . . 29

3.1 .7 Tevatron . . . . . . . . . . . . . . . . . . . . . 31

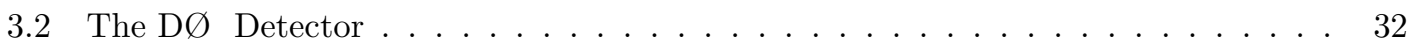

3.2.1 The D $\varnothing$ Coordinate System . . . . . . . . . . . . . . . . 32

3.3 Luminosity Monitor . . . . . . . . . . . . . . . . . . . . . . . 35

3.3.1 The Central Tracking System . . . . . . . . . . . . . . . 37

3.3 .2 The Solenoid . . . . . . . . . . . . . . . . . . . . . 38

3.3.3 The Silicon Microstrip Tracker Detector . . . . . . . . . . . . . . . . 39

3.3.4 The Central Fiber Tracker Detector . . . . . . . . . . . . . . . . . . 42

3.3.5 The Preshower Detectors . . . . . . . . . . . . . . . . . . . . . . 42 
3.3.6 Central Preshower ....................... 44

3.3.7 Forward Preshower . . . . . . . . . . . . . . . . . . . . 46

3.3 .8 The Calorimeters . . . . . . . . . . . . . . . . . . . 46

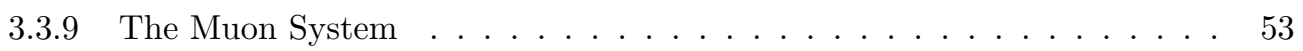

4. The D $\emptyset$ Trigger and Data Acquisition Systems . . . . . . . . . . . . . . . . . 57

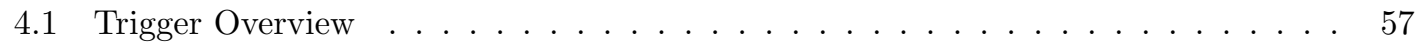

4.2 The Level-1 Trigger . . . . . . . . . . . . . . . . . . . . . . . . . . 58

4.2.1 The Trigger Framework . . . . . . . . . . . . . . . . . . . 59

4.2.2 The Level-1 Calorimeter Trigger . . . . . . . . . . . . . . . . . . . . 59

4.2 .3 The Level-1 Central Track Trigger . . . . . . . . . . . . . . . . . . . 59

4.2.4 The Level-1 Muon Trigger . . . . . . . . . . . . . . . . . . . . 60

4.3 The Level-2 Trigger . . . . . . . . . . . . . . . . . . . . . . . . 60

4.3.1 Level-2 Global Processor . . . . . . . . . . . . . . . . . . 60

4.3.2 Level-2 Calorimeter Preprocessor . . . . . . . . . . . . . . . . . 61

4.3.3 Level-2 Silicon Track Trigger Preprocessors . . . . . . . . . . . . . . . . 62

4.3.4 Level-2 Central Track Trigger Preprocessor . . . . . . . . . . . . . . . 62

4.3.5 Level-2 Preshower Preprocessor . . . . . . . . . . . . . . . . . 62

4.3.6 Level-1 Muon Preprocessor . . . . . . . . . . . . . . . . 63

4.4 Level-3 Trigger and Data Acquisition . . . . . . . . . . . . . . . . . . . 63

5. Offline Event Reconstruction and Object Identification . . . . . . . . . . . . . . . 64

5.1 Track Reconstruction . . . . . . . . . . . . . . . . . . . . . . . 64

5.2 Primary Vertex Reconstruction . . . . . . . . . . . . . . . . . . . . 66

5.3 Electromagnetic Object Reconstruction . . . . . . . . . . . . . . . . . . . . 68

5.4 Jet Reconstruction and Identification . . . . . . . . . . . . . . . . . . . . 70

5.4 .1 The Jet Reconstruction Algorithm . . . . . . . . . . . . . . . . . . 70

5.4 .2 Jet Identification . . . . . . . . . . . . . . . . . . . 73

5.5 Muon Reconstruction . . . . . . . . . . . . . . . . . . . . . . . . 74

6. Data Samples and Luminosity . . . . . . . . . . . . . . . . . . . . 76

7. Data Analysis . . . . . . . . . . . . . . . . . . . . . . . 81

7.0 .1 Lepton Identification . . . . . . . . . . . . . . . . . . 81

7.0.2 Vector Boson Selection Criteria . . . . . . . . . . . . . . . . 84

7.0 .3 Additional Signal Criteria .................. 86

7.1 Efficiency ............................ 88

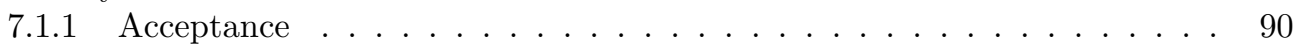

7.1 .2 Electron Efficiency . . . . . . . . . . . . . . . . . . . . . . 92

7.1.3 Muon Efficiency . . . . . . . . . . . . . . . . . . . . 92

7.1.4 Total Acceptance times Efficiency . . . . . . . . . . . . . . . . 94

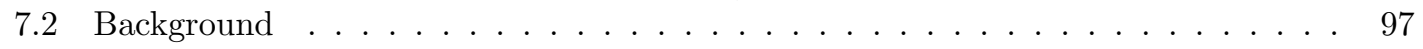

7.2.1 QCD Backgrounds ................... 98

7.2.2 QCD Background Cross Check ................. 103

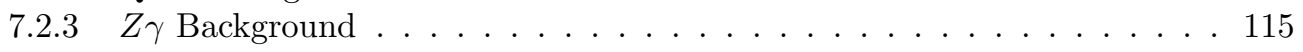

$7.2 .4 Z Z$ Background ....................... 117

7.2.5 $t \bar{t}$ Background . . . . . . . . . . . . . . . . . . . . . 119

7.2 .6 Drell-Yan Background . . . . . . . . . . . . . . . . 121

7.2 .7 Total Background . . . . . . . . . . . . . . . . . 122 
7.3 Systematics . . . . . . . . . . . . . . . . . . . . . . 122

8. Cross Section Measurement and WZ Gauge Coupling Measurement . . . . . 128

8.1 Cross section . . . . . . . . . . . . . . . . . . . . 128

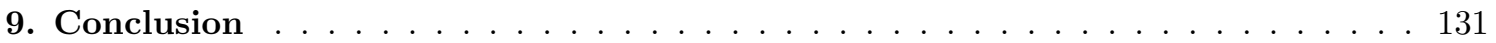

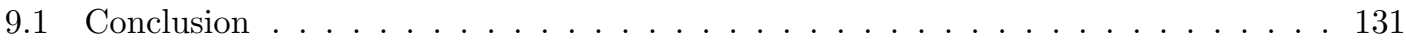

APPENDICES . . . . . . . . . . . . . . . . . . . . . . . . 132

BIBLIOGRAPHY . . . . . . . . . . . . . . . . . . . . . . . . . 159 


\section{LIST OF FIGURES}

\section{Figure}

1.1 The $t$ - (a) and $s$ - (b) channel tree level Feynman diagrams for $W Z$ production at the Tevatron. The $s$ channel contains that $W W Z$ vertex that allows us to directly measure the coupling strengths of the massive vector bosons to each other. If an anomalous signal is seen then there must be contributing interactions to the $W Z$ final state that are not accounted for in the SM. . . . . . . . . . . . . . . . . . . . . . . 11

2.1 Feynman diagrams of the trilinear gauge boson couplings allowed by the Standard Model. The $W W Z$ vertex is shown in (a) while the $W W \gamma$ vertex is shown in (b). . . . . . . . 17

2.2 Standard Model Feynman Diagrams for tree level $W Z$ production with subsequent decay into leptons: (a) $u$-channel, (b) $t$-channel, (c) $s$-channel. . . . . . . . . . . . . . . . 19

3.1 The schematic view of the accelerator at Fermilab. The Recycler is housed above the Main Injector. The Debuncher and Accumulator are in the Antiproton Source ring. . . .

3.2 Hand drawing of the $\mathrm{H}^{-}$ion source or magnetron. $\ldots \ldots \ldots \ldots$

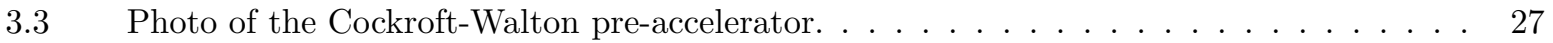

$3.4 \quad$ A drawing of the Linac RF cavity. . . . . . . . . . . . . . . . . . . . 27

3.5 A schematic of the antiproton generating nickel target. . . . . . . . . . . . . . 29

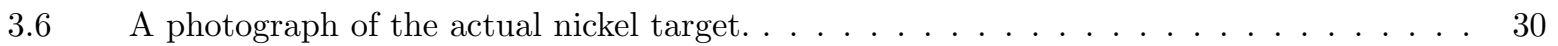

$3.7 \quad$ A photograph of the Lithium lens showing the beam's eye view. . . . . . . . . . . . 30

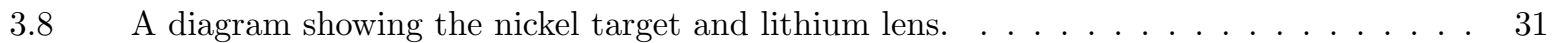

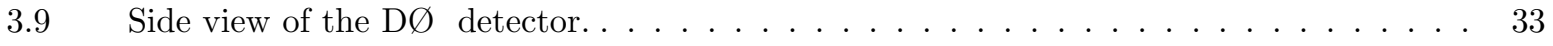

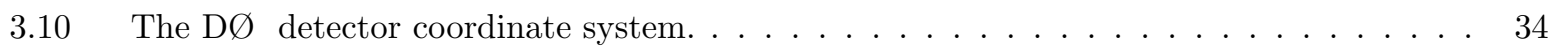

3.11 A diagram of the $\mathrm{D} \emptyset$ luminosity monitors. The $r-\phi$ view is shown on the left, while the $r-z$ view of the two monitors is shown on the right. The photomultiplier tubes are represented by small circles on each tile. . . . . . . . . . . . . . . . 35

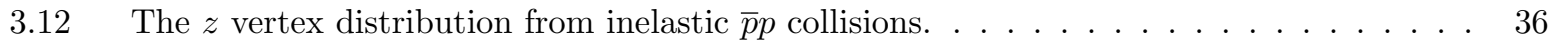

3.13 The total amount of luminosity delivered to and recorded by the $\mathrm{D} \varnothing$ detector. . . . . 37

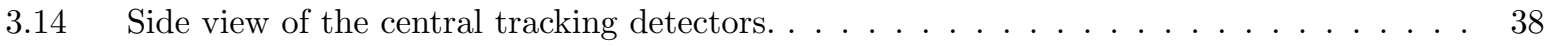


3.15 The magnetic field lines extend out to the magnetic toroid. The strength of the magnetic

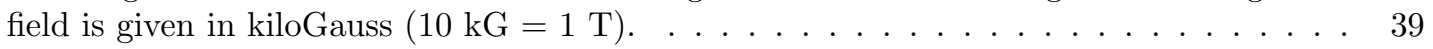

3.16 A 3D layout of the Silicon Microstrip Tracker. . . . . . . . . . . . . . . . 40

3.17 The $r-\phi$ view of the silicon barrels. . . . . . . . . . . . . . . . . . . 41

3.18 The $r-\phi$ view of the silicon wedge detectors of the F-disks. . . . . . . . . . . . . 41

3.19 The central fiber tracker located between the silicon microstrip tracker and the solenoid. The cross section blow up of the fibers from two layers. . . . . . . . . . . . . . . . . . 43

3.20 a) The $r-z$ diagram of the central fiber tracker. b) A cross section view of the fibers. . 43

3.21 Schematic $r-z$ side view of the central preshower detector. . . . . . . . . . . . 45

3.22 Schematic $r-\phi$ view of the central preshower detector. . . . . . . . . . . . . 45

3.23 The cross section of the central and forward preshower scintillating triangular strips. . . 45

3.24 The layout of the three layers of scintillating strips - one axial and two at stereo angles. 46

3.25 The forward preshower detectors attached to each of the calorimeter end caps. $\quad . \quad$. . 47

3.26 A view of the central and end cap calorimeters. . . . . . . . . . . . . . . . . . 49

3.27 Schematic view of a unit cell in the calorimeter. . . . . . . . . . . . . . . . 50

3.28 One quarter view of the calorimeter showing the segmented readout. . . . . . . . . 51

3.29 The calorimeter readout towers in $\eta$ versus the layers distributed along the shower de-

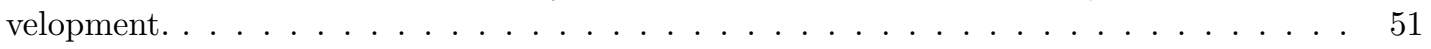

3.30 The shape of the signals detected and processed through the electronics in the calorimeter. 52

3.31 The electronic readout of the unit cell to the analog to digital converter. . . . . . . . 53

3.32 Cross section through proportional drift chambers. . . . . . . . . . . . . . . . . 54

3.33 A cross section of the mini-drift tubes. . . . . . . . . . . . . . . . . . 54

3.34 Simulated muon trajectory through the D $\varnothing$ detector. . . . . . . . . . . . 56

4.1 The flow of the data from the $\mathrm{D} \varnothing$ detector to storage onto magnetic tapes. . . . . . . 58

4.2 The flow of the data from the L1 inputs to the Level-2 trigger. . . . . . . . . . . . . 61

5.1 The decay paths of quarks and gluons. Quarks and gluons, after final state radiation, form parton jets. Hadrons and leptons form particle jets after hadronization. Energy deposits in the calorimeter are reconstructed as calorimeter jets. . . . . . . . . . . . 71

7.1 The ee invariant mass distribution of the signal data sample before the analysis mass cuts are applied. The fits used are a Gaussian convoluted with a Lorentzian which are plotted on top of an exponential fit. The exponential fit is to model the background. . . 
7.2 The $\mu \mu$ invariant mass distribution in the signal data sample before the analysis mass cuts are applied. The fits used are a Gaussian convoluted with a Lorentzian which are plotted on top of an exponential fit. The exponential fit is to model the background. .

7.3 Shown is the dilepton mass of the $Z$ boson pair from each candidate event along with the background and MC signal overlaid. . . . . . . . . . . . . . . . 88

7.4 Shown is the distribution of the transverse mass of the third lepton and $\mathbb{E}_{T}$ along with the background and $\mathrm{MC}$ signal overlaid. . . . . . . . . . . . . . .

7.5 Shown is the dilepton invariant mass versus $\mathbb{E}_{T}$ of the candidate events along with the sum of the background estimates and expected signal. The MC are normalized to the data luminosity. . . . . . . . . . . . . . . . . . . . . . .

7.6 Shown are the isolation efficiencies for muons in data and MC. The muon isolation efficiencies are plotted bins of physics $\eta \ldots \ldots \ldots \ldots \ldots$

7.7 Shown are the isolation efficiency corrections for muons MC. The muon isolation efficiency corrections are plotted bins of physics $\eta \ldots \ldots \ldots \ldots \ldots$. . . . . . . . . 94

7.8 The electron fake probability as a function of detector $\eta$. The error bars are statistical error bars. . . . . . . . . . . . . . . . . . . . . . . . . 101

7.9 The eee QCD fake background vs. detector $\eta$ determined from the ee+ jets sample. The error bars are statistical error bars. . . . . . . . . . . . . . . . . . . . . . 101

7.10 The $\mu \mu e$ QCD fake background vs.detector $\eta$ determined from the $\mu \mu+$ jets sample. The error bars are statistical error bars. . . . . . . . . . . . . . . . . . . 102

7.11 The ee $\mu$ QCD fake background vs.detector $\eta$ determined from the $e+\mu+$ jets sample.

The error bars are statistical error bars. . . . . . . . . . . . . . . . . . . 102

7.12 Distribution of good electron $p_{T}$ in the QCD sample. . . . . . . . . . . 104

7.13 Distribution of bad electron $p_{T}$ in the QCD sample. . . . . . . . . . 105

7.14 Distribution of good electrons in detector $\eta$ in the QCD sample. . . . . . . . 106

7.15 Distribution of bad electron detector $\eta$ in the QCD sample. . . . . . . . . 106

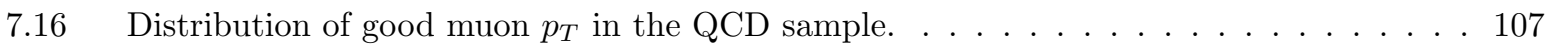

7.17 Distribution of the bad muon $p_{T}$ in the QCD sample. . . . . . . . . . . 107

7.18 Distribution of the good muon detector $\eta$ in the QCD sample. . . . . . . . . . 108

7.19 Distribution of the bad muon detector $\eta$ in the QCD sample. . . . . . . . . . . 108

7.20 Distribution of electron fake rate in $p_{T}$. The error bars are the statistical Gaussian uncertainties. . . . . . . . . . . . . . . . . . . . . 109

7.21 Distribution of electron fake rate in detector $\eta$.The error bars are the statistical Gaussian uncertainties. . . . . . . . . . . . . . . . . . . . . 109

7.22 Distribution of muon fake rate in $p_{T}$. The error bars are the statistical Gaussian uncer-

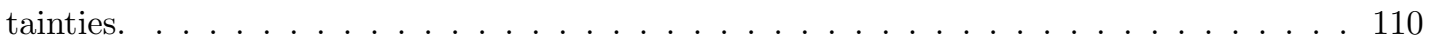


7.23 Distribution of muon fake rate in detector $\eta$.The error bars are the statistical Gaussian uncertainties. . . . . . . . . . . . . . . . . . . 110

7.24 Normalization sample of $e e+$ bad $e$ events distributed in $p_{T} \ldots \ldots \ldots \ldots \ldots$

7.25 Normalization sample of $e e+$ bad $e$ events distributed in detector $\eta$. . . . . . . . 111

7.26 Normalization sample of $e e+$ bad $\mu$ events distributed in $p_{T} \ldots \ldots \ldots 112$

7.27 Normalization sample of $e e+$ bad $\mu$ events distributed in detector $\eta$. . . . . . . . 112

7.28 Normalization sample of $\mu \mu+$ bad $e$ events distributed in $p_{T} \ldots \ldots \ldots \ldots$

7.29 Normalization sample of $\mu \mu+$ bad $e$ events distributed in detector $\eta$. . . . . . . . 113

$7.30 \quad$ Normalization sample of $\mu \mu+$ bad $\mu$ events distributed in $p_{T} \ldots \ldots \ldots \ldots \ldots$

7.31 Normalization sample of $\mu \mu+$ bad $\mu$ events distributed in detector $\eta$. . . . . . . 115

7.32 Normalized background estimate for the eee channel distributed in $p_{T}$. This distribution yields $0.278 \pm 0.133$ events. This distribution is determined by multiplying the distribution in Fig. 7.20 by the distribution in Fig. $7.24 \ldots \ldots \ldots \ldots$

7.33 Normalized background estimate for the eee channel distributed in detector $\eta$. This distribution yields $0.213 \pm 0.115$ events. This distribution is determined by multiplying the distribution in Fig. 7.21 by the distribution in Fig. 7.25 . . . . . . . . . . . 117

7.34 Normalized background estimate for the $e e \mu$ channel distributed in $p_{T}$. This distribution yields $0.152 \pm 0.044$ events. This distribution is determined by multiplying the distribution in Fig. 7.22 by the distribution in Fig. $7.26 \ldots \ldots \ldots \ldots$. . . . . . . . . 118

7.35 Normalized background estimate for the ee $\mu$ channel distributed in detector $\eta$. This distribution yields $0.128 \pm 0.055$ events. This distribution is determined by multiplying the distribution in Fig. 7.23 by the distribution in Fig. 7.27 . . . . . . . . . . . . 119

7.36 Normalized background estimate for the $\mu \mu e$ channel distributed in $p_{T}$. This distribution yields $0.684 \pm 0.288$ events. This distribution is determined by multiplying the distribution in Fig. 7.20 by the distribution in Fig. 7.28 . . . . . . . . . . . . . . . . . 120

7.37 Normalized background estimate for the $\mu \mu e$ channel distributed in detector $\eta$. This distribution yields $1.016 \pm 0.428$ events. This distribution is determined by multiplying the distribution in Fig. 7.21 by the distribution in Fig. 7.29 . . . . . . . . . . . . 121

7.38 Normalized background estimate for the $\mu \mu \mu$ channel distributed in $p_{T}$. This distribution yields $0.196 \pm 0.092$ events. This distribution is determined by multiplying the distribution in Fig. 7.22 by the distribution in Fig. 7.30 . . . . . . . . . . . . . . . 122

7.39 Normalized background estimate for the $\mu \mu \mu$ channel distributed in detector $\eta$. This distribution yields $0.197 \pm 0.093$ events. This distribution is determined by multiplying the distribution in Fig. 7.23 by the distribution in Fig. 7.31 . . . . . . . . . . . . 123

7.40 Shown are the three body masses of the $Z \gamma$ events used by the photon ID group. The black histogram are the events where the $\gamma$ passed the electron likelihood criteria. The red histogram are the events where the $\gamma$ failed the electron likelihood criteria. The photons in these samples also passed a threshold cut of $15 \mathrm{GeV}$. The resulting efficiency is $0.042 \pm 0.015$. The uncertainty is the statistical uncertainty. . . . . . . . . . . 123 
7.41 Presented is the ratio of selected electrons to generated photons in a sample of $\mu \mu+\gamma$ initial state radiation pythia MC that has been simulated using the D $\varnothing$ Geant detector simulation. The error bars are statistical uncertainties. The line is the average ratio, $0.06 \pm 0.003$. A systematic error on the ratio is determined by the highest and lowest bin in the sample resulting in an uncertainty of $0.029 \ldots \ldots \ldots$. . . . . . . . . 124

8.1 Combined negative log likelihood as a function of cross section. Arrows indicate the points 0.5 units of likelihood above the minimum. . . . . . . . . . . . . 130

B.1 The $\mathbb{E}_{T}$ versus invariant mass distributions of the sample that did not pass the $M_{\ell \ell}$ cuts. 136

B.2 The $\mathbb{E}_{T}$ distribution of the sample that did not pass the $M_{\ell \ell}$ cuts. . . . . . . 136

B.3 The invariant mass distribution of the sample that did not pass the $M_{\ell \ell}$ cuts. . . . . 137

B.4 The invariant mass distribution of the sample that did not pass the $\mathbb{E}_{T}$ cut. No invariant mass cut was applied in this sample. . . . . . . . . . . . . . . . 137

C.1 Shown is the $E_{T}$ of the candidate events along with the sum of the background estimates and expected signal. The MC are normalized to the data luminosity. . . . . . . . . . . 139

E.1 Event View: Run Number - 191882 Event Number - 21112860 . . . . . . . . . . . . . . 146

E.2 Event View: Run Number - 195167 Event Number - 18587081 . . . . . . . . . . . . . 147

E.3 Event View: Run Number - 194259 Event Number - 5929362 . . . . . . . . . . . . . . 148

E.4 Event View: Run Number - 205114 Event Number - 3409480 . . . . . . . . . . . . . . . 149

E.5 Event View: Run Number - 207094 Event Number - 10178395 . . . . . . . . . . . . . . 150

E.6 Event View: Run Number - 204938 Event Number - 6268052 . . . . . . . . . . . . . . 151

E.7 Event View: Run Number - 204318 Event Number - 69485771 . . . . . . . . . . . . . . . 152

E.8 Event View: Run Number - 206332 Event Number - 20605317 . . . . . . . . . . . . . . 153

E.9 Event View: Run Number - 210156 Event Number - 248377216 . . . . . . . . . . . . . 154

E.10 Event View: Run Number - 207769 Event Number - 23761167 . . . . . . . . . . . . . . 155

E.11 Event View: Run Number - 207596 Event Number - 12955559 . . . . . . . . . . . . 156

E.12 Event View: Run Number - 188371 Event Number - 23177216 . . . . . . . . . . . . . . 157

E.13 Event View: Run Number - 192868 Event Number - 66474158 . . . . . . . . . . . . 158 


\section{LIST OF TABLES}

\section{$\underline{\text { Table }}$}

3.1 Location of silicon microstrip tracker components along the $z$ direction starting from the center of the detector. . . . . . . . . . . . . . . . . . . . . . . . . . . . . . . . 41

3.2 Silicon microstrip tracker detector component radii (measured from the center of the

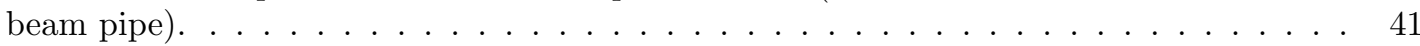

3.3 Presented are the calorimeter detector parameter values. . . . . . . . . . . . . . . 49

3.4 Presented are the end calorimeter parameters. . . . . . . . . . . . . . . 50

6.1 This table shows the integrated luminosity for the 2EMhighpt data sample. The run ranges and integrated luminosity for the major trigger versions are shown. Along with the current luminosity is listed the luminosity that is used for the preliminary measurement as well as the ratio of the two luminosity constants. The data that did not need re-fixing is listed under the v14(b) data epoch. The cable swap data, v14(c), is included but was not included until after the new luminosity constant and therefore does not have an old luminosity for comparison. The total numbers are for the old luminosity (up to v14(b)) and all of the new luminosity. All of the luminosity numbers have an associated $6.5 \%$

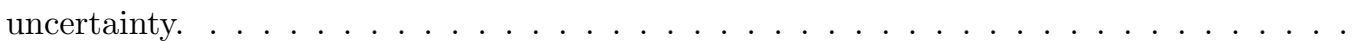

6.2 This table shows the integrated luminosity for the 2EMhighpt data sample with muon quality cuts applied. The run ranges and integrated luminosity for the major trigger versions are shown. Along with the current luminosity is listed the luminosity that is used for the preliminary measurement as well as the ratio of the two luminosity constants. The data that did not need re-fixing is listed under the v14(b) data epoch. The cable swap data, v14(c), is included but was not included until after the new luminosity constant and therefore does not have an old luminosity for comparison. The total numbers are for the old luminosity (up to v14(b)) and all of the new luminosity. The ratio is calculated before applying the muon quality cuts. All of the luminosity numbers have an associated $6.5 \%$ uncertainty. . . . . . . . . . . . . . . . . . . .

6.3 This table shows the integrated luminosity for the 2MUhighpt data sample. The run ranges and integrated luminosity for the major trigger versions are shown. Along with the current luminosity is listed the luminosity that is used for the preliminary measurement as well as the ratio of the two luminosity constants. The data that did not need re-fixing is listed under the v14(b) data epoch. The cable swap data, v14(c), is included but was not included until after the new luminosity constant and therefore does not have an old luminosity for comparison. The total numbers are for the old luminosity (up to v14(b)) and all of the new luminosity. All of the luminosity numbers have an associated $6.5 \%$

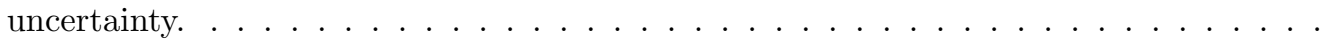


7.1 Acceptance of $W Z$ diboson events for different trilepton decay channels. The acceptance is calculated based on the kinematic and the geometric cuts. The uncertainties on the acceptances are purely statistical. . . . . . . . . . . . . . . . . . . .

7.2 Presented are the total efficiencies, including the trigger and charge identification efficiencies, with uncertainties (stat. + syst.) along with the expected number of signal events and their uncertainties broken down into signal decay channel. The number of $\mathrm{MC}$ candidates used in determining the acceptance times efficiency is shown. The efficiency is presented when only the decay from $W$ and $Z$ bosons are directly to leptons ( $e$ or $\mu$ ) are taken into account. Then the efficiency of the case where a boson decays to one or two $\tau$ 's which decay directly to leptons is also presented. Finally the total acceptance times efficiency including $\tau$ decays is presented. . . . . . . . . . . . . . .

7.3 Presented in this table are the branching fractions for the massive vector bosons to decay to electrons or muons and for the $\tau$ lepton to decay to an electron or muon. Also listed are the four branching fractions for the four channels. The branching fractions are taken

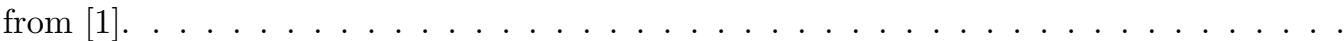

7.4 Summarized in this table are the values of the parameters used in determining the number of expected $Z$ bosons in the dilepton decay channels. . . . . . . . . . . . . .

7.5 In this table the $Z \rightarrow \ell \ell$ event yields in data are compared with expectations determined using Equations 7.6 and 7.7. The systematic uncertainties on the data are taken from the uncertainties on the background subtraction. . . . . . . . . . . . .

7.6 Presented in this table is the breakdown of the uncertainty in the expected $Z$ boson yield in the dilepton decay channels. The uncertainties are shown in percentages and in events. 98

7.7 Shown are the background estimates from QCD using jets and bad leptons. The previous estimate used jets as a way to estimate the QCD contribution to the $W Z \rightarrow \ell \nu \ell \ell$ final state. The cross check uses bad leptons in jet triggered events to perform the same estimate. The uncertainties for the previous estimate are the combined statistical and systematic uncertainties. The uncertainties on the cross check values are the statistical and systematic uncertainties respectively. The statistical uncertainties on the cross check values are approximated as Poisson errors. The systematics uncertainties on the cross check is estimated by taking the difference of the normalized backgrounds distributed in $p_{T}$ to those distributed in $\eta \ldots \ldots \ldots \ldots \ldots \ldots \ldots$. . . . . . . . . . . . . . . . . . . . .

7.8 The $Z \gamma$ background is estimated using MC events from SM PYTHIA. The backgrounds are listed in the table. . . . . . . . . . . . . . . . . . . . . 124

7.9 $Z Z$ background contribution. The uncertainties are the combined systematic and statistical uncertainties. . . . . . . . . . . . . . . . . . . . 125

$7.10 t \bar{t}$ background. The uncertainties are the combined systematic and statistical uncertainties.125

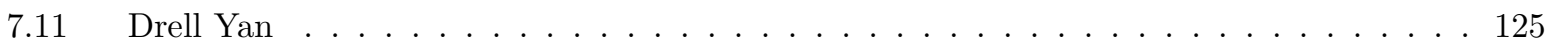

7.12 Estimated background broken down by signal decay channel. The uncertainty on the backgrounds are the combined statistical and systematic errors. . . . . . . . . . . 126

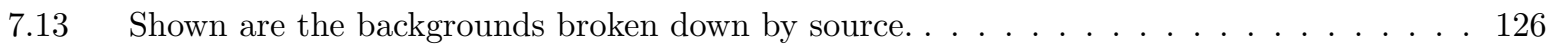


7.14 Presented is a breakdown of the systematics contributing the uncertainty on the number of signal events expected. The first source, cross section, in the table is broken down into its PDF and scale sources. . . . . . . . . . . . . . . . . . . . . 127

7.15 Presented are the dominant systematics, broken down by channel, that contribute to the number of background events, and ultimately contribute to the systematic uncertainty in the cross section determination. The QCD source is the contribution from the QCD normalization samples. The $f_{e}$ and $f_{m}$ are the contributions from the lepton fake rates from jets. The $\epsilon_{e}$ and $\epsilon_{\mu}$ are the contributions from the lepton ID efficiencies. . . . . . . 127

8.1 Summary of probability of background to fluctuate to the observed number of candidates, and significance by channel. . . . . . . . . . . . . . . . .

A.1 This table shows the cut flows for the data and signal MC. The cut flows start from the third lepton cut. The data also has the separation cut applied before the third lepton cut as this cut removes many events that contain two lepton that share the same track. The cause of this is speculated to be $\mu$ 's radiating bremsstrahlung radiation which gets reconstructed as an electron. This effect is not modeled well in the detector simulation therefore it would not show up in the MC samples. . . . . . . . . . . . . . . . . . 133

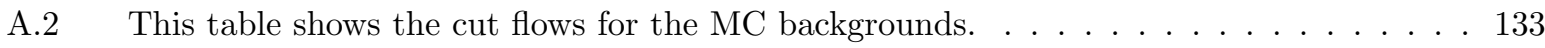

A.3 This table shows the cut flows for the $t \bar{t}$ backgrounds. . . . . . . . . . . . . . . 133

A.4 This table shows the cut flows for the Drell Yan backgrounds. . . . . . . . . . . . . 134

A.5 This table shows the cut flows for the QCD backgrounds. . . . . . . . . . . . . 134

C.1 This table shows the expected significance after applying a $M_{T}$ cut is applied to the data samples. The uncertainty on the background in this study is scaled with the background events. The significance is determined from the probability which the background would fluctuate to background plus expected signal as prescribed by D $\varnothing \ldots \ldots$. . . . . . . 138

C.2 This table shows the expected significance if the $\mathbb{E}_{T}$ cut is increased in $5 \mathrm{GeV}$ increments. The uncertainty on the background in this study is kept at 0.57 events. The significance is determined from the probability which the background would fluctuate to background plus expected signal as prescribed by $\mathrm{D} \varnothing \ldots \ldots \ldots$. . . . . . . . . . . . 139

C.3 This table shows the expected significance if the dilepton invariant mass cut is tightened. The uncertainty on the background in this study is scaled with the background. The significance is determined from the probability which the background would fluctuate to background plus expected signal as prescribed by $\mathrm{D} \emptyset \ldots \ldots \ldots$. . . . . . . . . . 140

E.1 Presented are the kinematic properties of each lepton for all of the candidate events. . . 144

E.2 Presented are the vector boson properties for each candidate event. One event has zero transverse mass because the $\phi$ of the $\mathbb{E}_{T}$ and the $e$ are the same, with in resolution effects, and therefore reconstruct a zero $M_{T} \ldots \ldots \ldots \ldots \ldots \ldots \ldots \ldots \ldots$ 


\section{LIST OF APPENDICES}

\section{$\underline{\text { Appendix }}$}

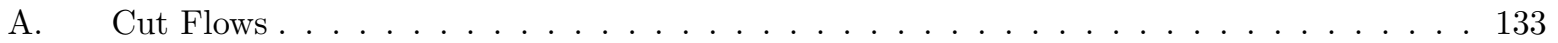

B. Control Sample . . . . . . . . . . . . . . . . . . . . . . . 135

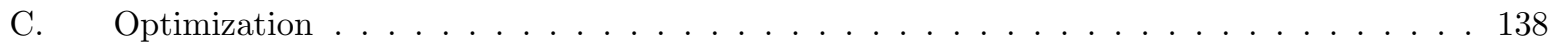

D. $\quad$ Trigger List . . . . . . . . . . . . . . . . . . . . . . 141

E. Candidate List . . . . . . . . . . . . . . . . . . . . . . . . . . . 144 


\begin{abstract}
Evidence for WZ Production and a Measurement of the WZ Production Cross Section

by

James D. Degenhardt
\end{abstract}

Chair: Bing Zhou

This dissertation describes a test of the Standard Model (SM) of particle physics by measuring the probability, or cross section, of simultaneously producing a $W$ boson and a $Z$ boson from proton-antiproton collisions. The SM predicts the cross section of $W Z$ production to be $3.68 \pm 0.25 \mathrm{pb}$. The $\mathrm{SM}$ and physics of $W Z$ production are described in Chapter 2 of this dissertation. The $1.96 \mathrm{TeV}$ center-of-mass energy proton-antiproton collisions are provided by the Fermi National Accelerator Laboratory (FNAL) Tevatron Collider. The $W$ and $Z$ particles are detected using the D $\varnothing$ detector, which is described in Chapter 3. The data were collected by the detector during 2002-2006 corresponding to $1 \mathrm{fb}^{-1}$ of $p \bar{p}$ collisions. This data set is described in Chapter 6 . The measurement uses the trilepton (evee, $\mu \nu e e, e \nu \mu \mu$, and $\mu \nu \mu \mu)$ decay channels, in which a $W$ decays to a charged lepton plus a neutrino and a $Z$ decays to a pair of charged leptons. The $W$ and $Z$ particle selection criteria, detection efficiency, and background determination are described in Chapter 7 . We observe 13 candidate events in $1 \mathrm{fb}^{-1}$ of $p \bar{p}$ collisions. In this data set we expect to see $4.5 \pm 0.6$ background events, and we expect to see $9.2 \pm 1.0$ signal events. The probability of $4.5 \pm 0.6$ background events to fluctuate to 13 or more events is $1.2 \times 10^{-3}$ which is a $3.0 \sigma$ deviation from the background estimate. A log 
likelihood method is used to determine the most likely cross section as determined by the measured signal efficiencies, the expected backgrounds, and the observed data. Presented in Chapter 8 is a measurement of the cross section for $p \bar{p} \rightarrow W Z+X$ at $\sqrt{s}=1.96 \mathrm{TeV}$. The $W Z$ diboson production cross section is measured to be $\sigma_{W Z}=2.7_{-1.3}^{+1.7} \mathrm{pb}$. This is in agreement with the predicted Standard Model cross section. 


\section{CHAPTER 1}

\section{Introduction}

Particle physics is the field of physics that studies the phenomena of subatomic particles. Since the dawn of time man has pondered what the fundamental constituents of matter are. Particle physics, or high energy physics, tries to find these fundamental constituents and tries to understand how these particles interact. Through many successful theoretical developments, this field, as it is now understood, is described by the Standard Model (SM) theory of fundamental particles [2]. For over thirty years this theory has been tested, and subsequently it has prevailed and as a result it has become the corner stone of particle physics. It describes all of the known fundamental particles and their interactions with great success. Still there remain unanswered questions that the Standard Model cannot answer. For example, there is the question of why neutrinos have mass, and why is that mass so small compared to other particles? How do all of the fundamental particles acquire mass, and why do they have the spectrum of masses that is seen in na-

ture? Also, what is this mysterious phenomena known as dark matter? Experimentalists search for clues to the answers of these questions by looking for new phenomena, such as new particles, or by looking for inconsistencies between the SM and nature, in essence by testing the SM. This dissertation describes a test of the Standard Model theory of parti- 
cle physics. It tests the SM by detailing a measurement of the probability of producing simultaneous $W$ and $Z$ bosons from colliding protons $(p)$ and anti-protons $(\bar{p})$. $W$ and $Z$ bosons are particles that were discovered in the 1980's [3], [4], [5], [6]. The SM requires that $W$ and $Z$ bosons be produced simultaneously and at a particular non-zero, yet small, probability. These predictions are a direct result of the symmetry in which the theory is based upon, and therefore if the predictions are unconfirmed then the premiss of the SM theory must be incorrect. This particular production of simultaneous $W$ and $Z$ bosons, also known as $W Z$ diboson production, has never before been observed by the $\mathrm{D} \varnothing$ collaboration. This dissertation describes the measurement of the $W Z$ diboson production cross section from $p \bar{p}$ collisions at a center-of-mass energy of 1.96 Terraelectronvolts (TeV) by the $\mathrm{D} \varnothing$ detector.

Most of modern elementary particle physics is based on the study and tests of the SM. The Standard Model is the combination of three successful quantum field theories that have been developed over the last fifty to sixty years. The most famous of these quantum field theories is Quantum Electrodynamics (QED). Richard Feynman is considered to be the father of QED [7]. QED deals mainly with the fundamental interactions of photons, or light, with electrons. It was Steven Weinberg, Sheldon Glashow and Abdus Salam who built upon QED and combined, or unified, it with the weak theory of particles, which describes the weak force, into one theory of electroweak particle physics. The weak theory of particles deals with interactions of the fundamental particles with $W$ and $Z$ bosons which mediate the weak force, much like photons mediate the electric force. The final component of the SM is the strong theory of particles which describes the strong force and how it interacts with particles. This is commonly referred to as Quantum Chromodynamics (QCD) because the charge associated with the strong force is referred to as color. QCD was championed by David Politzer, Franck Wilczek, and David Gross. 
The modern treatment of quantum field theory is to assume a symmetry of the theory as fundamental and then build the theory from this assumption. The symmetries of the theories then lead to invariance laws inherit in the theory. This was first shown by Emmy Neother [8] in 1918. Today the mathematical constructs known as groups are used as guides in developing quantum field theories. Certain groups poses symmetries that can be readily translated into symmetries of quantum field theories. QED is often referred to a $U(1)$ theory, where $U(1)$ describes the set of groups with the same symmetry. The symmetry of $U(1)$ leads to gauge invariance in QED. Weak theory can be described as an $S U(2)$ theory and QCD can be described as an $S U(3)$ theory. $S U(2)$ symmetry of weak theory leads to the weak isospin invariance. $S U(3)$ symmetry leads to color invariance of QCD. A general description of group theory and its relation to the Standard Model of particle physics can be found in [2]. The SM in its most basic form describes the interactions of 16 fundamental particles. These 16 particles are divided into three categories. One category are the leptons, another category are the quarks, and the third category are the bosons. There are six leptons, which are divided into three families, the electron and electron neutrino $\left(e, \nu_{e}\right)$, the muon and muon neutrino $\left(\mu, \nu_{\mu}\right)$, and the tau and tau neutrino $\left(\tau, \nu_{\tau}\right)$. There are six quarks, which are also divided into three families, the up and down quark $(u, d)$, the strange and charm quarks $(s, c)$ and the top and bottom quarks $(t, b)$. The bosons are made up of the force mediating particles. The SM describes three fundamental forces. The electromagnetic force is mediated by the photon $(\gamma)$. The weak force, which is associated with beta decay, is mediated by the $W$ and $Z$ bosons. The strong force, which is also known as the nuclear force, is mediated by the gluon $(g)$. The fourth fundamental force, gravity, is not described by the SM. This dissertation will aim to communicate its discussion in this modern treatment of particle physics, which is mathematical, and concise in nature. It is common to construct what is 
called a Lagrangian equation which is used to describe field theories. The mathematical objects in a quantum field theory will interact with each other by manipulating energy in various ways. This manipulation of energy among the field theory objects is described by the Lagrangian. Thus the Standard Model can be thought of as a Lagrangian with an $S U(3) \times S U(2) \times U(1)$ structure [2].

The $S U(2) \times U(1)$ structure of the Standard Model (SM) Lagrangian implies that the electroweak gauge bosons, the $W$ and the $Z$, interact with one another through trilinear and quadrilinear vertices. There are two ways of producing $W Z$ dibosons at $\mathrm{D} \varnothing$. The first is by radiating two bosons from a quark as seen in Figure 1.1a. The second is by the radiation of a $Z$ boson off of a $W$ boson as seen in Figure 1.1b. This figure also shows the trilinear vertex of the $W W Z$ interaction which is a fundamental requirement by the SM. As a consequence, the production cross section $\sigma(p \bar{p} \rightarrow W Z)$ depends on the $W W Z$ gauge coupling shown in Figure 1.1b. The Standard Model predicts that the strength of that coupling, $g_{W W Z}$, is $-e \cot \theta_{W}$, where $e$ is the electron charge and $\theta_{W}$ is the weak mixing angle. Excursions of these $W W Z$ couplings from the SM can be described by an effective Lagrangian [9]

$$
\frac{\mathcal{L}_{W W Z}}{g_{W W Z}}=i g_{1}^{Z}\left(W_{\mu \nu}^{\dagger} W^{\mu} Z^{\nu}-W_{\mu}^{\dagger} Z_{\nu} W^{\mu \nu}\right)+i \kappa_{Z} W_{\mu}^{\dagger} W_{\nu} Z^{\mu \nu}+i \frac{\lambda_{Z}}{M_{W}^{2}} W_{\rho \mu}^{\dagger} W_{\nu}^{\mu} Z^{\nu \rho}
$$

where $W_{\mu \nu}=\partial_{\mu} W_{\nu}-\partial_{\nu} W_{\mu}$. The parameters $g_{1}^{Z}, \kappa_{Z}$ and $\lambda_{Z}$ describe the coupling strengths between the vector bosons. In the SM $g_{1}^{Z}=1, \kappa_{Z}=1, \lambda=0$, thus anomalous SM couplings defined as $\Delta g_{1}^{Z} \equiv g_{1}^{Z}-1, \Delta \kappa_{Z} \equiv \kappa_{Z}-1, \Delta \lambda_{Z} \equiv \lambda_{Z}$ will lead to an increase in the $W Z$ production cross section, $\sigma_{W Z}$, therefore this cross section is sensitive to new physics beyond the SM.

In order to test these models experimentalists need to produce enough energy in a small volume so that both $W$ and $Z$ bosons can be produced. Experimentalists also need to be 
able to probe distances that are much smaller than the diameter of a proton. Experimental particle physicists scatter high energy beams of particles off of other particles to probe ever smaller and smaller distances. The spatial resolution available to a scattering beam is inversely proportional to the energy of the beam [10]. To probe a very short distance, a very high energy is required. There are two methods of scattering beams. One is to scatter a beam of particles off of a stationary target of heavy nuclei. The other is to scatter one beam off of another beam. The stationary target method provides a centerof-mass energy of $E_{\mathrm{cm}} \approx \sqrt{2 E_{1} m_{2}}$, where $E_{\mathrm{cm}}$ is the center-of-mass energy, $E_{1}$ is the energy of the beam, and $m_{2}$ is the mass of the target nuclei. This says that the energy available at the center-of-mass is proportional to the square root of the energy of the beam. If we collide two beams however, the center-of-mass energy available is linearly proportional to the energy of the beams, $E_{\mathrm{cm}} \approx \sqrt{4 E_{1} E_{2}}$ where $E_{1}$, and $E_{2}$ are the energy of the beams that are colliding. Colliding two energetic beams rather than using a fixed target to collide a beam into, provides a larger center-of-mass energy therefore maximizes the energy reach of the collider. Energy reach is important for two reason. One is to be able to probe small distnaces. The other is to produce massive particles. In order to produce massive particles a collider needs to produce enough energy at the collision. This practice of colliding particles at higher and higher energies takes advantage of the Einstein matter-energy equivalence theorem $E=m c^{2}$, where $c$ is the speed of light in a vacuum. This means that if enough energy, $E$, is provided then it is possible to create a particle of rest mass $m$. For a more complete treatment of basics collider concepts the reader is directed to see reference [11].

The Fermi National Accelerator Laboratory (FNAL) Tevatron collider is the highest energy collider ever built [12]. The sole purpose of the Tevatron is to collide protons with anti-protons at the highest energies possible. Therefore the Tevatron provides a 
unique opportunity to measure the $W Z$ production cross section because it is the only collider with a large enough center-of-mass energy to produce bothe $W$ and $Z$ bosons and also allows for the charged intermediate propagator seen in the $s$-channel exchange of Figure 1.1b. The Tevatron collider is a large synchrotron storage ring. That is to say, it is a ring of magnets, about 3 miles in circumference, used to store high energy, or relativistic, protons and anti-protons. The design is based off of the race track design of other synchrotron rings that came before it. This design takes advantage of the Lorentz force law of charged particles, $F=q \vec{v} \times \vec{B}+q \vec{E}$. This equation tells us that when a charged particle with electronic charge $q$ and velocity $\vec{v}$ passes through a magnetic field which is perpendicular to its direction of motion, then that direction of motion will be deflected, and also that when a charged particle experiences an electric field, $\vec{E}$, the particle will accelerate in the direction of $\vec{E}$. So a ring of magnets is used to steer the protons and antiprotons around the ring, and at one point in the ring the particles are accelerated through an electric field. Picking up more energy each time they pass through that electric field. This design allows the Tevatron to continually collide a store of protons and anti-protons. For a more complete treatment of manipulating charged particles in synchrotrons the reader is encouraged to read reference [13].

Two experimental collaborations, the Collider Detector Facility (CDF), and DØ, have built large multipurpose detectors at two separate sections of the collider where the beam of protons and the beam of anti-protons are forced to collide. These intersection points are referred to as $\mathrm{B} \varnothing$, where $\mathrm{CDF}$ is located, and $\mathrm{D} \emptyset$, where the $\mathrm{D} \emptyset$ detector is located. It is these particle detectors that search for new particles, new event processes, and provide means to test new theories or to test the Standard Model. Though the detectors of both collaborations have distinctly different detectors they both have designs that use similar concepts. The basic strategy of a multipurpose high energy particle detector is to detect 
high momentum particles that originate from the $p \bar{p}$ collisions provided by the Tevatron. The detectors are built around the interaction point, with different layers of detectors. At the very center is a high resolution tracking detector that detects charged particles. High spatial resolution is needed so that the vertex of the colliding particles can be accurately meausred. The next layer of detectors is a momentum spectrometer. These detectors measure the momentum of charged particles in a magnetic field using the principle of the Lorentz force law. Outside of the momentum spectrometer is a series of calorimeters. A calorimeter is simply a detector that measures the amount of energy that a particle has. A calorimeter measures the energy of a particle by absorbing most, if not all, of the energy of a particle. So calorimeters will essentially stop and absorb all of the particles that come from the interaction point. That is unless the particles are a neutrino or a muon. So the final layer of detectors is the muon spectrometer. This is a series of tracking detectors that only see charged particles, and measures the momentum of those charged particles. The neutrino is not charged and so it is the only type of particle that will not be detected by a particle detector. Fortunately physicists can use the principles of momentum conservation and energy conservation to infer that a neutrino was created in an event. Once an event is detected it needs to be recorded. Not every event gets recorded because the Tevatron produces many interactions. An interaction happens about once every 396 seconds, and the collider runs essentially 24 hours a day and 7 days a week. This produces an enormous amount of data. Unfortunately a lot of the data is not very interesting. So the detectors contain a triggering system that decides which event is interesting and which event is not. This decision process is done on an event by event basis, so it has to be very fast. Each collaboration determines which events are special and should be recorded to data tape, and which events are not interesting and are thrown away. Since these detectors are multipurpose detectors they record a diverse set of events. Millions of events have 
been recorded and that accounts for a fraction of the total number of events that have occurred. The estimated number of signal events relevant to this dissertation that are in the recorded sample is about ten events. So the signal that is searched for in this dissertation is truly a rare signal.

The Tevatron is in its second run of operation, commonly referred to as Run II. In Run I the Tevatron used water cooled electro-magnets to collide protons and anti-protons. The center-of-mass energy $(\sqrt{s})$ was $1.8 \mathrm{TeV}$. During Run I both experimental collaborations are credited with discovering the top quark. After the completion of Run I the Tevatron and both experiments upgraded their technologies to improve their scientific reach. The Tevatron replaced its electromagnets with superconducting magnetics and can now reach a $\sqrt{s}$ of $1.96 \mathrm{TeV}$. The other major improvement to the Tevatron was the increase in instantaneous luminosity from $1 \times 10^{31} \mathrm{~cm}^{-2} / \mathrm{s}$ to $1 \times 10^{32} \mathrm{~cm}^{-2} / \mathrm{s}$. In experimental particle physics two key elements are required to search for rare events and massive fundamental objects. Those two elements are higher luminosity and higher energy.

Luminosity is commonly thought of as a measure of brightness. So in particle physics, light is thought of as photons, and the brightness of a source of light is thought of as the number of photons hitting an area per second. At the Tevatron we count number of events per unit time per square $\mathrm{cm}$. The beams that are colliding are divided into compact bunches of particles. The frequency that the bunches cross is called the interaction frequency and this is about $3 \mathrm{MHz}$. Each time the bunches cross they have a certain probability of interacting and creating an event. So the number of events per unit time is the bunch frequency times the number of interactions per crossing. This is divided by the effective cross section of the proton. The effective cross section of the proton is $60.7 \pm 2.4$ $\mathrm{mb}$ [14], or $6.07 \pm 0.24 \times 10^{-26} \mathrm{~cm}^{2}$. This is done using special detectors that are described later in Chapter 3. If the Tevatron luminosity is integrated over time, then we are left 
with the units of inverse cross section. When particle production rates are measured, we are measuring the amount of $p \bar{p}$ collisions required to produce a certain particle. This is referred to as a production cross section $\left(\sigma_{\text {process }}\right)$. In particle physics the unit of barn (b) is commonly used as the unit of cross section. One barn is equal to $10^{-24} \mathrm{~cm}^{2}$. Therefore integrated luminosity is measured in $\mathrm{b}^{-1}$ and hence the smaller the integrated luminosity the better the chances a collider has at producing rare events [11].

This dissertation approaches the cross section measurement by searching for detector events that contain the same characteristics as the expected signal events. This is done by looking at simulations of the signal events and how these will look in the detector. We then use the detector to look only for events with the unique set of event characteristics that describe the rare signal events. These characteristics are three charged leptons with large momentum as well as large missing Energy. Once the event characteristics are determined, then the measurement reduces to a counting experiment. Not every event will have three charged leptons and a large amount of missing energy. Most of the events that the detector sees can be discarded. What is left is a small amount of events that have the characteristics of the signal. Even though most of the events that do not come from the signal source have been excluded, some of the events that have the signal characteristics will have come from other physics sources. These types of events are categorized as background events. At this point the amount of selected data that comes from background is determined. Various methods are used to estimate how much of the events in the data may be accounted for by background events. The detector will not know which events are background and which are signal, but since the background events come from known sources we can use our knowledge of these sources to estimate precisely what amount of the data will originate from the background. This is explained in detail in Chapter 7.

Finally a maximum likelihood method is used to determine the most likely cross section 
for the process being investigated. Assuming a Poisson distribution, the likelihood is defined as

$$
L=-\ln \frac{s(\sigma)^{n} e^{-s(\sigma)}}{n !}
$$

where $s(\sigma)$ is a function of the cross section that is being measured and $n$ is the number of events found in the data. The Poisson distribution describes well the statistics of small numbers and is a good distribution to use because this dissertation describes the study of rare occurring events. The details of this measurement method are found in Chapter 8 . For a more complete discussion on the maximum likelihood method and poisson statistics see reference [15].

During the Tevatron Run I at the D $\varnothing$ experiment an upper limit $\sigma(p \bar{p} \rightarrow W Z)$ at the $95 \%$ confidence level (CL) was set at $47 \mathrm{pb}$ [16] after searching through $90 \mathrm{pb}^{-1}$ of data. The theoretical prediction of $W Z$ production at the Tevatron with $\sqrt{s}=1.8 \mathrm{TeV}$ is $2.6 \mathrm{pb}$. At the Tevatron Run II, at $\sqrt{s}=1.96 \mathrm{TeV}$, using approximately $0.3 \mathrm{fb}^{-1}$ of integrated luminosity data, we found 3 candidate events with $0.71 \pm 0.08$ events estimated to be background [17]. The probability for $0.71 \pm 0.08$ background events to fluctuate to 3 or more events is $3.6 \%$ (this is a significance greater than $1.8 \sigma$ ) and the cross section for this measurement was $4.5_{-2.6}^{+3.8} \mathrm{pb}$. An upper limit of $13.3 \mathrm{pb}$ was set at the $95 \% \mathrm{CL}$ on the cross section [17]. The CDF collaboration, during the Tevatron Run II, looked for both $W Z$ and $Z Z$ decays to $\ell \ell$, $\ell \ell \ell$, and $\ell \ell \ell$ decay signatures. Three events were found in $0.3 \mathrm{fb}^{-1}$ of data. An upper limit of the $\sigma_{p \bar{p} \rightarrow W Z / Z Z+X}$ at the $95 \%$ confidence level (CL) limit at $15.2 \mathrm{pb}[18]$.

This dissertation describes the cross section measurement of $W Z$ production using 1 $\mathrm{fb}^{-1}$ of $\mathrm{D} \varnothing$ data. The goal is to establish the $W Z$ signal at the Tevatron with at least $3 \sigma$ significance. 


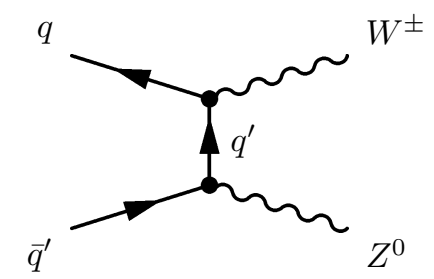

(a) t-Channel Feyman Diagram

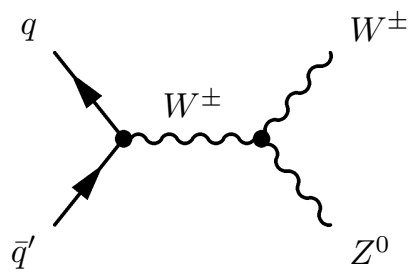

(b) s-Channel Feynman Diagram

Figure 1.1: The $t$ - (a) and $s$ - (b) channel tree level Feynman diagrams for $W Z$ production at the Tevatron. The $s$ channel contains that $W W Z$ vertex that allows us to directly measure the coupling strengths of the massive vector bosons to each other. If an anomalous signal is seen then there must be contributing interactions to the $W Z$ final state that are not accounted for in the SM. 


\section{CHAPTER 2}

\section{The Standard Model and the Physics of $W Z$ Production}

\subsection{The Standard Model and Particle Physics}

Over the last several decades the field of particle physics has developed through the great efforts of experimentalists and theorists. The goal of particle physics is to develop and test models of the fundamental constituents of matter and the forces that act between them. One such model has emerged which has been very successful at explaining experimental data and making predictions for the existence of new particles. This model has been referred to as the Standard Model (SM) of particle physics. The SM is a gauge theory that is constructed from the principles of gauge invariance and the Higgs mechanism [19].

In the SM all matter is made up of point-like particles called quarks and leptons. For each particle there is an anti-particle with the same mass but opposite electric charge. The forces between particles are mediated by the exchange of bosons. Three of the four fundamental forces of nature are described by the model: electromagnetism, the weak force and the strong force. The fourth force, gravity, is many orders of magnitude weaker than the others at the distance and energy scales available in the laboratory and is not included in the SM.

Leptons include the familiar electron and its heavier, unstable analogs the muon $(\mu)$ and tau $(\tau)$, all of which carry charge -1 . For each lepton there exists a corresponding 
neutral particle called a neutrino which has negligible mass.

There are six types of quarks: up $(u)$, charm $(c)$ and top $(t)$, and down $(d)$, strange $(s)$ and bottom $(b)$. Quarks posses fractional electric charge, $+\frac{2}{3}$ for up, strange, and top, and $-\frac{1}{3}$ for down, charm, and bottom. Quarks only exists as observables in the lab in pairs, known as mesons, or triplets, known as baryons, giving the composite particles an integer, $( \pm n$, or 0 where $n=1,2,3 \ldots)$ of electric charge. Quarks also carry color charge, which is associated with the strong force.

Quarks are the constituents of hadrons. Hadrons include the familiar proton and neutron and other unstable particles such as the pion. The proton is composed of two up quarks and a down quark which are bound together by the strong force. In proton antiproton, $p \bar{p}$, collisions quarks may be produced. Since quarks carry color charge they therefore undergo a process known as hadronization which creates colorless hadrons observed as hadronic jets.

Leptons and quarks are collectively called fermions. They are so named because they carry odd $\frac{1}{2}$ integer intrinsic angular momentum (spin) and obey the Pauli exclusion principle of Fermi-Dirac statistics.

Gauge bosons are spin-1 particles which are the carriers of the electromagnetic, weak and strong forces. They mediate the interactions between quarks and leptons. The massless photon carries the electromagnetic force over infinite distances. The gluons transmit the strong force over a range of order $1 \mathrm{fm}$ and the massive $W^{ \pm}$and $Z^{0}$ bosons transmit the weak force over much shorter distances.

In the SM the electromagnetic and weak interactions are unified into the electroweak force. The focus of this thesis is a test of the predictions of the electroweak interactions. The electroweak sector of the SM is discussed in the next section as a basis for further discussion. 


\section{$2.2 \quad$ Electroweak Interactions}

The $S U(2)_{L} \times U(1)_{Y}$ symmetry group is the basis of the SM of electroweak interactions [19],[20]. Four gauge fields are introduced: $W_{\mu}^{i}(i=1,2,3)$ for $S U(2)_{L}$ and $B_{\mu}$ for $U(1)_{Y}$. The fermions are written as left- and right-handed fields, which interact with these gauge fields. The left-handed fermion fields are written as isospin doublets

$$
\left(\begin{array}{c}
\nu_{L} \\
\ell_{L}
\end{array}\right)
$$

that transform under the $j=\frac{1}{2}$ representation of $S U(2)$. The right-handed fields are isospin singlets, $\ell_{R}$, that transform under the $j=0$ (trivial) representation of $S U(2)$.

The non-Abelian $S U(2)$ group is associated with weak-isospin $(I)$. The Abelian group $U(1)_{Y}$ is associated with the weak hypercharge, $Y$. The weak hyper-charge is related to the electric charge $(Q)$ and the weak-isospin by the Gell-Mann-Nishijima relation: $Q=I_{3}+Y / 2\left(I_{3}\right.$ is the third component of $\left.I\right)$.

To give the gauge bosons mass, an isospin doublet of complex scalar Higgs fields $\phi$ is introduced with a potential function which results in a non-zero vacuum expectation value for $\phi$. This results in the spontaneous breaking of the local $S U(2)$ gauge symmetry generating masses for the gauge bosons. Of the four fields only one corresponds to a physical particle, the Higgs boson [21]. The mass of the Higgs boson is a free parameter in the SM. The Higgs boson has not been experimentally observed to date.

The electroweak bosons are combinations of the $W_{\mu}^{i}$ and $B_{\mu}$ fields. Linear combinations of the $W_{\mu}^{1}$ and $W_{\mu}^{2}$ fields are identified as the $W_{\mu}^{ \pm}$fields. Linear combinations of the $W_{\mu}^{3}$ and $B_{\mu}$ fields are identified as the $Z$ field and the photon field, $A$.

A direct consequence of the Standard Model is the occurrence of gauge boson selfcouplings. This arises due to the non-Abelian character of the $S U(2)$ group. There are 
two types of interactions, the trilinear gauge couplings $(W W \gamma, W W Z)$ as illustrated in Figure 2.1, and quadralinear gauge couplings which are outside the scope of this thesis.

It is instructive at this point to review how this consequence arises. Development of non-Abelian gauge theories was motivated by interest in theories explaining isospin structures. Of particular interest to this thesis is the development of theories dealing with weak isospin. The challenge arises as one attempts to make a locally gauge invariant $\mathrm{SU}(2)$ field out of a globally invariant $\mathrm{SU}(2)$ free field theory. This means that when making infinitesimal transformations we require that the physics described by the theory before and after the transformation remain unchanged. This is local gauge invariance. The formulation proceeds by finding the proper gauge transformation for a particle field as

$$
\psi(x) \rightarrow \psi^{\prime}(x)=G(x) \psi(x)
$$

where $G(x) \equiv \exp \left(\frac{i}{2} \tau^{a} \cdot \alpha^{a}(x)\right)$ with $\tau^{a}$ being the Pauli isospin matrices, $\alpha^{a}(x)$ is an arbitrary position dependent (three vector) parameter of the transformation, and $a$ is the isospin index that runs from 1 to 3 . Then the gradient of the field transforms as

$$
\partial_{\mu} \psi \rightarrow G\left(\partial_{\mu} \psi\right)+\left(\partial_{\mu} G\right) \psi
$$

When applying the above gauge transformation to a free-particle Lagrangian,

$$
\mathcal{L}_{0}=\bar{\psi}(x)\left(i \gamma^{\mu} \partial_{\mu}-m\right) \psi(x)
$$

the gauge invariance is broken. The gauge invariance of the theory is maintained by introducing gauge fields to the gauge-covariant derivative

$$
\mathcal{D}_{\mu} \equiv I \partial_{\mu}+i g B_{\mu}
$$

where $I$ is as $2 \times 2$ unitary matrix and $g$ is a coupling constant. $B_{\mu}$ is a $2 \times 2$ matrix 
defined as

$$
B_{\mu}=\frac{1}{2} \tau^{a} b_{\mu}^{a}
$$

Where the three gauge fields are $b_{\mu}^{a}=\left(b_{1}, b_{2}, b_{3}\right)$. The gauge-covariant derivative must transform as the field, $\mathcal{D}_{\mu} \psi \rightarrow \mathcal{D}_{\mu}^{\prime} \psi^{\prime}=G\left(\mathcal{D}_{\mu} \psi\right)$. From this requirement it follows that the infinitesimal gauge fields transform as

$$
b_{\mu}^{\prime l}=b_{\mu}^{l}-\varepsilon_{j k l} \alpha^{j} b^{k}-\frac{1}{g} \partial_{\mu} \alpha^{l} .
$$

At this point we may construct an interaction Lagrangian that couples the isovector gauge fields to the conserved isospin current of the fields.

$$
\mathcal{L}=\bar{\psi}\left(i \gamma^{\mu} \mathcal{D}_{\mu}-m\right) \psi=\mathcal{L}_{0}-\frac{g}{2} b_{\mu} \cdot \bar{\psi} \gamma^{\mu} \tau \psi
$$

The last term in the lagrangian describes the interaction between the gauge field and the particles. This is a natural consequence of introducing the gauge-covariant derivative. To complete the Lagrangian a gauge invariant kinetic term for the gauge fields must be added to the Lagrangian:

$$
\mathcal{L}_{\text {gauge }}=-\frac{1}{2} \operatorname{tr}\left(F_{\mu \nu} F^{\mu \nu}\right)
$$

where the field strength tensor takes the form

$$
F_{\mu \nu}=\partial_{\nu} B_{\mu}-\partial_{\mu} B_{\nu}+i g\left[B_{\nu}, B_{\mu}\right]
$$

The complete Lagrangian is then

$$
\mathcal{L}=\bar{\psi}\left(i \gamma^{\mu} \mathcal{D}_{\mu}-m\right) \psi-\frac{1}{2} \operatorname{tr} F_{\mu \nu} F^{\mu \nu}
$$

It is a natural consequence of the non-Abelian characteristics of this field strength tensor that gives rise to the gauge field self interactions. In particular the nonlinear term in the $F_{\mu \nu}$ will generate the trilinear and quadralinear terms of the gauge bosons. 
We now apply this formalism to the electroweak isospin where Equation 2.2 is the left handed weak isospin vector. The gauge field term of the SM $S U(2)_{L} \times U(1)_{Y}$ Lagrangian is given by

$$
\mathcal{L}_{\text {gauge }}=-\frac{1}{4} F_{\mu \nu}^{l} F^{l \mu \nu}-\frac{1}{4} f_{\mu \nu} f^{\mu \nu}
$$

where the $S U(2)_{L}$ field strength tensors are

$$
F_{\mu \nu}^{l}=\partial_{\nu} b_{\mu}^{l}-\partial_{\mu} b_{\nu}^{l}+g \varepsilon_{j k l} b_{\mu}^{j} b_{\nu}^{k}
$$

and the $U(1)_{Y}$ field tensor is

$$
f_{\mu \nu}=\partial_{\nu} \mathcal{A}_{\mu}-\partial_{\mu} \mathcal{A}_{\nu}
$$

This Lagrangian leads to the prediction of the particular interactions seen in Figure 2.1.

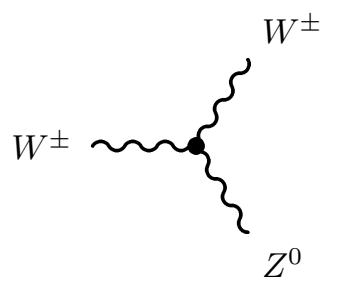

(a) $W W Z$ Vertex

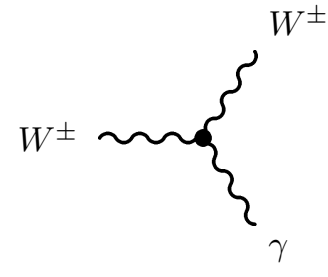

(b) $W W \gamma$ Vertex

Figure 2.1: Feynman diagrams of the trilinear gauge boson couplings allowed by the Standard Model. The $W W Z$ vertex is shown in (a) while the $W W \gamma$ vertex is shown in (b).

\section{3 $W Z$ Production in the Standard Model}

The focus of this study is the interaction between the $W$ and $Z$ gauge bosons, the most experimentally accessible of which are the trilinear gauge couplings. The Lagrangian $\mathcal{L}$ describing this portion of the electroweak sector, which is a derivation of Equation 2.2 is given by

$$
\mathcal{L}=-i e\left(W_{\mu}^{-} W_{\nu}^{+} A^{\mu \nu}+W_{\mu}^{+} W^{-\mu \nu} A_{\nu}-W^{i \mu \nu} W_{\mu}^{-} A_{\nu}\right)
$$




$$
-i e \cot \theta_{W}\left(W_{\mu}^{-} W_{\nu}^{+} Z^{\mu \nu}+W_{\nu}^{+} W^{-\mu \nu} Z_{\nu}-W^{+\mu \nu} W_{\mu}^{-} Z_{\mu}\right),
$$

where $\theta_{W}$ is the weak mixing angle, $W^{ \pm \mu}$ is the $W^{ \pm}$field, $Z^{\mu}$ is the $Z$ field, and $A^{\mu}$ is the photon field, and $W^{\mu \nu} \equiv \partial^{\mu} W^{\nu}-\partial^{\nu} W^{\mu}, A^{\mu \nu} \equiv \partial^{\mu} A^{\nu}-\partial^{\nu} A^{\mu}$ and $Z^{\mu \nu} \equiv \partial^{\mu} Z^{\nu}-$

$\partial^{\nu} Z^{\mu}$. These terms, which specify the $W W \gamma$ and $W W Z$ vertices respectively, arise due to non-Abelian gauge structure of the electroweak theory. When combined with fermion couplings to bosons, these terms completely describe $W Z$ production at the tree level in the Standard Model.

\subsection{1 $W Z$ Production Mechanisms}

There are three Feynman diagrams that describe tree level $W Z$ production at a hadron collider in the Standard Model, as shown in Figure 2.2. The first two diagrams represent $t$ - and $u$-channel $W Z$ production and are fully described by the couplings of the fermions to the $W$ and $Z$ bosons. These couplings have been measured with high precision for the production of single $W$ and $Z$ bosons [3],[4], [5],[6]. The third diagram shows $s$-channel $W Z$ production that involves the coupling of the $W$ and $Z$ bosons, i.e. the $W W Z$ coupling. Therefore an experimental study of $W Z$ production enables the measurement of the $W W Z$ coupling.

\subsubsection{Standard Model Predictions for $W Z$ Production}

Once the tree level Feynman diagrams for $W Z$ production are known, it is possible to predict observables such as the cross section. The calculation of the $W Z$ production cross section leads to an insight into the structure of the SM. If only the $t$ - and $u$ channel diagrams were used to calculate the total cross section, the result would be a linear rise of the cross section with increasing $\sqrt{\hat{s}}$ (the parton center of mass energy). 


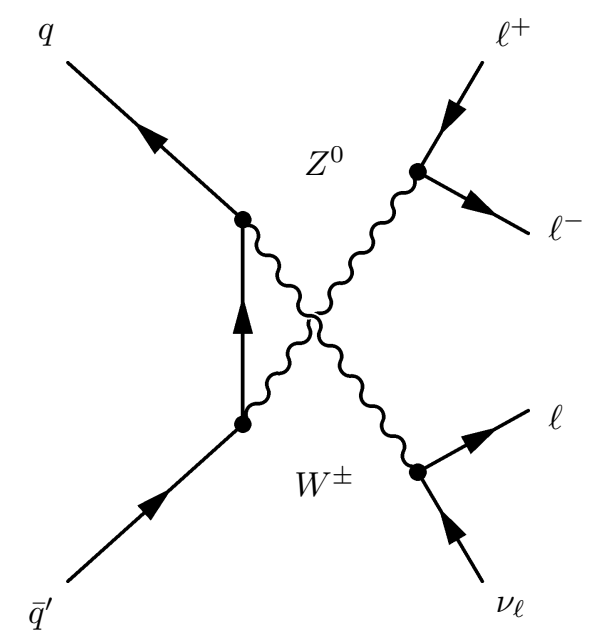

(a) u-Channel Production Mechanism

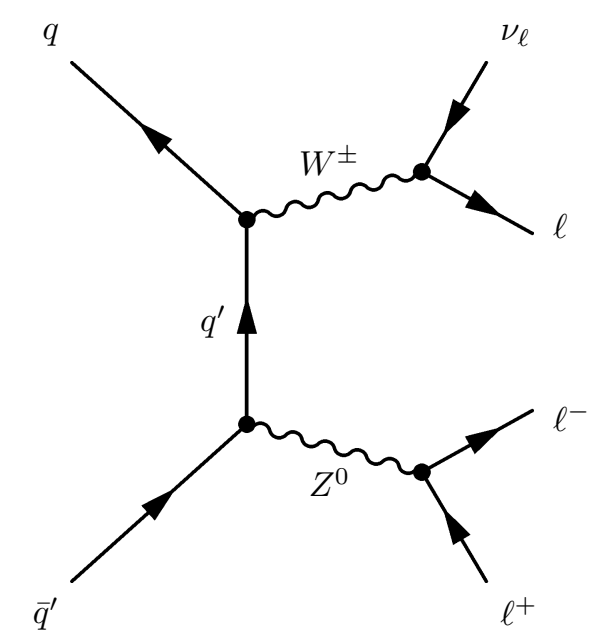

(b) t-Channel Production Mechanism

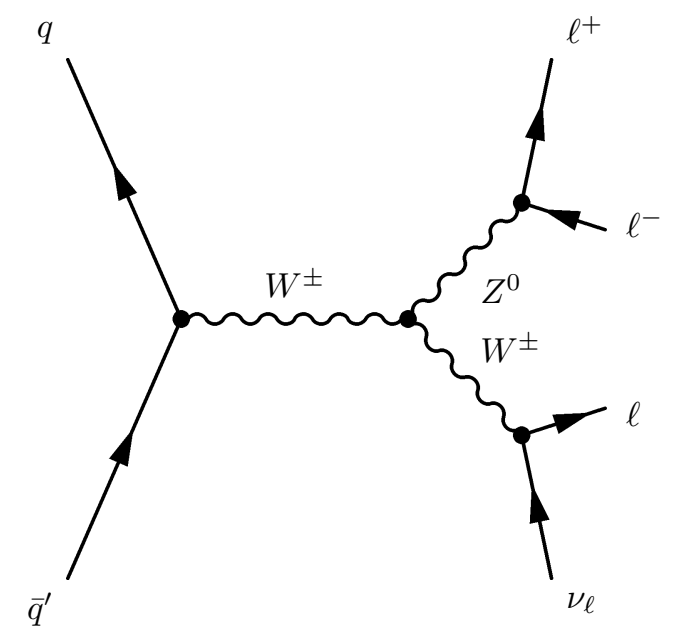

(c) s-Channel Production Mechanism

Figure 2.2: Standard Model Feynman Diagrams for tree level $W Z$ production with subsequent decay into leptons: (a) $u$-channel, (b) $t$-channel, (c) $s$-channel. 
This implies that for sufficiently large energies, partial wave unitarity will be violated, i.e. the sum of the probabilities of the partial waves will be greater than one. By including the $s$-channel diagrams, which involve the boson-boson couplings, the cross terms that result from squaring all the summed amplitudes provide the "delicate" gauge cancellations which are required to restore unitarity. By construction, the SM provides the gauge boson self-interaction terms that restore the physical consistency of the model. These terms are unnecessary to describe many weak current interactions, such as $\beta$ decay. This cancellation will have important consequences in the search for deviations from the SM predicted values for the boson-boson couplings.

A numerical result for the $W Z$ production cross section cannot be produced analytically because of the composite nature of the proton and antiproton. The parton subprocess cross section can be computed analytically, but this must be summed over all possible pairs of participating partons in the proton and antiproton and additionally integrated over the parton momentum distributions. A Monte Carlo approach can be used to solve this problem. Event generators such as PYTHIA[22] can be used to fully model Standard Model $W Z$ production and can be used to produce a numerical results for the cross section. The cross section for the PYTHIA generator is corrected with a next to leading order (NLO) calculations where the $k$ factor, at the energy of the Tevatron, is approximately 1.3. The NLO calculations takes into account the initial and final state gluon radiations and one-loop corrections. The $k$-factor is defined as the ratio of the NLO MC predictions to the LO MC predictions, $k \equiv \frac{\sigma_{\mathrm{NLO}}}{\sigma_{\mathrm{LO}}}$.

\subsubsection{Experimental Signature of $W Z$ Production}

$W Z$ production can occur in three distinct channels: those in which both bosons decay hadronically, those in which one decays hadronically and the other leptonically, and those 
in which both decay leptonically.

The purely hadronic final state has one advantage. It has a significantly larger branching fraction than all leptonic decays. However the disadvantages far outweigh this advantage. First, it is nearly impossible to determine from which boson a reconstructed hadronic jet came. This is due to the finite energy resolution of hadronic calorimeters and to the difficulty of the charge sign determination of jets. Further, the limited energy resolution of hadronic calorimeters makes distinguishing $W$ 's from $Z$ 's difficult at best. $W W$ and $W Z$ production are therefore indistinguishable in this channel. Finally this channel suffers from a large background due to continuum multijet production as well as the production of single $W$ or $Z$ bosons in association with jets.

The semi-leptonic decay modes have the next largest branching fractions, $15 \%$ for the $l \nu j j$ final state and $4.5 \%$ for the $l l j j$ final state, where $l$ is an electron or muon and $j$ is a hadron jet. This channel suffers from large QCD backgrounds from both multijet production and $W$ production in association with jets, which is indistinguishable from Standard Model $W Z$ production. As in the fully hadronic channel, it is impossible to distinguish $W Z$ production from $W W$ production in the $l \nu j j$ channel. The $l l j j$ final state has the advantage of being identified only with $W Z$ production. However, this final state is dominated by backgrounds from $Z$ production in association with jets. The main advantages of this channel are the relatively large branching fraction and the ability to unambiguously reconstruct the momentum of each boson. A cross section measurement in the semi-leptonic channel is insensitive due to the inability to distinguish signal from background.

The purely leptonic final state has the smallest branching fraction of all, $1.5 \%$ when both electrons and muons are counted ( $\tau$ 's are excluded due to the difficulty in identifying them efficiently). The one drawback of this channel is the relatively small branching frac- 
tion. The main advantage of this channel is its unique signature, three charged leptons with high transverse momentum $\left(p_{T}\right)$, and large missing transverse energy $\left(\mathbb{E}_{T}\right)$. This signature is unique amongst diboson final states and is virtually background free. No physics processes produce a significant background. The only backgrounds are instrumental backgrounds, which arise from the misidentification of a jet as a lepton.

As a result of these factors, $W Z$ production in the purely leptonic decay mode provides a sensitive measure of the cross section and a direct measure of the $W W Z$ vertex. The search for $W Z$ production in the purely leptonic final state is the subject of this thesis.

\subsection{Summary of the Theoretical Prediction}

The most recent update of the predicted cross section for the Tevatron Run II comes from the application of the PDF set of CTEQ6M with the MCFM generator. The predicted cross section from this analysis [23] is $3.68 \pm 0.22$ (scale) \pm 0.12 (PDF) pb for real $W Z$ production. The scale uncertainty is evaluated by varying the renormalization and factorization scale between $(M(W)+M(Z)) / 4<$ scale $<(M(W)+M(Z))$, where $M(W)$ and $M(Z)$ are the masses of the produced $W$ and $Z$ bosons. The PDF uncertainties are evaluated with error PDF sets at LO. 


\section{CHAPTER 3}

\section{Experimental Apparatus}

The Fermi National Accelerator Laboratory (FNAL. Fermilab)[24] operates the world's highest energy proton-antiproton collider, the Tevatron. In this chapter, the chain of accelerators that achieve a center-of-mass collision energy of $1.96 \mathrm{TeV}$ is described. An overview of the $\mathrm{D} \varnothing$ detector, which is built around one of the interaction regions where proton and anitprotons collide, is given.

The data used in the analysis were recorded with the $\mathrm{D} \emptyset$ detector during the data taking period known as Run IIA, which officially began in March 2001 and ended October 2006.

\subsection{The Accelerators}

The Fermilab particle accelerators [12] consist of seven separate devices, as shown in Figure 3.1: the Cockcroft-Walton pre-accelerator, the Linac linear accelerator, the Booster synchrotron, the Main Injector, the Antiproton Source, the antiproton Recycler, and the Tevatron. The Antiproton Source has three components: an antiproton target, a Debuncher, and an Accumulator. An overview of each device is given the following sections. 


\section{FERMILAB'S ACCELERATOR CHAIN}

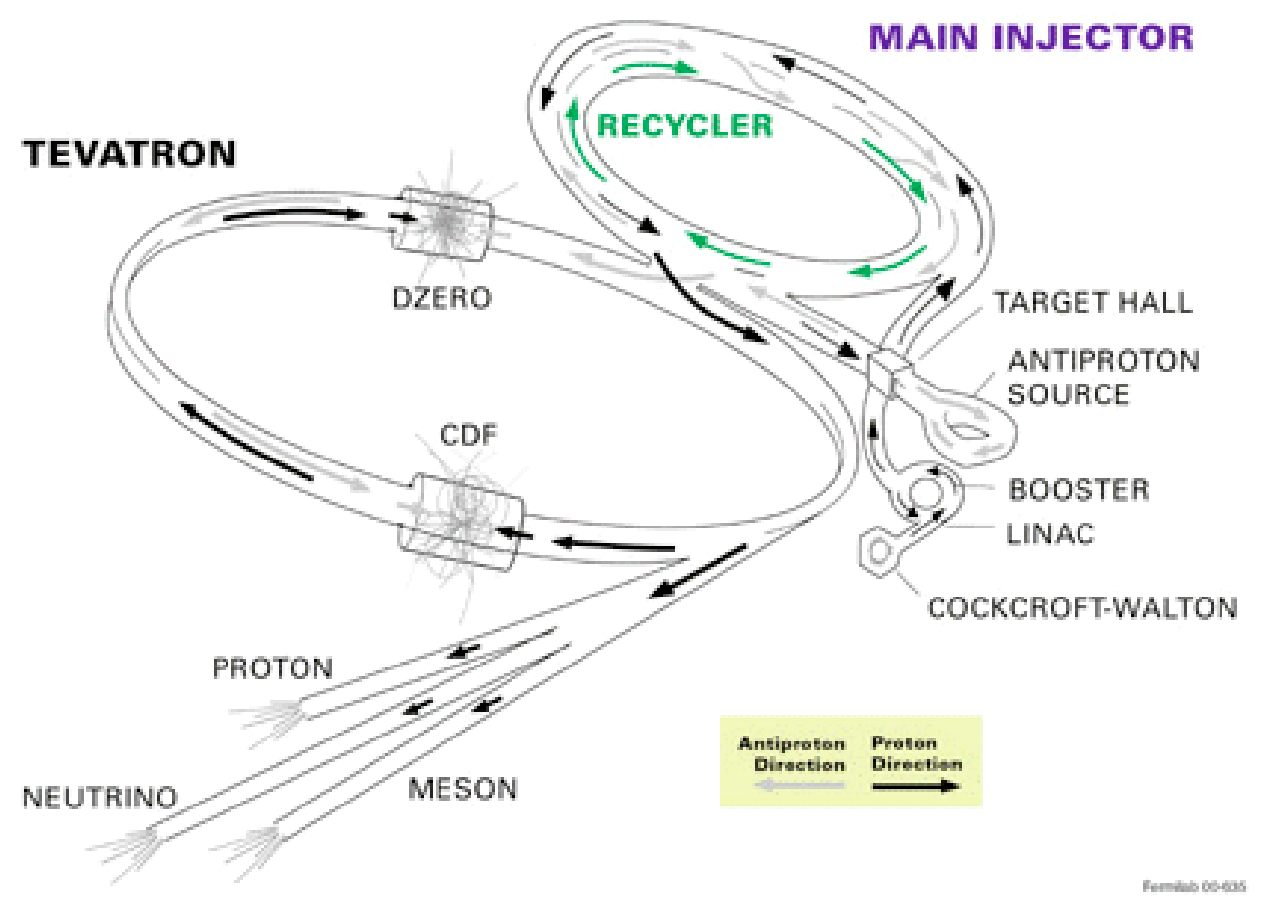

Figure 3.1: The schematic view of the accelerator at Fermilab. The Recycler is housed above the Main Injector. The Debuncher and Accumulator are in the Antiproton Source ring. 


\subsubsection{The Cockroft Walton Pre-Accelerator}

The Cockroft-Walton pre-accelerator starts by turning hydrogen gas $\left(\mathrm{H}_{2}\right)$ into hydrogen ions $\left(\mathrm{H}^{-}\right)$. The hydrogen gas is pulsed at $15 \mathrm{~Hz}$ with a pulse duration of $80 \mu \mathrm{s}$. The $\mathrm{H}^{-}$ ions are produced by a Magnetron Surface Plasma Sourse (MSPS). The MSPS (Figure 3.2) is an oval shaped cathode surrounded by an anode with a uniform magnetic field passing through it. Cesium vapor coats the surface of the cathode allowing electrons to be pulled away from the cathode. The electric field strips the electrons from the hydrogen gas while the magnetic field causes the charged particles to move in a helical paths. The $\mathrm{H}^{+}$ions accumulate on the cathode. Eventually, a charged particle will remove the $\mathrm{H}^{+}$ ion from the cathode. Some of these $\mathrm{H}^{+}$ions release two electrons from the cesium coated surface. The $\mathrm{H}^{-}$ions migrate out of the enclosing anode and are accelerated by a $18 \mathrm{keV}$ extractor plate. The accelerated particles are passed though a bending magnet which extracts the $\mathrm{H}^{-}$ions. The $18 \mathrm{keV}$ hydrogen ion beam then enters the Cockroft-Walton, which is seen in Figure 3.3. The Cockroft-Walton uses static electric fields to accelerate the ions. The electric fields are generated by charging cpacitors in parallel and discharging to $750 \mathrm{keV}$. Before entering the next accelerator, the Linac, the $80 \mu s$ continuous beam is subdivided into a 201.24 MHz "bunched" beam using an electrostatic chopper and a single gap radio frequency $(\mathrm{RF})$ cavity.

\subsubsection{Linac}

The Linac is a linear accelerator broken into two sections: a series of $201.24 \mathrm{MHz} \mathrm{RF}$ accelerating cavities, (Figure 3.4), and a series of $804.96 \mathrm{MHz}$ accelerating cavities. As the beam passes through a series of RF cavities, the resonant cells become progressively longer. Each RF cavity has a resonant RF of 201.24 MHz or $804.96 \mathrm{MHz}$ and contains a drift tube. The beam stays together via alternating focusing and defocusing quadrupole 


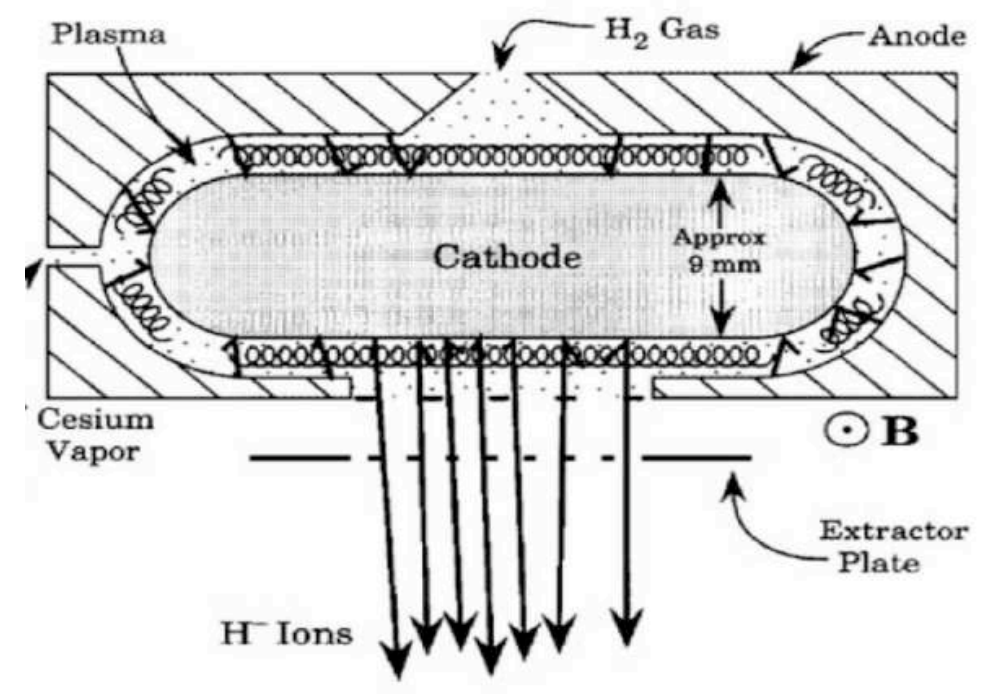

Figure 3.2: Hand drawing of the $\mathrm{H}^{-}$ion source or magnetron.

magnets embedded within the drift tubes, the $\mathrm{H}^{-}$ions are accelerated. The $\mathrm{H}^{-}$ions then leave the Linac at energies of $400 \mathrm{MeV}$ and are transfered to the Booster.

\subsubsection{Booster}

The Booster is the first synchrotron accelerator in the Fermilab accelerator complex. The Booster operates in three modes: fill, accelerate, and dump. During the fill mode, the $\mathrm{H}^{-}$ions pass through a $\mathrm{RF}$ debuncher which keeps the beam energy fixed at 400 $\mathrm{MeV}$ while reducing the momentum spread and removing the $804.96 \mathrm{MHz}$ RF structure. The incoming $\mathrm{H}^{-}$ions are deflected from their orbital paths during filling just before a straight section in the beam line. Two opposite polarity dipole magnets merge the $\mathrm{H}^{-}$ and the $\mathrm{H}^{+}$ions into a single beam. The combined beam is then passed through a carbon foil to strip the electrons away from the $\mathrm{H}^{-}$ions. The carbon foil causes some beam loss due to scattering. The resulting proton beam is deflected back to the proper orbital path. After accumulating $3 \times 10^{12}$ protons in the Booster, the fill process stops, and the magnets which deflected the protons from their orbit are turned off. The beam is then accelerated to $8 \mathrm{GeV}$ by increasing the magnetic fields to maintain the protons at 


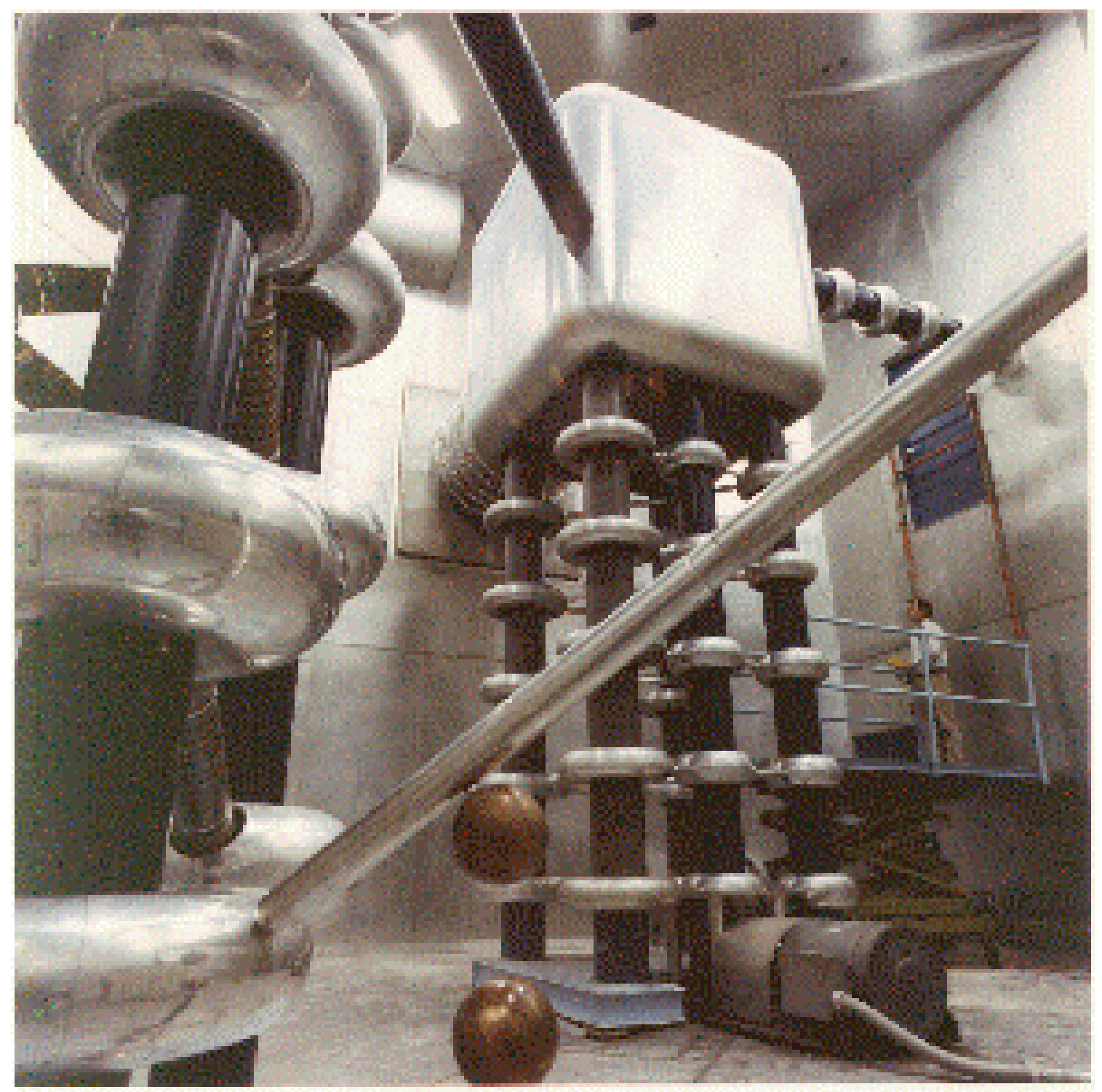

Figure 3.3: Photo of the Cockroft-Walton pre-accelerator.

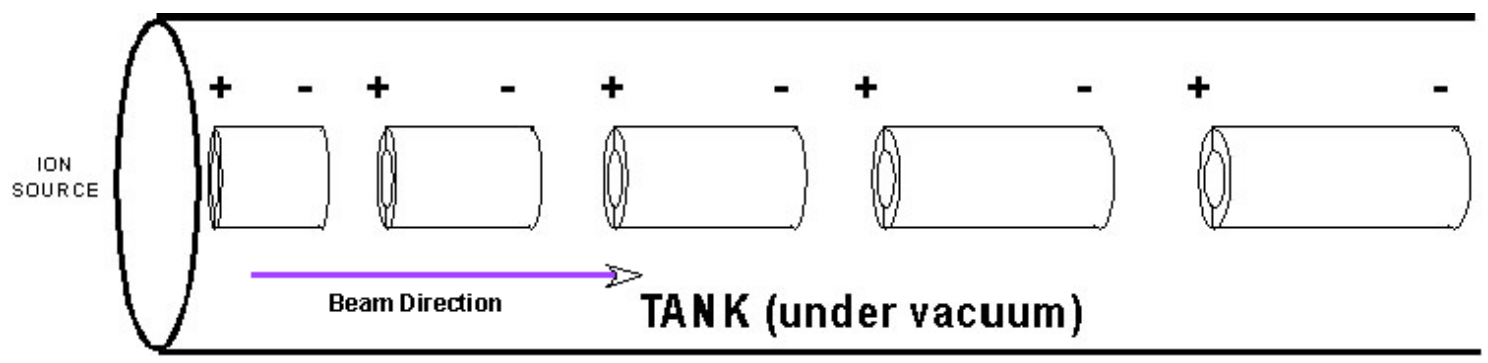

Figure 3.4: A drawing of the Linac RF cavity. 
the same radius, and increasing the RF. After accleration, the Booster switches to dump mode and bunched protons are sent to the Main Injector.

\subsubsection{Main Injector}

The Main Injector is the second synchrotron accelerator in the chain of accelerators. The Main Injector, receives protons from the Booster and perfoms the following tasks:

- acceleration of $8 \mathrm{GeV}$ protons from the Booster to $120 \mathrm{GeV}$ and delivery to the antiproton source,

- acceleration of $8 \mathrm{GeV}$ protons from the Booster to $120 \mathrm{GeV}$ and delivery to the fixed target lines,

- acceleration of $8 \mathrm{GeV}$ protons from the Booster to $150 \mathrm{GeV}$ and delivery to the Tevatron,

- acceleration of $8 \mathrm{GeV}$ antiprotons from the Accumulator to $150 \mathrm{GeV}$ and delivery to the Tevatron,

- acceleration of $8 \mathrm{GeV}$ antiprotons from the Recycler to $150 \mathrm{GeV}$ and delivery to the Tevatron.

\subsubsection{Antiproton Source}

The antiproton source has three components: the fixed target, the Debuncher, and the Accumulator. $120 \mathrm{GeV}$ protons from the Main Injector strike the nickel target (Figure 3.5 and Figure 3.6) and are collected using a lithium lens (Figure 3.7) and a dipole magnet for momentum selection. A drawing of the anitproton source is shown in Figure 3.7. The limited ability to capture $8 \mathrm{GeV}$ antiprotons inside a small acceptance area yields only 12-24 antiprotons per million protons which hit the nickel target. Since the antiproton generation efficiency is so low, it takes more than twelve hours to generate enough antipro-

tons to begin $\bar{p} p$ collisions. Using a process called stochastic cooling, the transverse and 
longitudinal dispersion of the $8 \mathrm{GeV}$ antiprotons is minimized in the Debuncher before the antiprotons are transferred to the Accumulator. The Accumulator stores antiprotons until enough antiprotons have been collected to either start high energy physics collisions or to transfer the antiprotons to the Recycler. The Accumulator uses transverse and longitudinal adjustment techniques to minimize the dispersion while more antiprotons are stored.

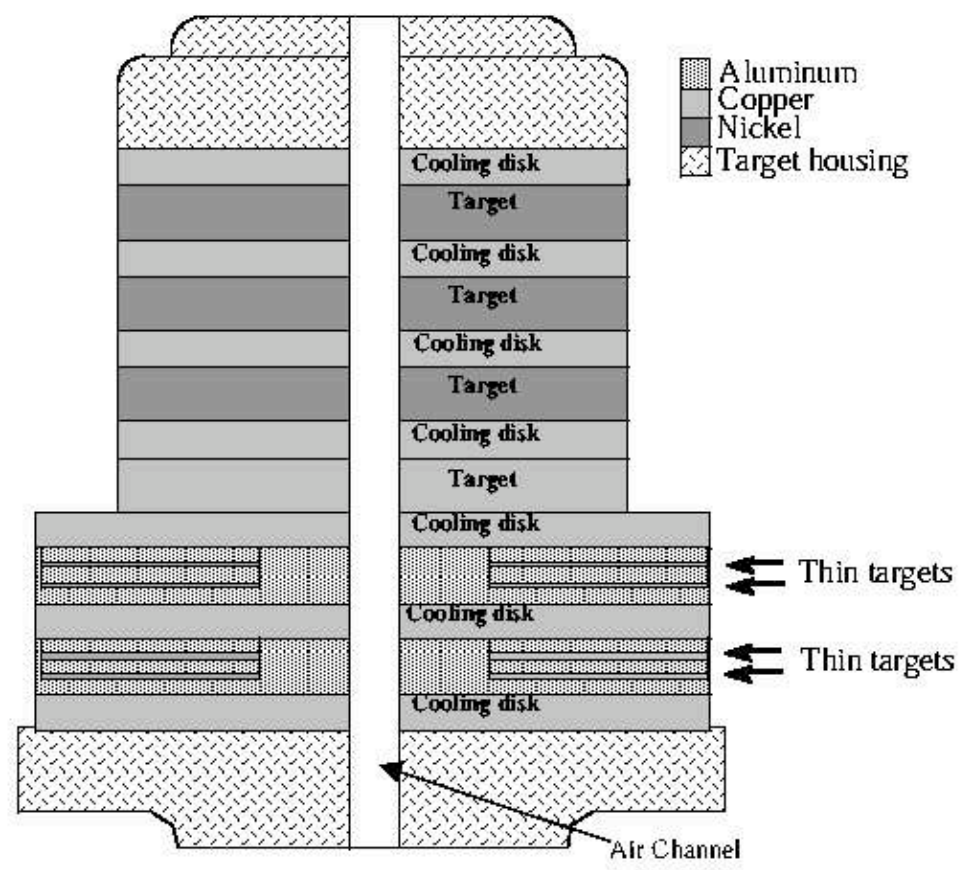

Figure 3.5: A schematic of the antiproton generating nickel target.

\subsubsection{Recycler}

The Recycler is an antiproton storage ring located above the Main Injector designed to operate in two ways: holding of $8 \mathrm{GeV}$ antiprotons from the Accumulator or storing of the remnants of a colliding beam. The efficiency of the Accumulator to accept more antiprotons from the Debuncher decreases as the number of antiprotons stored in the Accumulator increases. 


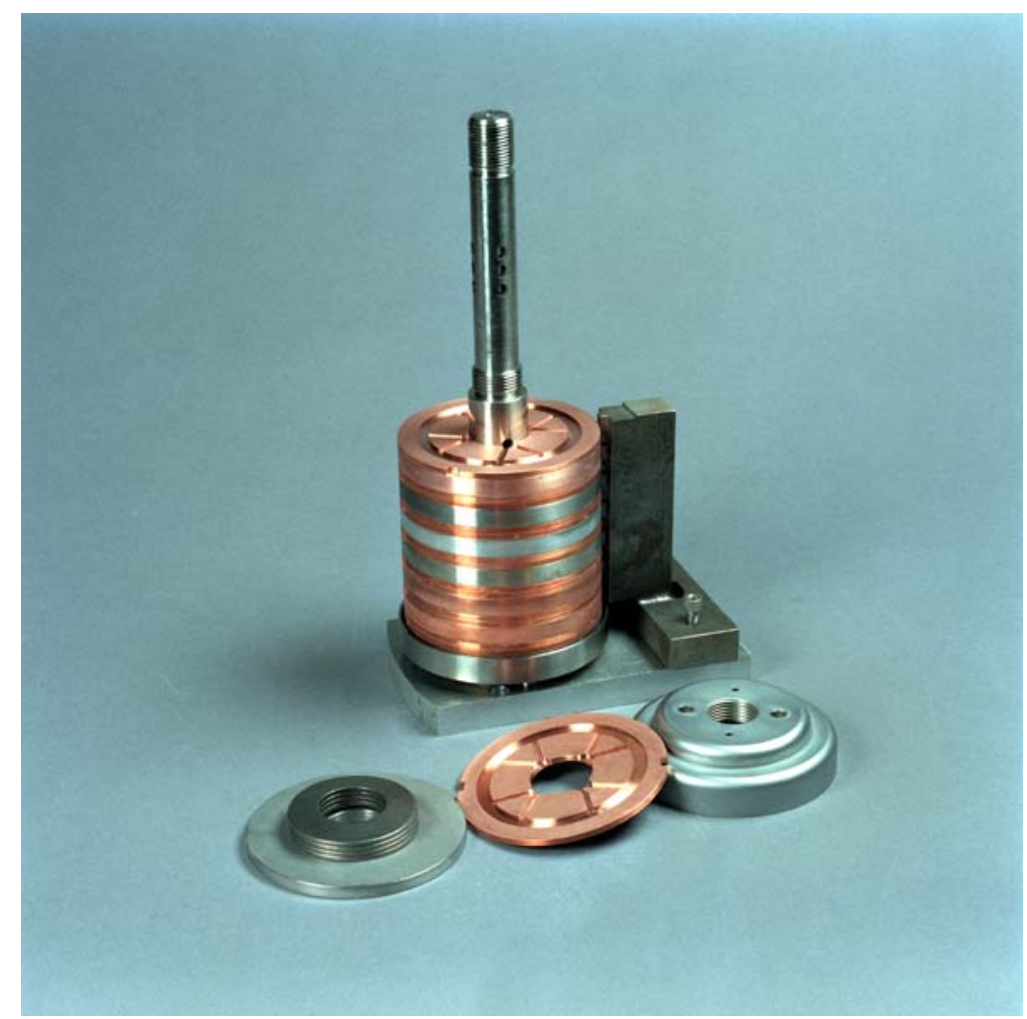

Figure 3.6: A photograph of the actual nickel target.

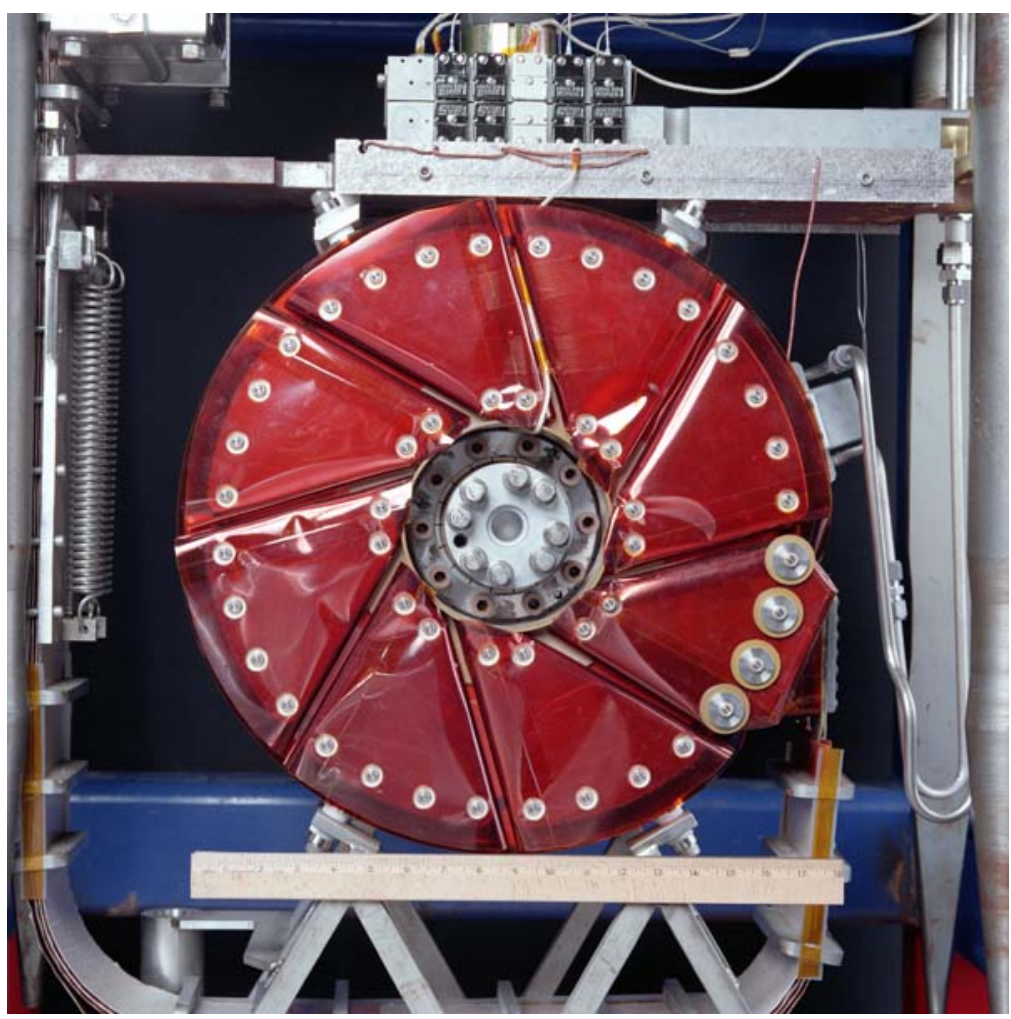

Figure 3.7: A photograph of the Lithium lens showing the beam's eye view. 


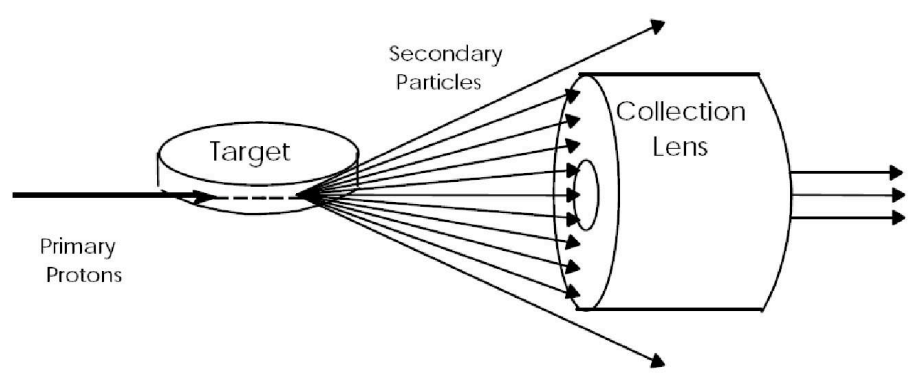

Figure 3.8: A diagram showing the nickel target and lithium lens.

\subsubsection{Tevatron}

The Tevatron is a synchrotron accelerator that uses superconducting magnets (up to $\sim 4 \mathrm{~T}$ ) to generate magnetic fields strong enough to allow the RF cavities to accelerate the protons and antiprotons to $980 \mathrm{GeV}$ within a $1 \mathrm{~km}$ radius circular ring.

The Main Injector sends 36 bunches of protons into the Tevatron, one bunch at a time. After all the protons have been loaded into the Tevatron, the Main Injector sends 36 bunches of antiprotons into the Tevatron, four bunches at a time. The two beams follow the same orbital paths, but in opposite directions and the beams do not collide since they are forced to travel in helical paths. The 36 bunches are separated into three super bunches separated by $2.64 \mu \mathrm{s}$. Within a super bunch, the twelve bunches are separated by 396 ns.

The two beams are magnetically squeezed and forced to pass through each other at two separate interaction points where the CDF and $\mathrm{D} \emptyset$ detectors are based. A store is defined as the period of time in which the two beams are colliding. A typical store lasts twenty-four hours or more based on the initial density per bunch of antiprotons in the accelerator. 


\subsection{The DØ Detector}

The D $\varnothing$ detector [25] was built to study high mass states and large $p_{T}$ phenomena. The $D \varnothing$ detector was built using a variety of particle detectors installed in layers as shown in Figure 3.9. The innermost layer includes the track finding detector for charged particles surrounded by a sampling calorimeter. The calorimeter is then surrounded by a muon spectrometer. The central tracking system starts with a silicon microstrip tracker, a central scintillating fiber tracker, and a solenoid producing an internal $2 \mathrm{~T}$ magnetic field. A preshower scintillating fiber detector is located between the solenoid magnet and the calorimeter. Planes of scintillating tiles, used for measuring luminosity, surround the beam pipe at two ends of the detector. The sampling calorimeter is segmented into three separate detectors (one central and two end detectors). The three calorimeter detectors have absorbers made of depleted uranium, copper, and stainless steel. Liquid argon is the active medium inside the calorimeters. The muon detector system has a $1.8 \mathrm{~T}$ toroid with scintillator counters, proportion drift tubes, and mini drift tubes.

\subsubsection{The DØ Coordinate System}

The D $\varnothing$ coordinate system uses a mix of cylindrical coordinates and spherical coordinates. There are three variables as shown in Figure $3.10(r, \phi$, and $\theta)$. The $z$ axis is taken along the beam pipe in the direction of the protons. $\phi$ and $\theta$ have the same meaning as in the spherical coordinate system, but $r$ is defined by the cylindrical coordinates system. $r, \phi$, and $\theta$ in terms of $x, y$, and $z$ are defined as follows:

$$
\begin{gathered}
r^{2}=x^{2}+y^{2}, \\
\phi=\arctan \left(\frac{y}{x}\right),
\end{gathered}
$$




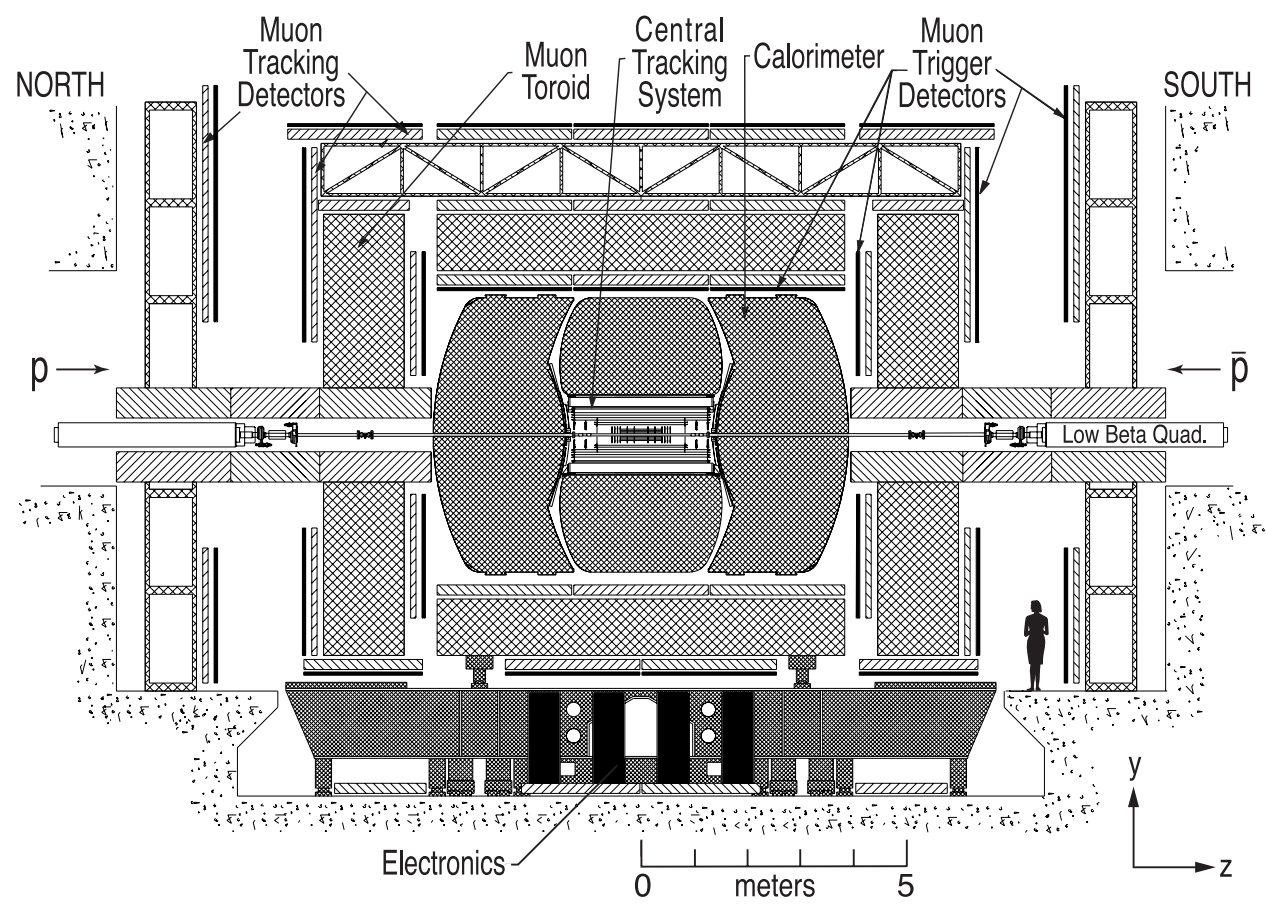

Figure 3.9: Side view of the $\mathrm{D} \varnothing$ detector.

$$
\theta=\arctan \left(\frac{r}{z}\right)
$$

Instead of using the scattering angle $\theta$, pseudorapidity, $\eta$, is used. This has the property that the number of particles produced in a given range of $\eta$ is invariant with respect to boosts along the $z$ direction when the mass of the particles is zero. If the mass of the particles is not equal to zero, then the proper angle to use is rapidity, $y$. Rapidity, pseudorapidity, and $\theta$ are defined as:

$$
\begin{gathered}
y=\frac{1}{2} \log \left(\frac{E+p_{z}}{E-p_{z}}\right), y=\eta \text { when } m=0 \text { or } \frac{m}{E} \rightarrow 0, \\
\eta=-\log \left(\tan \left(\frac{\theta}{2}\right)\right)=\cosh ^{-1}\left(\frac{1}{\sin (\theta)}\right), \\
\theta=2 \arctan \left(e^{-\eta}\right)=\arcsin \left(\frac{1}{\cosh (\eta)}\right) .
\end{gathered}
$$




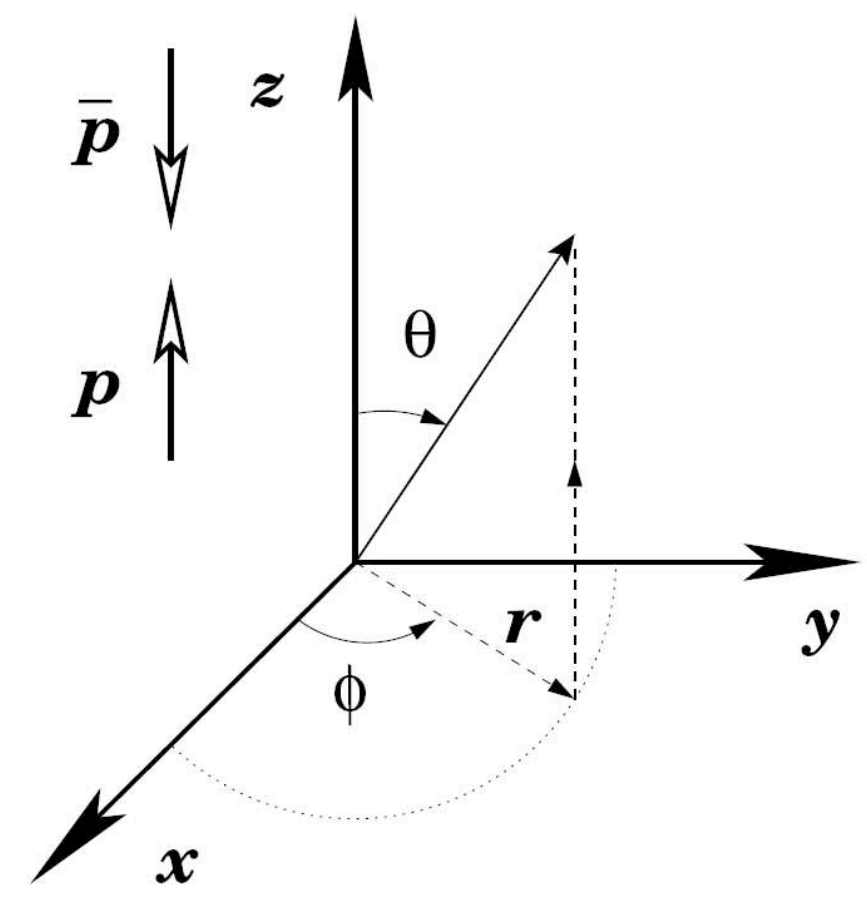

Figure 3.10: The DØ detector coordinate system.

The energy and momentum of a particle using rapidity is given by:

$$
\begin{aligned}
& E=m_{T} \cosh (y), \\
& p_{z}=m_{T} \sinh (y),
\end{aligned}
$$

where the transverse mass, $m_{T}$, is defined as:

$$
m_{T}^{2}=E_{T}^{2}-p_{x}^{2}-p_{y}^{2}
$$

The transverse momentum is the projection of the momentum vector to the $(x, y)$ plane:

$$
p_{T}=p \sin (\theta)=\frac{p}{\cosh (\eta)} .
$$


The limit of $p_{T} \rightarrow E_{T}$ as $m \rightarrow 0$ :

$$
E_{T}=E \sin (\theta)=\frac{E}{\cosh (\eta)} .
$$

Finally $p_{x}, p_{y}$, and $p_{z}$ are calculated using $p, \phi$, and $\eta$ via:

$$
\begin{gathered}
p_{x}=p_{T} \cos (\phi)=\frac{p}{\cosh (\eta)} \cos (\phi), \\
p_{y}=p_{T} \sin (\phi)=\frac{p}{\cosh (\eta)} \sin (\phi), \\
p_{z}=p \cos (\theta)=p \cos \left(\arcsin \left(\frac{1}{\cosh (\eta)}\right)\right) .
\end{gathered}
$$

\subsection{Luminosity Monitor}

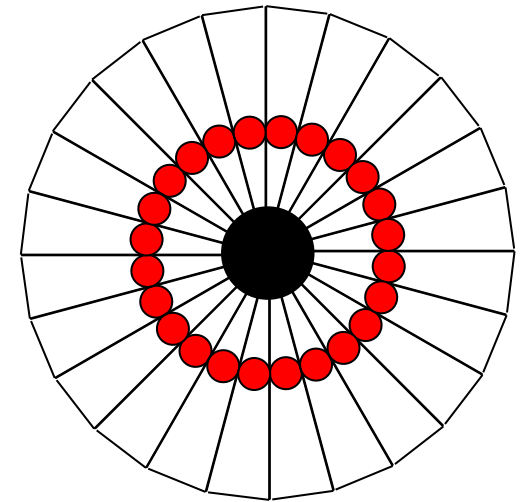

(a) $r-\phi$ view

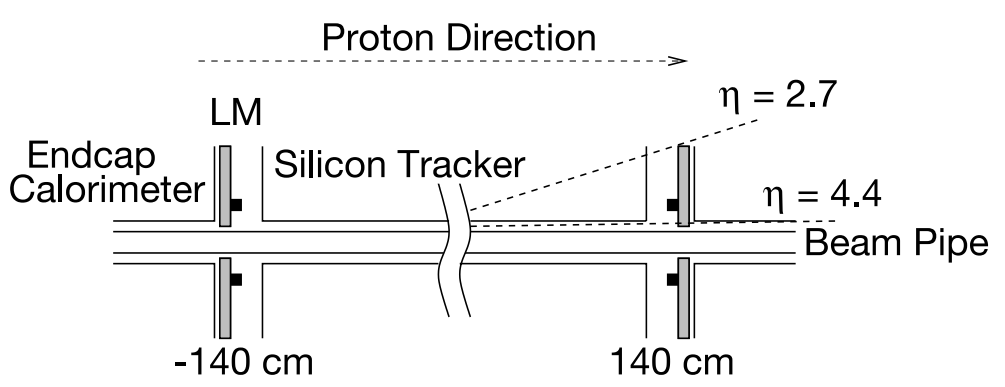

(b) $r-z$ view

Figure 3.11: A diagram of the $\mathrm{D} \emptyset$ luminosity monitors. The $r-\phi$ view is shown on the left, while the $r-z$ view of the two monitors is shown on the right. The photomultiplier tubes are represented by small circles on each tile.

The number of $\bar{p} p$ collisions over a given time and area is calculated using the luminosity detector [26]. The two luminosity detectors are located at $\pm 140 \mathrm{~cm}$ from the center of the $\mathrm{D} \varnothing$ detector along the $z$ direction. The luminosity detectors cover the $\eta$ range of 


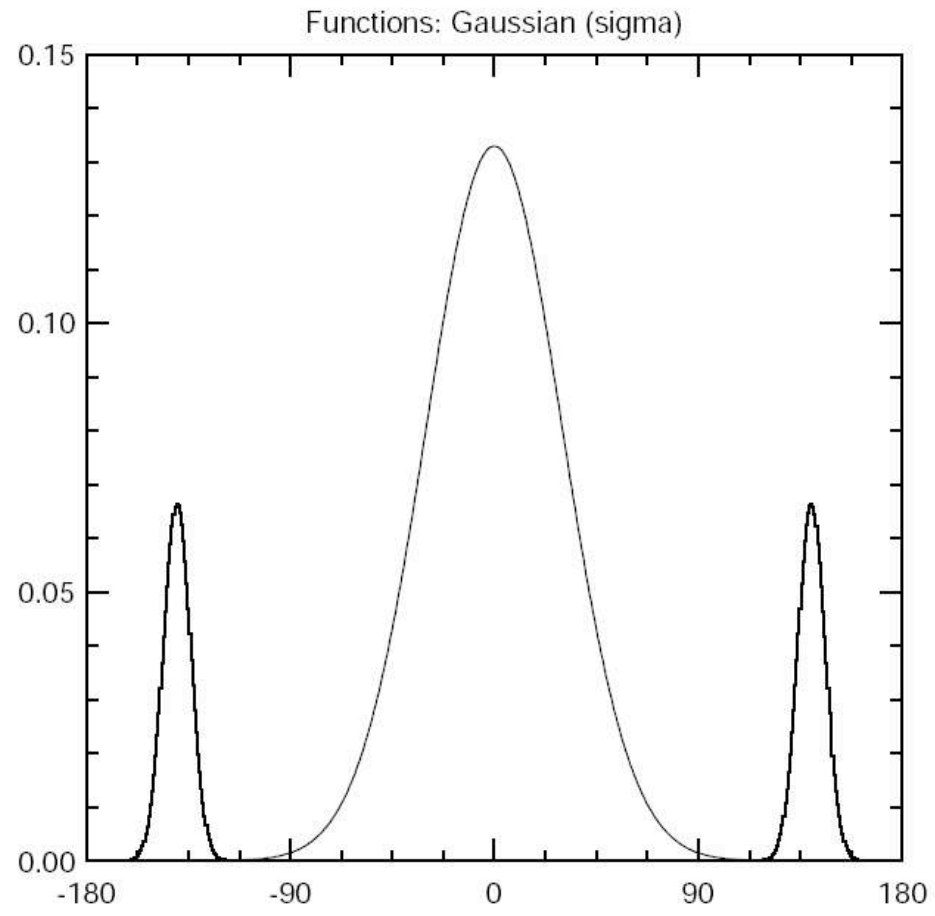

Figure 3.12: The $z$ vertex distribution from inelastic $\bar{p} p$ collisions.

$2.7<|\eta|<4.4$ in a $\phi$ ring (Figure 3.11). Each detector consists of twenty-four wedge shaped scintillating tiles that are $15 \mathrm{~cm}$ long and $1.6 \mathrm{~cm}$ thick. The scintillating tiles are read out by high gain photomultiplier tubes attached perpendicularly to each tile. Using the time of flight equation $z_{v}=\frac{c}{2}\left(t_{-}-t_{+}\right)$, where $t_{-}$and $t_{+}$are the times when the decay products hit the downstream and upstream luminosity monitors respectively, a quick location of $z$ position of the primary vertex is determined, as shown in Figure 3.12. Only events with $\left|z_{v}\right|<100 \mathrm{~cm}$ are used to count the number $\bar{p} p$ collisions. The instantaneous luminosity is a measure of the particle flux per unit area per unit time $\left(\mathrm{cm}^{-2} \mathrm{~s}^{-1}\right)$, and is defined as:

$$
L=\frac{f \bar{N}_{L M}}{\sigma_{L M}},
$$

where $f$ is the beam crossing frequency $\left(\frac{1}{396} \mathrm{~ns}^{-1}\right.$ or $\left.2.53 \mathrm{MHz}\right), \bar{N}_{L M}$ is the average number 
of $\bar{p} p$ inelastic collisions for one crossing, and $\sigma_{L M}$ is the effective cross section (taking into account the acceptance and efficiency of the luminosity monitor). The effective cross section is the measure of the interaction probability per unit flux ${ }^{1}$.

\section{B. Run II Integrated Luminosity 19 April 2002 - 29 August 2006}

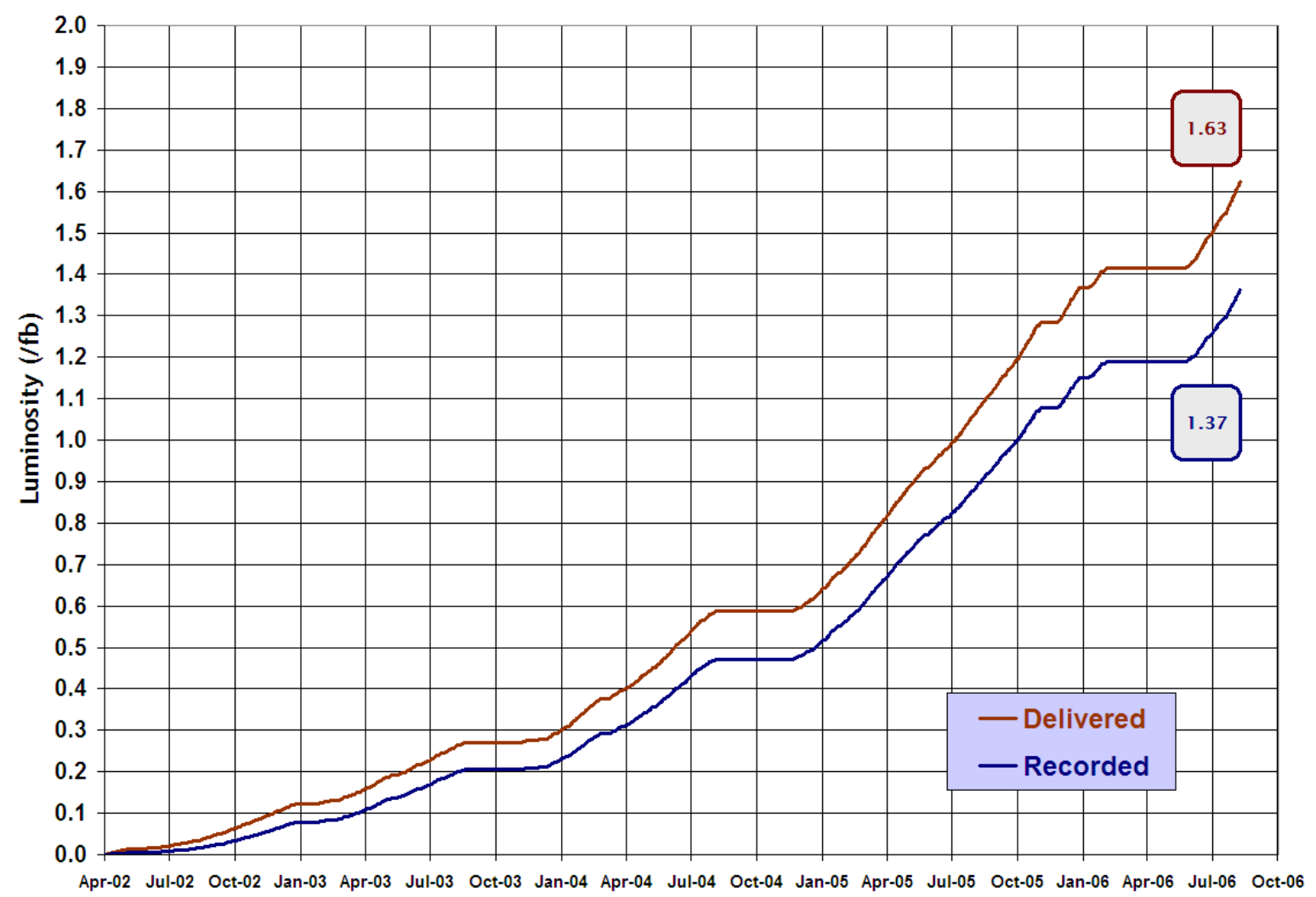

Figure 3.13: The total amount of luminosity delivered to and recorded by the D $\varnothing$ detector.

\subsubsection{The Central Tracking System}

The central tracking system (Figure 3.14) measures the sign of the charge, the position, and the momentum of charged particles transversing the following set of detectors:

- the 2 Tesla solenoid,

- the silicon microstrip tracker,

\footnotetext{
${ }^{1}$ Cross sections are usually expressed in barns, where 1 barn $=10^{-24} \mathrm{~cm}^{2}$. Integrated luminosity is expressed in $\mathrm{b}^{-1}$.
} 
- the central fiber tracker.

The central tracking system depends on the Lorentz force $(\vec{F}=q \vec{v} \otimes \vec{B})$ to bend the path of particles with charge $(q)$ based on the strength of the magnetic field $(\vec{B})$ and velocity $(\vec{v})$ of the particles. The solenoid generates a uniform $2 \mathrm{~T}$ magnetic field along the $z$ direction causing the charged particles to travel in helical paths characterized by the radius of curvature $r=\frac{p_{T}}{q B}$ and $p_{z}$. The charge of a particle is measured by the direction of the deflection. The momentum is measured by the curvature of the charged particle. The charged particles deposit small amounts of ionization energy into the silicon and scintillating fibers. A collection of small energy deposits (hits) are used to reconstruct the path or track of the particles.

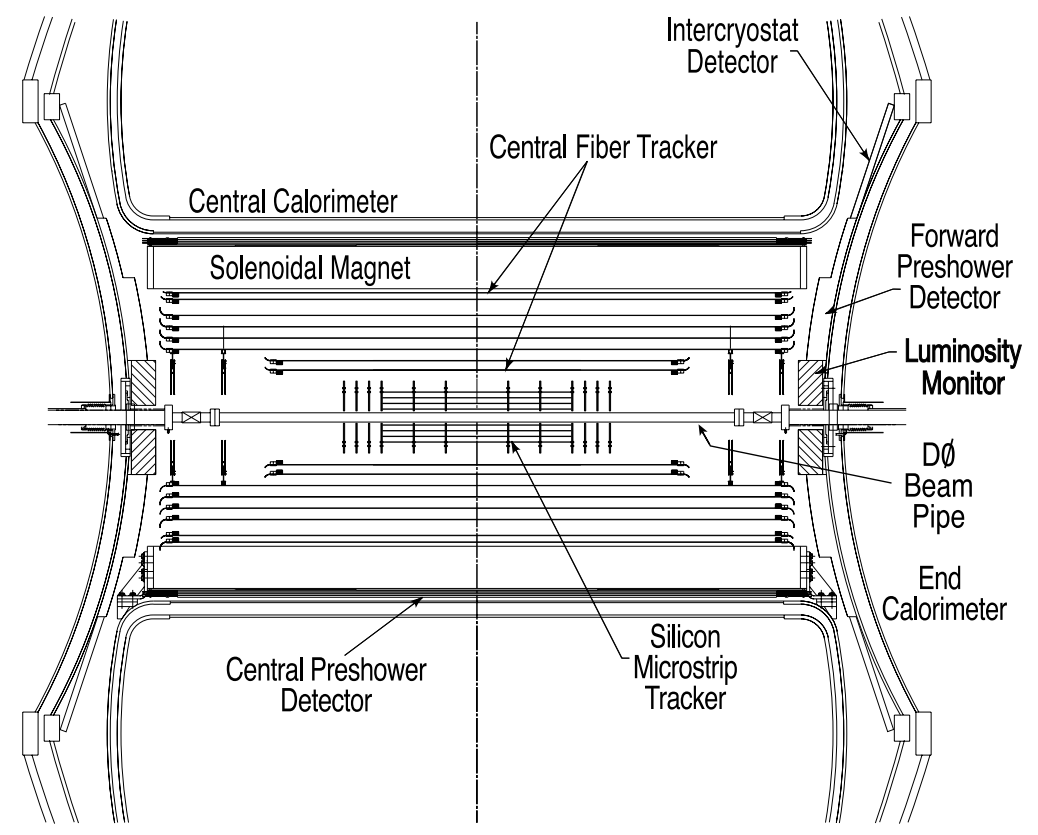

Figure 3.14: Side view of the central tracking detectors.

\subsubsection{The Solenoid}

The superconducting solenoid is based on super-conducting $\mathrm{Cu}$ :NbTi multi-filamentary cable strands that are stabilized by aluminum supports. The solenoid is $2.73 \mathrm{~m}$ in length and $1.42 \mathrm{~m}$ in diameter. The $2 \mathrm{~T}$ magnetic field is generated using a current of 4749 
A, and cooled using liquid Helium at $4.6 \mathrm{~K}$. Figure 3.15 shows the magnetic field lines from the solenoid. The polarity of the magnetic field is reversed periodically, but remains constant during a period of data taking (a store).

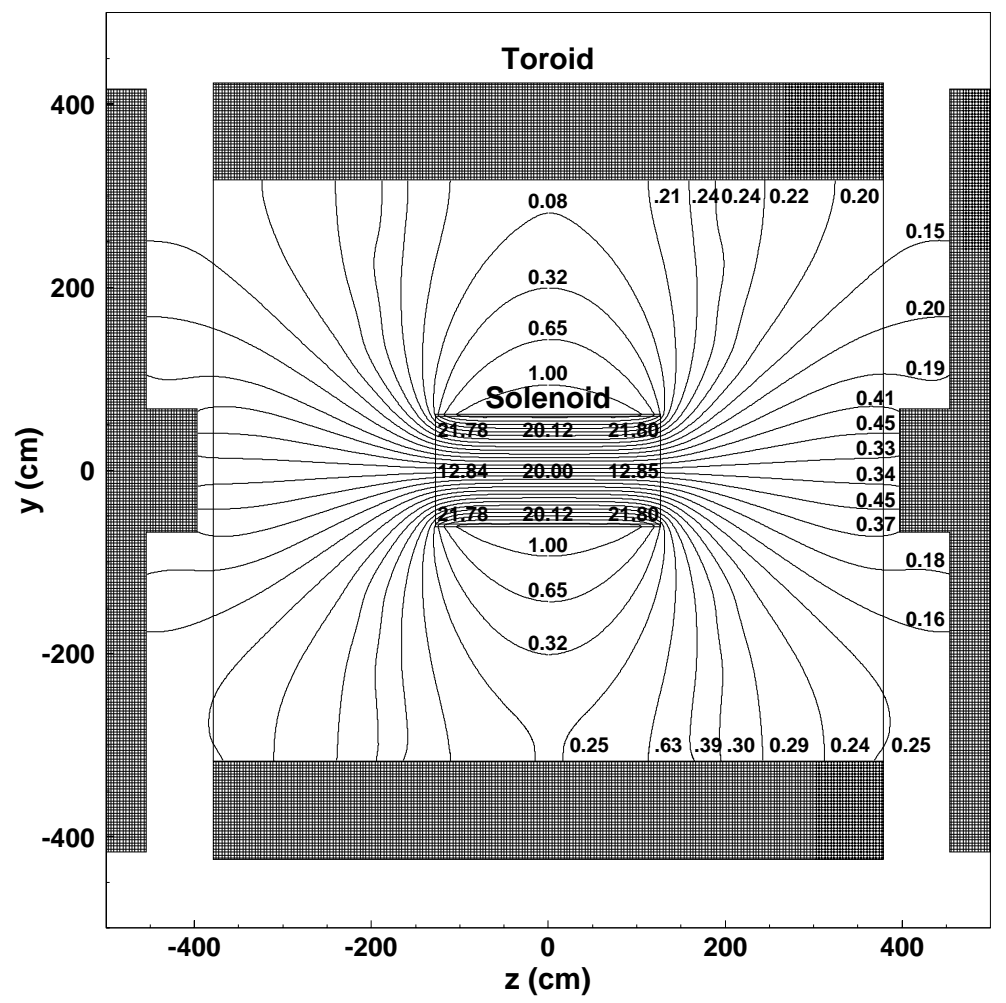

Figure 3.15: The magnetic field lines extend out to the magnetic toroid. The strength of the magnetic field is given in kiloGauss $(10 \mathrm{kG}=1 \mathrm{~T})$.

\subsubsection{The Silicon Microstrip Tracker Detector}

The silicon microstrip tracker (SMT) [27] is located just outside of the beryllium beam pipe, as shown in Figure 3.16. The SMT allows for track finding and vertex identification out to $|\eta|<3.0$. The tracker consists of doped silicon to produce p-n junction diodes operating at reverse bias. The p-n junctions form a depleted zone where there are no electrons in the conduction band. An applied reverse bias enhances the electric field across the depleted zone. Charged particles traversing the silicon will create electron hole pairs in the depleted zone which will be collected and produce a charge pulse. The charge 
is stored in capacitors and then read out and digitized. The SMT provides a precise measurement of the trajectory of charged particles. The axial hit resolution is $10 \mu \mathrm{m}$ and the $z$ hit resolution (for barrels) can be within $35 \mu \mathrm{m}$.

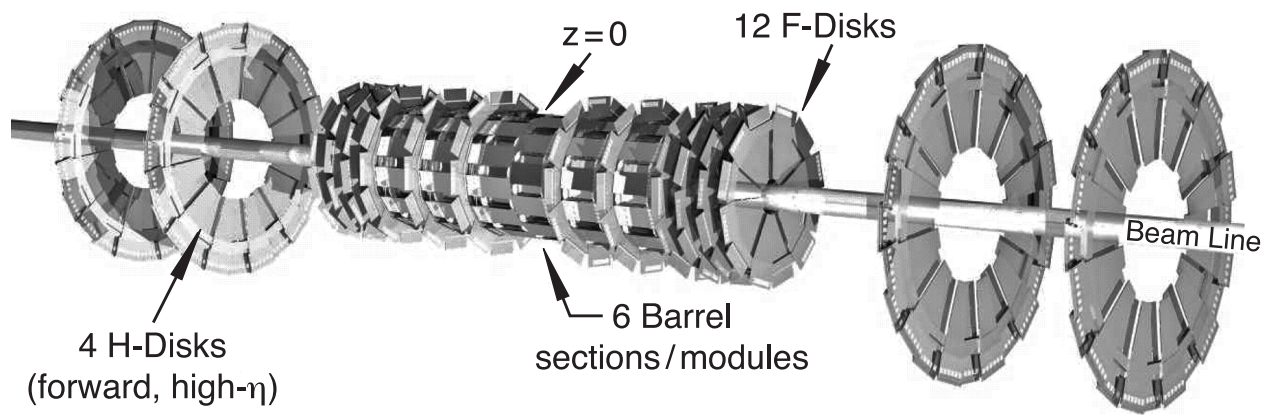

Figure 3.16: A 3D layout of the Silicon Microstrip Tracker.

The silicon wafers are formed into disks and barrels. The barrels are cylinders encompassing the beam pipe and measure the $(r, \phi)$ coordinates of charged particles, but have a large uncertainty in the $z$ direction. The disks are perpendicular to the beam pipe and can measure the $(r, \phi$, and $z)$ coordinates of the trajectory of a charged particle. There are six barrels centered symmetrically around $z=0$. At the end of each barrel there is an $\mathrm{F}$ disk. Each barrel consists of four readout layers, and each readout layer contains two staggered and overlapping sublayers, as shown in Figure 3.17. The F disks contain twelve double-sided wedge detectors (Figure 3.18). The third barrel is capped off with a $\mathrm{F}$ disk followed by three more $\mathrm{F}$ disks. Following the three $\mathrm{F}$ disks, there are two large diameter $\mathrm{H}$ disks which contain twenty-four pairs of single-sided detectors glued back to back. Table 3.1 shows the $|z|$ location of the center of each silicon detector. Table 3.2 shows the radii of the silicon detector components from the center of the beam pipe. The silicon microstrip tracker has a total of 792,567 readout channels. 


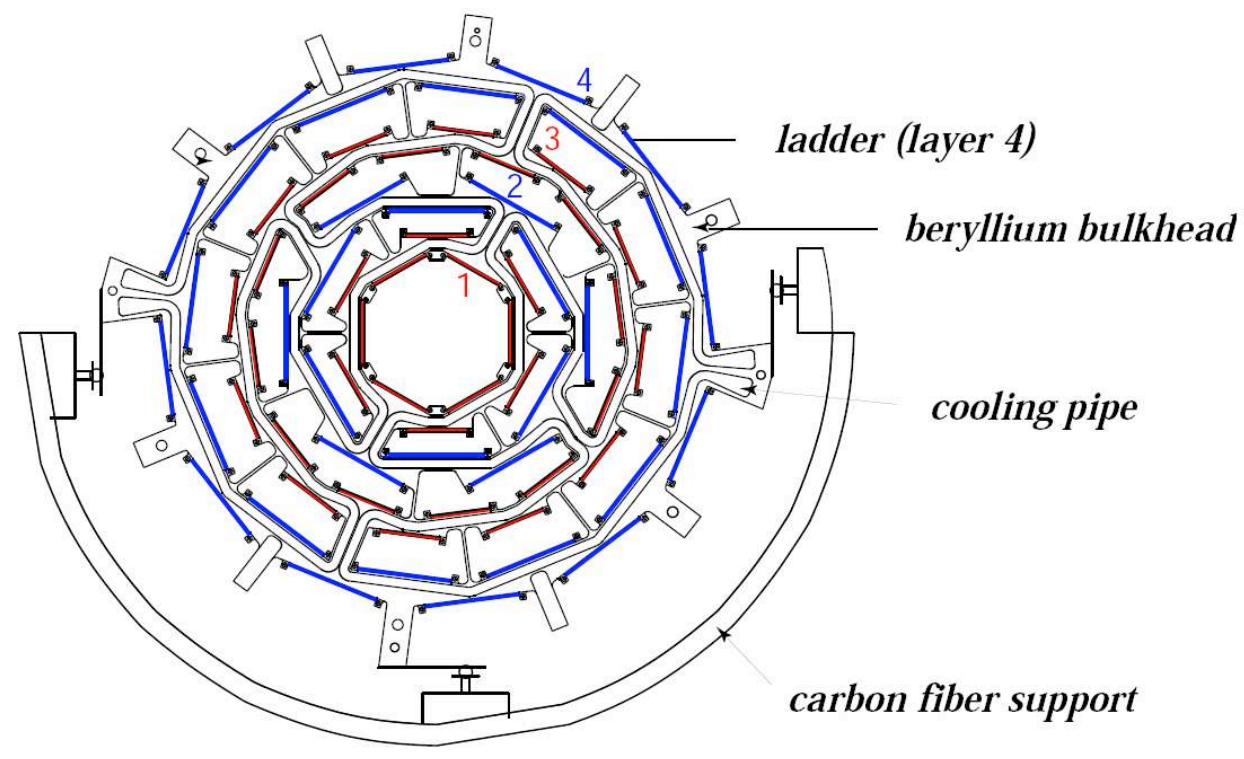

Figure 3.17: The $r-\phi$ view of the silicon barrels.

Table 3.1: Location of silicon microstrip tracker components along the $z$ direction starting from the center of the detector.

\begin{tabular}{|c|c|}
\hline unit & $|z|$ location $(\mathrm{cm})$ \\
\hline Barrel & $6.2,19.0,31.8$ \\
F disk & $12.5,25.3,38.2,43.1,48.1,53.1$ \\
H disk & $100.4,121.0$ \\
\hline
\end{tabular}

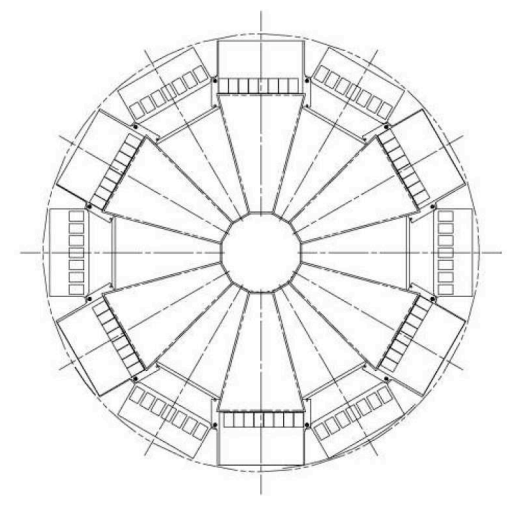

Figure 3.18: The $r-\phi$ view of the silicon wedge detectors of the F-disks.

Table 3.2: Silicon microstrip tracker detector component radii (measured from the center of the beam pipe).

\begin{tabular}{|c|c|}
\hline unit & radius $(\mathrm{cm})$ \\
\hline 4 Barrels & $2.7,4.6,7.6,10.5$ \\
F disk & 2.6 to 10.0 \\
H disk & 9.5 to 26 \\
\hline
\end{tabular}




\subsubsection{The Central Fiber Tracker Detector}

The central fiber tracker (CFT) [28] measures the position and momentum of charged particles using scintillating fibers. The ionizing particles excite atoms in the scintillating fibers. The excited atoms then emit light at $340 \mathrm{~nm}$ wavelength. These scintillating fibers have a dye which absorbs $340 \mathrm{~nm}$ light, and subsequently emits $530 \mathrm{~nm}$ light. The 530 $\mathrm{nm}$ wavelength light is extracted from the scintillating fibers using light pipes that direct the light onto the visible light photon counters (VLPC).

The pseudo-rapidity coverage of the CFT is $|\eta|<2.0$. The CFT has an inner radius of $19.5 \mathrm{~cm}$ and extends out to $51.5 \mathrm{~cm}$. It is composed of 76,800 scintillating fibers that form eight superlayers with increasing radius (Figure 3.19). Each superlayer is broken down into one axial layer (along the $z$ axis) and one stereo layer ( \pm 3 degrees). Each axial and stereo layer is further broken down into two layers (Figure 3.20). The inner layer (closest to the beam pipe) lies in a machined molding which separates the fibers by $926-990 \mu \mathrm{m}$ (depending on the superlayer). The outer layer fibers fit on top of two adjacent inner layer fibers. Two of the eight super layers are made of $166 \mathrm{~cm}$ long scintillating fibers. Two out of the eight super layers are made out of $252 \mathrm{~cm}$ scintillating fibers. Each superlayer can identify the $\eta$ and $\phi$ position of a charged particle within $100 \mu \mathrm{m}$. Each scintillating fiber is $835 \mu \mathrm{m}$ thick. The light leaving the scintillating fibers enters clear fiber waveguides and is turned into charge via impurity band silicon avalanche photodetectors. The Level-1 and Level-2 triggers (describes in Chapter refchap:Triggers) use hits from the axial layers. The Level-3 trigger uses axial and stereo information.

\subsubsection{The Preshower Detectors}

The scintillating fiber preshower detectors [29] [30] are located in between the solenoid and the calorimeter, shown in Figure 3.21 and Figure 3.25. They are designed to im- 


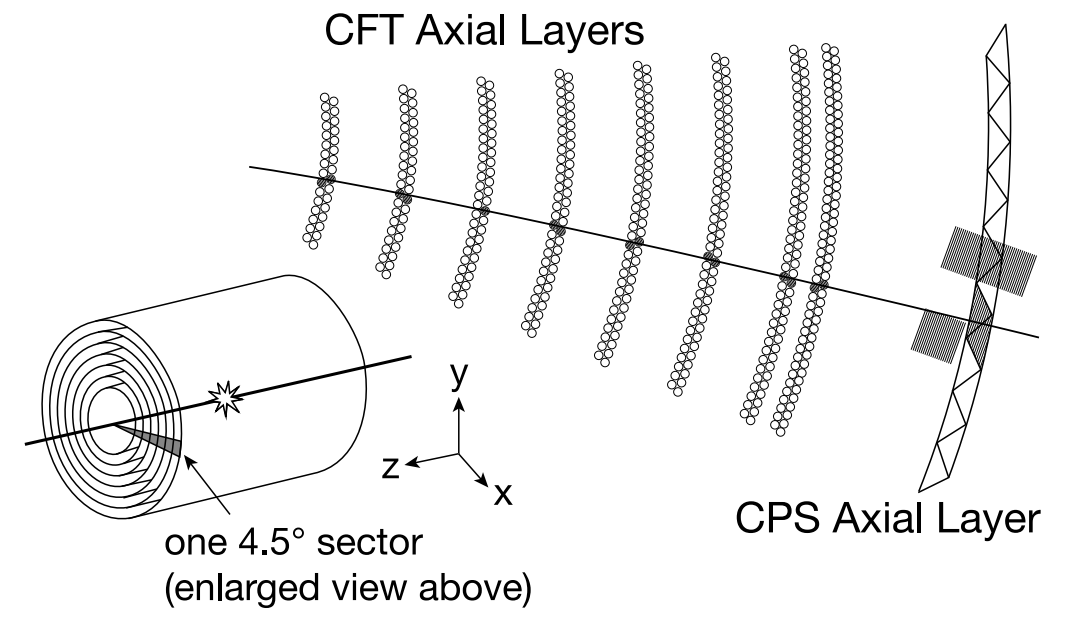

Figure 3.19: The central fiber tracker located between the silicon microstrip tracker and the solenoid. The cross section blow up of the fibers from two layers.

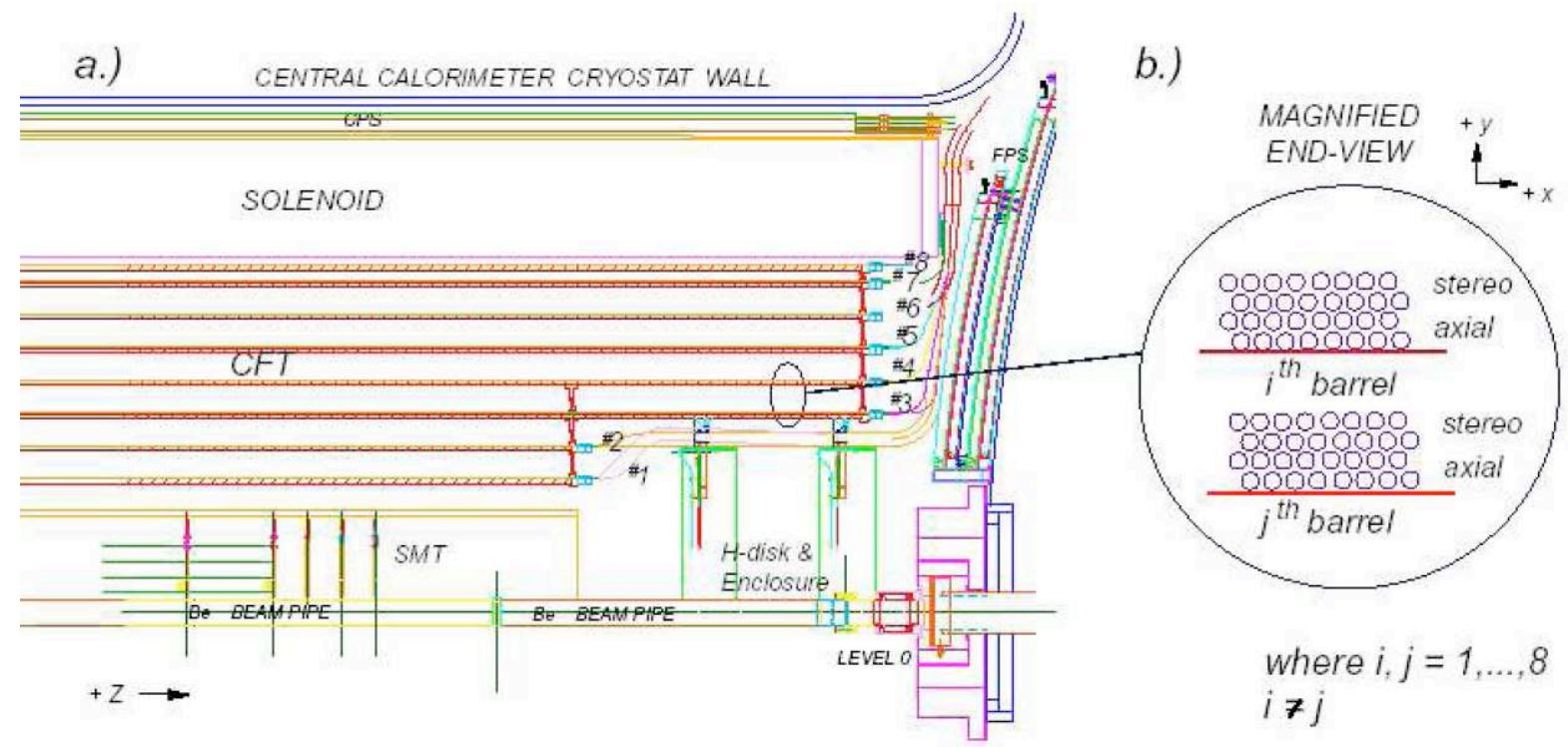

Figure 3.20: a) The $r-z$ diagram of the central fiber tracker. b) A cross section view of the fibers. 
prove the identification and energy measurement of electrons and photons. There are two preshower detectors: a central detector covering $|\eta|<1.3$ and two forward detectors covering $1.5<|\eta|<2.5$. The scintillating fibers are triangular strips, individually wrapped in aluminized mylar, epoxied and interlocked together as shown in Figure 3.23. This arranges scintillator layers without creating any dead space and thereby improves the accuracy of position measurements. The center of each strip contains a $835 \mu \mathrm{m}$ diameter wavelength shifting fiber that collects and carries the light to the clear fiber light-guide, which is connected to the VLPCs. The other end of the triangle strip is coated with silver. The visible light leaves the clear fiber waveguides and is turned into electric charge by a $2 \times 4$ array of $1 \mathrm{~mm}$ diameter pixel impurity band silicon avalanche photodetectors. There are 22,564 readout channels for the preshower detectors.

\subsubsection{Central Preshower}

The central preshower detectors (CPS) is sandwiched between the solenoid and the central calorimeter and covers $|\eta|<1.3$. After the solenoid, there is a lead radiator covered by a stainless steel skin) which has a thickness of one radiation length $\left(\chi_{0}\right)^{2}$. The CPS is broken up into two separate units, north and south of $z=0$. Each side has three layers of triangular shaped fibers. The inner most layer lies along the $z$ axis, the next layer lies along the $z$ axis at a stereo angle of +23.8 degrees $(u)$, and the final layer lies along the $z$ axis at a stereo angle of -24.0 degrees (v), as shown in Figure 3.21. Each of the three layers, axial, $\mathrm{u}$, and $\mathrm{v}$ consist of 1280 strips. The central preshower detector is subdivided into $80 \phi$ sectors of Level-1 triggering, therefore 16 fibers (per layer) makeup one $\phi$ sector. Only the axial layers are used for Level-1 triggering, but all three layers are used for Level-2 triggering.

\footnotetext{
${ }^{2}$ The mean distance over which a high-energy electron losses all but 1/e of its energy by Bremsstrahlung, and equivalently it is the $\frac{7}{9}$ of the mean free path for pair production by high energy photons.
} 


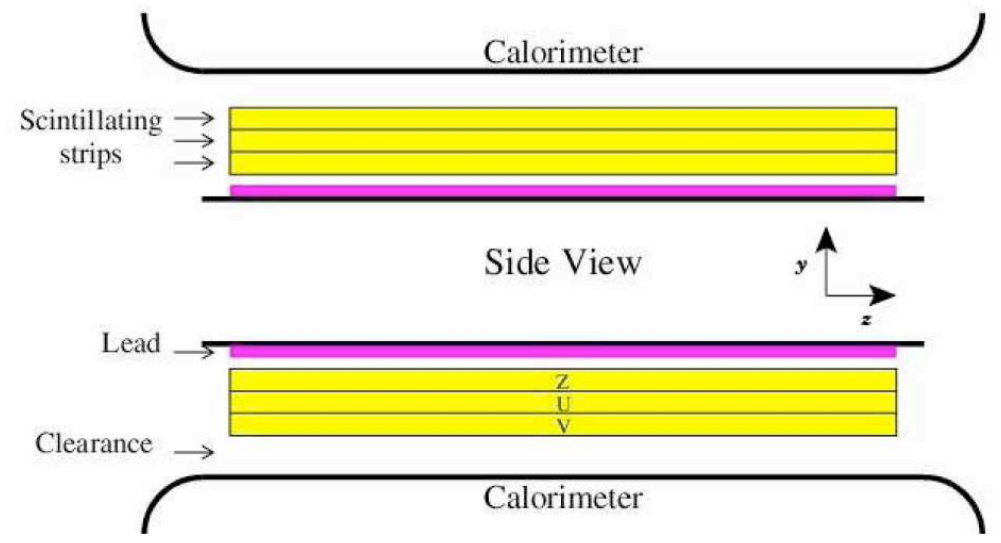

Figure 3.21: Schematic $r-z$ side view of the central preshower detector.

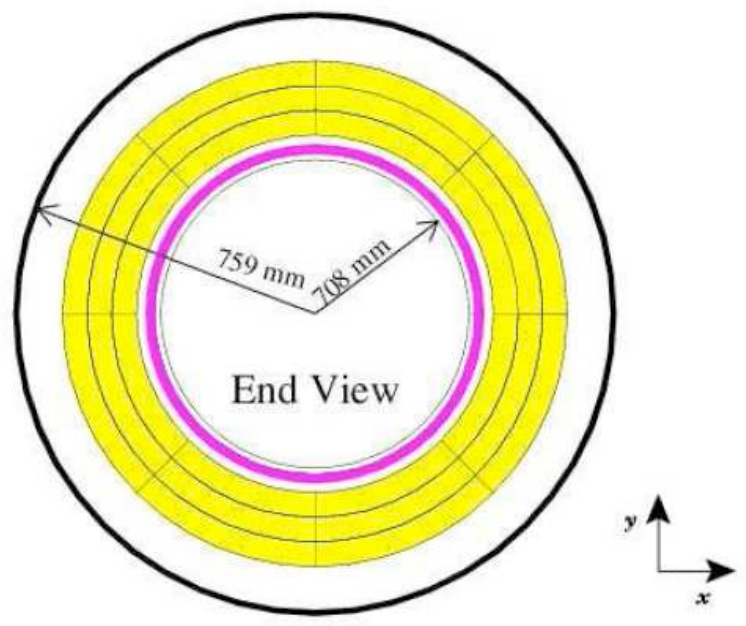

Figure 3.22: Schematic $r-\phi$ view of the central preshower detector.

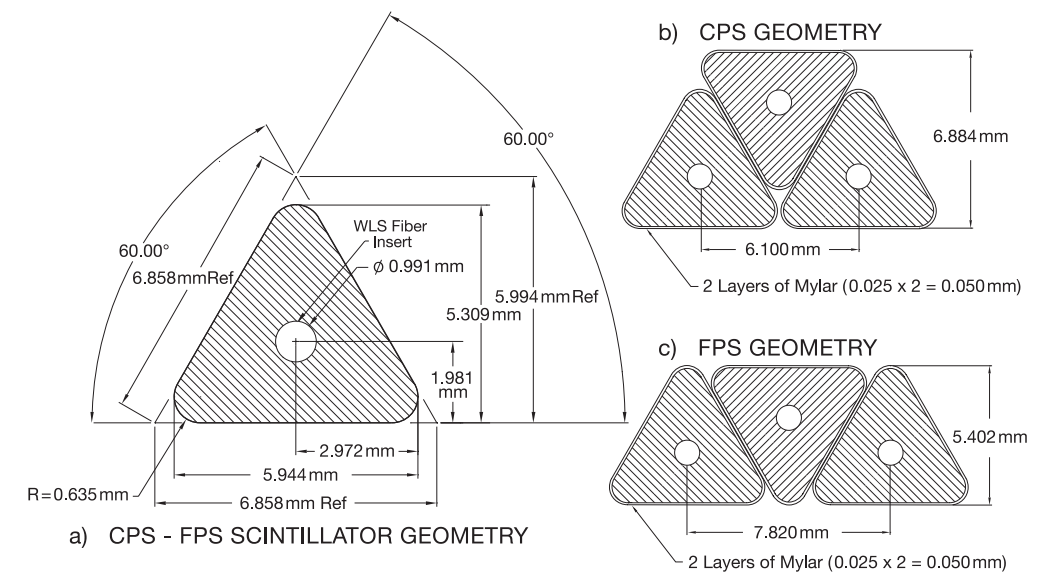

Figure 3.23: The cross section of the central and forward preshower scintillating triangular strips. 


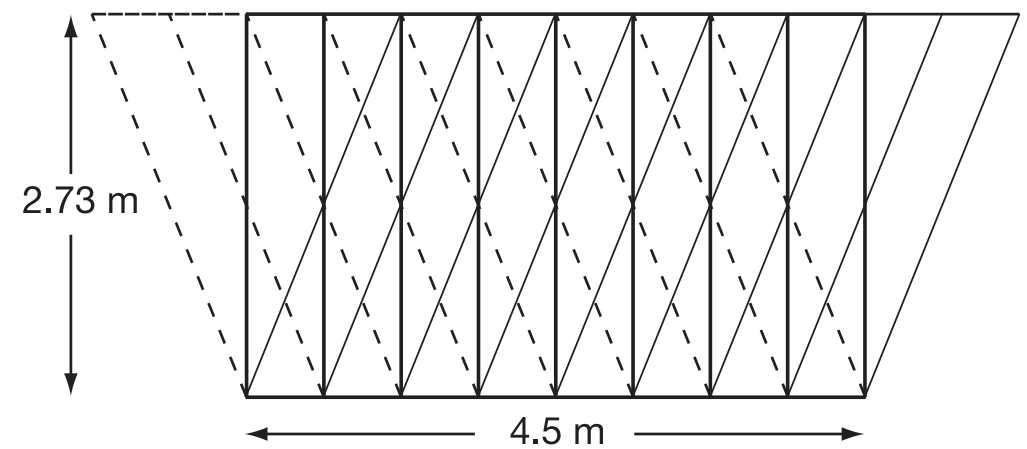

Figure 3.24: The layout of the three layers of scintillating strips - one axial and two at stereo angles.

\subsubsection{Forward Preshower}

The forward preshower detectors (FPS) are broken up into two units that are attached to the two end calorimeters covering $1.5<|\eta|<2.5$. There are four layers of triangular shaped scintillating strips in the detector which are broken up into two regions: minimum ionizing particle (MIP) and shower. Sandwiched between the MIP and the shower regions is $2 \chi_{o}$ thick lead absorber plate covered in stainless steel. The MIP region locates the $\eta$, $\phi$, and $z$ position of the trajectory of the charged particle while the shower layer measures the energy of the particle. A photon will not deposit energy in the MIP region but it may interact with the lead absorber depositing energy in the shower region. The two MIP layers have $\mathrm{u}$ and $\mathrm{v}$ stereo fibers covering $\Delta \phi=22.5$ degrees. The shower layers have a similar configuration. The detector is broken up into sixteen 22.5 degree wedges, as shown in Figure 3.25. One 22.5 degree wedge contains 1u MIP layer, an absorber, and $1 \mathrm{v}$ shower layer. The next 22.5 degree wedge contains $1 \mathrm{v}$ MIP layer, an absorber, and $1 \mathrm{u}$ shower layer. There are 7442 channels on each of the two forward preshower detectors.

\subsubsection{The Calorimeters}

A calorimeter is a detector that intercepts the primary particle and is of sufficient thickness to cause it to interact and deposit most of its energy inside the detector volume 


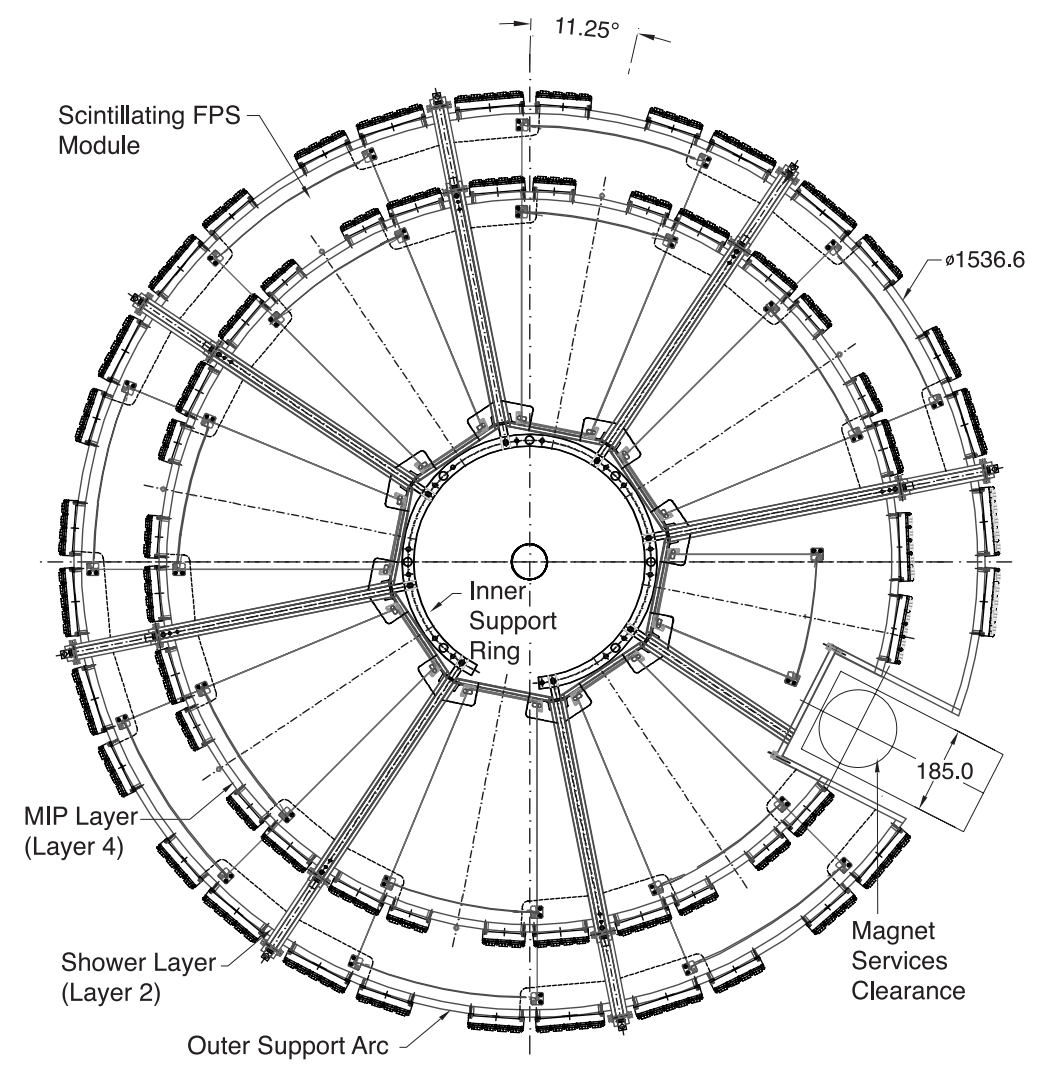

Figure 3.25: The forward preshower detectors attached to each of the calorimeter end caps. 
in a subsequent cascade or "shower" of increasingly lower-energy particles [31]. The main purpose of the calorimeter is to measure the energy and position of electrons, photons, and jets from $\bar{p} p$ collisions. The electrons and photons will shower in the calorimeter into lower energy electrons and photons with a transverse width of about $1-2 \mathrm{~cm}$ (Moliere radius). Hadrons interact with the nucleus of the absorber material creating a cascade of secondary particles. Half of the incident hadron energy is carried by low $p_{T}$ particles with the remainder of the energy carried by a few fast particles continuing the cascade. The transverse width of a hadron shower is typically $10 \mathrm{~cm}$.

The calorimeter (Figure 3.26) cover the pseudo-rapidity range of $|\eta|<4.0$. The calorimeter is broken up into three different detectors, the central calorimeter (CC) and two end calorimeters (EC). Each detector is housed within its own cryostat and maintained at $90 \mathrm{~K}$. The CC covers $|\eta|<1$. Each calorimeter is broken up into two sections: the EM section and the hadronic section. The hadronic section is further broken down into degrees of coarseness of the absorber plates. The central calorimeter has a fine hadronic region where the absorber plates (copper) are $46.5 \mathrm{~mm}$ thick. The end calorimeters have an EM absorber section and three hadronic absorber regions: inner, middle, and outer.

A typical unit cell is depicted in Figure 3.27. The unit cell contains an absorber plate, liquid argon gap, a signal board, followed by a liquid argon gap. The absorber plates are $3-46.5 \mathrm{~mm}$ thick and held at zero voltage. The signal board is made of two copper-clad G10 sheets laminated together with a resistive epoxy coating covering the two surfaces that are exposed to the liquid argon. The two epoxy covered surfaces are held at around 2 $\mathrm{kV}$. One G10 sheet has the inner copper-clad coating removed, while the other G10 sheet has its inner copper-clad coating milled for segmented readout. Liquid argon fills the 2.3 mm gap between the absorber and signal boards. Charged particles passing through the liquid argon form ions causing current to flow across the potential difference. A group 


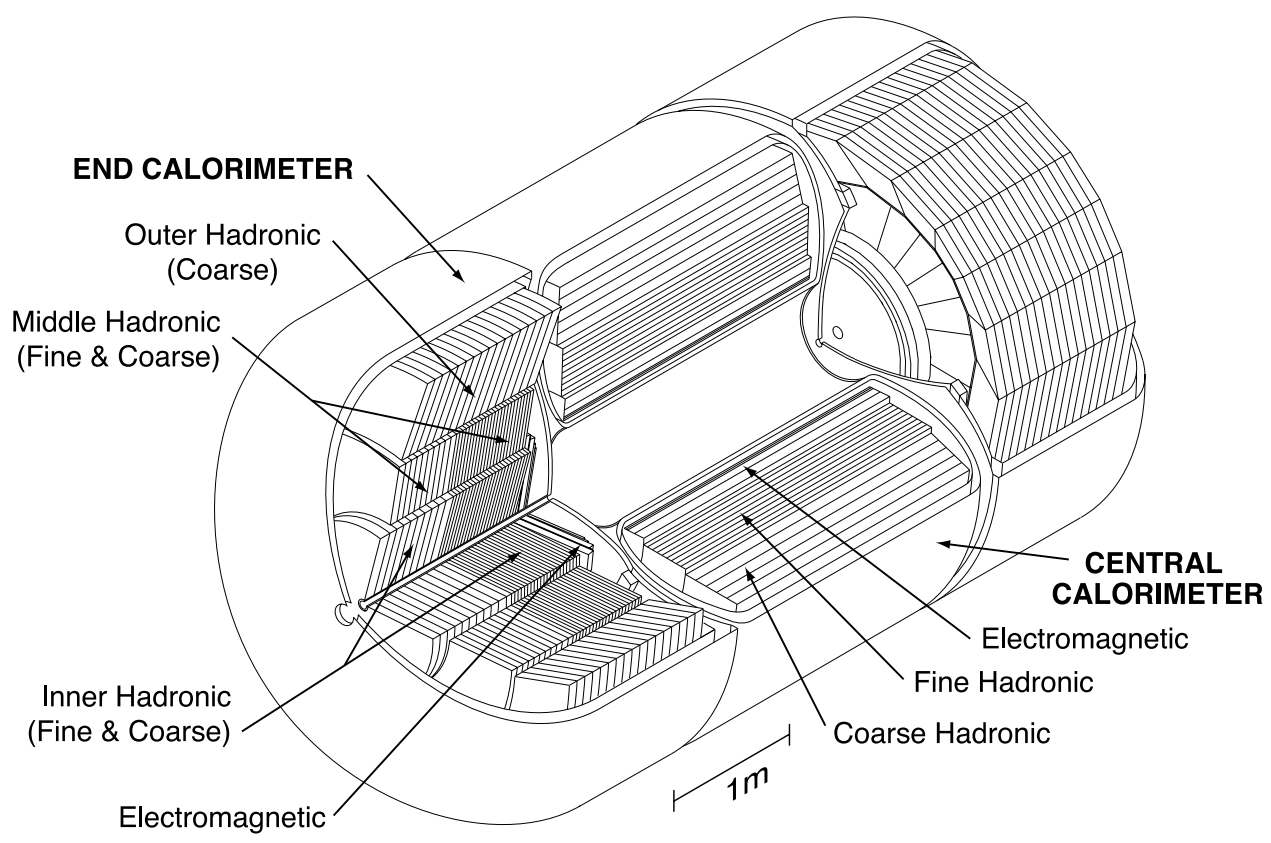

Figure 3.26: A view of the central and end cap calorimeters.

of unit cells along the same trajectory form a layer. All layers along the same trajectory form a calorimeter readout tower of $0.1 \times 0.1$ in $\eta \times \phi$ (Figure 3.28 and Figure 3.29).

Table 3.3 shows the parameters for the CC. The EM section of the CC has four readout layers. The first two readout layers are made of two unit cells each. The third readout layer has seven unit cells while the fourth layer has ten unit cells. The fine hadronic section for the CC has three readout layers with twenty, sixteen, and fourteen unit cells, respectively. The coarse hadronic sections has one readout layer consisting of nine unit cells. 3.4 shows the detectors parameters for the EC.

Table 3.3: Presented are the calorimeter detector parameter values.

\begin{tabular}{|c|c|c|c|}
\hline & EM & FH & CH \\
\hline Number of modules & 32 & 16 & 16 \\
Absorber $^{3}$ & $\mathrm{U}$ & $\mathrm{U}-\mathrm{Nb}$ & $\mathrm{Cu}$ \\
Absorber thickness $(\mathrm{mm})$ & 3 & 6 & 46.5 \\
Argon Gap (mm) & 2.3 & 2.3 & 2.3 \\
Number of readout layers & 4 & 3 & 1 \\
Cells per readout layer & $2,2,7,10$ & $20,16,14$ & 9 \\
Total radiation lengths $\left(X_{o}\right)$ & 20.5 & 96.0 & 32.9 \\
Total nuclear absorption lengths $(\lambda)$ & 0.76 & 3.2 & 3.2 \\
Total readout cells & 10368 & 3000 & 1224 \\
\hline
\end{tabular}


Table 3.4: Presented are the end calorimeter parameters.

\begin{tabular}{|c|c|c|c|c|c|c|}
\hline & EM & IFH & ICH & MFH & MCH & OH \\
\hline Number of modules & 1 & 1 & 1 & 16 & 16 & 16 \\
Absorber & $\mathrm{U}$ & $\mathrm{U}-\mathrm{Nb}$ & $\mathrm{SS}$ & $\mathrm{U}-\mathrm{Nb}$ & $\mathrm{SS}$ & $\mathrm{SS}$ \\
Absorber thickness $(\mathrm{mm})$ & 4 & 6 & 46.5 & 6 & 46.5 & 46.5 \\
Argon Gab $(\mathrm{mm})$ & 2.3 & 2.1 & 2.1 & 2.2 & 2.2 & 2.2 \\
Number of readout layers & 4 & 4 & 1 & 4 & 1 & 3 \\
Cells per readout & $2,2,6,8$ & 16 & 14 & 15 & 12 & 8 \\
Total radiation lengths $\left(X_{o}\right.$ & 20.5 & 121.8 & 32.8 & 115.5 & 37.9 & 65.1 \\
Total Nuclear absorption lenghts $(\lambda)$ & 0.97 & 4.9 & 3.6 & 4.0 & 4.1 & 7.0 \\
Total readout cells & 7488 & 4288 & 928 & 1472 & $384+64$ & 896 \\
\hline
\end{tabular}

The EM detector for the central calorimeter is made up of thirty-two modules along $\phi$. The EM modules boundaries, called " $\phi$ cracks", are uninstrumented regions. Electrons and photons passing through the $\phi$ cracks only deposit a fraction of their energy amongst the two adjacent $\phi$ modules. The fine hadronic section is broken up into sixteen modules along $\phi$ and centered on the EM module boundaries.

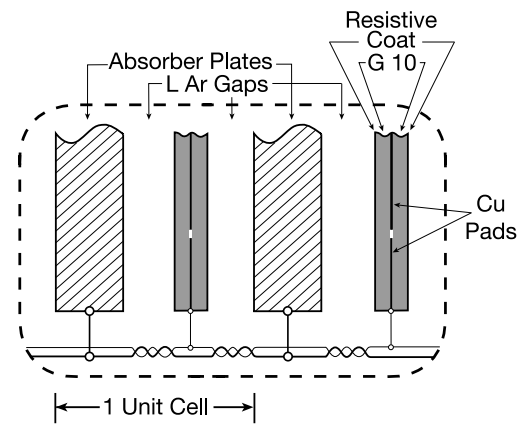

Figure 3.27: Schematic view of a unit cell in the calorimeter.

The four EM layers at $\eta=0$ have radiations lengths $\left(\chi_{0}\right)$ of $1.4,2.6,6.8$, and 9.8 respectively, totaling $20 \chi_{0}$ and 0.76 interaction lengths $\left(\lambda_{l}\right)$. The hadronic layers at $\eta=0$ have a total of $129 \chi_{0}$ and $6.4 \lambda_{l}$ of material. The $\mathrm{D} \varnothing$ calorimeter was designed to separate EM and hadronic showers by using a high absorber material (depleted uranium).

$$
\frac{\lambda_{l}\left(\mathrm{~g} / \mathrm{cm}^{2}\right)}{\chi_{0}\left(\mathrm{~g} / \mathrm{cm}^{2}\right)} \sim \frac{35 A^{\frac{1}{3}} Z^{2}}{180 A} \sim 0.12 Z^{\frac{4}{3}}
$$




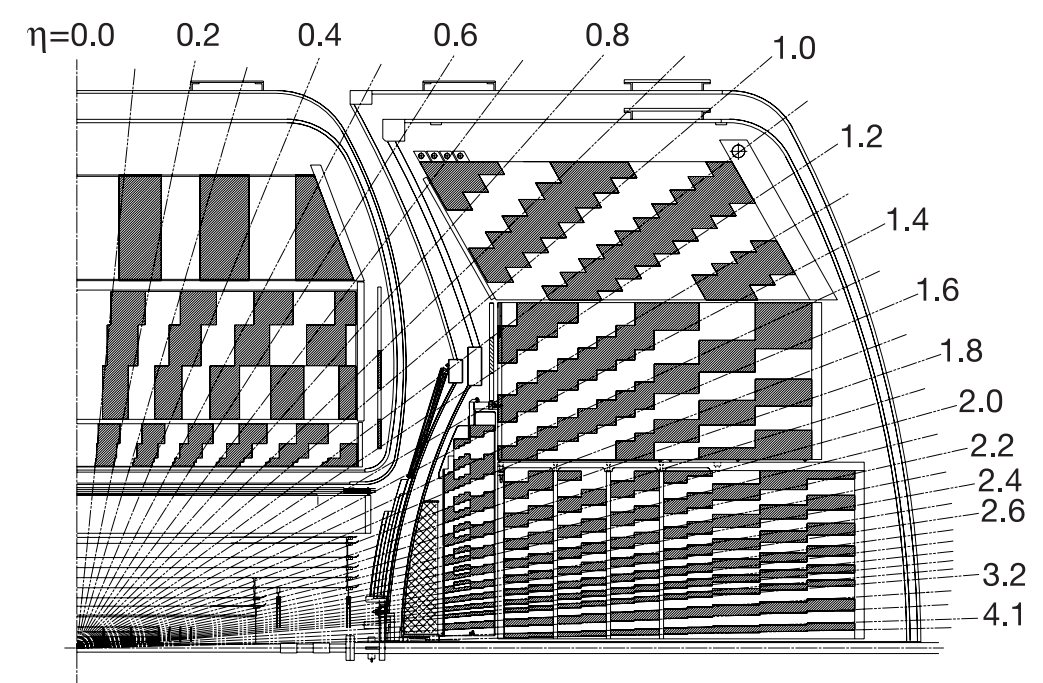

Figure 3.28: One quarter view of the calorimeter showing the segmented readout.

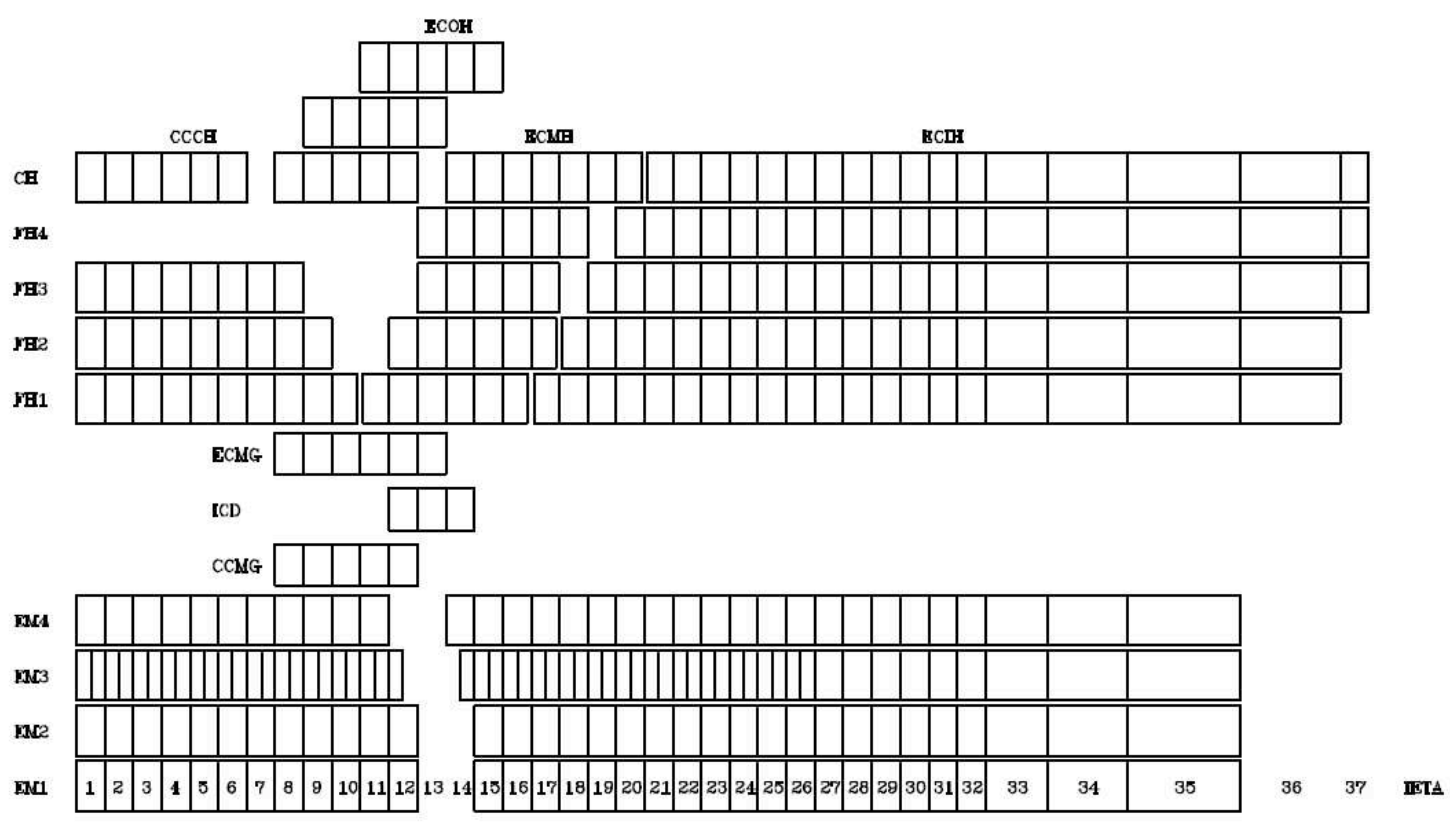

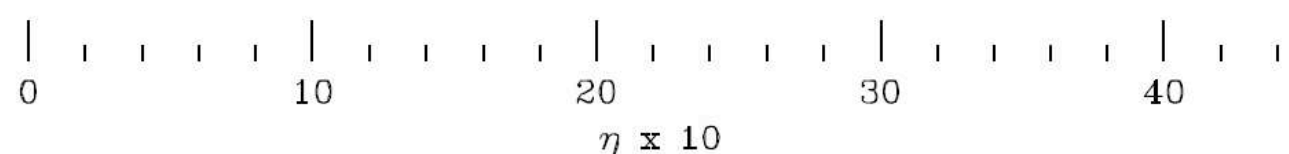

Figure 3.29: The calorimeter readout towers in $\eta$ versus the layers distributed along the shower development. 
The three calorimeter detectors have under-instrumented regions called the inter cryostat regions (ICR) caused by the physical boundaries of the cryostats. To improve the energy measurements, two additional types of detectors are added to the calorimeters. The first is a ring in $\phi$ of massless gap detectors covering $0.6<|\eta|<1.3$. The massless gap detectors are located inside the cryostats but outside of the absorber plate region. The massless gap detectors are similar to the unit cell detectors but they do not have absorber plates. The second ICR detector is a ring in $\phi$ of scintillator tiles covering $1.1<|\eta|<1.5$. The tiles are arranged to give segmented readout comparable to match a unit cell.

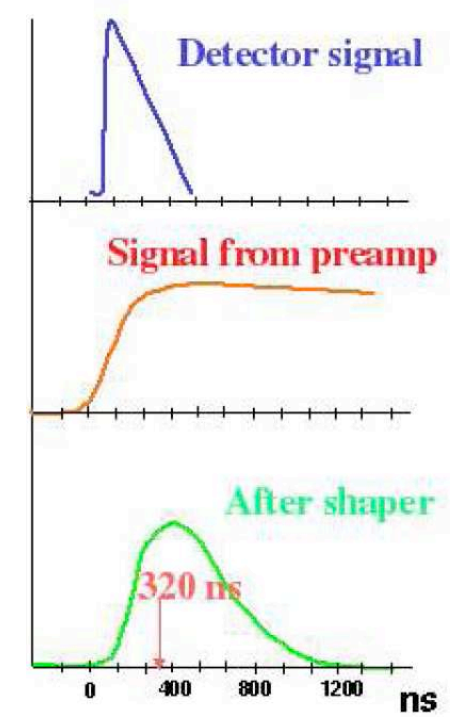

Figure 3.30: The shape of the signals detected and processed through the electronics in the calorimeter.

The readout of the calorimeter layers is characterized by a pre-amplifier, a Level-1 trigger pick-off, signal shaping, storage circuits, and analog to digital converter (ADC). The calorimeter electronics samples the shaped signal every 132 ns. The electron drift velocity across the $2.3 \mathrm{~mm}$ gap at $2 \mathrm{kV}$ is approximately $450 \mathrm{~ns}$. The pre-amplifier has a rise time of $450 \mathrm{~ns}$ and a decay time of $15 \mu \mathrm{s}$. The pre-amplifier signal does not fall quickly enough for the voltage to be at zero for the next collision. This signal tail produces a baseline DC offset for the next interaction, Figure 3.30. The pre-amplifier signal is sent 
to the Base Line Subtractor (BLS) board which can hold the signal for $4 \mu$ s, waiting for a Level-1 trigger decision (Figure 3.31). The BLS boards provide base line subtraction of the pre-amplifier signal, and fast shaped analog sums of the signal for Level-1 and Level-2 trigger decisions. The fast pick-off analog sums arrange EM and EM+hadronic energies in towers of $0.2 \times 0.2$ in $\Delta \eta \times \Delta \phi$. Only $2 / 3$ of the pre-amplifier signal or $260 \mathrm{~ns}$ is used for the signal shaper circuit. The shaped signal has a peak at 320 ns with a 1.2 $\mu$ s decay. After a Level-1 accept ${ }^{5}$ the signal is transferred into a forty event deep buffer before transfer to Level-3.

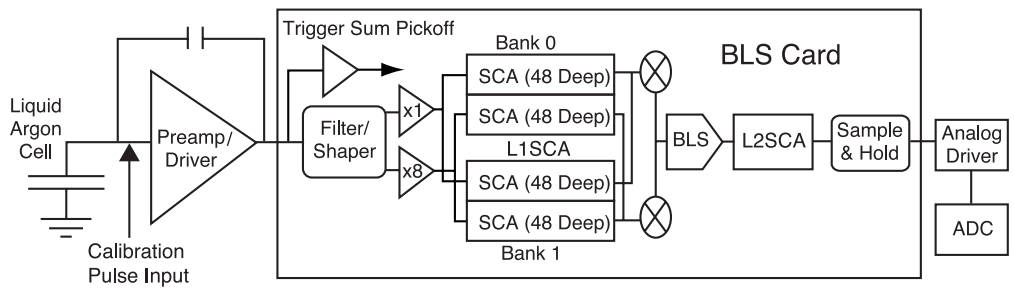

Figure 3.31: The electronic readout of the unit cell to the analog to digital converter.

\subsubsection{The Muon System}

The muon spectrometer [32], [25] is designed to detect muons, which are minimum ionizing particles at transverse momenta greater than $1 \mathrm{MeV}$. Therefore, the muons penetrate through all of the detectors and only leave small energy deposits in the tracking system, calorimeter, and muon spectrometer. The muon spectrometer consists of proportional drift tubes, mini drift tubes, scintillator counters, and a $1.8 \mathrm{~T}$ iron toroid magnet. The muon-detector system has three layers: A, B, and C. The A layer is in between the calorimeter and the toroid. The B and C layers are located outside of the toroid (Figure 3.34). The scintillator counters mounted inside (layer A) and outside (layers B and C).

The proportional drift tubes (PDT) detect the ionization charge when the muons interact with the gas inside the tubes. The $10.1 \mathrm{~cm}$ wide tubes are rectangular in shape

\footnotetext{
${ }^{5}$ Described in Chapter 4
} 


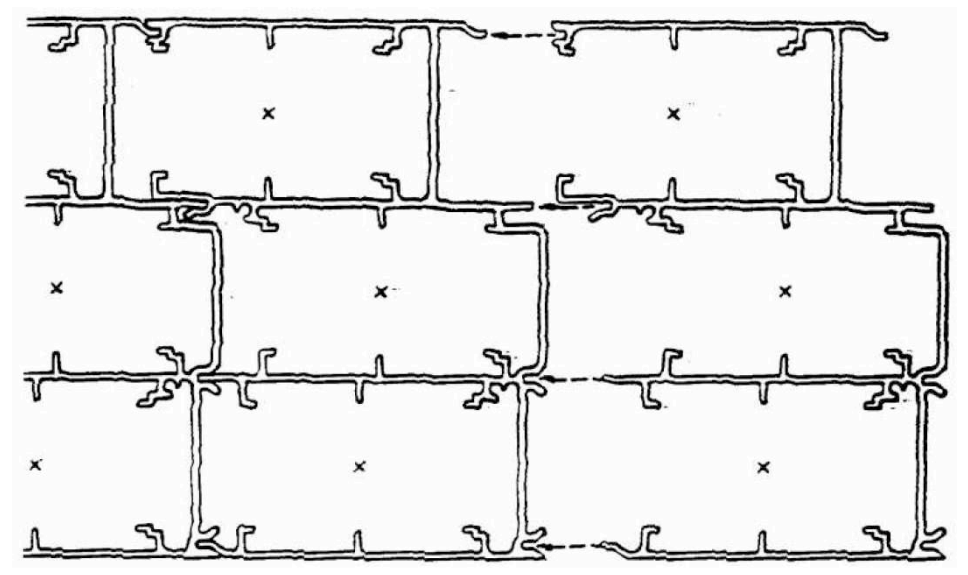

Figure 3.32: Cross section through proportional drift chambers.

and have a $50 \mu \mathrm{m}$ gold plated tungsten wires parallel to the toroid magnetic field (Figure 3.32). An anode wire is located at the center of each tube and held at $4.7 \mathrm{kV}$. Along the inner walls of the tube are vernier cathode pads, which are placed above and below the wire and held at $2.3 \mathrm{kV}$. The gas $\left(84 \% \mathrm{Ar}, 8 \% \mathrm{CF}_{4}\right.$, and $\left.8 \% \mathrm{CH}_{4}\right)$ is allowed to flow through the tubes. The maximum electron drift time is $500 \mu \mathrm{s}$. The hit resolution of the PDTs is $5 \mathrm{~mm}$. The A layer consists of four layers of drift tubes, while the B and C layers consists of three layers of drift tubes.

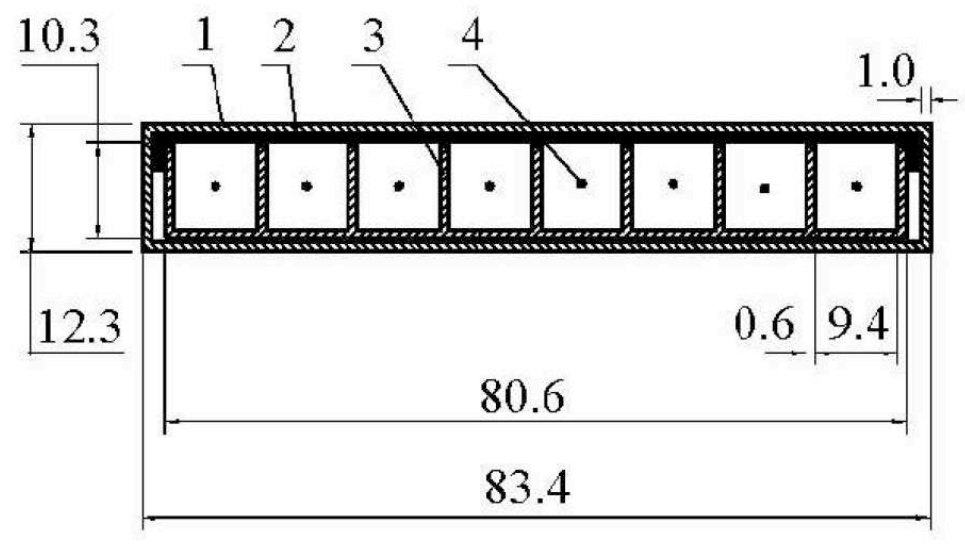

Figure 3.33: A cross section of the mini-drift tubes.

The mini drift tubes (MDT) are similar to the proportional drift tubes. The MDTs are 
smaller, $9.4 \times 9.4 \mathrm{~mm}^{2}$, do not have vernier cathode pads, and contain a slightly different mixture of gas $\left(90 \% \mathrm{CF}_{4}\right.$, and $\left.10 \% \mathrm{CH}_{4}\right)$. The MDTs are in the forward muon system covering $1.1<|\eta|<2.2$. The tubes contain a $50 \mu \mathrm{m}$ gold plated tungsten wire held at ground voltage(Figure 3.33).

The scintillation counters are used for position measurements and a precise measurement of the muon arrival times. Cosmic ray muons are rejected using arrival times of the muons. The scintillators are half inch thick and are made of Bicron 404A scintillator with a wave-shifting fiber placed in a milled out groove on the scintillator. Photomultiplier tubes are attached to the ends of the wave-shifter fibers for readout. The fast response and readout of the scintillation counters are used for Level-1 trigger dections. The scintillators are segmented into regions of 4.5 degrees matching the CFT segmentation. The CFT tracks and muon scintillator hits are matched for Level-1 trigger decisions.

The toroid magnet is broken down into six separate sections forming a cube around the calorimeter. The toroid is a square annulus of $109 \mathrm{~cm}$ thickness. The inner surface is $318 \mathrm{~cm}$ from the beam line. The iron magnet is wound using coils of ten turns each. A current of $1500 \mathrm{~A}$ is applied to the coils and the resulting magnetic field is $1.8 \mathrm{~T}$. Figure 3.34 shows a simulated deflection of a muon through the magnetic field produced by the toriod. 


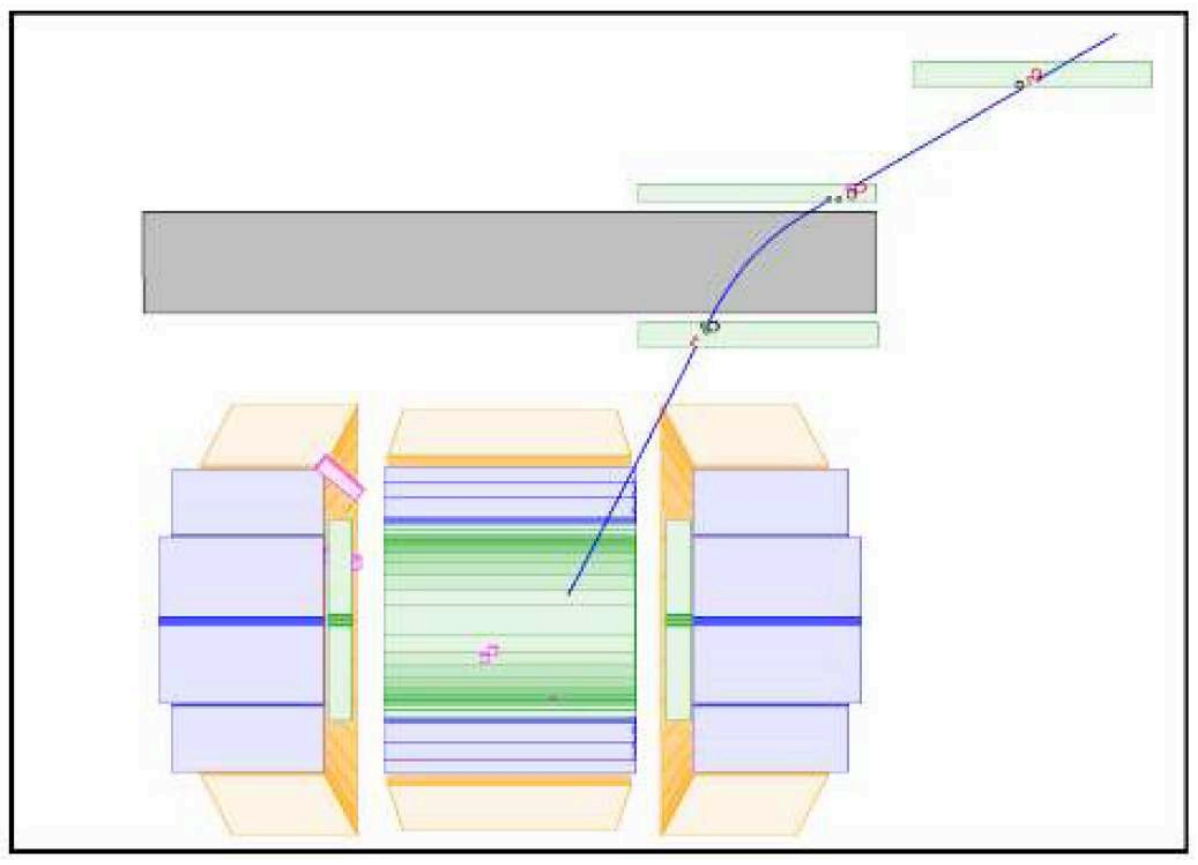

Figure 3.34: Simulated muon trajectory through the $\mathrm{D} \varnothing$ detector. 


\section{CHAPTER 4}

\section{The DØ Trigger and Data Acquisition Systems}

\subsection{Trigger Overview}

The Tevatron collides 36 bunches of anti-protons at a collision rate of $1.7 \mathrm{MHz}$. The average amount of data collected by the $\mathrm{D} \varnothing$ detector per event is $250 \mathrm{kB}$. Collecting data from every collision would require storing approximately 425 GB of data per second which is not feasible. In addition, interesting high energetic $\bar{p} p$ collisions have a low probability to occur. The selection of such collisions from the elastic and soft inelastic events is done via a three level trigger system [33]. The Level-1 (L1) trigger system [34] uses a hardware trigger to reduce the rate by a factor of 1000. The Level-2 (L2) trigger system [35], [36] reduces the rate by a factor of 2-5, and the Level-3 (L3) trigger system [37] reduces the rate by a factor of 10 . The data are stored on local online disks at an average of $50-100$ $\mathrm{Hz}$ for further transmission to permanent tape storage. The amount of data stored per second is about $12.5 \mathrm{MB}$.

The data acquisition is controlled by the COOR [38] software package (Figure 4.1). COOR receives the trigger list, communicates with the trigger framework, relays the trigger decision parameters, and controls the data flow. The trigger list specifies the requirements which can fire an event at each trigger level. The trigger list contains about 450 L3 trigger conditions that correspond to specific L1 and L2 trigger bits. The L1 and 
L2 systems have a one-to-one correspondence of the 128 possible trigger bits.

Figure 4.1 shows the flow of data from the detector to storage on tape. With a beam crossing rate of $1.7 \mathrm{MHz}$, the detector information is passed to the L1 trigger. Once a L1 condition is satisfied, the trigger framework (TFW) sends a L1 accept to the detector sub-systems, and the detector information is sent to the L2 system. Once the L2 trigger generates a decision, it passes its decision to the TFW. The TFW then communicates to the detector sub-systems to send the data to L3. The data acquisition system (DAQ) is responsible for the coordination of the L3 farm nodes and the online run control. Once the data is inside a L3 farm node, the event is processed through a nearly complete event reconstruction and the final trigger decision is made.

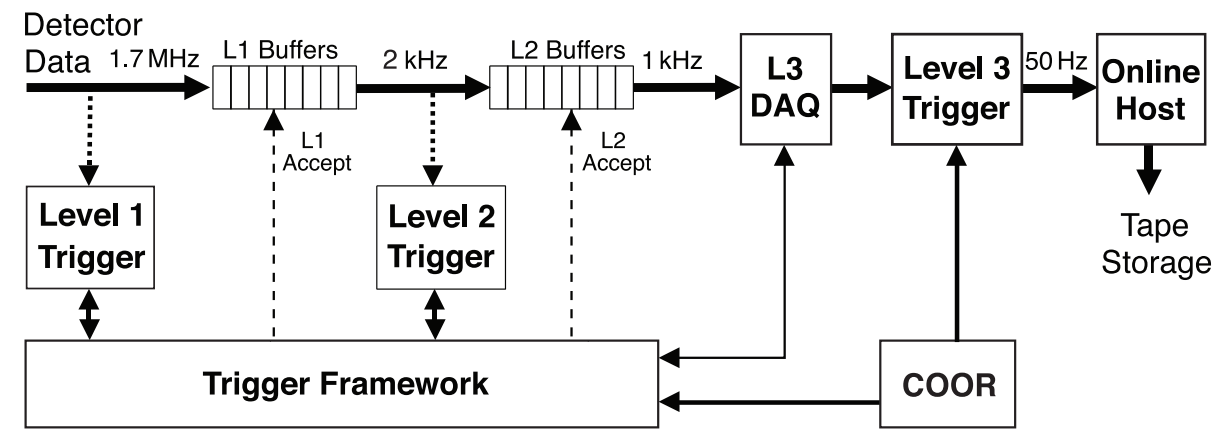

Figure 4.1: The flow of the data from the $\mathrm{D} \varnothing$ detector to storage onto magnetic tapes.

\subsection{The Level-1 Trigger}

The L1 trigger (Figure 4.2) generates decisions based on requirements from the tracking, calorimeter, and muon detectors. The L1 trigger decision, made by the TFW, is based on 128 individual trigger conditions OR-ed together on a framework of FPGAs. The digitized readout systems have enough memory to hold 32 bunch crossings. 


\subsubsection{The Trigger Framework}

The TFW makes the decision whether a particular event is to be accepted for further examination by using the inputs of the L1 trigger devices. The TFW uses the logical "OR" of up to 128 specific triggers conditions defined by the trigger list to determine if a given crossing holds a valid trigger. The TFW also manages the rates of triggers by applying prescale factors to keep their rates within acceptable limits. Different trigger

lists and prescale settings are passed by COOR using the trigger control computer (TCC). The TFW provides a large number of scalars to monitor trigger rates and dead times.

\subsubsection{The Level-1 Calorimeter Trigger}

The Level-1 calorimeter (L1Cal) trigger counts the number times EM and EM+HAD calorimeter towers pass a set of $E_{T}$ thresholds. L1Cal forms $\Delta \eta \times \Delta \phi=0.2 \times 0.2$ trigger towers. There are forty triggers towers in $\eta$ covering $|\eta|<4.0$. Each slice in $\eta$ has thirtytwo trigger towers covering the full $2 \pi$ of the azimuth $(\phi)$. The counts are passed to the TFW and the trigger towers are passed to the L2 calorimeter system on a $L 1$ accept.

\subsubsection{The Level-1 Central Track Trigger}

The Level-1 Central Track Trigger (L1CTT) reconstructs trajectories of charged particles using the axial hits from the CFT and the PS detectors. The CFT and CPS axial system provide triggers for charged particles using predefined track equations and matching tracks to PS clusters. The CFT and CPS axial track list is passed to L1Muon, and to the L2 tracking preprocessors on L1 accept. L1CTT conditions can be specified by the number of tracks above a $p_{T}$ threshold, with or without a PS cluster match, and track isolation. 


\subsubsection{The Level-1 Muon Trigger}

The Level-1 Muon trigger (L1Muon) uses hits from the muon wire chambers, muon scintillator counters, and tracks from L1CTT to form patterns consistent with muons. L1Muon forms trigger objects based on L1CTT tracks and muon scintillator hits. L1Muon also forms trigger objects based on matching layers of track stub (group of interlayer hits) wire hits which have been confirmed with muon scintillators. L1Muon conditions can be specified by the number of muons above a $p_{T}$ threshold, geographical region, and track quality.

\subsection{The Level-2 Trigger}

The L2 trigger system was designed to operate within a $\sim 100 \mu$ s time window and to reduce the L1 rate by a factor of 10 . During physics data taking, the L2 trigger typically receives events at a rate of $1.5 \mathrm{kHz}$ and has a rejection factor from two to five.

The L2 trigger system consists of six separate elements as shown in Figure 4.2: five individual preprocessors and one trigger decision processor, L2 Global (L2GBL). Each preprocessor generates trigger objects: muons, electrons, photons, jets, tracks, and preshower clusters, and L2GBL combines them to form the final L2 trigger decision. As an example, a L2 trigger could require one jet and one muon above a $p_{T}$ threshold. L2GBL can have up to 128 separate trigger decisions to process before accepting or rejecting an event. If the event passes at least one trigger, then this event is passed along to the L3 trigger system for further review.

\subsubsection{Level-2 Global Processor}

L2GBL receives trigger objects from the L2 preprocessors (L2 Calorimeter, L2 CTT, L2 Muon, and L2 PS). L2GBL uses the trigger list and the L1 trigger decision mask to 
decide which script to run on the objects. Each script is defined by at least one or more filters and a minimum number of objects required to pass each filter. As an example, a script could have an EM object filter with a minimum of one object. This script will pass the event if there is at least one EM object in the event that satisfies the conditions of the EM filter.

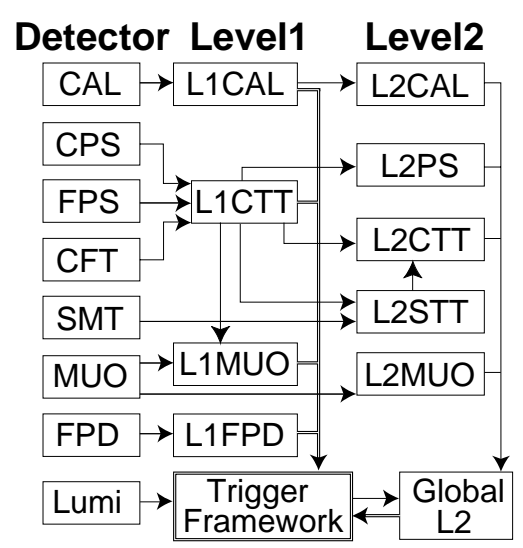

Figure 4.2: The flow of the data from the L1 inputs to the Level-2 trigger.

\subsubsection{Level-2 Calorimeter Preprocessor}

The L2 calorimeter preprocessor (L2Cal) runs EM and jet algorithms. The two algorithms receive a list of $1280 \mathrm{EM}$ and $1280 \mathrm{EM}+\mathrm{HAD}$ trigger towers from L1Cal to form the EM and jet objects. The EM algorithm forms electron and photon objects using EM trigger towers. A cluster is formed by a seed trigger tower with $E_{T}>1 \mathrm{GeV}$ and its largest $E_{T}$ neighboring EM tower. EM fraction and isolation fraction are calculated for each EM object. The jet algorithm forms jet objects by clustering $5 \times 5 \mathrm{EM}+\mathrm{HAD}$ trigger towers centered around a seed tower with $E_{T}$ greater than $2 \mathrm{GeV}$. The EM and jet objects are independently sorted in descending order of clustered $E_{T}$ and sent to L2GBL. 


\subsubsection{Level-2 Silicon Track Trigger Preprocessors}

The L2 silicon track trigger preprocessor (L2STT) uses L1CTT tracks and SMT hits to improve the momentum resolution of the tracks and to calculate track impact parameters ${ }^{1}$. The impact parameter calculation from L2STT is used to tag the decays of long-lived particles such as B hadrons. The L2STT algorithm fits track parameters by projecting L1CTT tracks into the SMT detector. The fitted tracks are sent to L2CTT.

\subsubsection{Level-2 Central Track Trigger Preprocessor}

The L2 Central Track Trigger preprocessor (L2CTT) sends tracks to L2GBL to match tracks to physics objects. L1CTT tracks are combined and sorted in descending order of $p_{T}$. The azimuthal angle, $\phi_{0}$, with respect to the beam axis is determined, as well as the azimuthal angle of the track projected to the third EM layer of the calorimeter $\phi_{e m 3}$. Several isolation criteria are calculated to enhance the trigger capabilities. Similarly in L2STT, tracks are combined and sorted, and azimuthal angles and isolation criteria are evaluated. The L2CTT processor sends three lists of tracks to L2GBL: a $p_{T}$ sorted list of L1CTT tracks, a $p_{T}$ sorted list of L2STT tracks, and an impact parameter sorted list of L2STT tracks.

\subsubsection{Level-2 Preshower Preprocessor}

The L2 Preshower preprocessor (L2PS) is used to improve the electron detection efficiency and photon separation. All three layers of the PS detectors are used to form clusters in $\eta$ and $\phi$. L2PS also reports L1CTT tracks that match with PS clusters.

\footnotetext{
${ }^{1}$ The perpendicular distance from the original center of a set of scattering particles to the original line of motion of a particle being scattered.
} 


\subsubsection{Level-1 Muon Preprocessor}

The L2 Muon preprocessor (L1Muon) combines track segments among small regions and layers of the detectors in preprocessing units called Second Level Input Computers (SLICs). The sub layers are combined into integrated muon candidates in the preprocessor. The muon candidates are sorted in descending order of $p_{T}$ and are sent to L2GBL. The quality and timing information per muon candidate are also passed to L2GBL.

\subsection{Level-3 Trigger and Data Acquisition}

The L3 trigger system is a software based trigger running a fast version of the event reconstruction algorithms in order to reduce the event rate to $50-100 \mathrm{~Hz}$. The trigger software runs on a farm of more than 100 commodity, dual $1 \mathrm{GHz}$ processors, rackmounted PCs [39] running Linux. Each detector system passes the readout information through commodity VME [40] single board computers (SBC) [41] via $100 \mathrm{MB} / \mathrm{s}$ ethernet links through a Cisco [41] 6509 switch to the farm nodes. The L3 trigger decisions are based on complete physics objects as well as the relationships between the objects. 


\section{CHAPTER 5}

\section{Offline Event Reconstruction and Object Identification}

This chapter describes how candidate electrons, jets, tracks, and vertices are reconstructed from raw detector data. A collection of complex software algorithms written in $\mathrm{C}++$ called $D \varnothing$ reco [42] is used for the reconstruction process. DØ reco unpacks the raw data, applies detector specific calibration constants, reconstructs tracks, generates a list of primary and secondary vertices, and identifies candidate objects like electrons, muons, and jets.

\subsection{Track Reconstruction}

Charged particles traversing a magnetic field leave traces along their paths when they interact with the SMT and CFT detectors. A typical event contains $10^{4}$ to $10^{6}$ hits in the central tracking system. Since charged particles can deposit energy among two adjacent silicon strips or two adjacent scintillating fibers, the hits are clustered together. The track reconstruction algorithm uses the hit clusters to find tracks. There are two track finding algorithms: Histogramming Track Finder (HTF) [43] and Alternative Algorithm (AA) [44].

The HTF method reconstructs tracks in two steps: (a) it uses a pattern recognition algorithm (histogramming) and (b) it does track fitting using a Kalman fitter [45]. In the 
$(r, \phi)$ plane the charged particles travel in circular orbits and can be uniquely defined by three parameters, $\rho, d_{0}$, and $\phi$, where $\rho=\frac{q B}{p_{T}}, \rho$ is the curvature of the track, $d_{0}$ is the distance of closest approach to the beam spot, and $\phi$ is the direction of the track at the position of closest approach to the beam spot. For every pair of hits in $(x, y)$ space there is a corresponding point (bin) in the $2 \mathrm{D}(\rho, \phi)$ space which is histogrammed. All hits forming a track have multiple pair combinations. All pair combination will have the same value (bin) in the $(\rho, \phi)$ histogram. The pattern recognition (track hypothesis) is made by taking a single hit in $(x, y)$ and extrapolating it to be a line in the $(\rho, \phi)$ histogram. All hits from the same track will have separate lines which will all intersect at the same bin, the true $(\rho, \phi)$ of the track in question.

The track list (TrackL) is passed to a 2D Kalman filter which uses $\rho, d_{0}$, and $\phi$ of each track, an expectation propagator, material effects (multiple scattering and energy loss), and the non-uniformity of the magnetic field to filter the TrackL. The remaining tracks pass through another histogramming algorithm that uses the hit locations $(r, z)$ to form lines in $\left(z_{0}, C\right)$ coordinate space. $z_{0}$ is the starting location of the track along the $z$ axis and $C=\frac{d z}{d r}$. The lines which overlap in the $\left(z_{0}, C\right)$ histogram generate a reduced TrackL. The list is processed through an $\eta$ splitter, which only allows hits moving away from the interaction point to be associated with a track when the $z$ component of the hits are increasing for $\eta>0$, and similarly when the $z$ component of the hits are decreasing for $\eta<0$.

A 3D Kalman filter is then used to build the SMT tracks and continues including hits in the CFT detector until there are too many misses in a row or the algorithm comes to the end of the detector. Beginning with the partially reconstructed track, the 3D Kalman filter extends the track by an additional measurement or hit. The track parameters and the expectation propagator are used to make an expected hit measurement. A $\chi^{2}$ value 
is calculated and if the value is within the maximum allowed limit, the hit is accepted. The track parameters are recalculated for this track, and the algorithm repeats itself by searching for the next hit. An additional HTF list is generated by starting from the CFT instead of the SMT using the same techniques. The two track lists are merged into one list and duplicate tracks are removed.

Similarly the AA uses three hits in the SMT layers for pattern recognition and then applies a track filter algorithm. For the pattern recognition, the first hit can come from any of the six layers in the silicon barrels or $\mathrm{F}$ disk. The second hit has to be within an azimuthal window, with respect to the beam spot, of less than 0.08 radians. The third hit has to be within a radius of curvature (when including the other two hits) $>30 \mathrm{~cm}$ (corresponding to a track with $p_{T} \geq 180 \mathrm{MeV}$ ), to have a track hypothesis within $2.5 \mathrm{~cm}$

of the axial impact point (at a distance of closest approach), and to have a $\chi^{2}$ value of the fit less than 16. The fitting algorithm uses the track hypothesis, which gives expectation location (search windows) for the hits at the next layers, and hits are added to the track if the $\chi^{2}$ value remains $<16$. The fitting algorithm continues until it has three consecutive misses in a row, or it reaches the end of the detector. The tracks are ordered by greatest number of hits, followed by the smallest number of misses, followed by the smallest $\chi^{2}$ value. Since the fitted tracks may share hits from other tracks, the AA requires that the number of hits shared to be less than $2 / 3$ of the total number of hits in the track. The two track lists from the HTF and AA algorithms are combined into a single list, the duplicates are removed, and the tracks are ordered in the same way as in the AA algorithm.

\subsection{Primary Vertex Reconstruction}

The primary vertices $(\mathrm{PVs})$ are the interactions points of $\bar{p} p$ collisions. The $x$ and $y$ locations of the PV fluctuates with in $40 \mu \mathrm{m}(1 \sigma)$ between events. The $z$ location of the 
PV is roughly a Gaussian distribution with a spread $(\sigma)$ of $28 \mathrm{~cm}$.

The PVs are identified in two steps [46]. First, the algorithm finds an approximate location of the beam spot and PV candidates. Second, the algorithm uses the beam spot information and tighter cuts on the tracks to identify the location of the PV. The beam spot is reconstructed using tracks with a distance of closest approach significance (dca/ $\left.\sigma_{\text {dca }}\right)$ of less than 100 with respect to the detector center in $(r, \phi)$ coordinates space. All of the tracks are used to fit the locations of the PV. The $\chi^{2}$ contribution of each track is computed with respect to this candidate. The track with the highest $\chi^{2}$ contribution is removed from the sample and the vertex is re-fitted with the remaining tracks. A new $\chi^{2}$ contribution for each track using the re-fitted vertex is computed. The process is repeated until the fitted vertex $\chi^{2}$ is less than ten. Once an approximate location of the beam spot is found, all the tracks that were not used in locating the approximate vertex location of the beam spot are used to find other vertex locations. After all of the vertices are found, the second step of the algorithm uses a new group of tracks (tighter cuts on the tracks) to fit the vertices based on their dca $/ \sigma_{\mathrm{dca}}<5$ with respect to the $(r, \phi)$ position of the vertices found in the previous step. All tracks in the final fit of the PV must have $p_{T}>0.5$ $\mathrm{GeV}$, at least two SMT hits, and dca $/ \sigma_{\mathrm{dca}}<5$. The vertices are re-fitted and the track with the largest $\chi^{2}$ contribution is removed until the vertex $\chi^{2}$ value is less than ten.

The final selection of the hard collision PV from the soft inelastic vertices comes from a $\log _{10} p_{T}$ track distribution of a Monte Carlo simulation of minimum bias events. The distribution is converted into a probability distribution [47]. A probability value for each track in each vertex is extracted from the probability distribution. For each vertex, the track probabilities are multiplied together and weighted so that the final probability value does not depend on the number of tracks associated with the vertex. The vertex with the lowest probability is considered to be the hard collision PV. The uncertainty of the $x$ and 
$y$ positions of the hard collision $\mathrm{PV}$ is approximately $6 \mu \mathrm{m}$ each.

\subsection{Electromagnetic Object Reconstruction}

The EM object reconstruction algorithm [48] generates electron and photon objects from calorimeter towers. Since photons do not leave signals in the tracking system, a track matched to the energy deposit in the calorimeter provides a tool to distinguish electrons from photons.

EM object reconstruction begins with the formation of initial calorimeter clusters. This analysis uses the simple-cone tower clustering algorithm ("Scone Method"). The simplecone algorithm clusters calorimeter towers based on precision readout data around seeds

with $E_{T}>1.5 \mathrm{GeV}$ in a cone of radius $\Delta R=\sqrt{\Delta \eta^{2}+\Delta \phi^{2}}<0.2$. all clusters satisfying the above criteria are tested for isolation:

$$
\text { Isolation }=\frac{E_{\text {total }}(\Delta R<0.4)-E_{\mathrm{EM}}(\Delta R<0.2)}{E_{\mathrm{EM}}(\Delta R<0.2)} .
$$

$E_{\text {total }}(\Delta R<0.4)$ is the total $(\mathrm{EM}+\mathrm{HAD})$ amount of energy found within a $\Delta R<0.4$. $E_{\mathrm{EM}}(\Delta R<0.2)$ is the amount of EM energy found within a $\Delta R<0.2$. All initial EM clusters are required to have an isolation of less than 0.2 . The isolation parameter gives a measure of how deep and narrow a given cluster is. EM objets tend to deposit most of their energy in a narrow region of the EM layers, while hadrons deposit their energies in the hadronic layers in a much wider radius. In addition, the isolation is used to separate the electrons produced by the $Z$ decays from the objects produced inside jets from $\pi^{0}$ decays.

A search for a track matched to the EM object is performed by projecting the $\phi$ angle and $z$ position of the track to the third EM layer of the calorimeter, and by comparing the ratio of the transverse energy of the EM object to the transverse momentum of the 
track. A $\chi^{2}$ value is calculated based on Equation 5.2:

$$
\chi^{2}=\left(\frac{\Delta \phi}{\sigma_{\Delta \phi}}\right)^{2}+\left(\frac{\Delta z}{\sigma_{\Delta z}}\right)^{2}+\left(\frac{E_{T} / p_{T}-1}{\sigma_{E_{T} / p_{T}}}\right)^{2} .
$$

$\Delta \phi$ and $\Delta z$ are the azimuthal angle and $z$ position differences between the track and the EM object at the third EM layer of the calorimeter. $E_{T} / p_{T}$ is the ratio of the transverse energy of the EM object over the transverse momentum of the track. The $E_{T} / p_{T}$ term is dropped for tracks with $\eta$ greater than 1.1 with respect to the center of the detector. If the $\chi^{2}$ probability is greater than 0.01 then the track is considered a match with the EM object. If a track match is found, the momentum is updated using the primary vertex and the location of the track is projected to the third EM layer of the calorimeter.

Electrons deposit most of their energy in the EM layers of the calorimeter and only a small fraction will leak into the fine hadronic layers. The EM fraction is defined by the following equation:

$$
\mathrm{EM} \text { fraction }=\frac{E_{\mathrm{EM}}(\mathrm{PS}+\mathrm{EM} 1+\mathrm{EM} 2+\mathrm{EM} 3+\mathrm{EM} 4)}{E_{\text {total }}(\mathrm{PS}+\text { all EM layers }+ \text { all hadronic layers })} .
$$

The shower shape of electrons differs from the shower shape of hadrons. A covariance matrix which takes into account seven discriminant variables is formed using both test beam data and MC simulated electrons. The seven variables are:

- the individual shower energy fractions found in the four calorimeter EM layers,

- the total energy of the EM cluster,

- the position of the primary vertex,

- the cluster size in the $r-\phi$ plane based on the third EM layer of the calorimeter.

The covariance matrix is defined by: 


$$
M_{i j}=\frac{1}{N} \Sigma_{n=1}^{N}\left(x_{i}^{n}-<x_{i}>\right)\left(x_{j}^{n}-<x_{j}>\right) .
$$

where $x_{i}^{n}$ is the value of variable $i$ for electron $\mathrm{n}, x_{j}^{n}$ is the value of one of the other variables $j$ for electron $\mathrm{n}$, and $\left(x_{i}\right)$ and $\left(x_{j}\right)$ are the average values for the two variables $i$ and $j$. The H-matrix is the inverse of the covariance matrix $M_{i j}$. A $\chi^{2}$ variable is calculated using the H-matrix that measures the likelihood a shower shape is consistent with an EM object shower:

$$
\chi_{k}^{2}=\Sigma_{i j}\left(x_{i}^{k}-<x_{i}>\right) H_{i j}\left(x_{j}^{k}-<x_{j}>\right) .
$$

\subsection{Jet Reconstruction and Identification}

Jets are the experimental signature of quarks and gluons. As shown in Figure 5.1, there are four jet reconstruction stages: a) parton jets, clusters of quarks and gluons produced in the hard scatter; b) particle jets, clusters of particles after the hadronization process (before hitting the detector system); c) track jets, clusters of tracks which deposited energy in the tracking system; and d) calorimeter jets, clusters of deposited energy in the calorimeter. In data, we can only measure and reconstruct track and calorimeter jets. There are two algorithms to reconstruct jets at D $\varnothing$ : the Run II mid-point jet cone

algorithm [49], [50] and the $k_{T}$ jet algorithm. The midpoint cone algorithm with a radius of $\Delta R<0.5$ was used in the analysis.

\subsubsection{The Jet Reconstruction Algorithm}

The midpoint cone algorithm reconstructs jets in the following steps:

- generates a list of seeds using preclustered calorimeter towers,

- forms proto-jets from the seed list, 
- compiles a new seed list using the midpoints between the proto-jets,

- forms proto-jets from the midpoint seed list,

- splits or merge overlapping jets,

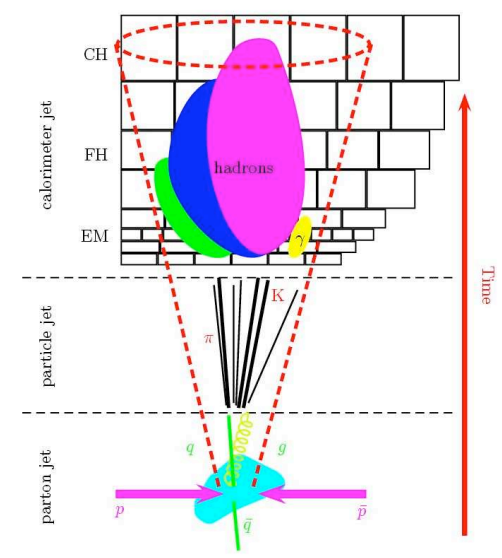

Figure 5.1: The decay paths of quarks and gluons. Quarks and gluons, after final state radiation, form parton jets. Hadrons and leptons form particle jets after hadronization. Energy deposits in the calorimeter are reconstructed as calorimeter jets.

Preclustering (seeds to the jet reconstruction algorithim): The energies of longitudinal calorimeter cells are added together to form calorimeter tower four vectors using the E-scheme:

$$
p_{\text {tower }}^{\mu}=\left(E_{\text {tower }}, p_{\text {tower }}\right)=\sum_{i=0}^{\# \text { cells in tower }}\left(E_{i}, p_{i}\right),
$$

where $p_{i}$ consists of the momentum components, $p_{x}, p_{y}$, or $p_{z}$, for each calorimeter cell. $p_{i}$ is measured with respect to the primary vertex and the center of each cell. The reconstructed towers are ordered in $p_{T}$ and all towers with $p_{T}$ greater than $0.5 \mathrm{GeV}$ are used as seeds for preclustering. If the largest cell $p_{T}$ contribution in the reconstructed tower is from the coarse hadronic region, then coarse hadronic $p_{T}$ is subtracted from the reconstructed tower before requiring the $p_{T}$ of the reconstructed tower to be $>0.5 \mathrm{GeV}$ in order to become a seed for preclustering. Starting from the first seed, the next highest 
$p_{T}$ reconstructed tower within $\Delta R=\sqrt{(\Delta \eta)^{2}+(\Delta \phi)^{2}}<0.3$ is added to the cluster and the E-scheme is used to update the center of the cluster. Once a reconstructed tower is used, it is removed from the seed list. The other reconstructed towers within $\Delta R<0.3$ are added by following the same procedure. After the clusters are formed, they are sorted in descending $p_{T}$ order, and clusters with $p_{T} \leq 1.0 \mathrm{GeV}$ are removed from the list. The calorimeter cluster list contains the seeds for the jet reconstruction algorithm.

Formation of Proto-Jets from the clustered seed list: The first seed is used to construct a proto-jet. Proto-jets have to be at least $\Delta R=\sqrt{(\Delta y)^{2}+(\Delta \phi)^{2}}>0.25$ away from each other. Rapidity is used for the rest of the cone searching algorithms, instead of pseudo-rapidity, which was used in the tower clustering algorithm. All the reconstructed towers within a cone of $\Delta R<0.5$ around the seed are added together via the E-scheme to form a proto-jet. Then the energy weighted center of the proto-jet is used to combine all the reconstructed towers within $\Delta R<0.5$. The reconstructed towers are added together via the E-scheme to make a new center. The procedure is repeated until: the $p_{T}$ of the new proto-jet is less than $4.0 \mathrm{GeV}$, the $\Delta R$ measurement refining the location of the center of the proto-jet is within 0.001 of the previous center, or the center-finding loop is repeated fifty times. The next seed from the list constructs another proto-jet. The proto-jet generating procedure repeats itself until there are no seeds left. The list of proto-jets is sorted in descending order of $p_{T}$.

Split and Merge of Overlapping Jets: The two lists of proto-jets are merged together and sorted in order of descending $p_{T}$. Starting from the jet with the largest transverse momentum, the algorithm searches for neighboring jets. If two jets are found to share reconstructed towers, and the jet with lower transverse momentum has more than half of its $p_{T}$ contribution due to the shared region, the two proto-jets are merged into one proto-jet. The proto-jet with the lower $p_{T}$ is removed and the leading- $p_{T}$ proto-jet 
is adjusted via the E-scheme using the reconstructed towers from both proto-jets. If the two proto-jets overlap and the lower $p_{T}$ proto-jet has less than half of its $p_{T}$ from the shared region, then the shared reconstructed towers are split amongst the two proto-jets. The two proto-jets divide the shared reconstructed towers based on proximity. The two proto-jets are re-constructed based on the E-scheme. The surviving proto-jets form the final list of jets. The jets are sorted in descending order of $p_{T}$ and are required to have $p_{T}>8.0 \mathrm{GeV}$.

\subsubsection{Jet Identification}

A set of quality cut variables are used for each jet to help reduce the amount of false (fake) jets found in data due to calorimeter noise. The quality cuts also help separate electron and photon objects from jets.

EM fraction: Jets deposit a large fraction of their energy in the hadronic layers of the calorimeter. Electrons and photons, on the other hand, deposit a large fraction of their energy in the EM layers of the calorimeter. The EM fraction is defined by the following equation:

$$
\mathrm{EM}_{\text {fraction }}=\frac{p_{T}^{\mathrm{EM}}}{p_{T}^{\text {total }}}
$$

where $p_{T}^{\mathrm{EM}}$ is the amount of transverse momentum in the EM layers of the calorimeter for a given jet. $p_{T}^{\text {total }}$ is the total amount of transverse momentum of the jet.

Coarse hadronic fraction (CHF): The noisiest part of the calorimeter is the coarse hadronic section. The coarse hadronic fraction is defined as the amount of transverse momentum deposited in the coarse hadronic layers divided by the total amount of transverse momentum in the jet. 


$$
\mathrm{CHF}=\frac{p_{T}^{\text {coarse hadronic layer }}}{p_{T}^{\text {total }}} .
$$

Hot Cell Fraction (HOTF): HOTF is defined as the ratio of the highest $p_{T}$ cell divided by the next highest $p_{T}$ cell in the jet. Jets with noisy calorimeter cells are removed with this cut.

n90: A jet deposits its energy over a series of calorimeter towers. Electrons/photons and single noisy (hot) towers will deposit most of their energy in one tower. N90 is the number of towers in the jet which make up $90 \%$ of the transverse momentum of the jet.

Level-1 Confirmation: To further reduce spurious jets coming from precision readout noise problems, the jet $p_{T}$ is compared to the energy found by the L1 trigger system. L1 confirmation is defined by:

$$
\mathrm{L} 1 \text { confirm }=\frac{\mathrm{L} 1 \text { set }}{p_{T}^{\text {reco×(1-CHF })}},
$$

where L1set is the summation of the transverse energy of the Level-1 trigger towers inside the jet. $p_{T}^{\text {reco }}$ is the uncorrected jet transverse momentum (before Jet Energy Scale corrections).

\subsection{Muon Reconstruction}

Muons produced in semi-leptonic decay of B hadrons are used in this analysis as as additional constraint when tagging b-quark initiated jets. The muons are reconstructed [51] by the muon detectors using hit information from the A and BC layers. Hits in these layers form segments. A muon is identified when it has at least two wire hits and one scintillator hit in the A layer, and at least two wire hits and one scintillator hit in the BC layers. The momentum of the muon is calculated by combining segments from the $\mathrm{A}$ region and $\mathrm{BC}$ layers using the curvature of the muon paths. Once a muon is 
identified, a search in $(\Delta \eta, \Delta \phi)$ of the projected location of the muon is conducted in the tracking system. If multiple tracks are found, the track with the lowest $\chi_{\text {track }}^{2}$ value is used as the track match. The momentum vector of the muon is recalculated based on the momentum vector of the track. Cosmic ray muons are rejected using timing information from the scintillator hits. The muons used in this analysis have $p_{T}>15.0 \mathrm{GeV}$, IP (impact parameter) along the $z$ axis $<1 \mathrm{~cm}$ from the primary vertex, and a match to a jet within $\Delta R_{\text {muon, jet }}<0.5$. 


\section{CHAPTER 6}

\section{Data Samples and Luminosity}

This analysis uses data that were collected in the 2002-2006 physics runs. The raw data were reconstructed using the RECO versions of p17.09.03 and p17.09.06. The data were further processed by D0CORRECT to correct EM, MUON, JET and $\mathscr{E}_{T}$ objects. The resulting data were skimmed according to selection criteria set by the $\mathrm{D} \varnothing \mathrm{Common}$ Sample (CS) group [52] and put into SAM. The details can be found in the CS group web site. This analysis uses two data sets that are split into three skims:

- CSG_CAF_2EMhighpt_PASS3_p17.09.03

- CSG_CAF_2EMhighpt_PASS3_p17.09.06

- CSG_CAF_2EMhighpt_PASS3_p17.09.06b

- CSG_CAF_2MUhighpt_PASS3_p17.09.03

- CSG_CAF_2MUhighpt_PASS3_p17.09.06

- CSG_CAF_2MUhighpt_PASS3_p17.09.06b

The selection requirements of the 2EMhighpt skim are as follows:

- EM $|I D|=10,11$ and $p_{T}>12.0 \mathrm{GeV} / c$ for the first EM object.

- EM $|I D|=10,11$ and $p_{T}>12.0 \mathrm{GeV} / c$ for the second EM object. 
- The EM objects are reconstructed using the simple cone algorithm.

The skim definition for the 2MUhighpt skims are as follows:

- The first muon is Loose and has a central track with $p_{T}>10.0 \mathrm{GeV} / \mathrm{c}$.

- The second muon is Loose and has a central track with $p_{T}>10.0 \mathrm{GeV} / \mathrm{c}$.

These data sets were then turned into CAF trees with the p18.05.00 release. The 2EMhighpt data are used for the eee and ee $\mu$ final state event selections, while the 2MUhighpt data are used for the $\mu \mu \mu$ and $\mu \mu$ final state event selections. The skims:

- CSG_QCD_PASS3_p17.09.03

- CSG_QCD_PASS2_p17.09.01

- CSG_EMMU_PASS3_p17.09.03

- CSG_EMMU_PASS2_p17.09.06

- CSG_EMMU_PASS3_p17.09.06b

are also used for background study purposes. These skims were skimmed with the following requirements:

- The QCD Skim requirements used the following triggers:

- JT_125TT
- JT_95TT
- JT_65TT
- CJT5
- JT_8TT
- JT_15TT
- JT_25TT_NG


- JT_45TT

- JT_L3M225

- JT_L3M250

- JT_L3M380

- JT_L3M430

- The EMMU skim requirements are:

- An EM object with $p_{T}>5.0 \mathrm{GeV} / \mathrm{c}$ and $\mathrm{EM}|I D|=10,11$

- A Loose muon with a central track with $p_{T}>10.0 \mathrm{GeV} / \mathrm{c}$.

- The EM objects are reconstructed using the simple cone algorithm.

The following data quality requirements are applied to the EM data:

- CAL: "unknown" or better

- SMT: "reasonable"

- CFT: "good" or "reasonable"

The following data quality requirements are applied to the muon data:

- CAL: "unknown" or better

- SMT: "reasonable"

- CFT: "good" or "reasonable"

- MUON: "good" or "reasonable"

Events with bad luminosity blocks were also rejected as prescribed by the data quality groups [53]. To properly normalize the data samples we must calculate the luminosity in which the on-line triggers that collect our signature were fired. The triggers that this analysis takes advantage of are single and di-electromagnetic object (EM) triggers that 
trigger only on calorimeter objects and the single muon triggers. The trigger versions and

the triggers used in the version are listed in Section D. These trigger lists are used in the

event selection, efficiency calculation and background estimation.

Table 6.1: This table shows the integrated luminosity for the 2EMhighpt data sample. The run ranges and integrated luminosity for the major trigger versions are shown. Along with the current luminosity is listed the luminosity that is used for the preliminary measurement as well as the ratio of the two luminosity constants. The data that did not need re-fixing is listed under the v14(b) data epoch. The cable swap data, v14(c), is included but was not included until after the new luminosity constant and therefore does not have an old luminosity for comparison. The total numbers are for the old luminosity (up to v14(b)) and all of the new luminosity. All of the luminosity numbers have an associated $6.5 \%$ uncertainty.

\begin{tabular}{|c|c|c|c|c|}
\hline $\begin{array}{c}\text { Trigger } \\
\text { Version }\end{array}$ & Run Ranges & $\begin{array}{c}\text { EM Trigger } \\
\text { Luminosity }\left(\mathrm{pb}^{-1}\right)\end{array}$ & $\begin{array}{c}\text { New Luminosity } \\
\text { Constant }\left(\mathrm{pb}^{-1}\right)\end{array}$ & Ratio \\
\hline \hline v8-v11 & $160582-178721$ & 102 & 118 & 1.16 \\
v12 & $178722-194566$ & 206 & 239 & 1.16 \\
v13.0 & $194567-195838$ & 18 & 35 & 1.93 \\
v13.2 & $195839-208203$ & 303 & 340 & 1.12 \\
v14(a) & $208204-213063$ & 127 & 140 & 1.11 \\
v14(b) & $213064-214999$ & 89 & 100 & 1.11 \\
v14(c) & $215000-215670$ & $\mathrm{n} / \mathrm{a}$ & 97 & $\mathrm{n} / \mathrm{a}$ \\
\hline \hline Total & $160582-215670$ & 845 & 1068 & 1.15 \\
\hline
\end{tabular}

Table 6.2: This table shows the integrated luminosity for the 2EMhighpt data sample with muon quality cuts applied. The run ranges and integrated luminosity for the major trigger versions are shown. Along with the current luminosity is listed the luminosity that is used for the preliminary measurement as well as the ratio of the two luminosity constants. The data that did not need re-fixing is listed under the v14(b) data epoch. The cable swap data, v14(c), is included but was not included until after the new luminosity constant and therefore does not have an old luminosity for comparison. The total numbers are for the old luminosity (up to v14(b)) and all of the new luminosity. The ratio is calculated before applying the muon quality cuts. All of the luminosity numbers have an associated $6.5 \%$ uncertainty.

\begin{tabular}{|c|c|c|c|c|}
\hline $\begin{array}{c}\text { Trigger } \\
\text { Version }\end{array}$ & Run Ranges & $\begin{array}{c}\text { EM Trigger } \\
\text { Luminosity }\left(\mathrm{pb}^{-1}\right)\end{array}$ & $\begin{array}{c}\text { New Luminosity } \\
\text { Constant }\left(\mathrm{pb}^{-1}\right)\end{array}$ & Ratio \\
\hline \hline v8-v11 & $160582-178721$ & 92 & 107 & 1.16 \\
v12 & $178722-194566$ & 200 & 231 & 1.16 \\
v13.0 & $194567-195838$ & 20 & 32 & 1.60 \\
v13.2 & $195839-208203$ & 300 & 336 & 1.12 \\
v14(a) & $208204-213063$ & 123 & 136 & 1.11 \\
v14(b) & $213064-214999$ & 89 & 99 & 1.11 \\
v14(c) & $215000-215670$ & $\mathrm{n} / \mathrm{a}$ & 95 & $\mathrm{n} / \mathrm{a}$ \\
\hline \hline Total & $160582-215670$ & 836 & 1023 & 1.15 \\
\hline
\end{tabular}

Tables 6.1, 6.2, and 6.3 list the integrated luminosity of the data sets used in the 
Table 6.3: This table shows the integrated luminosity for the 2MUhighpt data sample. The run ranges and integrated luminosity for the major trigger versions are shown. Along with the current luminosity is listed the luminosity that is used for the preliminary measurement as well as the ratio of the two luminosity constants. The data that did not need re-fixing is listed under the v14(b) data epoch. The cable swap data, v14(c), is included but was not included until after the new luminosity constant and therefore does not have an old luminosity for comparison. The total numbers are for the old luminosity (up to v14(b)) and all of the new luminosity. All of the luminosity numbers have an associated $6.5 \%$ uncertainty.

\begin{tabular}{|c|c|c|c|c|}
\hline $\begin{array}{c}\text { Trigger } \\
\text { Version }\end{array}$ & Run Ranges & $\begin{array}{c}\text { Muon Trigger } \\
\text { Luminosity }\left(\mathrm{pb}^{-1}\right)\end{array}$ & $\begin{array}{c}\text { New Luminosity } \\
\text { Constant }\left(\mathrm{pb}^{-1}\right)\end{array}$ & Ratio \\
\hline \hline v8-v11 & $160224-177283$ & 45 & 52 & 1.16 \\
v12 & $177284-194566$ & 213 & 248 & 1.16 \\
v13-v14(a) & $194567-213063$ & 420 & 467 & 1.11 \\
v14(b) & $213064-214999$ & 83 & 92 & 1.11 \\
v14(c) & $215000-215670$ & n/a & 85 & n/a \\
\hline \hline Total & $160224-215670$ & 761 & 944 & 1.13 \\
\hline
\end{tabular}

$W Z$ production search. A standard $6.5 \%$ uncertainty is associated with the luminosity normalization procedure. For trigger version 14 there are two separate data sets, the re-fixed set (a) and the set that did not need to be re-fixed (b). The reconstructed "cable swap" data is contained in the epoch listed for trigger version 14(c).

The total integrated luminosity for the data sample skims is $944 \mathrm{pb}^{-1}$ for the $2 \mathrm{MUhighpt}$ skims and $1.07 \mathrm{fb}^{-1}$ for the 2EMhighpt skims, when the muon data quality is applied to the 2EMhighpt skim the luminosity for that skim then becomes $1.02 \mathrm{fb}^{-1}$. The reason for calculating the luminosity again with muon quality applied to the 2EMhighpt is for properly normalizing the data set for the ee $\mu$ signal channel. 


\section{CHAPTER 7}

\section{Data Analysis}

\subsubsection{Lepton Identification}

Development of the Common Analysis Format (CAF) and the Common Analysis Format Environment (CAFE) [54] has centralized physics object identification and allowed for analysis to become more efficient. This analysis takes advantage of this commonality. The electron identification (ID) uses the Loose_trk ID version 2 [55]. The muon identification uses the Loose, trkloose ID version 1 [56] with additional isolation requirements.

\section{Electron Identification}

To summarize the electron identification for Loose_trk version 2, the following cuts are applied to EM objects:

- $\mathrm{EM}|I D|=10,11$

- good calorimeter isolation of the EM cluster:

$$
f_{\text {iso }} \equiv \frac{E_{\text {tot }}(0.4)-E_{\text {em }}(0.2)}{E_{\text {em }}(0.2)}<0.2
$$

- high EM fraction of the calorimeter cluster:

$$
f_{\text {em }} \equiv E_{\text {em }} / E_{\text {tot }}>0.9
$$

- $E_{T}>15 \mathrm{GeV} / c^{2}$ 
- electron likelihood $>0.2$

The likelihood is a $\chi^{2}$ comparison that uses several EM object properties, two of which are the electron shower shape and the object's track match probability. The electron detector $\eta$ coverage is limited to be within $|\eta|<1.1$ or $1.5<|\eta|<2.5$. Conventionally, in the $\mathrm{D} \varnothing$ experiment, the electrons in $|\eta|<1.1$ and with $0.1<\bmod \left(\phi_{\text {cluster }}^{e}, 2 \pi / 32\right)<0.9$ (the selected electrons cannot be within $10 \%$ of a calorimeter module edge) are called CC electrons and the electrons in $1.5<|\eta|<2.5$ are called EC electrons. Most analysis, such as $Z \rightarrow e e$ and $W \rightarrow e \nu$ cross section measurements, use only CC and EC electrons defined as above. To increase the event acceptance, the electrons in the edge of CC modules, which are called CC-edge electrons, are considered in this analysis.

\section{Muon Identification}

Muon identification criteria optimized for high muon efficiency are used in this analysis. The requirements from which the muon identification is determined are the quality of the muon object, the number of muon chamber track segments (or Nsegs), if the cosmic veto cut is required and also the distance of closest approach (or dca). All of these variables are explained in the muon ID note [51], but they are summarized here for the reader's convenience. The muon object quality, Loose, is dependent on the number of wire and scintillator hits a track has within the muon system. The quality is also dependent on the Nseg parameter. A positive $N$ seg is a muon reconstructed in the muon system with a matched track from the central tracking system. The value of the $N$ seg can be $0, \pm 1, \pm 2, \pm 3$. These values correspond to the type of track segments reconstructed in the muon system. The cosmic veto is a flag that depends on the difference in time of the scintillator hits of the track with the event bunch crossing. If the absolute value of the scintillator time, $t$, is greater than $10 \mathrm{~ns}$ then the muon is considered to be a cosmic 
muon. The $d c a$ is the impact parameter of the muon track. It is the shortest distance from the primary vertex to the track measured in the $x-y$ plane. The muon object is required to pass the following cuts:

- Muon object quality of Loose

- $N \operatorname{seg}>0$.

- cosmic veto.

- spatial track match.

- The $d c a$ of the muon track in the transverse plane compared to the primary vertex should be less than $0.02 \mathrm{~mm}$ if the track includes a hit in the silicon micro-strip tracker (SMT).

- The $d c a$ should be less than $0.2 \mathrm{~mm}$ if the track does not have a hit in the SMT.

Finally we apply a set of isolation requirements to our muons. A muon is considered isolated in the tracker if the $p_{T}$ sum of all of the tracks that share the same vertex as the muon, in a cone of radius $(\mathcal{R})$ of $0.5^{1}$, is less than $3.5 \mathrm{GeV}$. A muon is considered isolated in the calorimeter if the energy sum of all the cells, in a hollow cone of outer radius $(\mathcal{R}) 0.4$ and inner radius $(\mathcal{R})$ of 0.2 , is less than $2.5 \mathrm{GeV}$. The efficiencies for these isolation requirements were determined using the standard procedure outlined in the muon ID certification note [51]. These efficiencies were then translated into a text file (.spc) to be used in the CAFE framework as described in the muo_cert web page [57 ${ }^{2}$. These isolation requirements, optimized in the previous $W Z$ search, help reduce the QCD source of background [17].

\footnotetext{
${ }^{1} \mathcal{R}=\sqrt{\Delta \eta^{2}+\Delta \phi^{2}}$, where $\Delta \eta$ and $\Delta \phi$ are the difference in $\eta$ and $\phi$ of the muon track and another track.

${ }^{2} \mathrm{~A}$.spc file is applied to MC to correct detector level object efficiencies.
} 


\subsubsection{Vector Boson Selection Criteria $Z$ selection}

The selection of $Z$ bosons is done using the WZ_CAFRECO v2.3 [58] package. This package selects $Z$ candidates using leptons matching our lepton quality cuts. A $Z$ candidate is considered any pair of good leptons that reconstruct an invariant mass falling within a mass window that is five times the width of the $Z$ mass peak centered about 91.188 $\mathrm{GeV} / \mathrm{c}^{2}$. The mass window differs depending on the lepton family. In the case where more than two leptons from the same family are in an event then the pair that reconstructs the invariant mass closest to the $Z$ mass peak is determined to be that of the $Z$ boson. This method of selecting the pair of leptons from the $Z$ decay when more than two leptons of the same family are present introduces a combinatorics issue when the possibility that Drell-Yan $+W$ events are considered. Investigations of this effect demonstrate that we expect $1 \%$ of our eee events and $1.4 \%$ of our $\mu \mu \mu$ events to be from this source. Further requirements of $Z$ boson selection are dependent on the family of the daughter leptons.

\section{$Z \rightarrow e e$ selection}

Selection of $Z \rightarrow e e$ events is done by selecting two good electrons that meet the electron identification criteria outlined in section 7.0.1. The invariant mass window cut for electron pairs is $71<M_{Z}<111 \mathrm{GeV} / \mathrm{c}^{2}$. No further requirements are imposed on the $Z \rightarrow e e$ pair at this point in the selection. The dielectron invariant mass distribution is shown in Figure 7.1.

\section{$Z \rightarrow \mu^{+} \mu^{-}$selection}

Selection of $Z \rightarrow \mu^{+} \mu^{-}$events are done by first selecting two good muons that meet the muon identification criteria described in Section 7.0.1. Next the muons are required to be of opposite charge. The efficiency for requiring opposite charges of dimuons is 


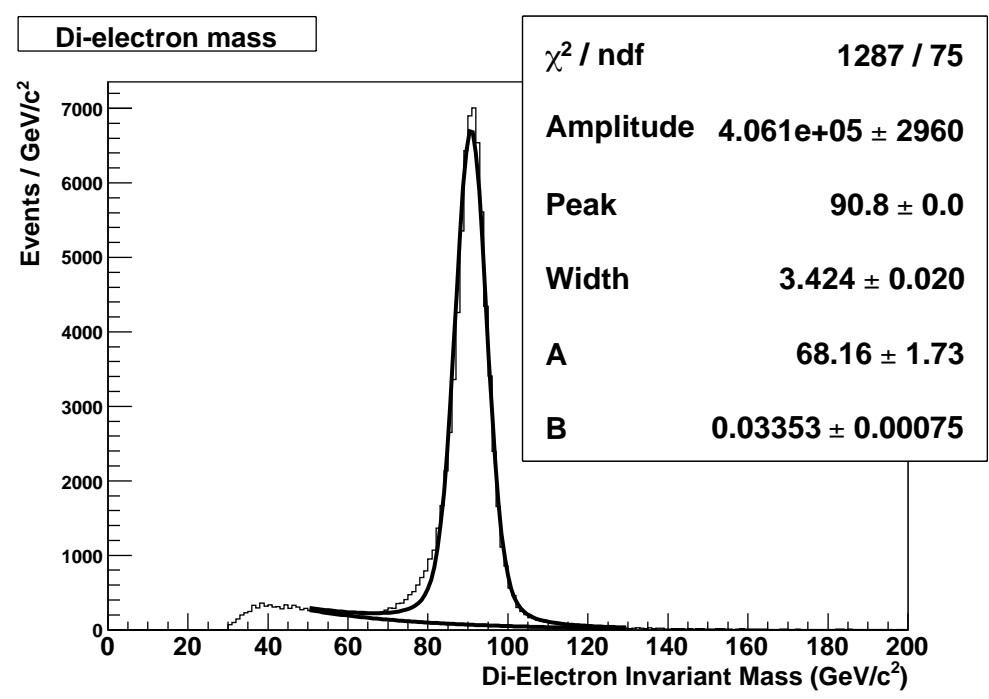

Figure 7.1: The ee invariant mass distribution of the signal data sample before the analysis mass cuts are applied. The fits used are a Gaussian convoluted with a Lorentzian which are plotted on top of an exponential fit. The exponential fit is to model the background.

$0.997 \pm 0.03$ [59]. Third, the muon pairs must have an acolinearity $(\mathcal{A})^{3}$ greater than 0.05 radians. This cut is designed to reduce cosmic backgrounds. The $Z$ mass window for a pair of muons is $50<M_{Z}<130 \mathrm{GeV} / \mathrm{c}^{2}$. No further requirements are imposed on the $Z \rightarrow \mu^{+} \mu^{-}$pair at this point in the selection. The dimuon invariant mass distribution is shown in Figure 7.2.

\section{$W$ boson selection}

After a $Z$ boson candidate has been selected, the signature of a $W$ boson is searched for in the event. First, we demand that at least one more lepton that meets our lepton identification criteria, as described in section 7.0.1, must be present in the event. Next, we require that the $\mathbb{E}_{T}$ in the event must be greater than $20 \mathrm{GeV}$. The $\mathbb{E}_{T}$ is the imbalance of transverse energy $\left(E_{T}\right)$ measured in the calorimeter. A large amount of $E_{T}$, such as $20 \mathrm{GeV}$, is an indication that a neutrino is present in the event. The $\mathbb{E}_{T}$ is corrected for any good muons in the event, as muons tend to leave little energy in the calorimeter.

\footnotetext{
${ }^{3}$ Acolinearity is the difference of the two muon track from a straight line, i.e. $\mathcal{A} \equiv|(\Delta \phi+\Delta \theta)-2 \pi|$.
} 


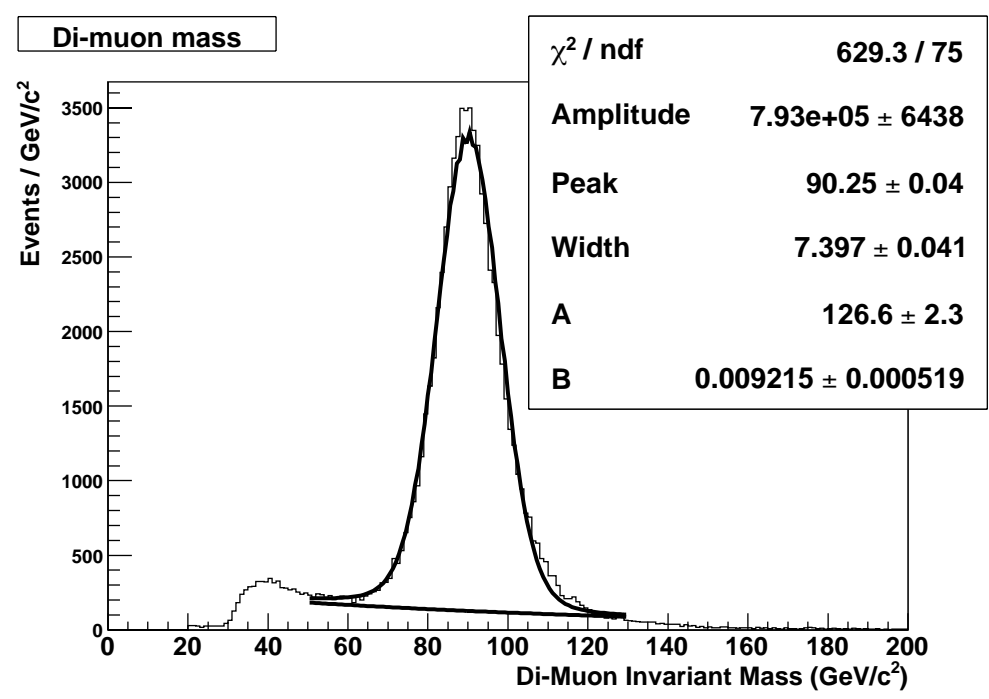

Figure 7.2: The $\mu \mu$ invariant mass distribution in the signal data sample before the analysis mass cuts are applied. The fits used are a Gaussian convoluted with a Lorentzian which are plotted on top of an exponential fit. The exponential fit is to model the background.

\subsubsection{Additional Signal Criteria}

After we have found the signatures of both a $Z$ and $W$, we require that the leptons all be separated from each other by a radius $(\Delta \mathcal{R})$ of 0.2 . The radius of separation is calculated from the $\eta$ and $\phi$ of the leptons by the equation

$$
\Delta \mathcal{R}=\sqrt{\left|\eta_{\ell}-\eta_{\ell^{\prime}}\right|^{2}+\left|\phi_{\ell}-\phi_{\ell^{\prime}}\right|^{2}} .
$$

The track $z$ 's of all the leptons must come from within a $3 \mathrm{~cm}$ window of each other. This $\Delta z_{\text {tracks }}$ is determined by the equation

$$
\Delta z_{\text {tracks }}=\left|z_{\ell}-z_{\ell^{\prime}}\right| .
$$

Finally a cut is performed to reduce the $t \bar{t}$ background contribution. This is done by taking advantage of the differences of hadronic energy produced when $W Z \rightarrow \ell \ell \ell^{\prime}$ events and $t \bar{t}$ dilepton events are compared. We calculate the hadronic energy using the formula 


$$
V E_{T H a d}=\left|\sum_{\text {leptons }} \overrightarrow{E_{T \ell}}+\overrightarrow{E_{T}}\right|
$$

where $\sum_{\text {leptons }} \overrightarrow{E_{T \ell}}$ is the vector sum of the $E_{T}$ of the charged leptons in the event and $\overrightarrow{E_{T}}$ is the transverse vector of the missing $E_{T}$. The $V E_{T H a d}$ is balanced by the hadronic part of the event. We reject any events with $V E_{\text {THad }}>50.0 \mathrm{GeV}$.

The final $W Z$ event selection is summarized here:

- Total number of leptons (electrons or muons) $\geq 3$.

- $E_{T}>20 \mathrm{GeV}$.

- Separation between any two leptons must be $|\Delta \mathcal{R}|>0.2$

- All leptons come from the same vertex: $\left|\Delta z_{\ell \ell}\right|<3.0 \mathrm{~cm}$.

- $V E_{T H a d}<50 \mathrm{GeV}$

After applying the above selection criteria to the data set, we find 13 candidate events. The candidates event views are given in the appendix, Section E.

After applying all of the cuts, two events are found in each of the eee and $\mu \mu \mu$ channels, one event is found in the ee $\mu$ channel, and eight events are found in the $\mu \mu e$ channel. These 13 events are well within agreement with our expected signal plus background estimate. A summary of the observed $W Z$ events is listed in the appendix Section E. Shown in Figure 7.3 is the invariant mass of the candidates. In Figure 7.4 is the transverse mass of the candidates. In Figure 7.5 is the plot of dilepton invariant mass versus $\mathbb{E}_{T}$.

A probability study was performed to help quantify the probability of getting this distribution of events across the four channels. Ten million trials were performed where the number of events in each channel were allowed to fluctuate according to Poisson statistics that is convoluted with Gaussian errors taken from the uncertainties on the MC expected events and the estimated backgrounds per channel. Of the ten million trials, 
4904207 trials had 13 or more events. Of the 4904207 trials, 711238 had a single channel with 8 or more events. This is a $15 \%$ probability. Of the ten million trials, 1075908 had exactly 13 candidates. Of those 1075908 trials, 32630 had a single channel with 8 or more events. This is a $3 \%$ probability. Of the 1075908 trials with exactly 13 candidates, 26077 had a single channel with exactly 8 events. This is a $2.4 \%$ probability. Of the 1075908 trials with exactly 13 candidates, 8657 had exactly 8 events in the particular channel that this analysis finds. This is a $0.8 \%$ probability.

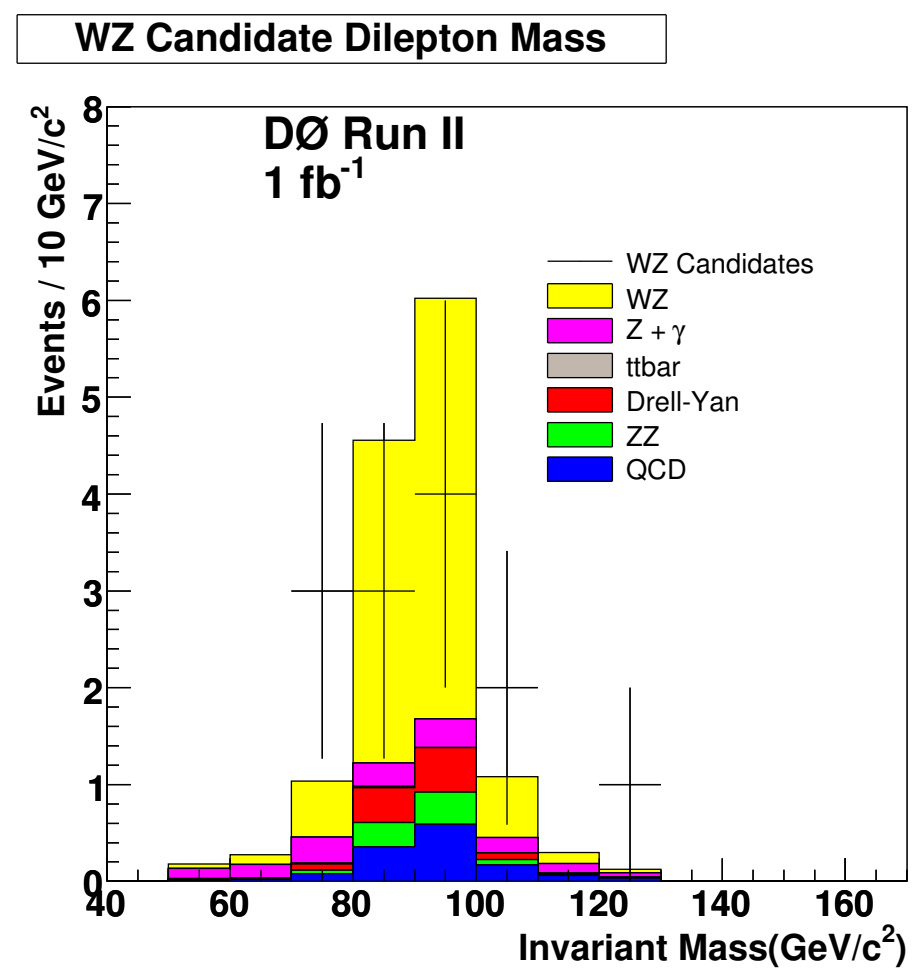

Figure 7.3: Shown is the dilepton mass of the $Z$ boson pair from each candidate event along with the background and MC signal overlaid.

\section{$7.1 \quad$ Efficiency}

In this analysis there are three main efficiencies. One is the geometric and kinematic acceptances $(\mathcal{A})$ of the signal event. These acceptances are estimated by using signal MC and are determined for each event type separately. A second efficiency is the lepton 


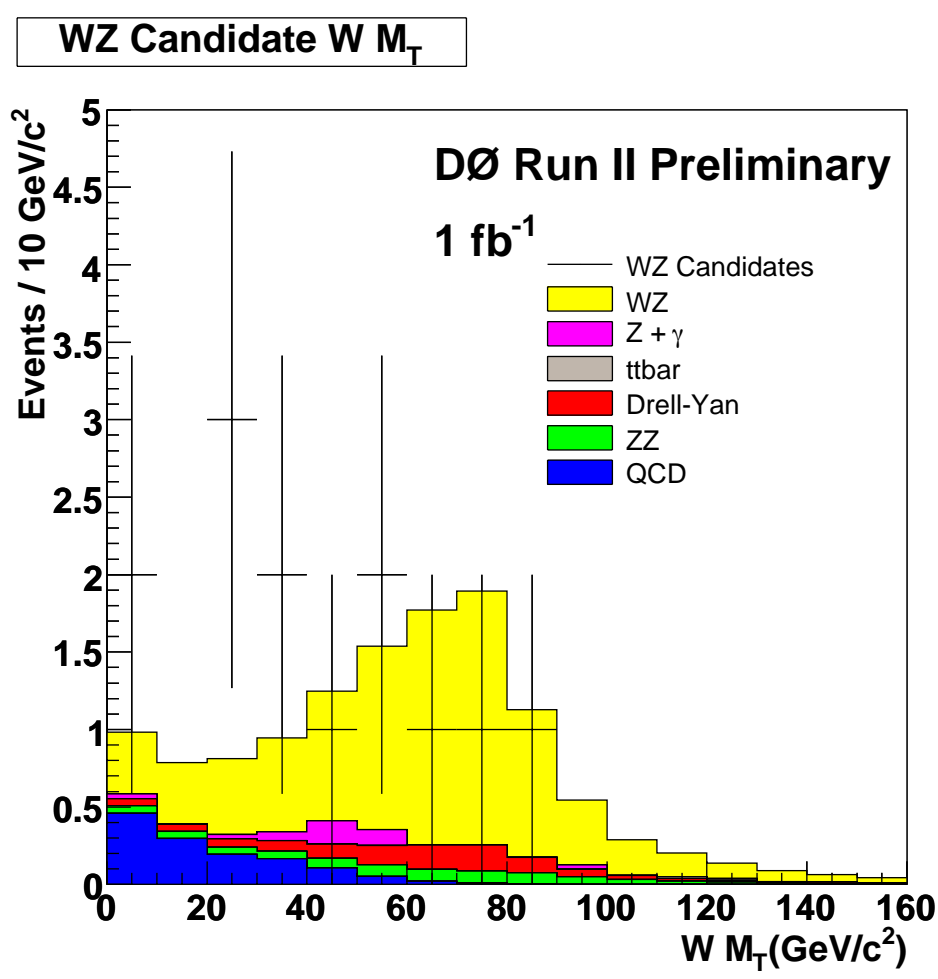

Figure 7.4: Shown is the distribution of the transverse mass of the third lepton and $\mathbb{E}_{T}$ along with the background and MC signal overlaid.

ID efficiency $(\epsilon)$. This is the efficiency at which the detector will properly identify a lepton. The lepton ID efficiencies are determined on a lepton by lepton basis and are folded with the acceptance. The third main efficiency is the trigger efficiency. The trigger efficiencies depend on the channel and are therefore applied on a per channel basis. The identification efficiencies are determined by lepton ID groups and then implemented into CAFE. To determine the full efficiency for a signal channel, we apply a correction factor, or weight, to the $\mathrm{MC}$ event. These correction factors have been determined by comparing lepton identification in the data to the MC $[60,51]$. In the case of this analysis there are three leptons per event. Each lepton will have a different weight depending on its kinematics. So the event weight is a product of all the efficiency weights in the event. The final acceptance times efficiency is then determined by the following formula, 


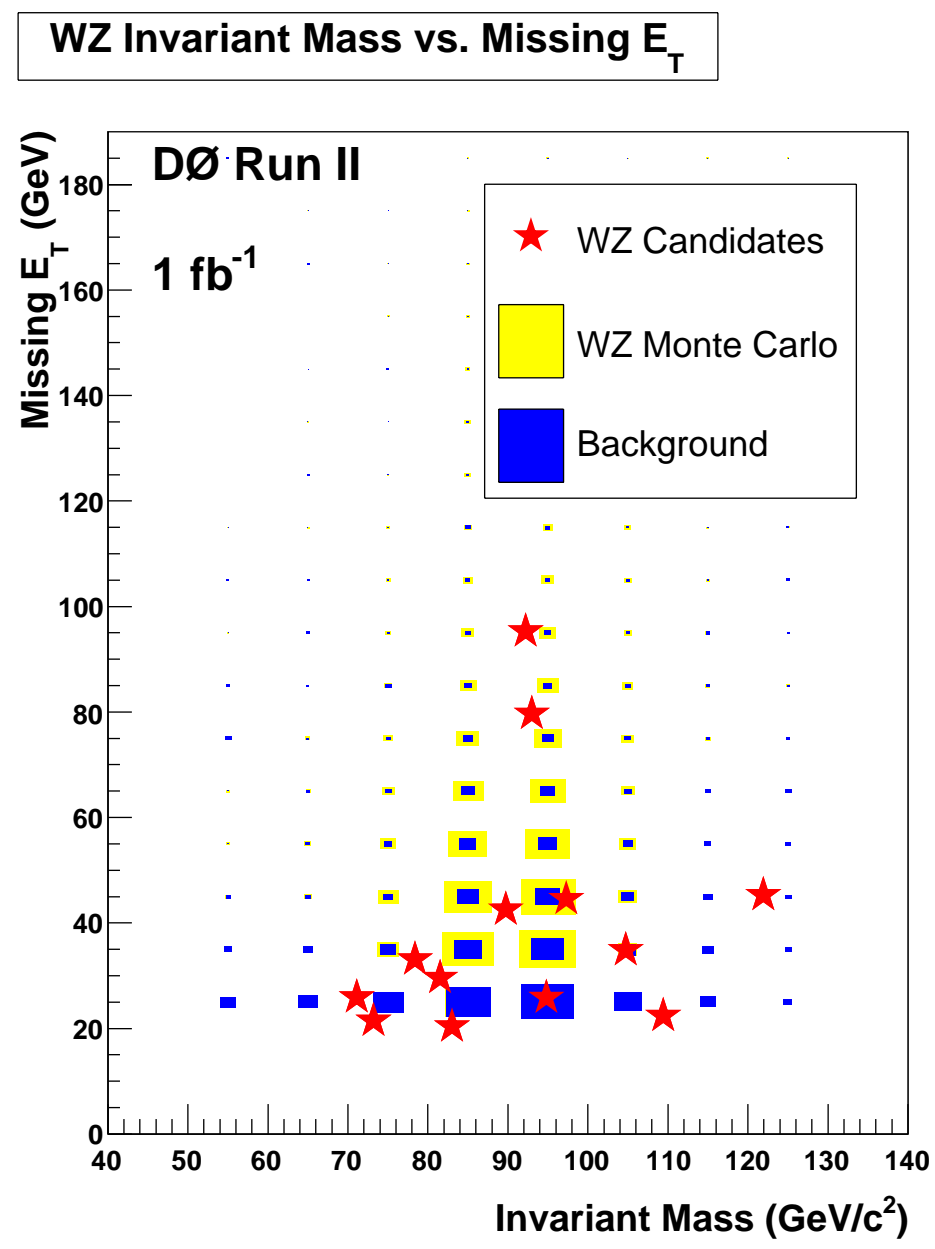

Figure 7.5: Shown is the dilepton invariant mass versus $\mathbb{E}_{T}$ of the candidate events along with the sum of the background estimates and expected signal. The MC are normalized to the data luminosity.

$$
\mathcal{A}_{i} \times \epsilon_{i}=\mathcal{A}_{i} \times \epsilon_{\ell_{1}} \times \epsilon_{\ell_{2}} \times \epsilon_{\ell_{3}} \times \epsilon_{\text {trigger }}
$$

and is determined for each channel. The $\mathcal{A}_{i}$ is folded into the efficiency on an event by event basis and is dependent on the individual event kinematics. It is estimated by the acceptance study described in the next section.

\subsubsection{Acceptance}

Acceptance of our signal is determined by applying all of our kinematic selection cuts to a sample of signal events generated from PyтнiA Monte Carlo (MC). The MC events 
are passed through the full detector simulation, using GEANT3 and then finally passed through the full data reconstruction algorithm, p17.09.01. The request ID numbers for the $W Z$ signal MC are 26148, 26149, 26150. The generated MC samples are $W Z$ to trilepton events including tau decay channels, where the $Z$ is produced on mass shell. This analysis does not limit itself to only the muon and electron daughters of $W Z$ decays, but it only identifies muons and electrons in the final state. We consider only the leptonic decay of taus from the $W Z$ decay as part of our final signal acceptance. The MC samples were further skimmed into the four different signal channels and stored on a local disk. The total number of eee, ee $\mu, \mu \mu e$ and $\mu \mu \mu$ events are 23116, 23038, 22954 and 23088 respectively. In addition there is also a total of 6489 signal events that include a $\tau$ intermediate state. For this acceptance study, though, only the direct decays to electrons and muons are considered.

From these MC samples, events are selected in the same fashion as the real data samples, but we do not require lepton ID's, except that an electron is required to have $\mathrm{EM}|I D|=10,11$. Specifically, at least three reconstructed leptons and $\mathscr{E}_{T}>20 \mathrm{GeV}$ are required in an event. The electrons must be in the fiducial region: $|\eta|<1.1$ or $1.5<$ $|\eta|<2.5$ and have a $p_{T}>15.0 \mathrm{GeV}$. The $p_{T}$ of the muons must be larger than $15.0 \mathrm{GeV}$. The separation between any pair of leptons must be larger than 0.2 . The results of the acceptance studies are shown in Table 7.1.

Table 7.1: Acceptance of $W Z$ diboson events for different trilepton decay channels. The acceptance is calculated based on the kinematic and the geometric cuts. The uncertainties on the acceptances are purely statistical.

\begin{tabular}{|c|c|c|c|c|}
\hline \hline Decay Channel & $e e e$ & $e e \mu$ & $\mu \mu e$ & $\mu \mu \mu$ \\
\hline Generated & 23116 & 23038 & 22954 & 23088 \\
Candidates & 6709 & 8221 & 8915 & 10314 \\
\hline Acceptance & 0.290 & 0.357 & 0.388 & 0.447 \\
\hline Uncertainty & 0.003 & 0.003 & 0.003 & 0.003 \\
\hline \hline
\end{tabular}




\subsubsection{Electron Efficiency}

The electron ID requirements are summarized in Section 7.0.1. The efficiencies for a single electron with these requirements to be detected by the D $\varnothing$ detector are determined by the EM ID group [60]. The group determines the efficiencies by using a tag-andprobe method from a pure sample of $Z \rightarrow e e$ events. The group also performs the same study on electrons from $Z \rightarrow e e$ PYTHIA generated MC. The group then calculates the correction factor that is needed to correct the MC so that it resembles real data. The efficiency is roughly $90 \%$ in the central calorimeter and more like $50 \%$ in the forward calorimeters, although it has strong dependence on $|\eta|$ which is accounted for in the MC. The correction factor from data to $\mathrm{MC}$ is greater than 0.9 for $|\eta|<2$, but drops at higher $|\eta|$ These correction factors are applied in our signal MC so that we may determine the full acceptance times efficiency of our analysis ${ }^{4}$.

The electron trigger efficiency for our $Z \rightarrow$ ee sample is considered to be $99 \pm 1 \%$ efficient as prescribed by the trigger study group[61].

\subsubsection{Muon Efficiency}

Certified muon efficiencies are determined by a tag-and-probe method applied to a sample of pure $Z \rightarrow \mu \mu$ events [51]. The muon ID efficiency and track match efficiency are provided in the same manner as the electron efficiencies. Calculation of the muon efficiency corrections to the MC, as performed in the CAFE framework, are done on the fly. In other words there is no correction file provided a priori, only an efficiency file for data and one for MC. The efficiency for this is usually greater than $90 \%$, but depends on eta, phi and the $\mathrm{z}$ position of the interaction. All of these effects are accounted for by the MC.

\footnotetext{
4 The uncertainty on the efficiencies are estimated by applying a conservative $2 \%$ relative uncertainty to the MC correction weights. This is applied in CAFE by varying the correction weights by $\pm 2 \%$ and redetermining the efficiency times acceptance determination.
} 
This analysis also uses an isolation requirement on muons. The efficiencies for these isolations are calculated using the procedure outlined in the muon certification note [51]. The data used to derive the isolation efficiencies are MUOCERT output from MC p17.06.00 samples for the MC and MUOCERT output from p17.09.00 data for the data efficiency. Both are data sets of $Z / \gamma$ data produced from MUO_CERT. The efficiency distribution of the data is shown in Figure 7.6 and the efficiency distribution of the MC data is shown in Figure 7.7.

\section{Muon Isolation Efficiency}

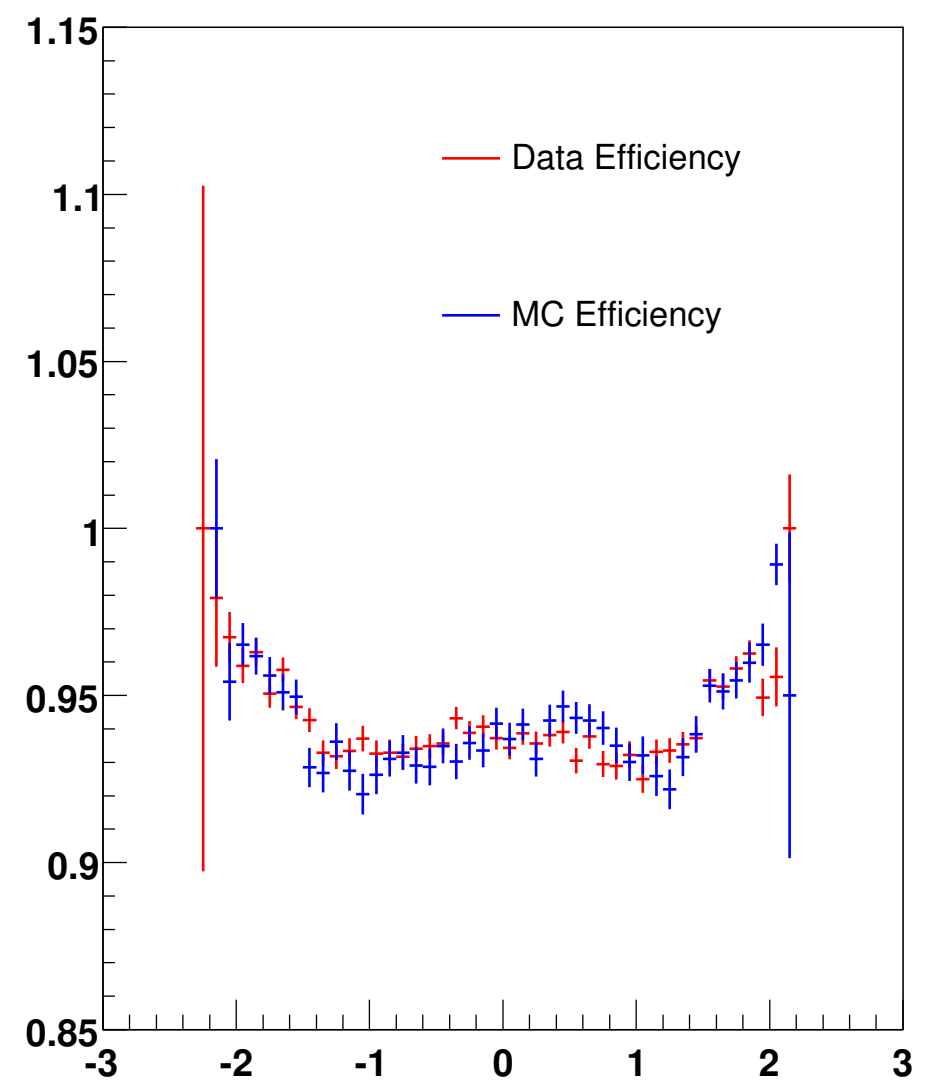

Figure 7.6: Shown are the isolation efficiencies for muons in data and MC. The muon isolation efficiencies are plotted bins of physics $\eta$.

These efficiencies are applied in the same manner as the other muon efficiencies.

The muon trigger efficiency is estimated by running $W Z$ PYTHIA MC and using PMCS 


\section{Muon Isolation MC Correction}

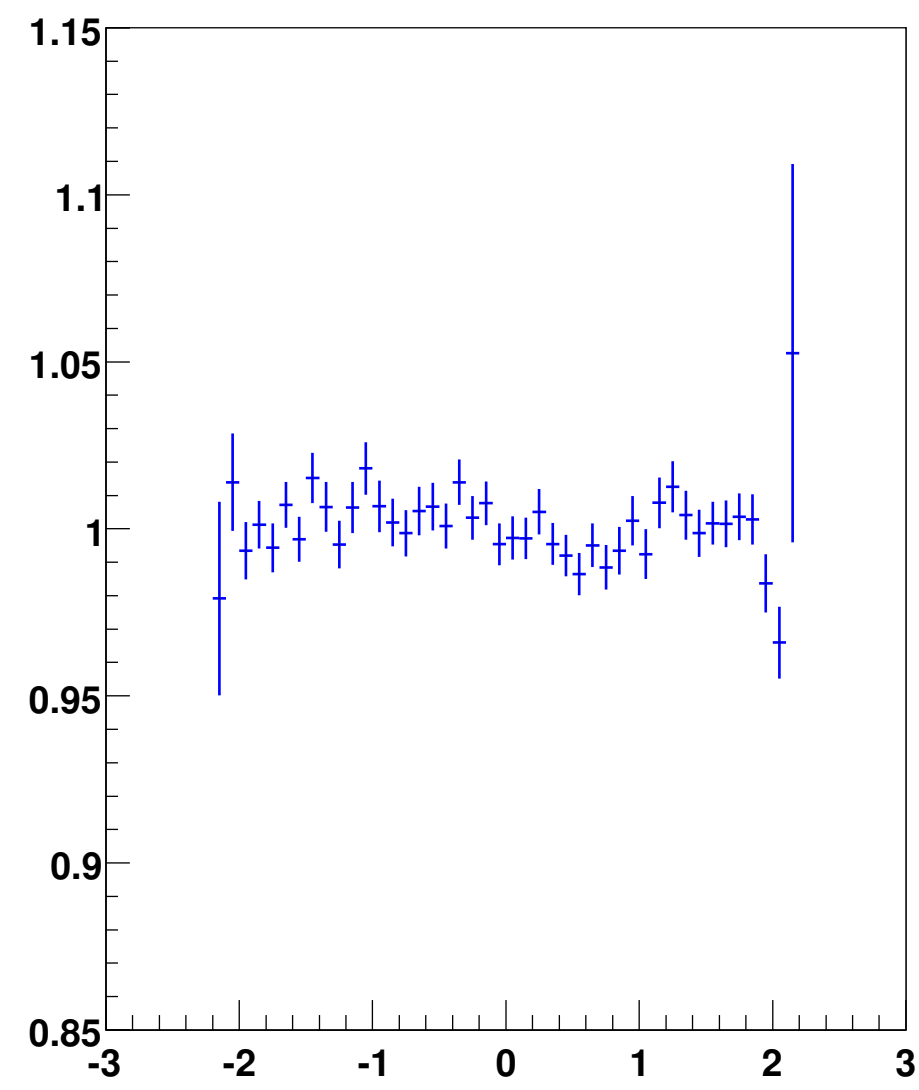

Figure 7.7: Shown are the isolation efficiency corrections for muons MC. The muon isolation efficiency corrections are plotted bins of physics $\eta$.

look up tables for the single muon trigger efficiencies. One single muon trigger is used per trigger version. The triggers used can be seen in Appendix D. The result of the study is that for the three muon signature the single muon trigger is $0.977 \pm 0.02$ efficient, while the two muon signature is $0.912 \pm 0.05$ efficient. These efficiencies are applied to the $\mu \mu \mu$ and the $\mu \mu e$ channels respectively.

\subsubsection{Total Acceptance times Efficiency}

After folding the total acceptance in with the lepton identification efficiencies and applying the trigger and charge cut efficiencies, the total acceptance times efficiency $\left(\mathcal{A}_{i} \times\right.$ 
$\left.\epsilon_{i}\right)$ per channel is given in Table 7.2.

Table 7.2: Presented are the total efficiencies, including the trigger and charge identification efficiencies, with uncertainties (stat. + syst.) along with the expected number of signal events and their uncertainties broken down into signal decay channel. The number of MC candidates used in determining the acceptance times efficiency is shown. The efficiency is presented when only the decay from $W$ and $Z$ bosons are directly to leptons $(e$ or $\mu$ ) are taken into account. Then the efficiency of the case where a boson decays to one or two $\tau$ 's which decay directly to leptons is also presented. Finally the total acceptance times efficiency including $\tau$ decays is presented.

\begin{tabular}{|c|c|c|c|c|}
\hline \hline Channel & $e e e$ & $e e \mu$ & $\mu \mu e$ & $\mu \mu \mu$ \\
Generated & 23116 & 23038 & 22954 & 23088 \\
Candidates & 3434.6 & 3615.6 & 3695.6 & 4409.8 \\
$\mathcal{A} \times \epsilon$ & 0.148 & 0.156 & 0.161 & 0.191 \\
Uncertainty & 0.013 & 0.10 & 0.017 & 0.029 \\
\hline Generated $\tau$ signal & 1396 & 1848 & 1864 & 1381 \\
Candidates from $\tau$ & 69.6 & 75.5 & 86.8 & 96.6 \\
$\mathcal{A} \times \epsilon$ from $\tau$ & 0.011 & 0.011 & 0.012 & 0.014 \\
Uncertainty & 0.006 & 0.002 & 0.003 & 0.005 \\
\hline Total $\mathcal{A} \times \epsilon$ & 0.159 & 0.167 & 0.173 & 0.205 \\
Uncertainty & 0.016 & 0.016 & 0.025 & 0.031 \\
\hline Normalized Expected Events & 2.26 & 2.23 & 2.18 & 2.54 \\
Uncertainty (Events) & 0.23 & 0.22 & 0.31 & 0.38 \\
\hline \hline
\end{tabular}

To calculate the total expected event yield we us the following equation,

$$
N_{S}=\mathcal{L} \times \sigma_{W Z} \times \operatorname{Br}(Z \rightarrow \ell \ell) \times B r(W \rightarrow \ell \nu) \times \mathcal{A} \times \epsilon
$$

Where $N_{S}$ is the expected number of signal events, $\sigma_{W Z}$ is the theoretical cross section for $W Z$ production, $\operatorname{Br}(Z \rightarrow \ell \ell)$ is the branching fraction for the $Z$ boson to decay to two muons or two electrons, $\operatorname{Br}(W \rightarrow \ell \nu)$ is the branching fraction for the $W$ boson to decay to a lepton neutrino pair, and $\mathcal{A} \times \epsilon$ is the total acceptance times efficiency. Table 7.3 shows the branching fractions for the four signal channels. The branching fraction for a $\tau$ to decay to an electron or muon has already been taken into account in the $\mathcal{A} \times \epsilon$ for the tau channels. Therefore the total $\mathcal{A} \times \epsilon$ is the sum of $\mathcal{A} \times \epsilon$ from $\tau$ and $\mathcal{A} \times \epsilon$ from the direct electron and muon channels.

As a cross check of the efficiencies for this analysis we measure the $Z$ boson yield in 
Table 7.3: Presented in this table are the branching fractions for the massive vector bosons to decay to electrons or muons and for the $\tau$ lepton to decay to an electron or muon. Also listed are the four branching fractions for the four channels. The branching fractions are taken from [1].

\begin{tabular}{|c|c|}
\hline Branching Fraction & Value \pm uncertainty \\
$B r(W \rightarrow e \nu)$ & $0.1075 \pm 0.0013$ \\
$\operatorname{Br}(W \rightarrow \mu \nu)$ & $0.1057 \pm 0.0015$ \\
$B r(Z \rightarrow e e)$ & $0.03363 \pm 0.00004$ \\
$B r(Z \rightarrow \mu \mu)$ & $0.03366 \pm 0.00007$ \\
$B r(\tau \rightarrow e)$ & $0.1784 \pm 0.0006$ \\
$B r(\tau \rightarrow \mu)$ & $0.1736 \pm 0.0006$ \\
\hline \hline Channel & Branching Fraction (uncertainty) \\
$e e e$ & $0.00361 \pm 0.00004$ \\
$e e \mu$ & $0.00355 \pm 0.00005$ \\
$\mu \mu e$ & $0.00362 \pm 0.00004$ \\
$\mu \mu \mu$ & $0.00355 \pm 0.00005$ \\
Total & $0.01435 \pm 0.00013$ \\
\hline
\end{tabular}

the two main samples used in the analysis, the 2EMhighpt sample and the 2MUhightpt sample. We use the following equations to determine the estimated yields:

$$
\begin{aligned}
\sigma_{Z \rightarrow e e} \mathcal{L} \epsilon_{T} \epsilon_{e}^{2} A_{Z \rightarrow e e} & =Y_{Z \rightarrow e e} \\
\sigma_{Z \rightarrow \mu^{+} \mu^{-}} \mathcal{L} \epsilon_{T} \epsilon_{\mu}^{2} \epsilon_{q} A_{Z \rightarrow \mu^{+} \mu^{-}} & =Y_{Z \rightarrow \mu \mu}
\end{aligned}
$$

where $\sigma_{Z \rightarrow e e}$ and $\sigma_{Z \rightarrow \mu^{+} \mu^{-}}$are the cross section for $Z \rightarrow e e$ and $Z \rightarrow \mu \mu$ production, $\mathcal{L}$ is the integrated luminosity for the data sets, $\epsilon_{T}$ is the trigger efficiency, $\epsilon_{e}$ is the electron ID efficiency, $\epsilon_{\mu}$ is the $\mu$ ID efficiency and $A_{Z \rightarrow e e}$ and $A_{Z \rightarrow \mu^{+} \mu^{-}}$are the measured acceptance of the $Z$ selection. The acceptances are measured from $Z \rightarrow \ell \ell$ Monte Carlo samples with the request ID's of $33670,30604,38770,38771,38772$ and 38773 . The values for all of these parameters are given in Table 7.4.

The amount of signal and background are determined from the integrals of the fits. After subtracting off the background from the $Z \rightarrow$ ee peak we are left with 74964 events with in our mass window. The backgrounds are determined by the exponential fit of the background underneath the $Z$ peaks shown in Figures 7.1 and 7.2. The background within 
Table 7.4: Summarized in this table are the values of the parameters used in determining the number of expected $Z$ bosons in the dilepton decay channels.

\begin{tabular}{|c|c|}
\hline Parameter & Value (uncertainty) \\
\hline$e e$ Trigger $\left(\epsilon_{T}\right)$ & $0.99 \pm 0.01$ \\
$\mu \mu e$ Trigger $\left(\epsilon_{T}\right)$ & $0.91 \pm 0.05$ \\
Charge Cut Efficiency $\left(\epsilon_{q}\right)$ & $0.99 \pm 0.01$ \\
electron efficiency $\left(\epsilon_{e}\right)$ & $0.91 \pm 0.02$ \\
muon efficiency $\left(\epsilon_{\mu}\right)$ & $0.90 \pm 0.02$ \\
$A_{Z \rightarrow \mu^{+} \mu^{-}}$ & $0.374 \pm 0.001$ \\
$A_{Z \rightarrow e e}$ & $0.329 \pm 0.001$ \\
$\sigma_{Z \rightarrow e e}$ & $250 \pm 18 \mathrm{pb}$ \\
$\sigma_{Z \rightarrow \mu^{+} \mu^{-}}$ & $250 \pm 18 \mathrm{pb}$ \\
\hline
\end{tabular}

the mass window is $2781 \pm 80$ events. The uncertainty is taken from the fit. The expected yield when using Equation 7.6 above is $69857 \pm 6747$ events. For the $\mu$ channel; after subtracting off the background from the $Z \rightarrow \mu^{+} \mu^{-}$peak we are left with 74081 events. The background with in the mass window is determined to be $2690 \pm 1241$ events. The uncertainty is taken from the fit. The expected yield determined from Equation 7.7 above is $76331 \pm 10251$ events. The event yields are summarized in Table 7.5. The contributions to the errors of these expected yields are shown in Table 7.6.

Table 7.5: In this table the $Z \rightarrow \ell \ell$ event yields in data are compared with expectations determined using Equations 7.6 and 7.7. The systematic uncertainties on the data are taken from the uncertainties on the background subtraction.

\begin{tabular}{|c|c|c|}
\hline Channel & Expectation & Data \pm Stat. \pm Syst. \\
\hline$Z \rightarrow e e$ & $72048 \pm 7389$ & $74964 \pm 274 \pm 80$ \\
$Z \rightarrow \mu^{+} \mu^{-}$ & $64962 \pm 7858$ & $74081 \pm 272 \pm 1241$ \\
\hline
\end{tabular}

\subsection{Background}

The observation of $W Z$ production using trilepton final states greatly depends on keeping backgrounds at a small level. Using the trilepton final states eliminates practically all but a few sources of physics background: $t \bar{t}, Z Z, Z \gamma, Z+$ jets and Drell-Yan. The first can be separated from the signal by taking advantage of the large hadronic energy 
Table 7.6: Presented in this table is the breakdown of the uncertainty in the expected $Z$ boson yield in the dilepton decay channels. The uncertainties are shown in percentages and in events.

\begin{tabular}{|c|c|c|}
\hline Source & Value (\%) & Value (event) \\
\hline ee Trigger $\left(\epsilon_{T}\right)$ & $1 \%$ & 699 \\
$\mu \mu e$ Trigger $\left(\epsilon_{T}\right)$ & $5 \%$ & 3817 \\
Charge Cut Efficiency $\left(\epsilon_{q}\right)$ & $3 \%$ & 2290 \\
electron efficiency $\left(\epsilon_{e}\right)$ & $4 \%$ & 2882 \\
muon efficiency $\left(\epsilon_{\mu}\right)$ & $4 \%$ & 2598 \\
$A_{Z \rightarrow \mu^{+} \mu^{-}}$ & $0.3 \%$ & 229 \\
$A_{Z \rightarrow e e}$ & $0.3 \%$ & 210 \\
$\sigma_{Z \rightarrow e e}$ & $7 \%$ & 4890 \\
$\sigma_{Z \rightarrow \mu^{+} \mu^{-}}$ & $7 \%$ & 5343 \\
$\mathcal{L}_{e e}$ & $6.5 \%$ & 4541 \\
$\mathcal{L}_{\mu \mu}$ & $6.5 \%$ & 4962 \\
\hline
\end{tabular}

produced in its decay. The $t \bar{t}, Z Z, Z \gamma$ and Drell-Yan backgrounds are estimated using MC. The $Z+$ jets can be estimated using data. This section will describe how these backgrounds are estimated.

\subsubsection{QCD Backgrounds}

For this analysis, a fake electron and muon are defined as any electron or muon that is not a decay product of $W$ or $Z$ bosons. One way to measure the rate at which a fake electron or muon are produced is to count the times that such objects occur in association with another real object that is not part of signal signature. One possibility could be to determine the rate that a fake lepton occurs in association with a jet. Jets are both abundant in our signal sample and are known to mimic leptons at a small rate.

Dijet events from the QCD skim are used to estimate these fake probabilities. Described first is the manner in which the probability for an electron to mimic a jet is determined. We then discuss the method used to determine the probability that jet will fake a muon. The two methods differ in that an EM object is always a jet object, where as a muon is not always a jet object.

When looking at the dijet events the first jet (tag jet) has to pass the jet quality 
cuts, defined by the Jet ID group [62]. The requirement for the second jet (probe jet) is that it be in the opposite side of the detector in $\phi$ (where opposite side is defined as ||$\phi_{\text {tag }}-\phi_{\text {probe }}|-\pi|<0.4$ radians) and the $p_{T}$ ratio of the tag jet to the probe jet be smaller than 3.0. Finally the $\mathbb{E}_{T}$ must be less than $10 \mathrm{GeV}$ so that contamination from $W \rightarrow \ell \nu$ events is minimal. There is no other requirement. The second jet sample is thus an unbiased jet sample. The tag jet requirements are summarized here:

- A tag jet must pass all of the Jet ID good jet requirements.

- A tag jet must be the highest $p_{T}$ jet in the event ${ }^{5}$.

- A tag jet must have $p_{T}>15.0 \mathrm{GeV}$.

- A tag jet must be in the central detector: $|\eta|<1.1$.

- A good dijet event must have $\mathbb{E}_{T}<10 \mathrm{GeV}$.

From this set of unbiased jet data, we will calculate the probability for a jet to fake a lepton. To determine the total number of QCD events in our signal sample we apply these fake probabilities to two normalization samples. The first is the $Z \rightarrow \ell \ell+$ jets sample, and the second is the $e+\mu+$ jets sample. This normalization sample spans the same luminosity that the signal samples span. The jets here are required to be good jets and have a $p_{T}$ larger than $15.0 \mathrm{GeV}$. The first normalization sample is taken from the two lepton high $p_{T}$ skims and are analyzed in an identical manner as the signal is, except that the jets are treated as a third lepton. The second normalization sample used the $e+\mu$ skimmed data and is also analyzed in an identical manner as the signal except that the jets are treated one leg of the $Z \rightarrow \ell \ell$ decay. This sample accounts for the situation where the $W$ boson from our candidate events are real, but the $Z$ boson is fake.

\footnotetext{
${ }^{5}$ The highest $p_{T}$ jet in each QCD event is assumed to be the trigger object for the event.
} 


\section{Fake electron probability}

Once a probe jet is found, a search cone of $\Delta \mathcal{R}=0.7$ (centered around the probe jet) is used to look for a good electron. To determine the systematic effects of this background estimation method, the search cone is varied from a cone of size of 0.5 to 0.9 . The largest difference in fake probability is used as the systematic error. The fake probability is determined as a function of detector $\eta$ and calculated using the equation

$$
P_{i}(\text { fake electron }: \text { jet })=\frac{\sum_{\text {fake electrons }} e\left(\eta_{i}\right)}{\sum_{\text {probe jets }} j e t\left(\eta_{i}\right)},
$$

where $i$ corresponds to the $\eta$ bin.

The average probability is, conservatively, on the order of $8 \times 10^{-4}$. The probability for a jet to fake an electron is shown in Figure 7.8. A sample of $Z \rightarrow \ell \ell+$ jets is selected where the $Z$ boson selection requirements are identical to our signal and the jet selection requirements are identical to ones just described. The probability for a jet to fake an electron is multiplied by the distribution of jets in the $Z \rightarrow \ell \ell+$ jets sample, as described by the equation

$$
N_{\text {background }}\left(\eta_{i}\right)=P_{i}(\text { fake electron : jet }) \times \operatorname{jet}\left(\eta_{i}\right)
$$

where $P_{i}$ (fake electron : jet) is determined from Equation 7.8 and jet $\left(\eta_{i}\right)$ is the jet distribution in the $Z \rightarrow \ell \ell+$ jets samples. The binned background contribution of $e e+$ jets to the eee signal events is shown in Figure 7.9. The binned background contribution of $\mu \mu+$ jets to the $\mu \mu e$ signal events is shown in Figure 7.10. The binned background contribution of $e+\mu+$ jets to the $e e \mu$ signal events is shown in Figure 7.11. 


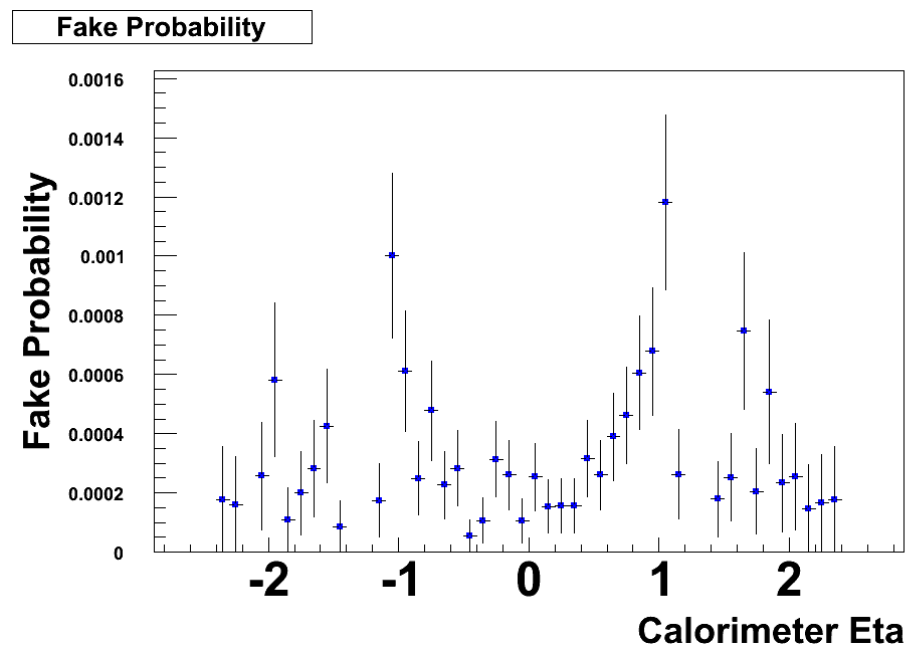

Figure 7.8: The electron fake probability as a function of detector $\eta$. The error bars are statistical error bars.

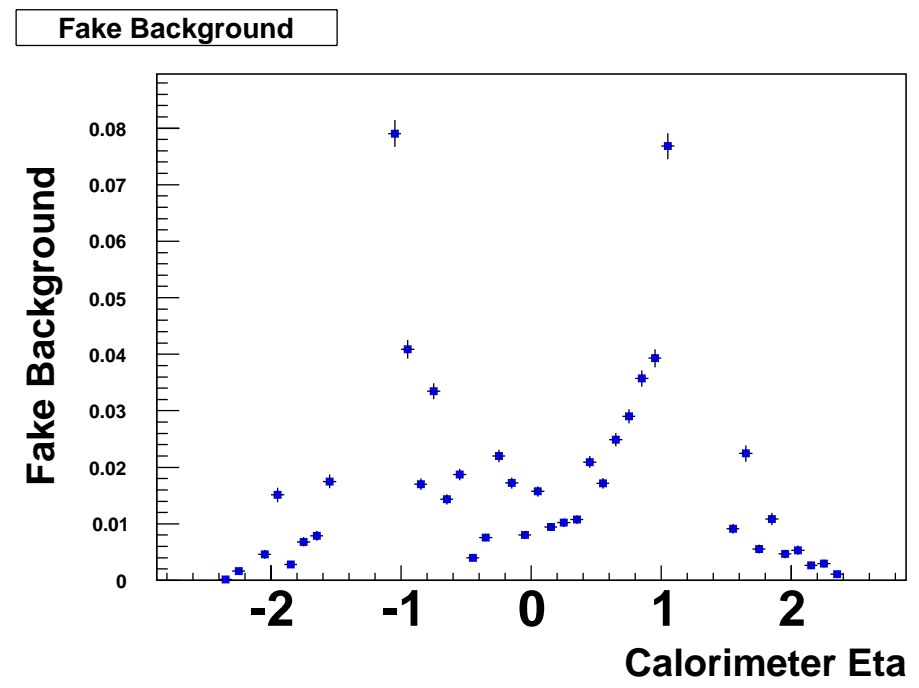

Figure 7.9: The eee QCD fake background vs. detector $\eta$ determined from the ee+ jets sample. The error bars are statistical error bars.

Fake muon probability

When looking for fake muons, the tag jet requirements are the same as before, but the probe jet requirements are different. When a tag jet is present then a muon or probe jet is searched for in a $\phi$ wedge in the opposite side of the detector. Here $\phi$ wedge is defined as ||$\phi_{\text {tag }}-\phi_{\text {probe }}|-\pi|<0.9$ radians. If a probe jet or a muon is found then they are both counted. The $\phi$ wedge is varied from 0.9 radians to \pm 0.2 radians to estimate the 


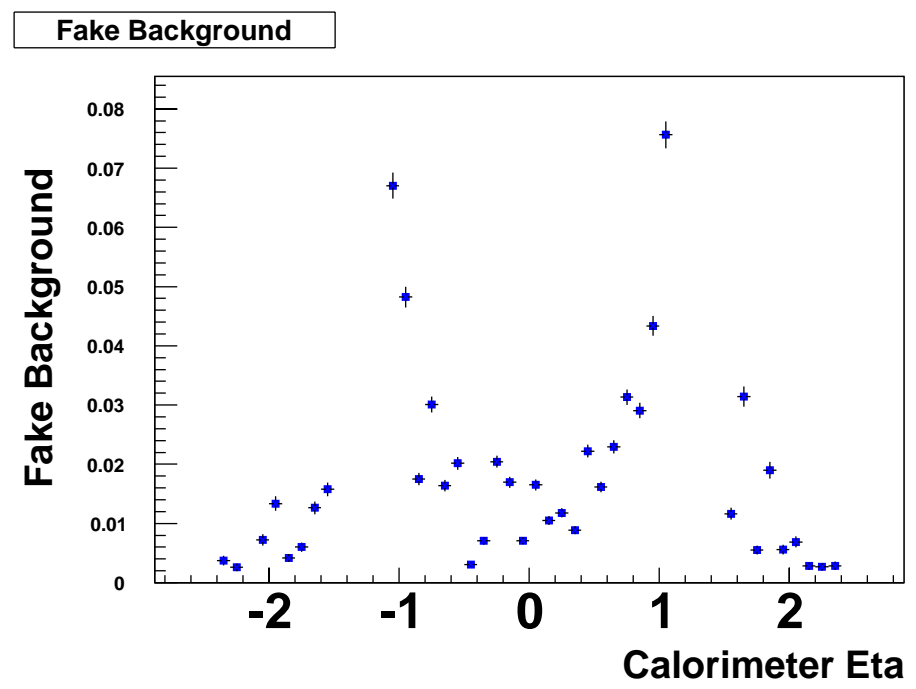

Figure 7.10: The $\mu \mu e$ QCD fake background vs.detector $\eta$ determined from the $\mu \mu+$ jets sample. The error bars are statistical error bars.

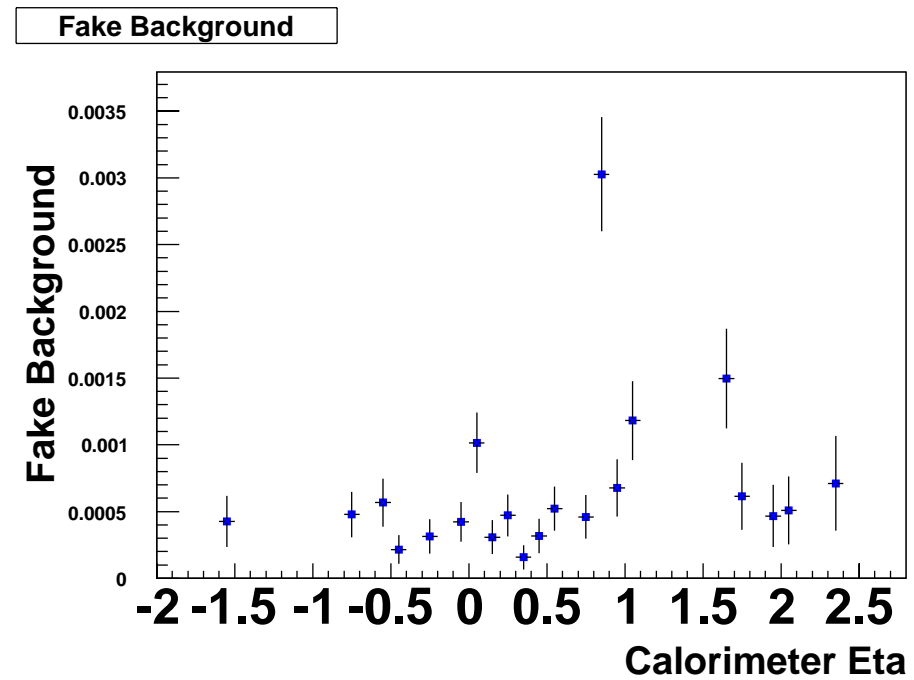

Figure 7.11: The $e e \mu$ QCD fake background vs.detector $\eta$ determined from the $e+\mu+$ jets sample. The error bars are statistical error bars.

systematics of this method. The muon fake rate is then calculated by

$$
P_{i}(\text { fake muon }: \text { jet })=\frac{\sum_{\text {fake muons }} \mu}{\sum_{\text {probe jets }}+\sum \text { fake muons }},
$$

The fake muon probability does not have enough statistics to justify a binned or parameterized probability. Based on the number of muons found in the fake sample, 
the fake muon probability is $3.9 \times 10^{-5} \pm 0.9 \times 10^{-5}$. The backgrounds from fake muons are shown in Table 7.12 and are calculated using the equation,

$$
N_{\text {background }}=P(\text { fake muon }: \text { jet }) \times N_{\text {jet }}
$$

where $N_{\text {jet }}$ is the number of jets found within the muon acceptance of the $Z \rightarrow \ell \ell+$ jets data sample described in the electron fake rate discussion.

\subsubsection{QCD Background Cross Check}

As the jet contribution to the background is the largest source of background a method was developed to cross check the QCD background estimate. This method uses bad leptons as opposed to jets to determine the background. The jet tagged QCD skim sample is still the sample used to determine the fake ratio. A major difference among the methods is that the energy scale for the cross check is more like the signal where as this is not true for the jet method. Here the fake ratio is number of good leptons to the number of bad leptons, defined below. The normalization sample is $Z+$ bad leptons that satisfy all of the analysis cuts except that the third lepton is required to be a bad lepton.

The bad electron identification requirements are defined below.

- Isolation ratio $<0.2$.

- EM Fraction $>0.9$.

- $p_{T}>15 \mathrm{GeV}$

- Likelihood $>0$

- Likelihood $<0.1$

A good electron is the same electron as defined in the analysis. The major difference between a bad electron and good electron are the likelihood requirements.

The bad muon identification requirements are defined below. 
- Loose quality

- Cosmic Veto applied

- Nseg $>0$

- etHalo $>4.0$

- etTrkCone $5>5.0$

- $p_{T}>15 \mathrm{GeV}$

A good muon requires the same muon identification requirements as in the analysis. The major difference between a good muon and a bad muon are the isolation requirements.

To get the ratio of bad leptons to good leptons the QCD skim was utilized. First the highest $p_{T}$ jet, which is a jet that also satisfies the good jet requirements defined by the Jet ID group, is found. This jet is assumed to be the trigger object of the event. Next the event is required to have $\mathbb{E}_{T}$ less than $20 \mathrm{GeV}$. Next bad leptons that are in the opposite side of the detector in $\phi,|| \phi_{j e t}-\phi_{\text {lepton }}|-\pi|>\frac{\pi}{2}$ are searched for and plotted as function of lepton detector $\eta$ and lepton $p_{T}$. The distributions are shown in Figures 7.12 to 7.19.

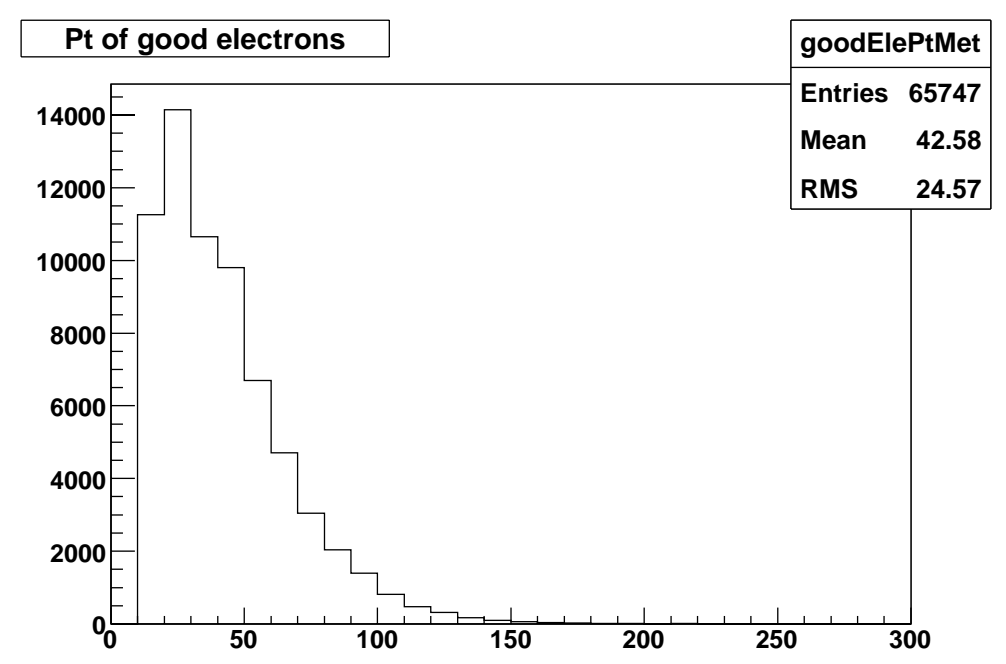

Figure 7.12: Distribution of good electron $p_{T}$ in the QCD sample. 


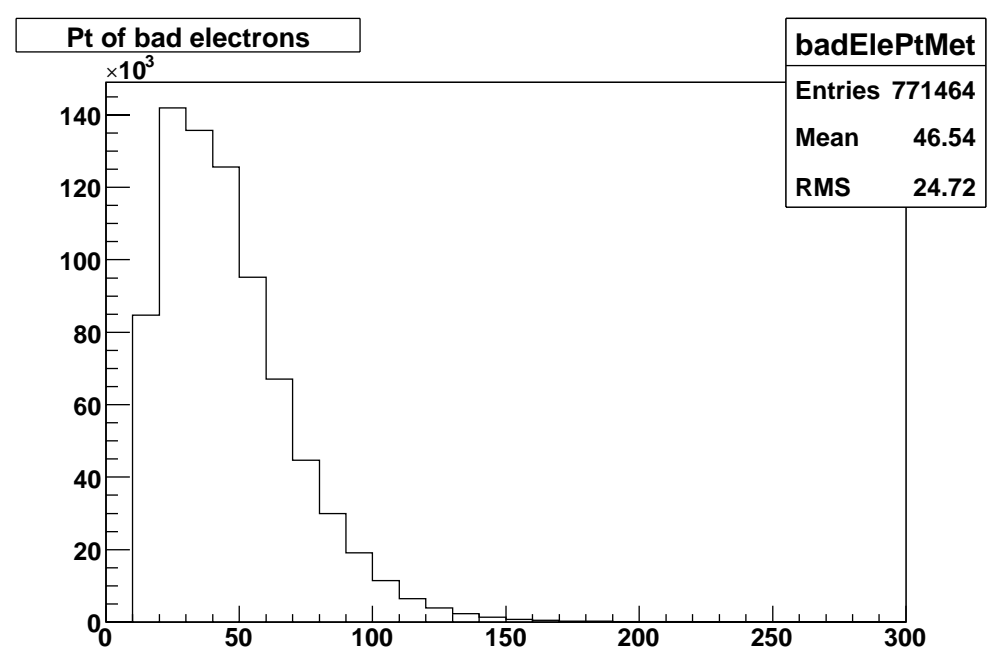

Figure 7.13: Distribution of bad electron $p_{T}$ in the QCD sample.

The fake ratios are determined by dividing the bad lepton distributions by the good lepton distributions. The fake ratios are shown in Figures 7.20 to 7.23.

$Z+$ bad lepton events were then searched for in the 2EMhighpt and 2MUhighpt skims. The normalization distributions are shown in Figures 7.24 - 7.29. 


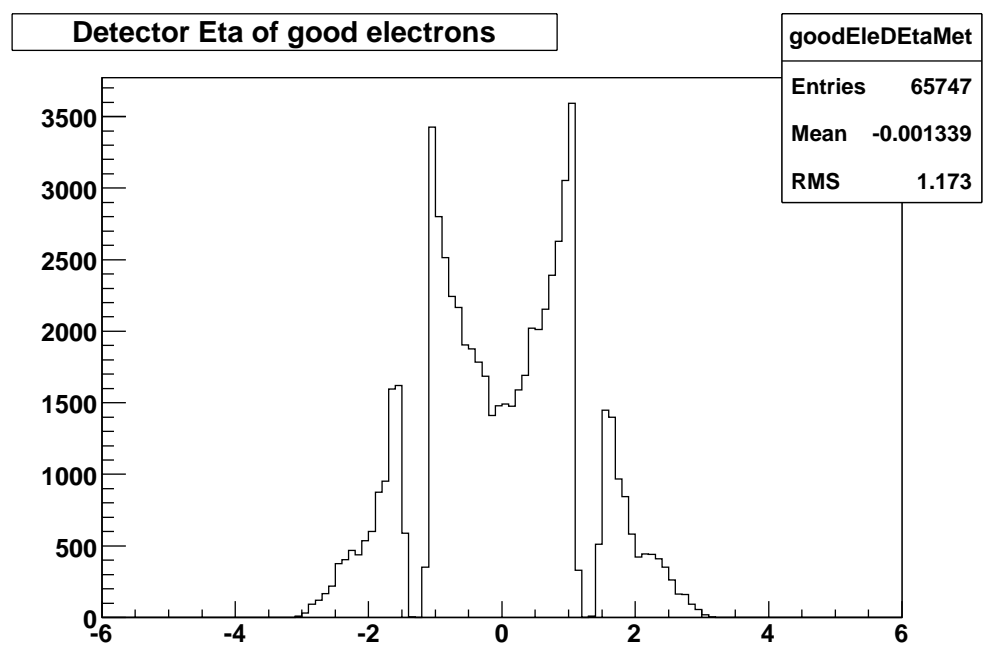

Figure 7.14: Distribution of good electrons in detector $\eta$ in the QCD sample.

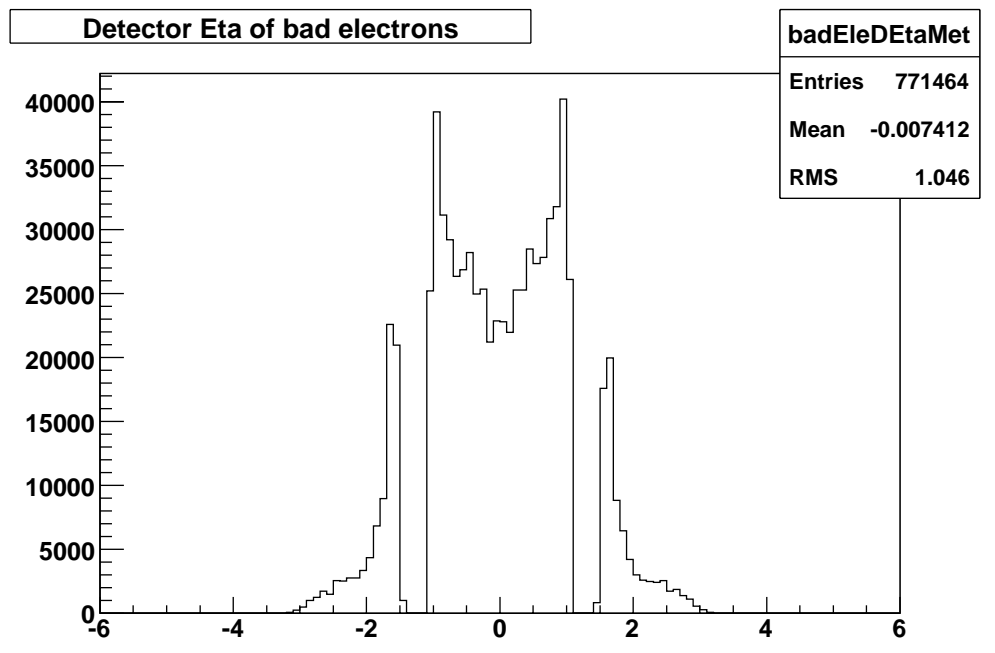

Figure 7.15: Distribution of bad electron detector $\eta$ in the QCD sample. 


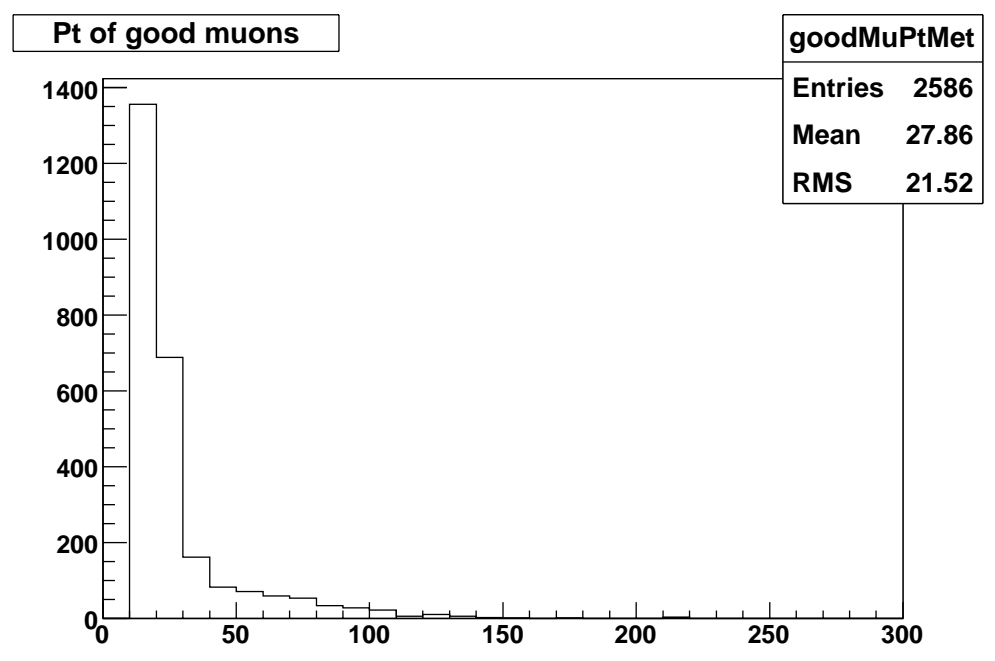

Figure 7.16: Distribution of good muon $p_{T}$ in the QCD sample.

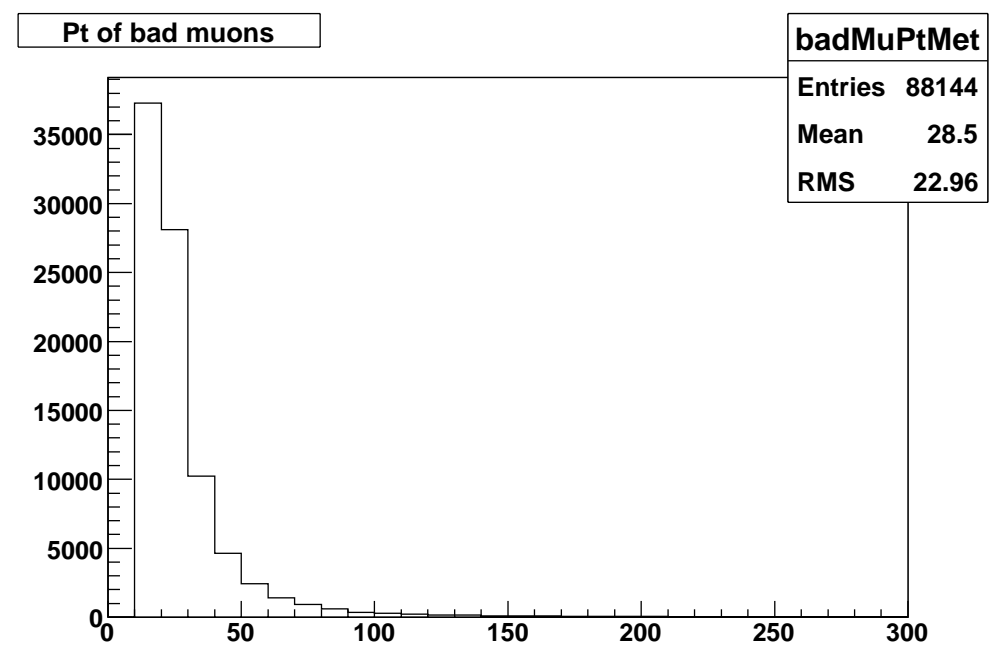

Figure 7.17: Distribution of the bad muon $p_{T}$ in the QCD sample. 


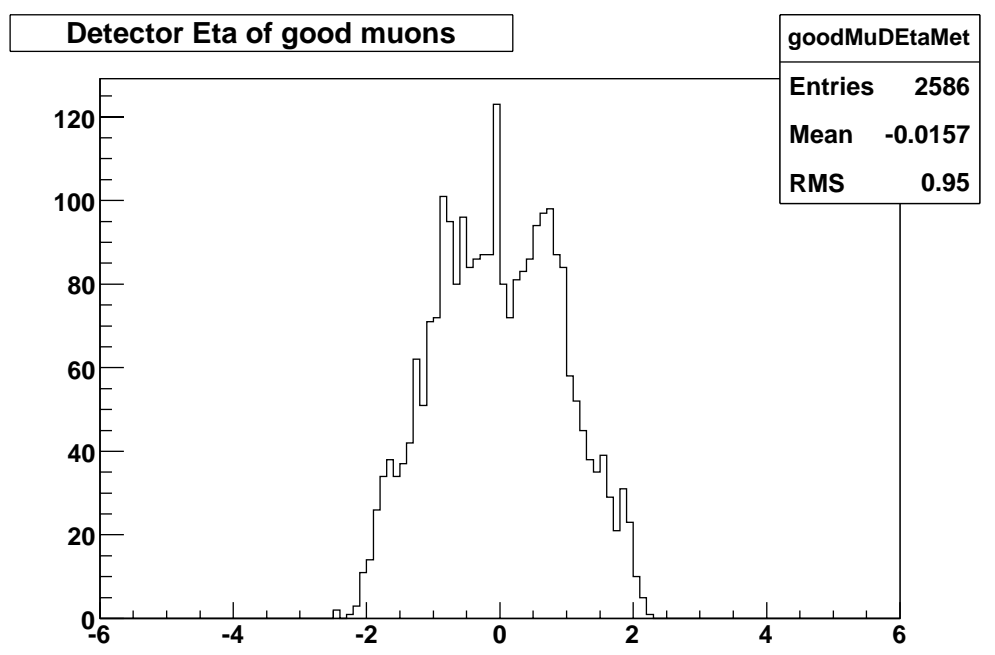

Figure 7.18: Distribution of the good muon detector $\eta$ in the QCD sample.

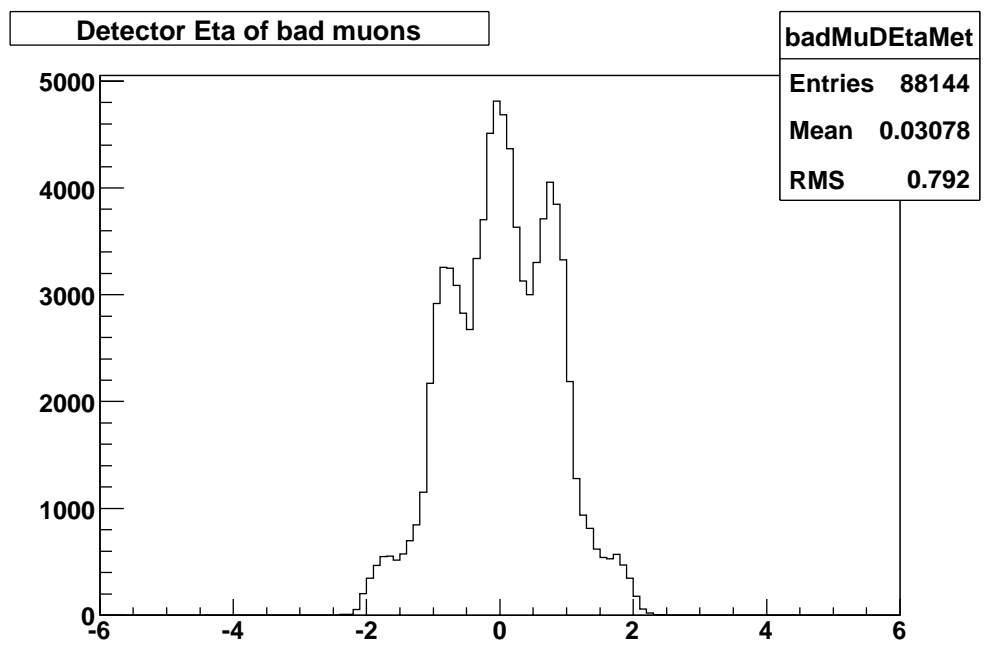

Figure 7.19: Distribution of the bad muon detector $\eta$ in the QCD sample. 


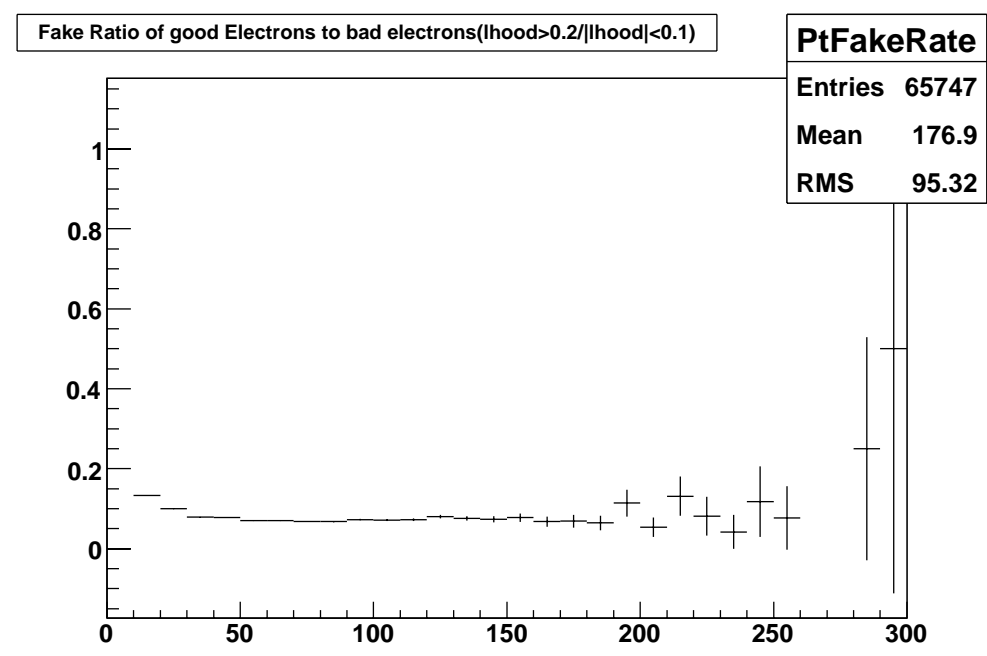

Figure 7.20: Distribution of electron fake rate in $p_{T}$. The error bars are the statistical Gaussian uncertainties.

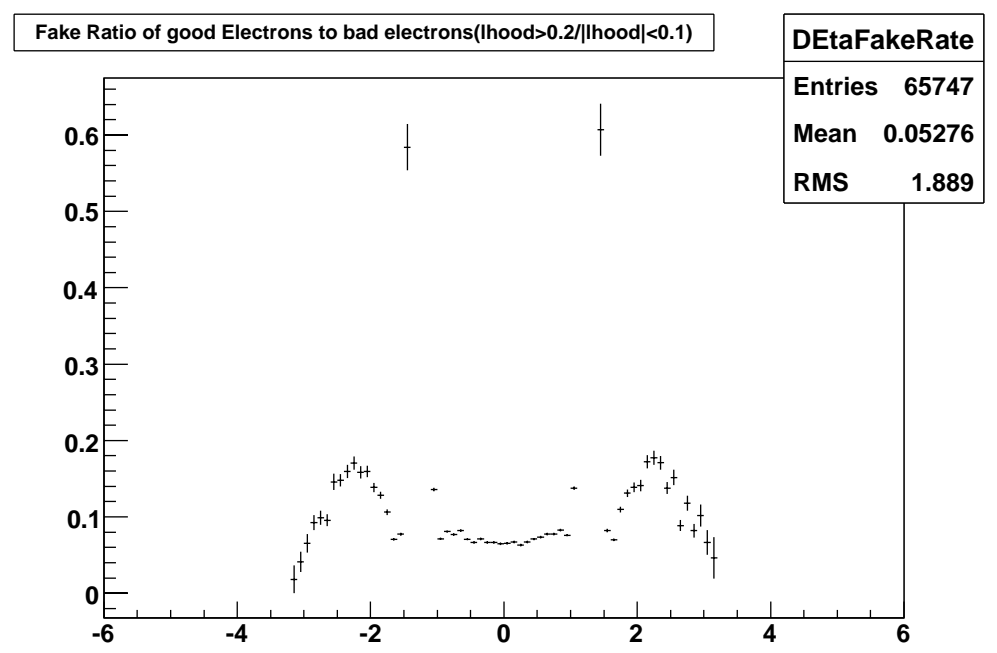

Figure 7.21: Distribution of electron fake rate in detector $\eta$. The error bars are the statistical Gaussian uncertainties. 


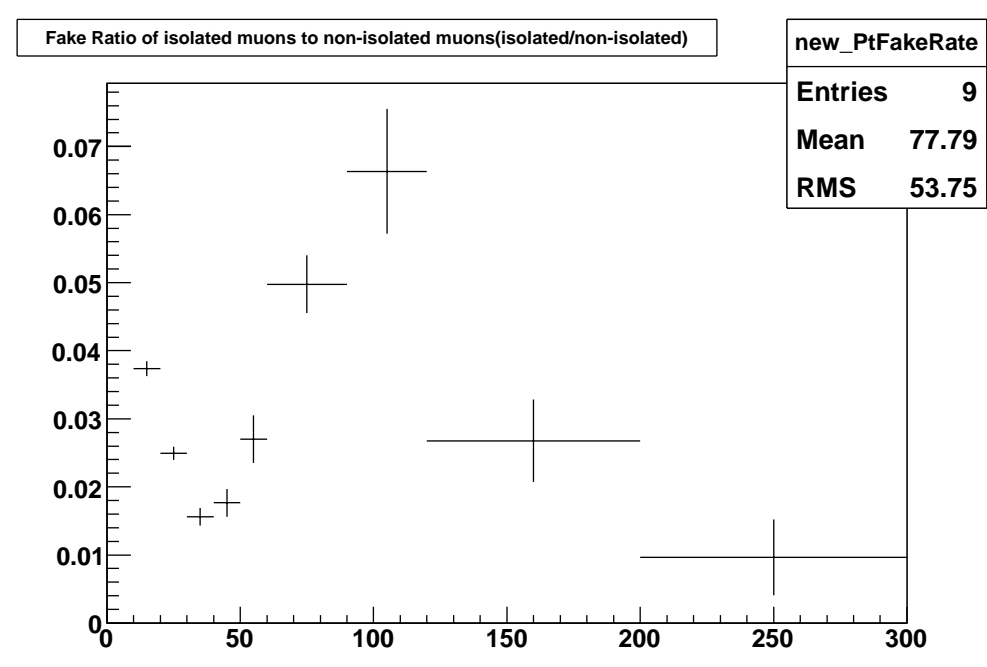

Figure 7.22: Distribution of muon fake rate in $p_{T}$. The error bars are the statistical Gaussian uncertainties.

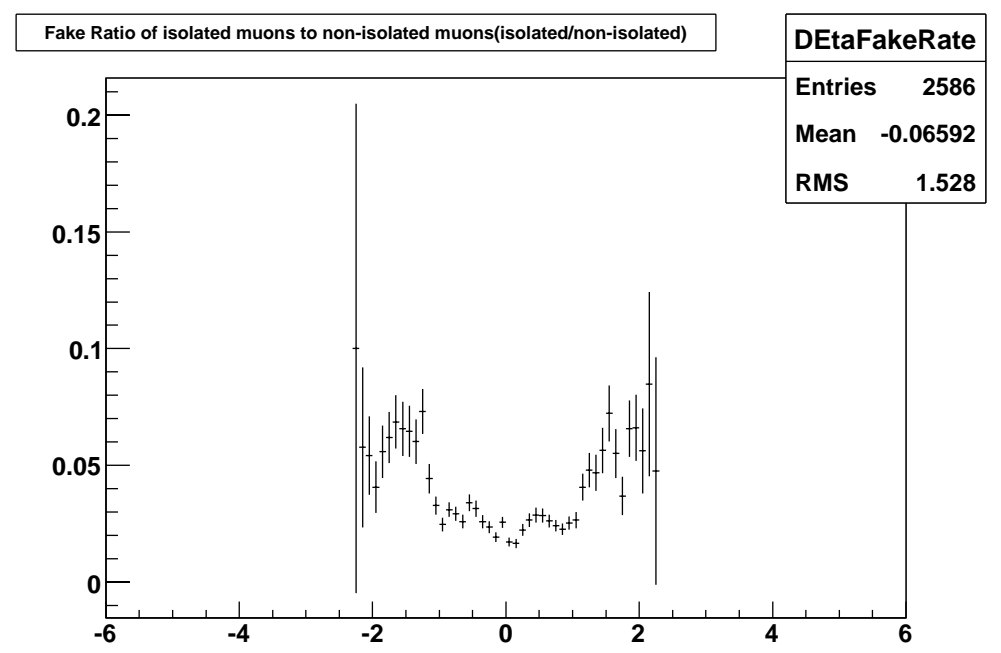

Figure 7.23: Distribution of muon fake rate in detector $\eta$. The error bars are the statistical Gaussian uncertainties. 


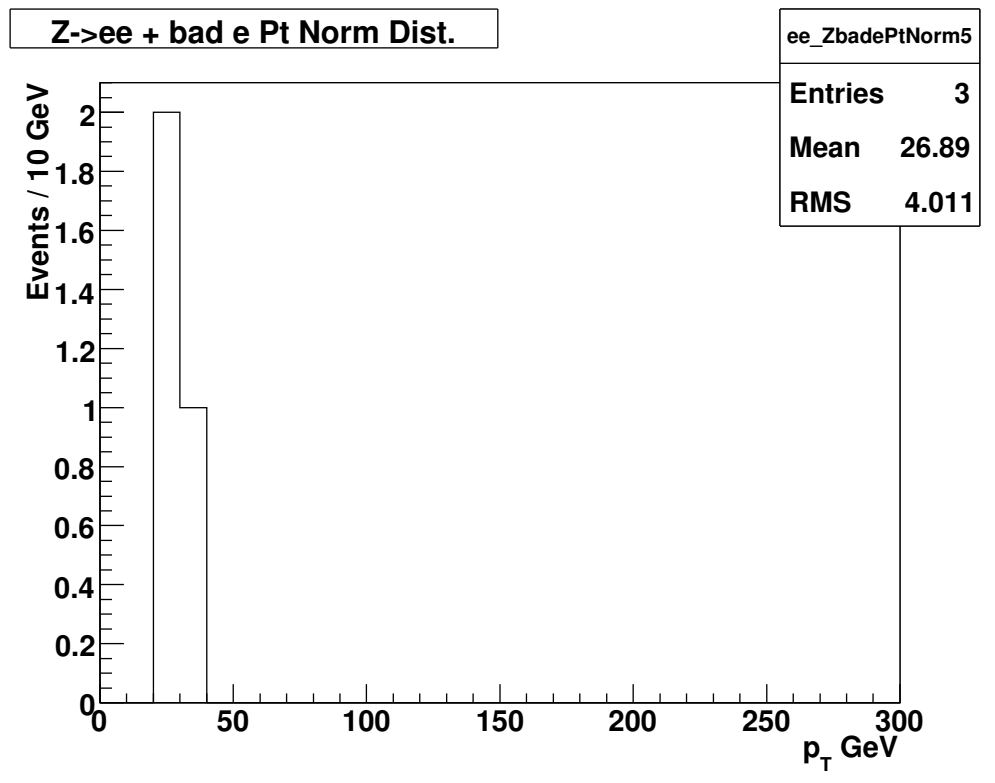

Figure 7.24: Normalization sample of $e e+$ bad $e$ events distributed in $p_{T}$.

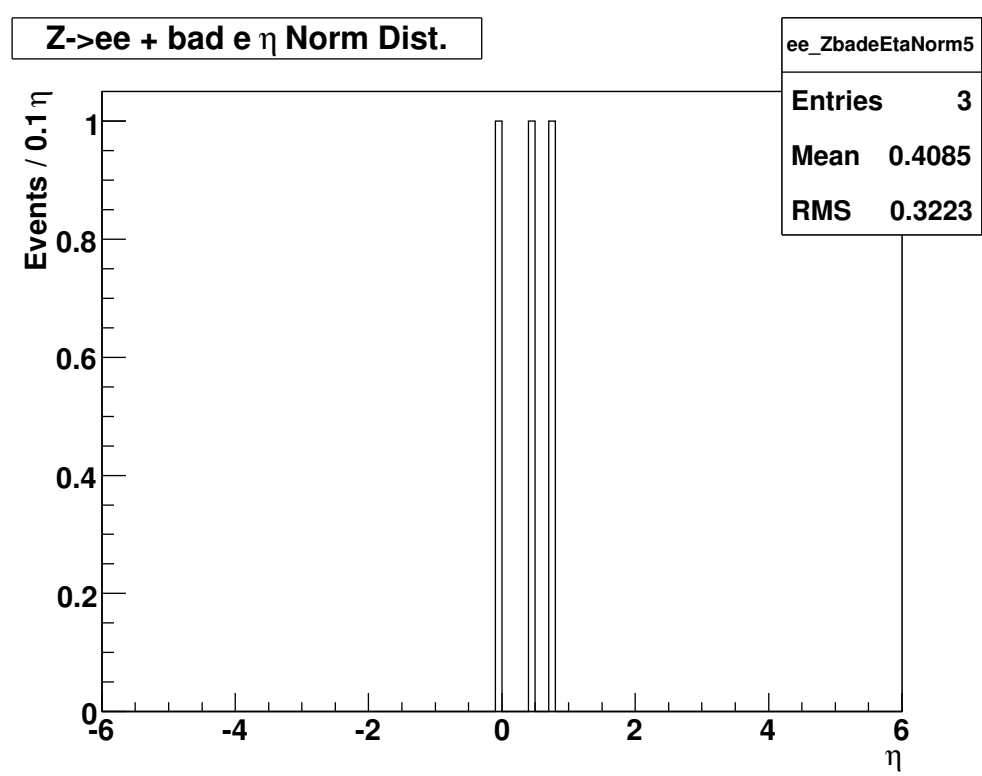

Figure 7.25: Normalization sample of $e e+$ bad $e$ events distributed in detector $\eta$. 


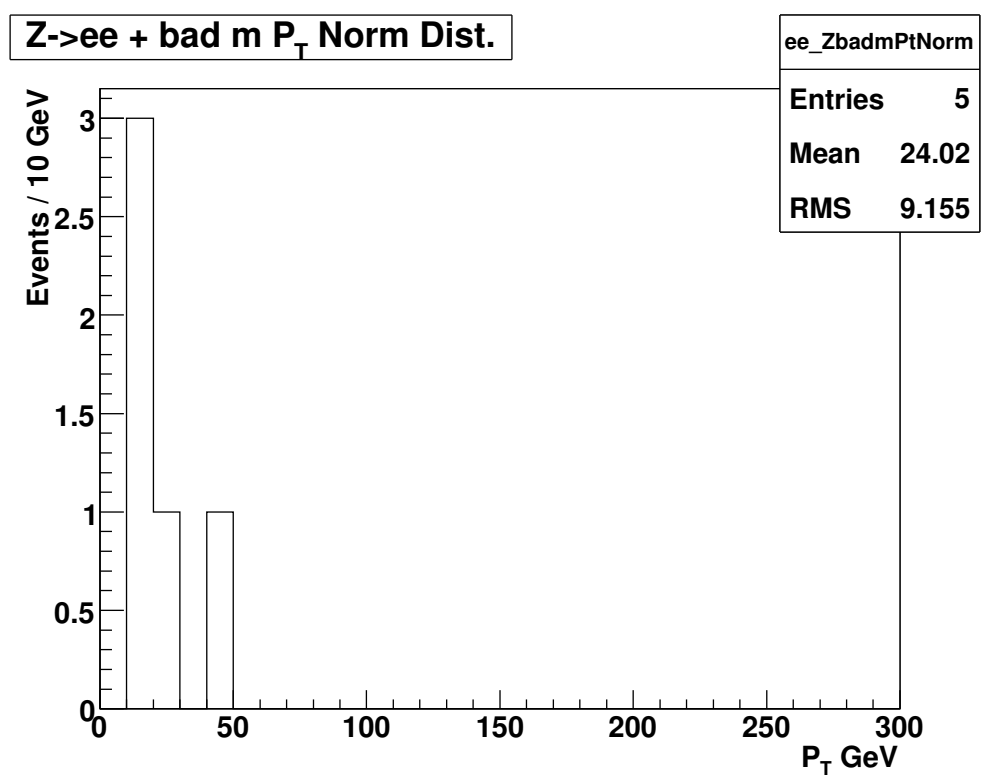

Figure 7.26: Normalization sample of $e e+$ bad $\mu$ events distributed in $p_{T}$.

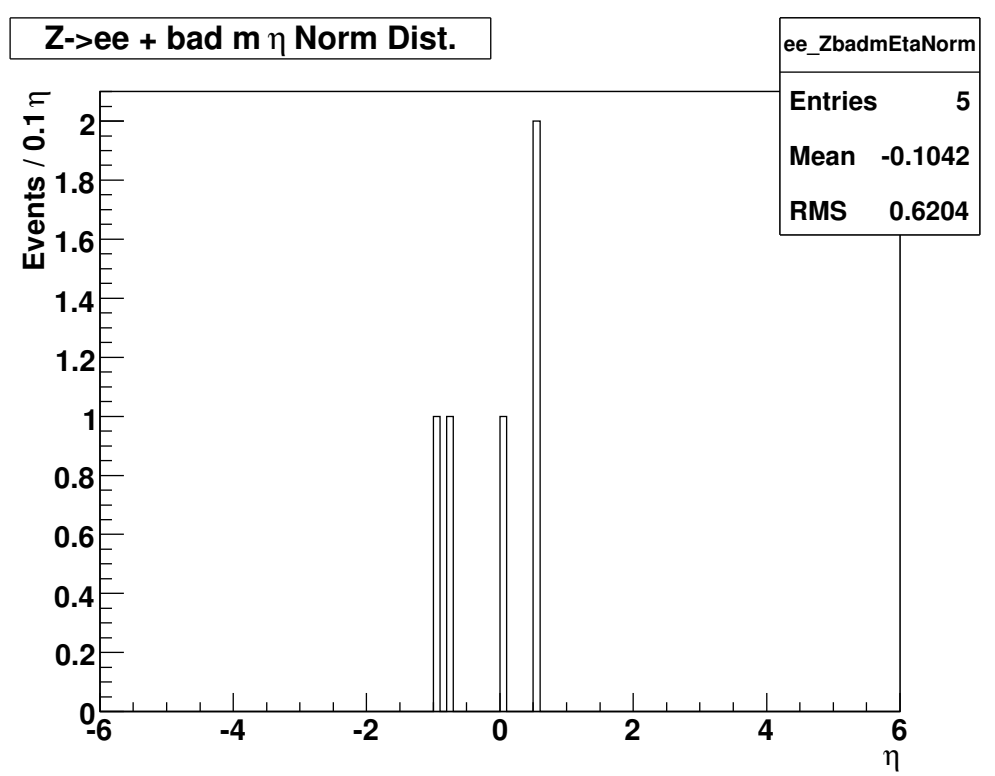

Figure 7.27: Normalization sample of $e e+$ bad $\mu$ events distributed in detector $\eta$. 


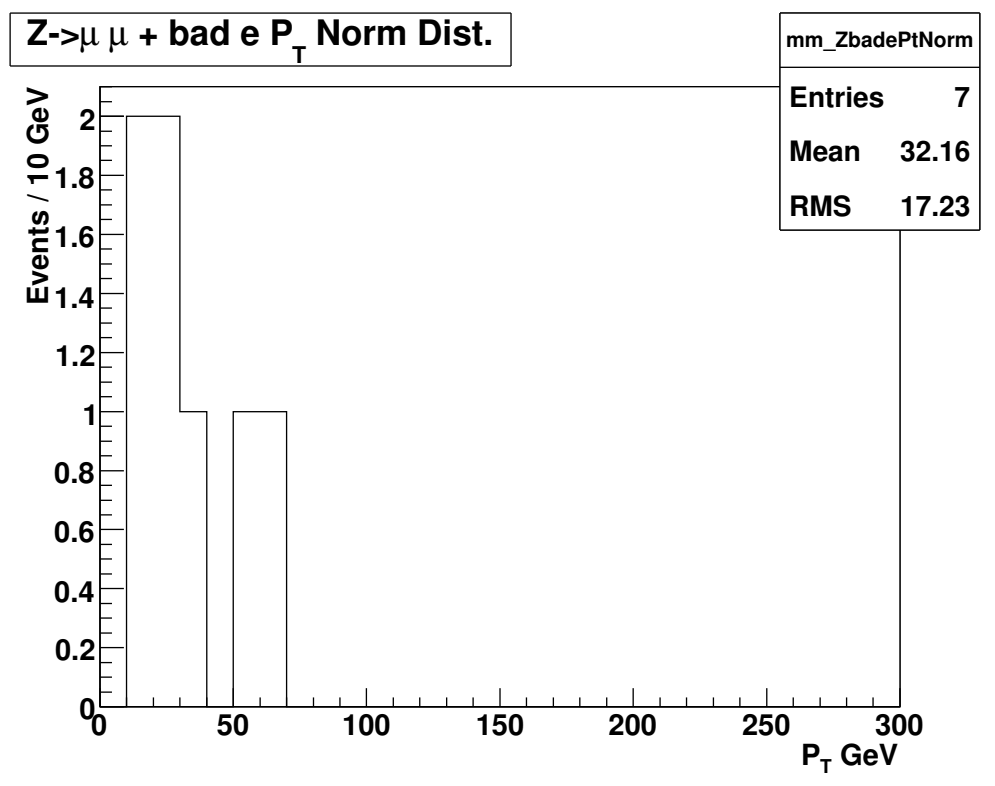

Figure 7.28: Normalization sample of $\mu \mu+$ bad $e$ events distributed in $p_{T}$.

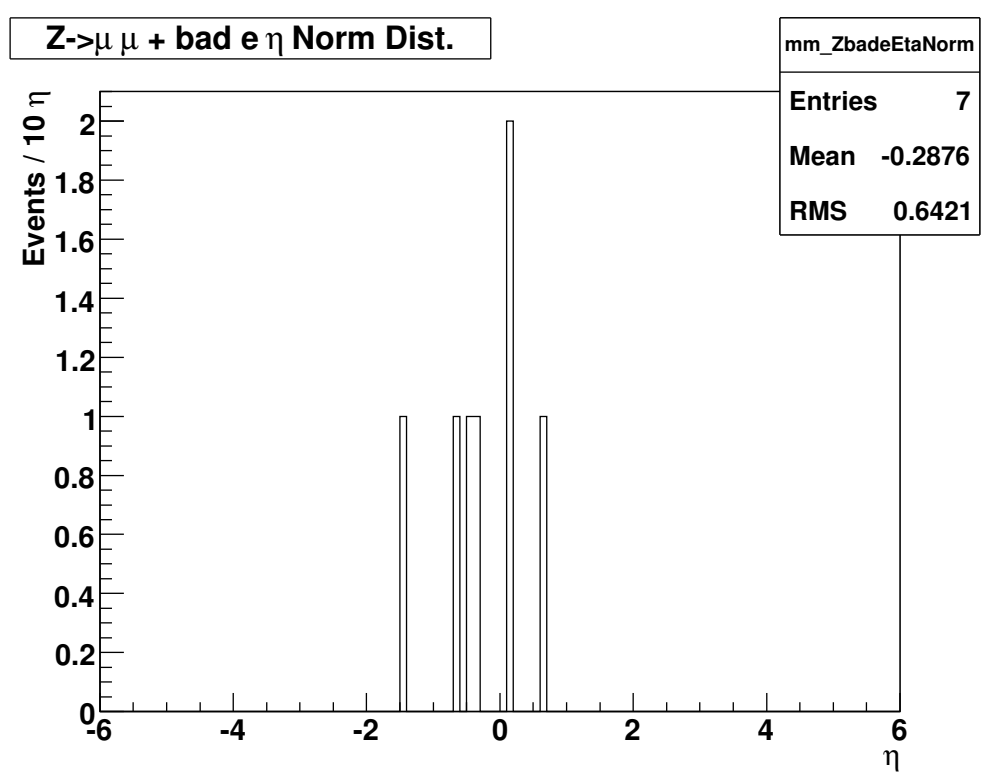

Figure 7.29: Normalization sample of $\mu \mu+$ bad $e$ events distributed in detector $\eta$. 


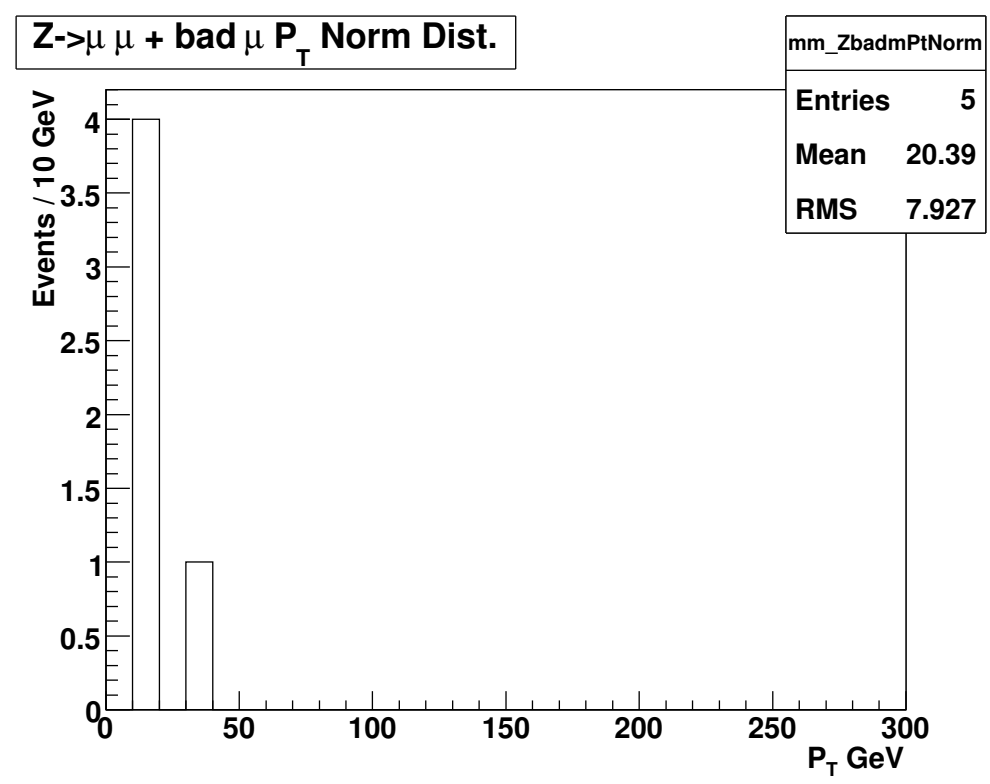

Figure 7.30: Normalization sample of $\mu \mu+$ bad $\mu$ events distributed in $p_{T}$.

These normalization samples have a small number of events which limits statistical power of this cross check. The resulting background estimates are shown in Figure 7.32 through Figure 7.39.

Taking the background estimate differences between the $p_{T}$ distributions and detector $\eta$ distributions as the systematic bounds on the estimates, Table 7.7 compares the background estimates between the method using jets and the method using bad leptons.

Note that the information in Table 7.7 does not include information from the $e+\mu+$ jets samples. The uncertainty from the bad lepton method is larger than that of the method which is based on the di-jet method. The bad lepton method is biased by contamination of good leptons into the bad lepton sample, which is evident in the bad lepton to good lepton ratio in Figure 7.22 with a peak around $100 \mathrm{GeV}$ and in Figure 7.21 with the spikes near $\eta$ of 1.5. Since the total number of background events are similar in number, when the channels are combined, because there is no contaminiation issue in the di-jet method, and because the di-jet method has better statistical uncertainty, the background estimate 


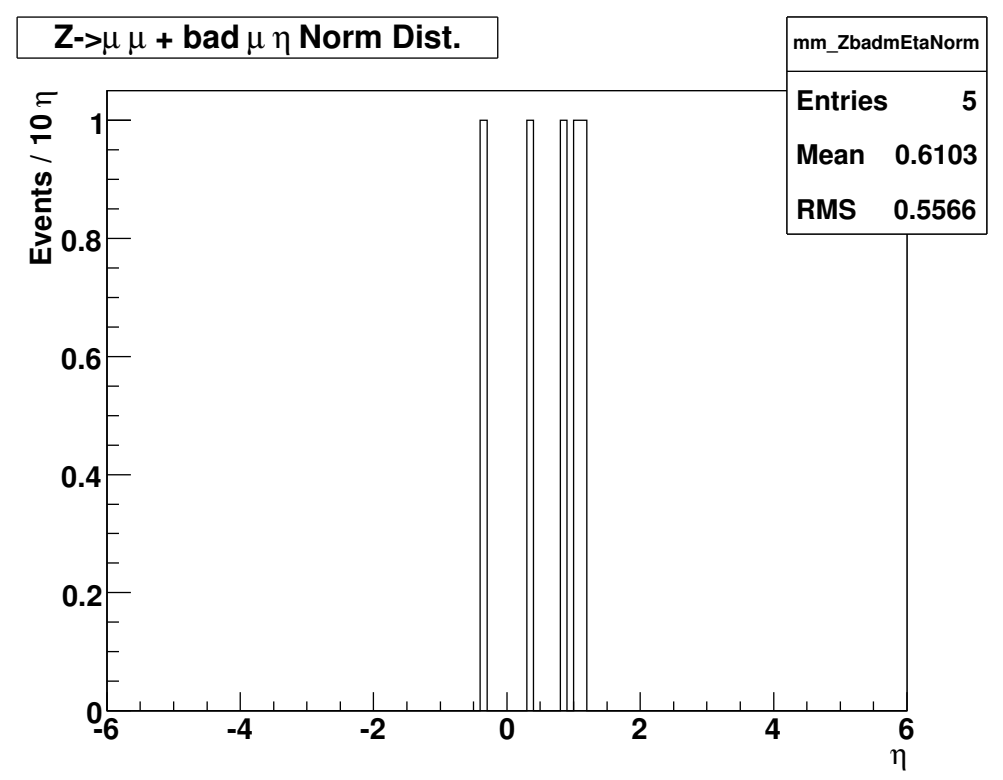

Figure 7.31: Normalization sample of $\mu \mu+$ bad $\mu$ events distributed in detector $\eta$.

based on the jet method is used in the final analysis.

\subsection{3 $\quad Z_{\gamma}$ Background}

$Z \gamma$ events can present a background to $W Z$ through either $\gamma$ conversion with a matched track or a random track overlap. Thus $Z \gamma$ can only enter into $\mu \mu e$ and eee final states. To estimate this background we apply our selection criteria to SM $\ell \ell \gamma$ samples provided by the $Z \gamma$ analysis group. [63]. These samples were produced using the Baur $Z \gamma$ generator with a cross section of $13.1 \pm 0.66 \mathrm{pb}$. This cross section is associated with the generator level cuts of $\Delta R(\gamma, \ell)>0.4$ and $E_{T}(\gamma)>3.0 \mathrm{GeV}$. The photon in these samples are treated as the non- $Z$ boson lepton. After applying the full selection criteria, the events are then multiplied by the efficiency for the photon to pass the electron likelihood criteria used in this analysis, $4.2 \pm 1.5 \%$ [64]. This efficiency is determined in data from $Z_{\gamma}$ final state radiation $\ell \ell \gamma$ events. When looking at the three body mass region around the $Z$ pole there is virtually no background contamination and therefore provides a pure sample of photons in which efficiencies of this nature can be measured. Please see [64] for 


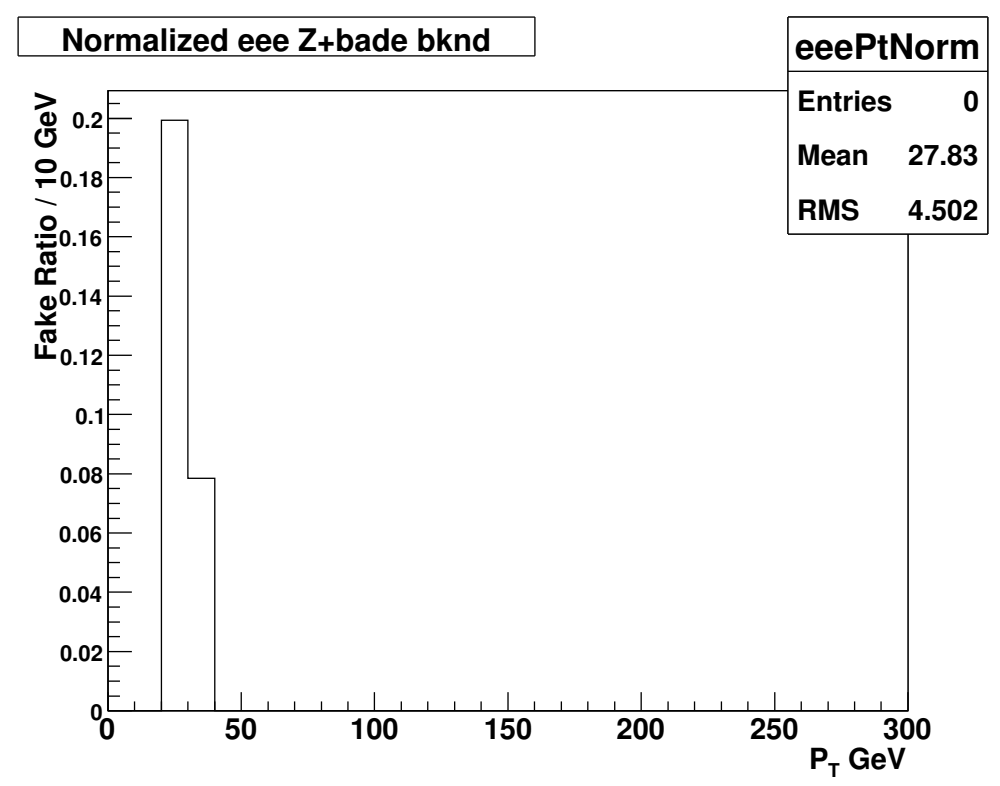

Figure 7.32: Normalized background estimate for the eee channel distributed in $p_{T}$. This distribution yields $0.278 \pm 0.133$ events. This distribution is determined by multiplying the distribution in Fig. 7.20 by the distribution in Fig. 7.24.

the full details of measuring efficiencies using this method. The input histograms to the efficiency are shown in Figure 7.40 where the $Z \gamma$ events that failed the electron likelihood requirement are plotted in red and the events that passed the electron likelihood are plotted in black. In both samples, a $15 \mathrm{GeV}$ cut is applied to the photon. As a cross check to this efficiency we take $\mathrm{D} \emptyset$ Geant simulated $\mu \mu+\gamma$ pythia generated events and find the ratio of detector level electrons, which pass our selection criteria, to generated photons. Since there are no electrons in the generation, it is assumed that the detector level electrons arise from the detector mis-identifying the photons as electrons. This gives a ratio of $6.0 \pm 0.3$ (stat.) \pm 2.9 (syst.)\%. The ratio plotted as a function of $E_{T}$ is shown in Figure 7.41. The results of the background estimation are shown in Table 7.8. The expected number of $Z \gamma$ events in the signal sample are $0.35 \pm 0.01$ in eee channel, and $1.01 \pm 0.41$ events in the $\mu \mu e$ channel. To check the normalization of our $Z \gamma$ estimate we compare the number of events we select in the sample region that fails our $\mathbb{E}_{T}$ cut, 


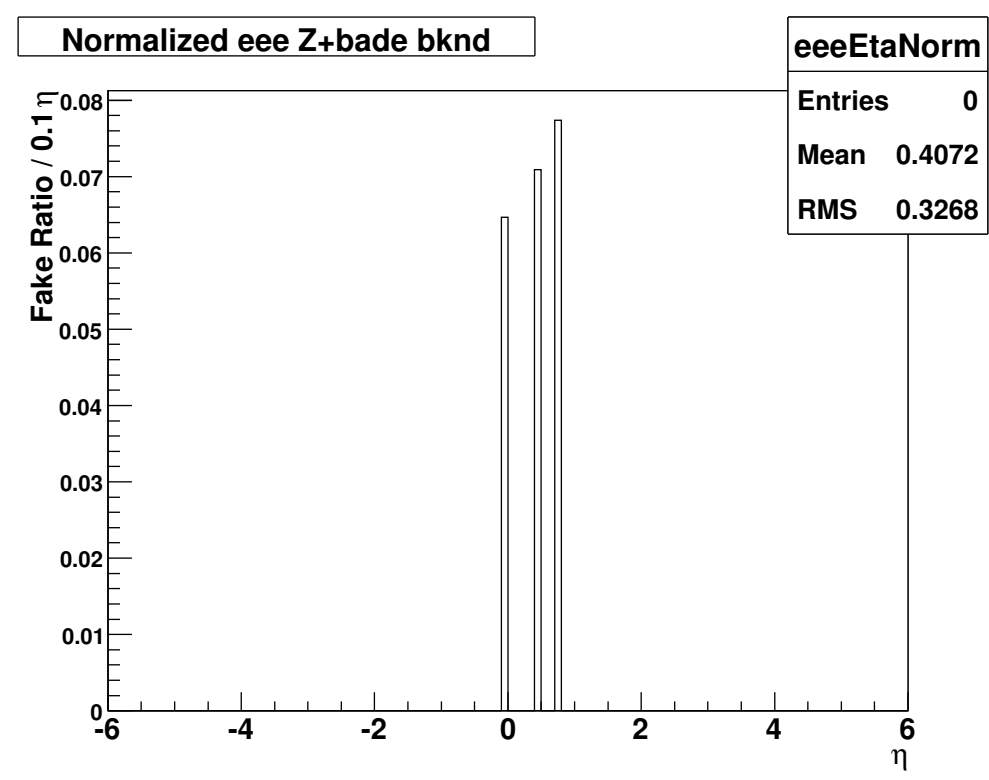

Figure 7.33: Normalized background estimate for the eee channel distributed in detector $\eta$. This distribution yields $0.213 \pm 0.115$ events. This distribution is determined by multiplying the distribution in Fig. 7.21 by the distribution in Fig. 7.25

and has no invariant mass cuts applied. We expect this sample to be dominated by final state radiation $Z+\gamma$ events where the $\gamma$ fakes an electron. The results of the study are presented in Appendix B, and agree well.

We use the equation

$$
N_{\text {background }}=\frac{\sigma_{\ell \ell \gamma}}{N_{\text {generated }}} \times \mathcal{L}_{\text {data }} \times N_{\text {selected }} \times f_{\gamma \rightarrow e}
$$

to calculate the $Z \gamma$ contribution to the background, where $\sigma_{\ell \ell \gamma}$ is the generated cross section, $N_{\text {generated }}$ is the number of $Z \gamma$ events generated, $\mathcal{L}_{\text {data }}$ is the equivalent luminosity in the data, $N_{\text {selected }}$ is the number of $Z \gamma \mathrm{MC}$ events that passed the event selection, and $f_{\gamma \rightarrow e}$ is the rate that a photon will be misidentified as an electron.

\subsection{4 $Z Z$ Background}

$Z Z$ events could also become background to our $W Z$ signal. In this case, the $Z$ s decay into a pair of leptons each, either electrons or muons. One of the four leptons can be lost 


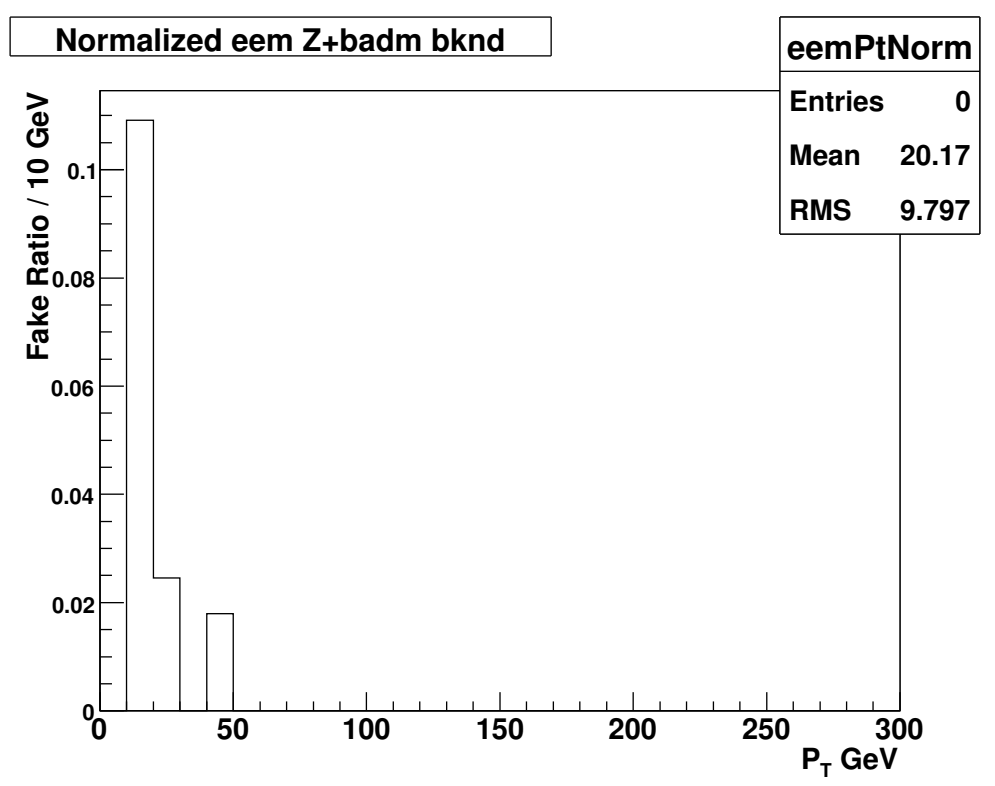

Figure 7.34: Normalized background estimate for the ee $\mu$ channel distributed in $p_{T}$. This distribution yields $0.152 \pm 0.044$ events. This distribution is determined by multiplying the distribution in Fig. 7.22 by the distribution in Fig. 7.26

in the detector, such as in the gap between detector modules and becomes $\mathbb{E}_{T}$ when reconstructed.

We estimate $Z Z$ background by using the following MC samples: MC request-id's 30835, 30836 and 30838. These MC samples are of on-shell $Z$ boson pairs. To estimate the contribution from $Z+$ Drell-Yan, we compare the on-shell sample with a sample of $Z+$ Drell-Yan at the generator level. From this study we estimate that the $Z+$ $Z / \gamma^{*}$ is approximately $10 \%$ of the on-shell $Z Z$ and will contribute that much more to the backgrounds. ${ }^{6}$ To properly normalize our MC we use the equation

$$
N_{\text {background }}=\frac{\sigma_{Z Z \rightarrow \ell \ell \ell^{\prime} \ell^{\prime}}}{N_{\text {generated }}} \times \mathcal{L}_{\text {data }} \times N_{\text {selected }}
$$

to estimate the $Z Z$ background contribution in our selection sample, where $\sigma_{Z Z \rightarrow \ell \ell \ell^{\prime} \ell^{\prime}}$ is the cross section for $Z Z$ to decay to four leptons, $1.42 \pm 0.08 \mathrm{pb}$ [23], $N_{\text {generated }}$ is the number of $Z Z$ events generated, $\mathcal{L}_{\text {data }}$ is the equivalent data luminosity and $N_{\text {selected }}$ is

\footnotetext{
${ }^{6}$ See the Section on Drell-Yan for a more detailed description or the $Z+Z / \gamma^{*}$ estimate.
} 


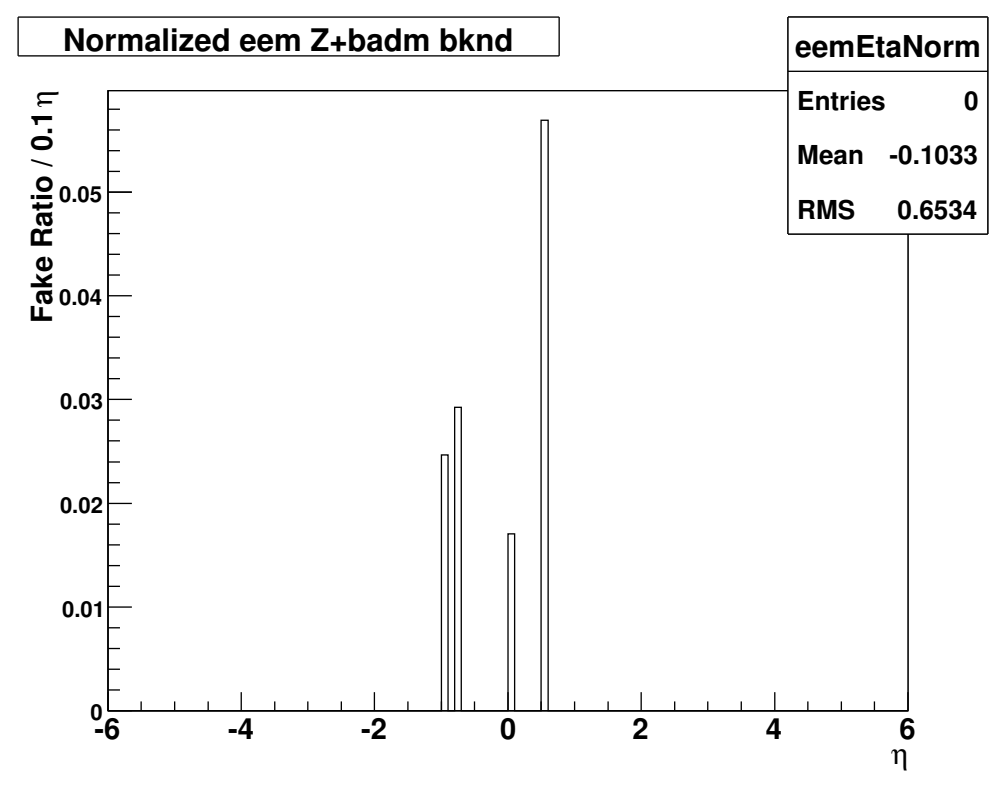

Figure 7.35: Normalized background estimate for the ee $\mu$ channel distributed in detector $\eta$. This distribution yields $0.128 \pm 0.055$ events. This distribution is determined by multiplying the distribution in Fig. 7.23 by the distribution in Fig. 7.27

the number of $Z Z \rightarrow \ell \ell \ell \ell \mathrm{MC}$ that passed the selection criteria. In the MC samples there were $26000 Z Z \rightarrow$ eeee, $25000 Z Z \rightarrow e е \mu \mu$ and $25250 Z Z \rightarrow \mu \mu \mu \mu$ events generated. We found 982 events in the eee channel, 3857 events in the ee $\mu$ channel, 1727 events in the $\mu \mu e$ channel and 7402 events in the $\mu \mu \mu$ channel. The $Z Z$ background contribution is summarized in Table 7.9.

\subsection{5 $t \bar{t}$ Background}

The dilepton decay of $t \bar{t}$ events can pass all of the event selection cuts and become fake candidates. In this scenario, a t $t \bar{t}$ event decays into $W \bar{W} b \bar{b}$. The $W$ 's further decay into leptons and b's semi-leptonic decay products have electrons or muons among hadronic daughters.

To estimate the background from $t \bar{t}$ events, MC samples are used. The request-ID's are 27901 and 27902. These contain $107750 t \bar{t} \rightarrow b \bar{b}+l l^{\prime}+E_{T}$ events. The events from request 27901 were generated with top quark mass of $170 \mathrm{GeV}$, and the events from request 27902 


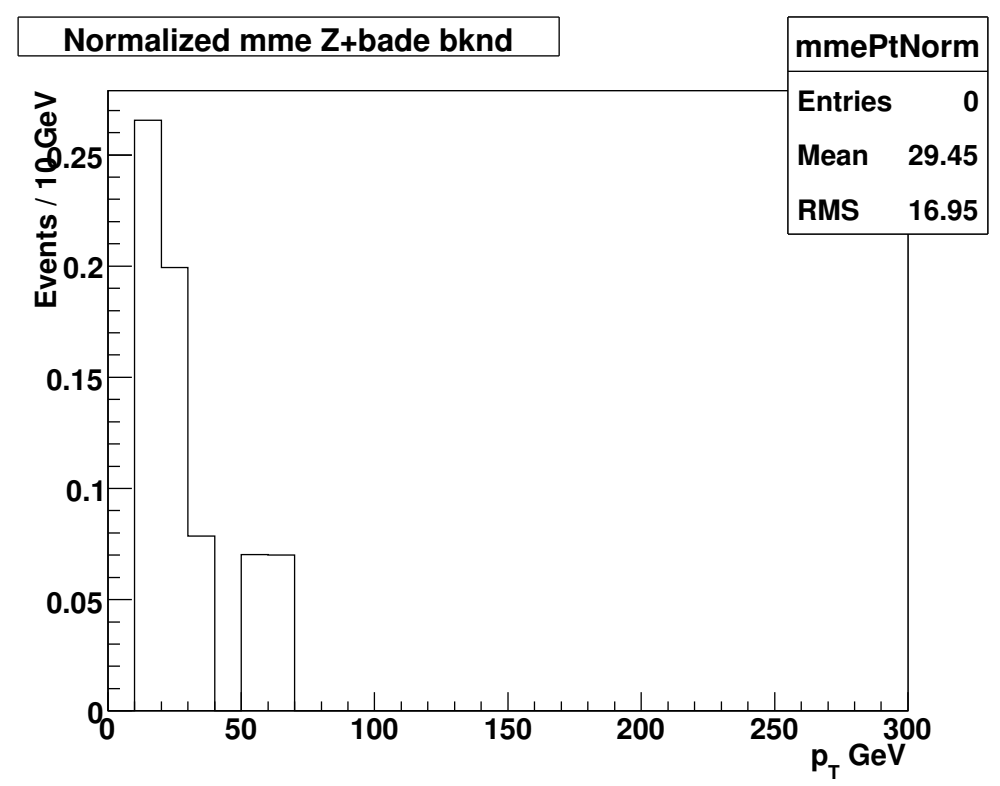

Figure 7.36: Normalized background estimate for the $\mu \mu e$ channel distributed in $p_{T}$. This distribution yields $0.684 \pm 0.288$ events. This distribution is determined by multiplying the distribution in Fig. 7.20 by the distribution in Fig. 7.28

were generated with a top quark mass of $180 \mathrm{GeV}$.

We use our event selection code to select trilepton events from this $t \bar{t} \mathrm{MC}$ sample data and found 2 eee events, 1 ee $\mu$ event and $1 \mu \mu e$ event. No $\mu \mu \mu$ events were found. The equivalent luminosity of the MC sample is $107750 /((4 / 9) * 0.11 * 0.11 * 7.3)=274 \mathrm{fb}^{-1}$. The fake events are normalized to data. We use the equation

$$
N_{\text {background }}=\frac{\sigma_{t \bar{t} \rightarrow \ell \bar{\ell}^{\prime}+j j}}{N_{\text {generated }}} \times \mathcal{L}_{\text {data }} \times N_{\text {selected }}
$$

to estimate the $t \bar{t}$ background contribution. Here $\sigma_{t \bar{t}}$ is the $t \bar{t}$ cross section of $7.30 \pm 0.88$ $\mathrm{pb}, N_{\text {generated }}$ is the number of $t \bar{t}$ events generated, $\mathcal{L}_{\text {data }}$ is the equivalent data luminosity and $N_{\text {selected }}$ is the number of selected $t \bar{t} \mathrm{MC}$ events.

The net $t \bar{t}$ contribution to the background is found to be $0.03 \pm 0.01$ events. 


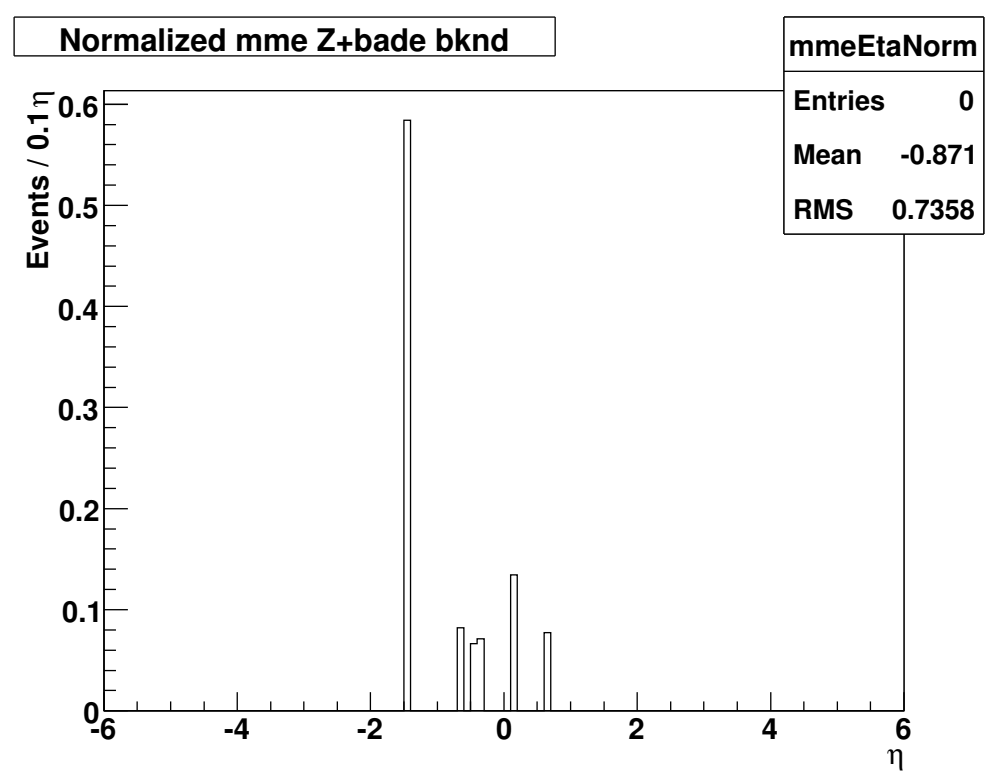

Figure 7.37: Normalized background estimate for the $\mu \mu e$ channel distributed in detector $\eta$. This distribution yields $1.016 \pm 0.428$ events. This distribution is determined by multiplying the distribution in Fig. 7.21 by the distribution in Fig. 7.29

\subsubsection{Drell-Yan Background}

The final source of background discussed is production of the diboson final state $W+$ $\gamma^{*}$. The estimation of this background is made difficult because PYTHIA only generates on-shell $W Z$ and does not include the $W \gamma^{*}$ or interference. Therefore we estimate this by generating $Z Z$ and $Z / \gamma^{*}+Z / \gamma^{*}$ and determining what fraction is off-shell. We use this fraction with the SM prediction for on-shell production to estimate this background. To assess a reasonable uncertainty on this we compare this result to the fractions from onshell $Z$ and $Z / \gamma^{*}$. It is estimated that $10.4 \pm 4.6 \%, 8.2 \pm 4.6 \% 9.1 \pm 3.7 \%$, and $14.8 \pm 3.7 \%$ of our estimated eee, ee $\mu, \mu \mu e$ and $\mu \mu \mu$ events, respectively, are actually from Drell-Yan. These estimates include the possibility for a mis-identified $Z$ boson that accepts DrellYan outside the mass window cuts. As mentioned above, this Drell-Yan contribution estimation is also applied to the $Z Z$ background where the $\mathrm{MC}$ was of on-shell $Z Z$ only. 


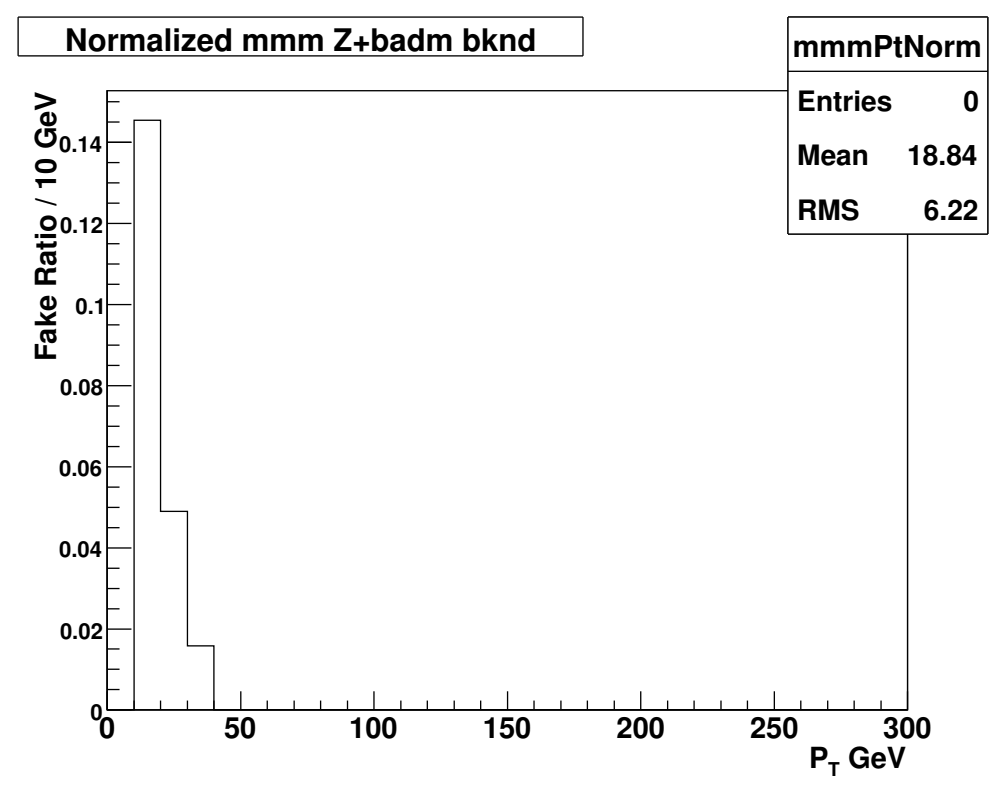

Figure 7.38: Normalized background estimate for the $\mu \mu \mu$ channel distributed in $p_{T}$. This distribution yields $0.196 \pm 0.092$ events. This distribution is determined by multiplying the distribution in Fig. 7.22 by the distribution in Fig. 7.30

\subsubsection{Total Background}

The background estimation is summarized in Table 7.12. The total background is estimated to be $4.5 \pm 0.6$.

\subsection{Systematics}

Since the number of $W Z$ candidate events is still quite small, the statistical uncertainty dominates this analysis. However, for completeness, we discuss the various systematic contributions to the total uncertainty. To determine these contributions a rigorous error analysis was performed on the number of expected events from both the signal and the background. Table 7.14 summarizes the dominant sources of systematic error on the number of expected events from signal. Table 7.15 summarizes the dominant sources of systematic uncertainty on the number of expected events of the backgrounds. The values of the uncertainties in both tables are given in number of events as that is what is used in the cross section likelihood determination. 


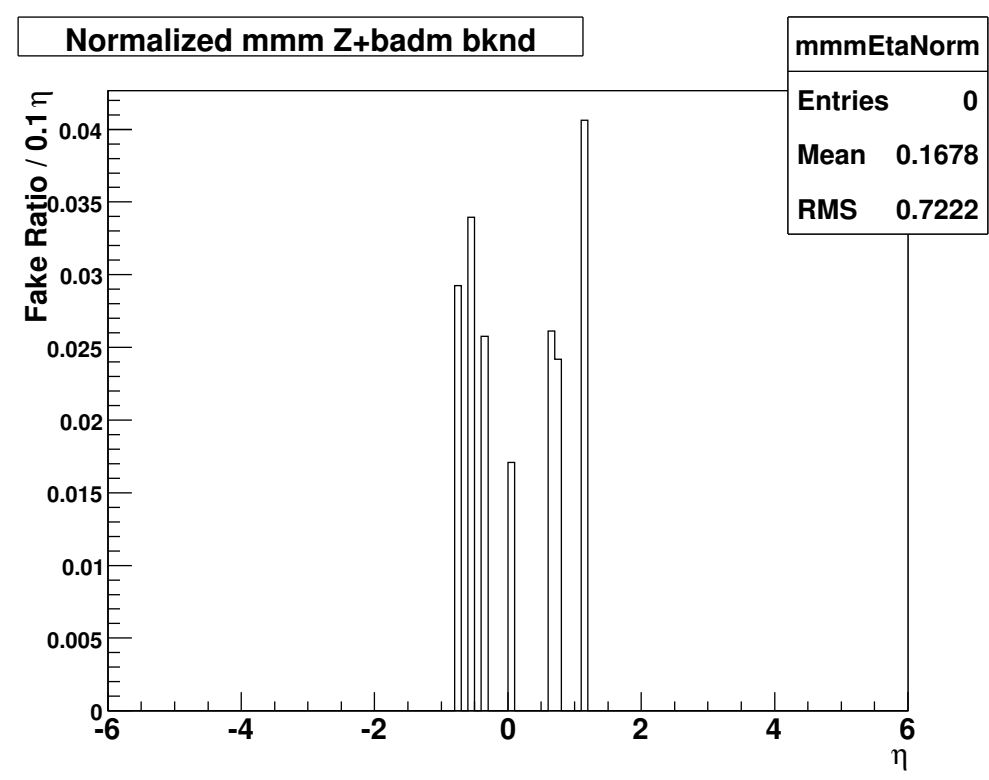

Figure 7.39: Normalized background estimate for the $\mu \mu \mu$ channel distributed in detector $\eta$. This distribution yields $0.197 \pm 0.093$ events. This distribution is determined by multiplying the distribution in Fig. 7.23 by the distribution in Fig. 7.31

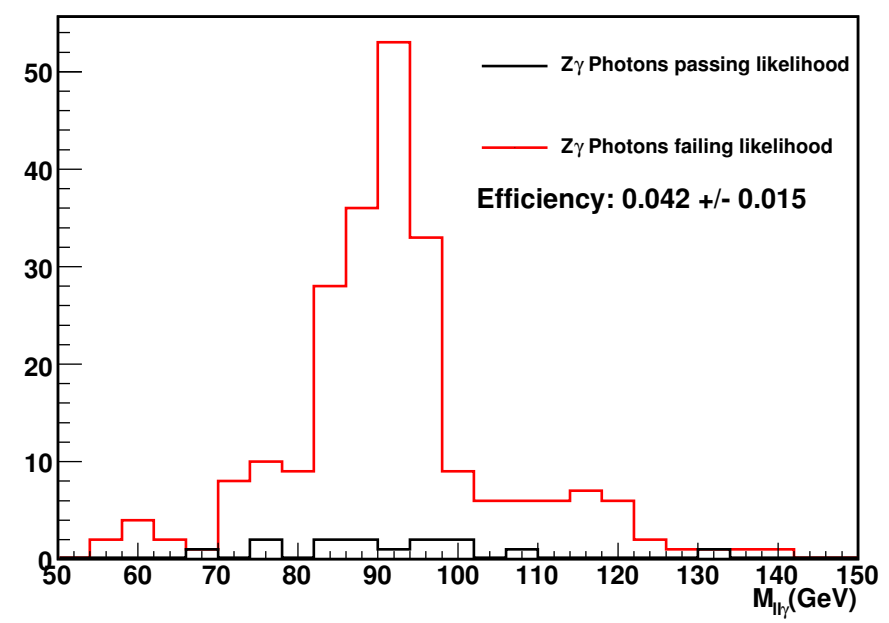

Figure 7.40: Shown are the three body masses of the $Z \gamma$ events used by the photon ID group. The black histogram are the events where the $\gamma$ passed the electron likelihood criteria. The red histogram are the events where the $\gamma$ failed the electron likelihood criteria. The photons in these samples also passed a threshold cut of $15 \mathrm{GeV}$. The resulting efficiency is $0.042 \pm 0.015$. The uncertainty is the statistical uncertainty.

In Table $7.15 f_{e}$ and $f_{m}$ refer to the uncertainties on the rates at which a jet is misidentified as an $e$ or a $\mu$. The QCD source listed is the uncertainty derived from the various QCD normalization samples. For the eee and $\mu \mu e$ channels the largest contribution 
Table 7.7: Shown are the background estimates from QCD using jets and bad leptons. The previous estimate used jets as a way to estimate the QCD contribution to the $W Z \rightarrow \ell \nu \ell \ell$ final state. The cross check uses bad leptons in jet triggered events to perform the same estimate. The uncertainties for the previous estimate are the combined statistical and systematic uncertainties. The uncertainties on the cross check values are the statistical and systematic uncertainties respectively. The statistical uncertainties on the cross check values are approximated as Poisson errors. The systematics uncertainties on the cross check is estimated by taking the difference of the normalized backgrounds distributed in $p_{T}$ to those distributed in $\eta$.

\begin{tabular}{|c|c|c|}
\hline Channel & $\begin{array}{c}\text { Di-Jet Method } \\
\text { (events) }\end{array}$ & $\begin{array}{c}\text { Bad Lepton Method } \\
\text { (events) }\end{array}$ \\
\hline$e e e$ & $0.54 \pm 0.04$ & $0.25 \pm 0.18 \pm 0.03$ \\
$e e \mu$ & $0.08 \pm 0.02$ & $0.14 \pm 0.07 \pm 0.01$ \\
$\mu \mu e$ & $0.58 \pm 0.05$ & $0.85 \pm 0.52 \pm 0.17$ \\
$\mu \mu \mu$ & $0.09 \pm 0.03$ & $0.20 \pm 0.13 \pm 0.01$ \\
\hline Total & $1.29 \pm 0.10$ & $1.44 \pm 0.57 \pm 0.17$ \\
\hline
\end{tabular}

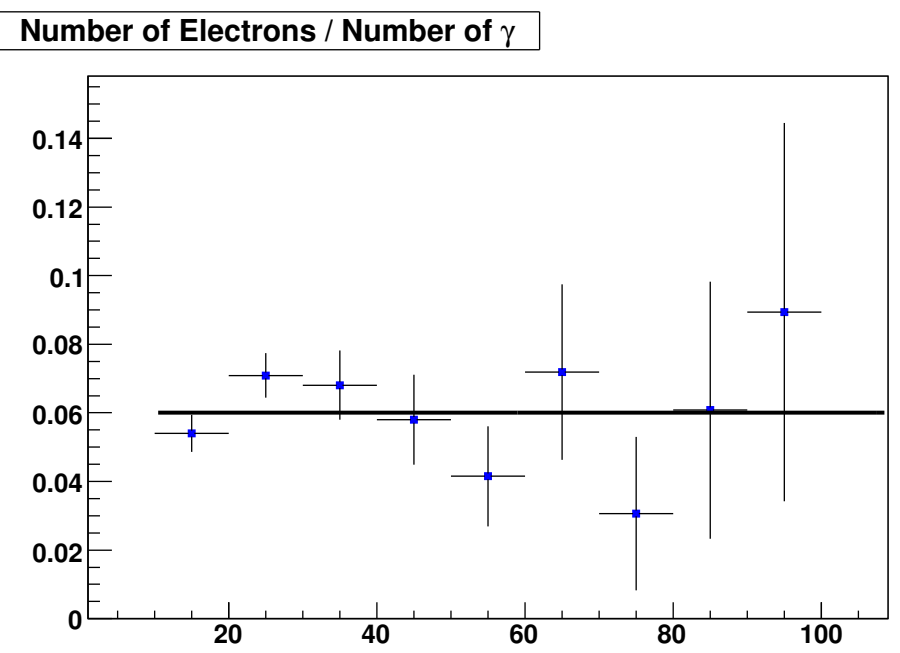

Figure 7.41: Presented is the ratio of selected electrons to generated photons in a sample of $\mu \mu+\gamma$ initial state radiation pythia $\mathrm{MC}$ that has been simulated using the $\mathrm{D} \varnothing$ Geant detector simulation. The error bars are statistical uncertainties. The line is the average ratio, $0.06 \pm 0.003$. A systematic error on the ratio is determined by the highest and lowest bin in the sample resulting in an uncertainty of 0.029 .

Table 7.8: The $Z \gamma$ background is estimated using MC events from SM PYTHIA. The backgrounds are listed in the table.

\begin{tabular}{|c|c|c|c|}
\hline Channel & $\begin{array}{c}\text { Number } \\
\text { Generated }\end{array}$ & $\begin{array}{c}Z \gamma \\
\text { events passed }\end{array}$ & $\begin{array}{c}\text { Background } \\
\text { (events) }\end{array}$ \\
\hline eee & 259167 & 220 & $0.35 \pm 0.01$ \\
$\mu \mu e$ & 259167 & 722 & $1.01 \pm 0.41$ \\
\hline Total & 518334 & 32.4 & $1.36 \pm 0.54$ \\
\hline
\end{tabular}


Table 7.9: $Z Z$ background contribution. The uncertainties are the combined systematic and statistical uncertainties.

\begin{tabular}{|c|c|c|c|}
\hline \hline Channel & $\begin{array}{c}\text { Number } \\
\text { Generated }\end{array}$ & $\begin{array}{c}\text { Number } \\
\text { Accepted }\end{array}$ & $\begin{array}{c}\text { Background } \\
\text { (events) }\end{array}$ \\
eee & 26000 & 982 & $0.05 \pm 0.004$ \\
$e е \mu$ & 25000 & 3857 & $0.19 \pm 0.02$ \\
$\mu \mu e$ & 25000 & 1727 & $0.08 \pm 0.01$ \\
$\mu \mu \mu$ & 25250 & 7402 & $0.38 \pm 0.04$ \\
\hline Total & 76250 & 13968 & $0.70 \pm 0.08$ \\
\hline \hline
\end{tabular}

Table 7.10: $t \bar{t}$ background. The uncertainties are the combined systematic and statistical uncertainties.

\begin{tabular}{|c|c|c|c|}
\hline \hline Channel & $\begin{array}{c}\text { Number } \\
\text { Generated }\end{array}$ & $\begin{array}{c}\text { Number } \\
\text { Accepted }\end{array}$ & $\begin{array}{c}\text { Background } \\
\text { (events) }\end{array}$ \\
$e e e$ & 107750 & 2 & $0.01 \pm 0.01$ \\
$e е \mu$ & 107750 & 1 & $0.01 \pm 0.01$ \\
$\mu \mu e$ & 107750 & 1 & $0.01 \pm 0.01$ \\
$\mu \mu \mu$ & 107750 & 0 & $<0.01$ \\
\hline Total & 107750 & 4 & $0.03 \pm 0.01$ \\
\hline \hline
\end{tabular}

arise from the $Z \gamma$ acceptance. Most other leading contributions come from the QCD backgrounds.

Table 7.11: Drell Yan

\begin{tabular}{|c|c|}
\hline \hline Channel & Background (events) \\
$e e e$ & $0.24 \pm 0.01$ \\
$e е \mu$ & $0.17 \pm 0.01$ \\
$\mu \mu e$ & $0.21 \pm 0.01$ \\
$\mu \mu \mu$ & $0.38 \pm 0.01$ \\
\hline Total & $0.99 \pm 0.02$ \\
\hline \hline
\end{tabular}


Table 7.12: Estimated background broken down by signal decay channel. The uncertainty on the backgrounds are the combined statistical and systematic errors.

\begin{tabular}{|c|c|c|}
\hline Channel & Source & $\begin{array}{c}\text { Estimated background } \\
\text { Events } \pm \text { (Stat. }+ \text { Syst.) }\end{array}$ \\
\hline eee & $\begin{array}{c}Z \rightarrow e e+\text { jets } \\
Z Z \\
Z \gamma \\
t \bar{t} \\
\text { Drell-Yan }\end{array}$ & $\begin{array}{c}0.54 \pm 0.04 \\
0.05 \pm 0.004 \\
0.36 \pm 0.01 \\
0.01 \pm 0.01 \\
0.24 \pm 0.01\end{array}$ \\
\hline subtotal & & $1.19 \pm 0.14$ \\
\hline$e e \mu$ & $\begin{array}{c}e+\mu+\text { jets } \\
Z \rightarrow e e+\text { jets } \\
Z Z \\
t \bar{t} \\
\text { Drell-Yan }\end{array}$ & $\begin{array}{c}0.01 \pm 0.002 \\
0.08 \pm 0.02 \\
0.19 \pm 0.02 \\
0.01 \pm 0.01 \\
0.18 \pm 0.01\end{array}$ \\
\hline subtotal & & $0.46 \pm 0.03$ \\
\hline$\mu \mu e$ & $\begin{array}{c}Z \rightarrow \mu \mu+\text { jets } \\
e+\mu+\text { jets } \\
Z Z \\
Z \gamma \\
t \bar{t} \\
\text { Drell-Yan }\end{array}$ & $\begin{array}{l}0.58 \pm 0.05 \\
\quad<0.01 \\
0.08 \pm 0.01 \\
1.01 \pm 0.41 \\
0.01 \pm 0.01 \\
0.21 \pm 0.01\end{array}$ \\
\hline subtotal & & $1.96 \pm 0.42$ \\
\hline$\mu \mu \mu$ & $\begin{array}{c}Z \rightarrow \mu \mu+\text { jets } \\
Z Z \\
t \bar{t} \\
\text { Drell-Yan }\end{array}$ & $\begin{array}{l}0.09 \pm 0.03 \\
0.38 \pm 0.04 \\
\quad<0.01 \\
0.38 \pm 0.01\end{array}$ \\
\hline subtotal & & $0.86 \pm 0.06$ \\
\hline Total & & $4.47 \pm 0.57$ \\
\hline
\end{tabular}

Table 7.13: Shown are the backgrounds broken down by source.

\begin{tabular}{|c|c|}
\hline Source & $\begin{array}{c}\text { Estimated Background } \\
\text { Events } \pm \text { (Syst. }+ \text { Stat. })\end{array}$ \\
\hline QCD & $1.32 \pm 0.10$ \\
Drell-Yan & $0.99 \pm 0.02$ \\
ZZ & $0.70 \pm 0.08$ \\
Z $\gamma$ & $1.44 \pm 0.54$ \\
$t \bar{t}$ & $0.03 \pm 0.01$ \\
\hline Total & $4.47 \pm 0.57$ \\
\hline
\end{tabular}


Table 7.14: Presented is a breakdown of the systematics contributing the uncertainty on the number of signal events expected. The first source, cross section, in the table is broken down into its PDF and scale sources.

\begin{tabular}{|c|c|c|}
\hline \hline Source & uncertainty (events) & uncertainty (\%) \\
\hline \hline Cross Section $(W Z)$ & 0.624 & 6.8 \\
pdf & 0.298 & 3.2 \\
scale & 0.548 & 6.0 \\
\hline luminosity & 0.596 & 6.5 \\
\hline
\end{tabular}

Table 7.15: Presented are the dominant systematics, broken down by channel, that contribute to the number of background events, and ultimately contribute to the systematic uncertainty in the cross section determination. The QCD source is the contribution from the QCD normalization samples. The $f_{e}$ and $f_{m}$ are the contributions from the lepton fake rates from jets. The $\epsilon_{e}$ and $\epsilon_{\mu}$ are the contributions from the lepton ID efficiencies.

\begin{tabular}{|c|c|c|c|}
\hline Channel & Source & uncertainty (events) & uncertainty (\%) \\
\hline eee channel & $\begin{array}{c}\epsilon_{e} \\
\mathrm{QCD} \\
f_{e} \\
Z \gamma \text { Acceptance } \\
\sigma_{Z \gamma} \\
\gamma \rightarrow e \\
Z Z \text { Acceptance } \\
\sigma_{Z Z} \\
\text { Drell-Yan } \\
\text { Luminosity }\end{array}$ & $\begin{array}{l}0.007 \\
0.013 \\
0.042 \\
0.023 \\
0.043 \\
0.159 \\
0.001 \\
0.003 \\
0.011 \\
0.015\end{array}$ & $\begin{array}{c}0.2 \\
0.3 \\
0.9 \\
0.5 \\
1.0 \\
3.6 \\
0.02 \\
0.1 \\
0.2 \\
0.3\end{array}$ \\
\hline$e e \mu$ channel & $\begin{array}{c}\epsilon_{e} \\
\epsilon_{\mu} \\
\mathrm{QCD} \\
f_{m} \\
f_{e} \\
Z Z \text { Acceptance } \\
\sigma_{Z Z} \\
\text { Drell-Yan } \\
\text { Luminosity }\end{array}$ & $\begin{array}{l}0.004 \\
0.007 \\
0.003 \\
0.023 \\
0.001 \\
0.003 \\
0.011 \\
0.008 \\
0.013\end{array}$ & $\begin{array}{c}0.1 \\
0.2 \\
0.1 \\
0.5 \\
0.02 \\
0.1 \\
0.2 \\
0.2 \\
0.3\end{array}$ \\
\hline$\mu \mu e$ channel & $\begin{array}{c}\epsilon_{e} \\
\epsilon_{\mu} \\
\mathrm{QCD} \\
f_{m} \\
f_{e} \\
Z \gamma \text { Acceptance } \\
\sigma_{Z \gamma} \\
\gamma \rightarrow e \\
Z Z \text { Acceptance } \\
\sigma_{Z Z} \\
\text { Drell-Yan } \\
\text { Luminosity }\end{array}$ & $\begin{array}{l}0.003 \\
0.021 \\
0.013 \\
0.001 \\
0.045 \\
0.040 \\
0.054 \\
0.387 \\
0.002 \\
0.004 \\
0.008 \\
0.020\end{array}$ & $\begin{array}{c}0.1 \\
0.5 \\
0.3 \\
0.02 \\
1.0 \\
0.9 \\
1.2 \\
8.7 \\
0.04 \\
0.1 \\
0.2 \\
0.4\end{array}$ \\
\hline$\mu \mu \mu$ channel & $\begin{array}{c}\epsilon_{\mu} \\
\mathrm{QCD} \\
f_{m} \\
Z Z \text { Acceptance } \\
\sigma_{Z Z} \\
\text { Drell-Yan } \\
\text { Luminosity }\end{array}$ & $\begin{array}{l}0.040 \\
0.002 \\
0.026 \\
0.004 \\
0.021 \\
0.014 \\
0.025\end{array}$ & $\begin{array}{c}0.9 \\
0.04 \\
0.6 \\
0.1 \\
0.5 \\
0.3 \\
0.6\end{array}$ \\
\hline
\end{tabular}




\section{CHAPTER 8}

\section{Cross Section Measurement and WZ Gauge Coupling Measurement}

\subsection{Cross section}

The $W Z$ cross section is thus calculated to be $\sigma_{W Z}=2.7_{-1.3}^{+1.7}$, using a likelihood method based on the number of expected events. Using Poisson statistics, we calculate the probability for the number of observed events to be from our expected $W Z$ production plus the background as determined by

$$
p(n ; \nu)=\ln \frac{\nu^{n} e^{-\nu}}{n !}
$$

where $\nu$ is the predicted number of events from the expected signal plus background. We define $\nu$ as

$$
\nu=\nu_{s}+\nu_{b}
$$

where $\nu_{s}$ is a function of the cross section,

$$
\nu_{s}=\sigma_{W Z}^{\prime} \times \mathcal{L} \times \mathcal{A}
$$

Where $\sigma_{W Z}^{\prime}$ is the cross section in which we determine the likelihood, $\mathcal{L}$ is the luminosity of channel, and $\mathcal{A}$ is the acceptance times efficiency of the channel. The $\log$ 
likelihood takes into account the uncertainties in the luminosities, acceptances and backgrounds by integrating over them as Gaussian uncertainties. The Gaussian uncertainties are determined by

$$
g_{i}\left(x_{i}, \sigma_{i}\right)=\frac{e^{-\frac{\left(x_{i}-\nu_{i}\right)^{2}}{2 \sigma_{i}^{2}}}}{\sqrt{2 \pi} \sigma_{i}}, \text { where } i=s, b \text { and } x_{i} \in\left[-3 \sigma_{i},+3 \sigma_{i}\right]
$$

The final log likelihood is determined by

$$
L=-\ln \left(\int_{-3 \sigma_{l}}^{3 \sigma_{l}} \int_{-3 \sigma_{b}}^{3 \sigma_{b}} \int_{-3 \sigma_{s}}^{3 \sigma_{s}} g_{l} g_{b} g_{s} \frac{\left(x_{l} x_{s} \nu_{s}+x_{b} \nu_{b}\right)^{n} e^{-\left(x_{l} x_{s} \nu_{s}+x_{b} \nu_{b}\right)}}{n !} d x_{s} d x_{b} d x_{l}\right)
$$

As a comparison, the theoretical prediction is $3.68 \pm 0.22$ (scale) \pm 0.12 (PDF) pb [23]. Since the number of candidate events is still low, in contrast we can form the likelihood for each channel as a function of the cross section, and then combine the likelihoods to provide a value and uncertainty on the measured cross section. The combined negative $\log$ likelihood is shown in Figure 8.1. The minimum of the negative log likelihood (corresponding to the most probable value for the cross section) is $2.7 \mathrm{pb}$. The uncertainty on this value is estimated by moving up 0.5 units of negative log likelihood, which yields an asymmetric error. The final cross section from the likelihood calculation is $\sigma_{W Z}=2.7_{-1.3}^{+1.7} \mathrm{pb}$

The significance for each channel individually, along with the total candidates is summarized in Table 8.1. The probability for the estimated background to fluctuate to the number of observed candidate events is calculated using the standard D $\varnothing$ prescription (a Gaussian background convoluted with a Poissonian fluctuation probability). Then the standard inverse error function prescription is used to transform this probability into a significance. 


\section{WZ Cross Section Likelihood Curve}

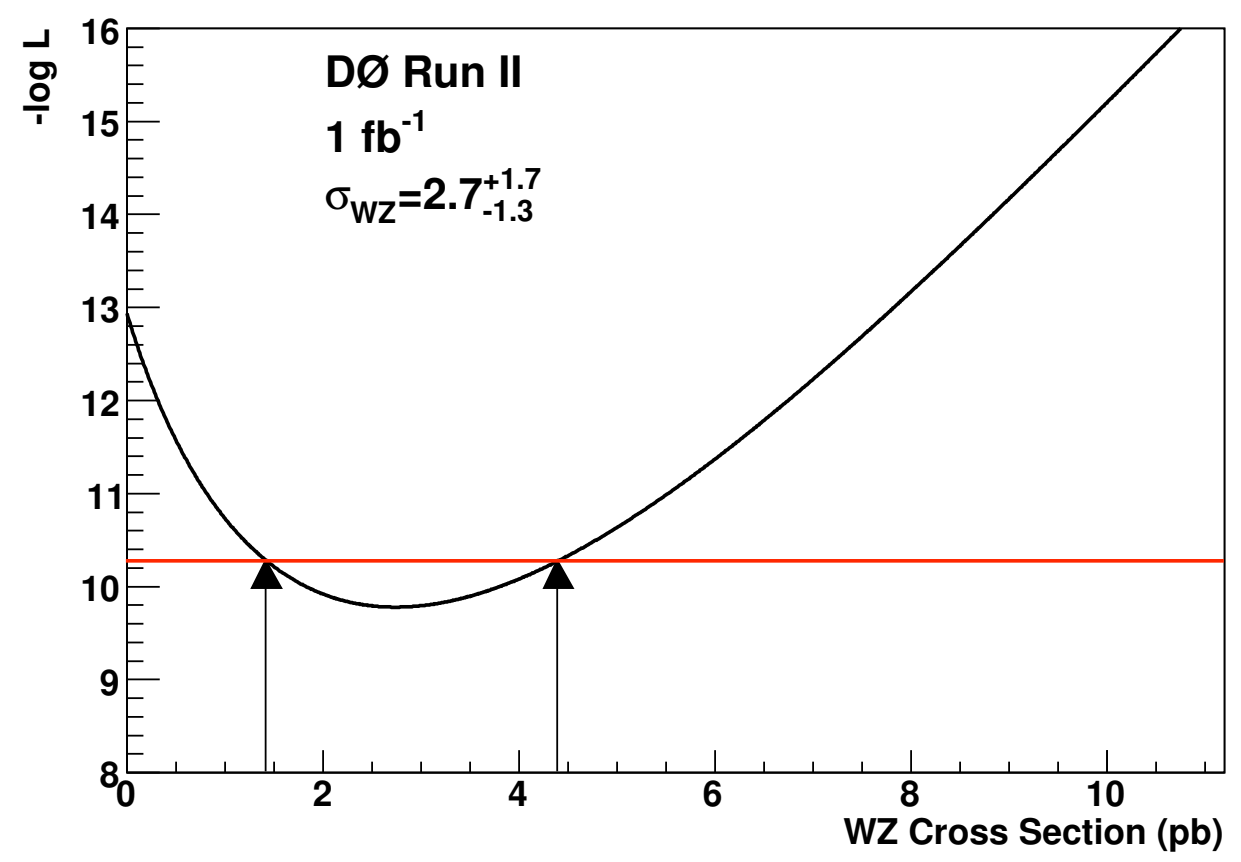

Figure 8.1: Combined negative log likelihood as a function of cross section. Arrows indicate the points 0.5 units of likelihood above the minimum.

Table 8.1: Summary of probability of background to fluctuate to the observed number of candidates, and significance by channel.

\begin{tabular}{|c|c|c|c|c|}
\hline \hline $\begin{array}{c}\text { Decay } \\
\text { Channel }\end{array}$ & Probability & $\begin{array}{c}\text { Significance } \\
(\sigma)\end{array}$ & $\begin{array}{c}\text { Total Number } \\
\text { of Events }\end{array}$ & $\begin{array}{c}\text { Background } \\
\text { Events }\end{array}$ \\
\hline$e e e$ & 0.333 & 0.431 & 2 & $1.19 \pm 0.14$ \\
\hline$e e \mu$ & 0.368 & 0.336 & 1 & $0.46 \pm 0.03$ \\
\hline$\mu \mu e$ & 0.0017 & 2.92 & 8 & $1.96 \pm 0.42$ \\
\hline$\mu \mu \mu$ & 0.213 & 0.796 & 2 & $0.86 \pm 0.06$ \\
\hline Total & 0.0012 & 3.0 & 13 & $4.47 \pm 0.57$ \\
\hline Expected & 0.0012 & 3.0 & 13.6 & $4.47 \pm 0.57$ \\
\hline
\end{tabular}




\section{CHAPTER 9}

\section{Conclusion}

\subsection{Conclusion}

The $\mathrm{D} \varnothing$ collaboration has found the first evidence of $W Z$ production through this thesis work. We expect to see $9.2 \pm 1.0$ signal events and $4.5 \pm 0.6$ background events. After searching in $\sim 1 \mathrm{fb}^{-1}$ of $p \bar{p}$ collisions we find 13 events that pass our $W Z$ selection criteria. The probability for $4.5 \pm 0.6$ background events to fluctuate to 13 or more events is $1.2 \times 10^{-3}$. This is a greater than $3.0 \sigma$ significance. With this significance the cross section of $W Z$ production at the Tevatron is measured to be $\sigma_{W Z}=2.7_{-1.3}^{+1.7} \mathrm{pb}$, which is consistent with the SM prediction. 
APPENDICES 


\section{APPENDIX A}

\section{Cut Flows}

These cut flows contain information from the analysis where a $20 \mathrm{GeV}$ threshold was applied to $\mathbb{E}_{T}$.

Table A.1: This table shows the cut flows for the data and signal MC. The cut flows start from the third lepton cut. The data also has the separation cut applied before the third lepton cut as this cut removes many events that contain two lepton that share the same track. The cause of this is speculated to be $\mu$ 's radiating bremsstrahlung radiation which gets reconstructed as an electron. This effect is not modeled well in the detector simulation therefore it would not show up in the MC samples.

\begin{tabular}{|c|c|c|c|c|c|c|}
\hline Cut & Data $Z \rightarrow e e$ & Data $Z \rightarrow \mu \mu$ & MC eee & MC ee $\mu$ & MC $\mu \mu e$ & MC $\mu \mu \mu$ \\
\hline \hline third lepton & 25 & 34 & $2.79 \pm 0.28$ & $2.43 \pm 0.26$ & $2.76 \pm 0.37$ & $3.00 \pm 0.45$ \\
$\mathbb{E}_{T}$ & 6 & 12 & $2.36 \pm 0.24$ & $2.14 \pm 0.23$ & $2.37 \pm 0.32$ & $2.66 \pm 0.39$ \\
separation & 6 & 12 & $2.34 \pm 0.24$ & $2.12 \pm 0.23$ & $2.35 \pm 0.32$ & $2.64 \pm 0.39$ \\
$V_{E_{T} \text { had }}$ & 5 & 12 & $2.27 \pm 0.23$ & $2.06 \pm 0.22$ & $2.29 \pm 0.31$ & $2.56 \pm 0.38$ \\
delta $z$ & 3 & 10 & $2.27 \pm 0.23$ & $2.06 \pm 0.22$ & $2.29 \pm 0.31$ & $2.56 \pm 0.38$ \\
\hline
\end{tabular}

Table A.2: This table shows the cut flows for the MC backgrounds.

\begin{tabular}{|c|c|c|c|c|c|c|}
\hline Cut & $Z Z \rightarrow e e e$ & $Z Z \rightarrow e e \mu$ & $Z Z \rightarrow \mu \mu e$ & $Z Z \rightarrow \mu \mu \mu$ & $Z \gamma \rightarrow e e e$ & $Z \gamma \rightarrow \mu \mu e$ \\
\hline \hline third lepton & $0.678 \pm 0.163$ & $0.428 \pm 0.082$ & $0.445 \pm 0.094$ & $0.675 \pm 0.138$ & $13.9 \pm 0.38$ & $14.9 \pm 6.05$ \\
$E_{T}$ & $0.078 \pm 0.009$ & $0.208 \pm 0.023$ & $0.103 \pm 0.015$ & $0.403 \pm 0.053$ & $0.35 \pm 0.01$ & $1.01 \pm 0.41$ \\
separation & $0.072 \pm 0.007$ & $0.203 \pm 0.022$ & $0.099 \pm 0.014$ & $0.398 \pm 0.051$ & $0.35 \pm 0.01$ & $1.01 \pm 0.41$ \\
$V_{E_{T} \text { Had }}$ & $0.046 \pm 0.004$ & $0.193 \pm 0.019$ & $0.079 \pm 0.010$ & $0.391 \pm 0.044$ & $0.35 \pm 0.01$ & $1.01 \pm 0.41$ \\
delta $z$ & $0.046 \pm 0.004$ & $0.193 \pm 0.019$ & $0.078 \pm 0.010$ & $0.381 \pm 0.043$ & $0.35 \pm 0.01$ & $1.01 \pm 0.41$ \\
\hline
\end{tabular}

Table A.3: This table shows the cut flows for the $t \bar{t}$ backgrounds.

\begin{tabular}{|c|c|c|c|c|}
\hline Cut & $t \bar{t} \rightarrow e e e$ & $t \bar{t} \rightarrow e e \mu$ & $t \bar{t} \rightarrow \mu \mu e$ & $t \bar{t} \rightarrow \mu \mu \mu$ \\
\hline \hline third lepton & $0.070 \pm 0.027$ & $0.061 \pm 0.023$ & $0.075 \pm 0.026$ & $0.092 \pm 0.031$ \\
$\mathbb{E}_{T}$ & $0.060 \pm 0.020$ & $0.061 \pm 0.021$ & $0.065 \pm 0.021$ & $0.092 \pm 0.031$ \\
separation & $0.022 \pm 0.011$ & $0.022 \pm 0.012$ & $0.015 \pm 0.009$ & $0.006 \pm 0.006$ \\
$V_{E_{T} \text { Had }}$ & $0.016 \pm 0.011$ & $0.006 \pm 0.006$ & $0.005 \pm 0.005$ & $0.006 \pm 0.006$ \\
delta $z$ & $0.011 \pm 0.011$ & $0.006 \pm 0.006$ & $0.005 \pm 0.005$ & $0.006 \pm 0.006$ \\
\hline
\end{tabular}


Table A.4: This table shows the cut flows for the Drell Yan backgrounds.

\begin{tabular}{|c|c|c|c|c|}
\hline Cut & $e e e$ & $e e \mu$ & $\mu \mu e$ & $\mu \mu \mu$ \\
\hline \hline third lepton & $0.290 \pm 0.013$ & $0.199 \pm 0.009$ & $0.251 \pm 0.009$ & $0.444 \pm 0.016$ \\
$E_{T}$ & $0.245 \pm 0.011$ & $0.175 \pm 0.008$ & $0.216 \pm 0.008$ & $0.394 \pm 0.015$ \\
separation & $0.243 \pm 0.011$ & $0.174 \pm 0.008$ & $0.214 \pm 0.008$ & $0.391 \pm 0.014$ \\
$V_{E_{T} \text { Had }}$ & $0.236 \pm 0.011$ & $0.169 \pm 0.008$ & $0.208 \pm 0.008$ & $0.377 \pm 0.014$ \\
delta $z$ & $0.236 \pm 0.011$ & $0.169 \pm 0.008$ & $0.208 \pm 0.008$ & $0.377 \pm 0.014$ \\
\hline
\end{tabular}

Table A.5: This table shows the cut flows for the QCD backgrounds.

\begin{tabular}{|c|c|c|c|c|c|c|}
\hline Cut & $Z \rightarrow e e+$ jets $\rightarrow e$ & $Z \rightarrow e e+$ jets $\rightarrow \mu$ & $e+\mu+$ jets $\rightarrow e$ & $Z \rightarrow \mu \mu+$ jets $\rightarrow e$ & $e+\mu+$ jets $\rightarrow \mu$ & $Z \rightarrow \mu \mu+$ jets $\rightarrow \mu$ \\
\hline \hline third lepton & $4.03 \pm 0.29$ & $0.664 \pm 0.142$ & $0.526 \pm 0.040$ & $4.55 \pm 0.33$ & $0.082 \pm 0.018$ & $0.736 \pm 0.157$ \\
$\mathscr{E}_{T}$ & $0.612 \pm 0.046$ & $0.096 \pm 0.024$ & $0.121 \pm 0.010$ & $1.23 \pm 0.09$ & $0.018 \pm 0.004$ \\
separation & $0.610 \pm 0.046$ & $0.095 \pm 0.024$ & $0.093 \pm 0.008$ & $1.22 \pm 0.09$ & $0.015 \pm 0.006$ \\
$V_{E_{T} \text { Had }}$ & $0.543 \pm 0.044$ & $0.084 \pm 0.024$ & $0.042 \pm 0.005$ & $0.579 \pm 0.047$ & $0.006 \pm 0.001$ & $0.189 \pm 0.040$ \\
delta $z$ & $0.543 \pm 0.044$ & $0.084 \pm 0.024$ & $0.014 \pm 0.006$ & $0.579 \pm 0.047$ & $0.004 \pm 0.001$ & $0.093 \pm 0.020$ \\
\hline
\end{tabular}




\section{APPENDIX B}

\section{Control Sample}

To cross check the results of this search; the same analysis was performed on events that failed the $M_{\ell \ell}$ cut. This analysis yields three candidates, with an expectation of $1.25 \pm 0.36$ events from signal and $0.61 \pm 0.12$ events from background. One of the candidate events, event 70487134 in run 195841, has a very large $\mathbb{E}_{T}$ of $935 \mathrm{GeV}$, and a dilepton invariant mass of $763 \mathrm{GeV} / \mathrm{c}^{2}$. The invariant mass versus $\not_{T}$ distribution is show in Fig.B.1. The $E_{T}$ distribution is shown in Fig.B.2

Another control sample would be in the region of the invariant mass versus $\mathbb{E}_{T}$ space for events that fail the $\mathbb{E}_{T}$ cut. When we look at this region we find the data is dominated by $Z \gamma$ events mostly from final state radiation. This is seen in the invariant mass plot of this region shown in Figure B.4 


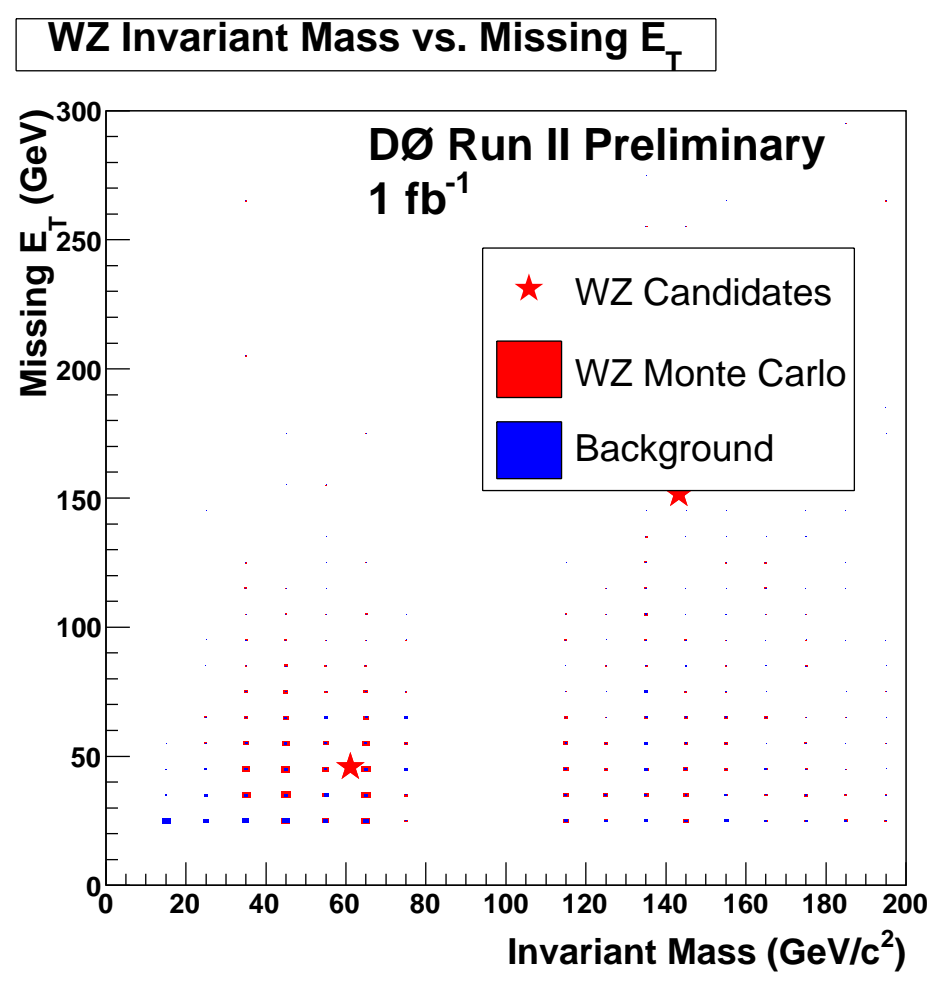

Figure B.1: The $\mathscr{E}_{T}$ versus invariant mass distributions of the sample that did not pass the $M_{\ell \ell}$ cuts.

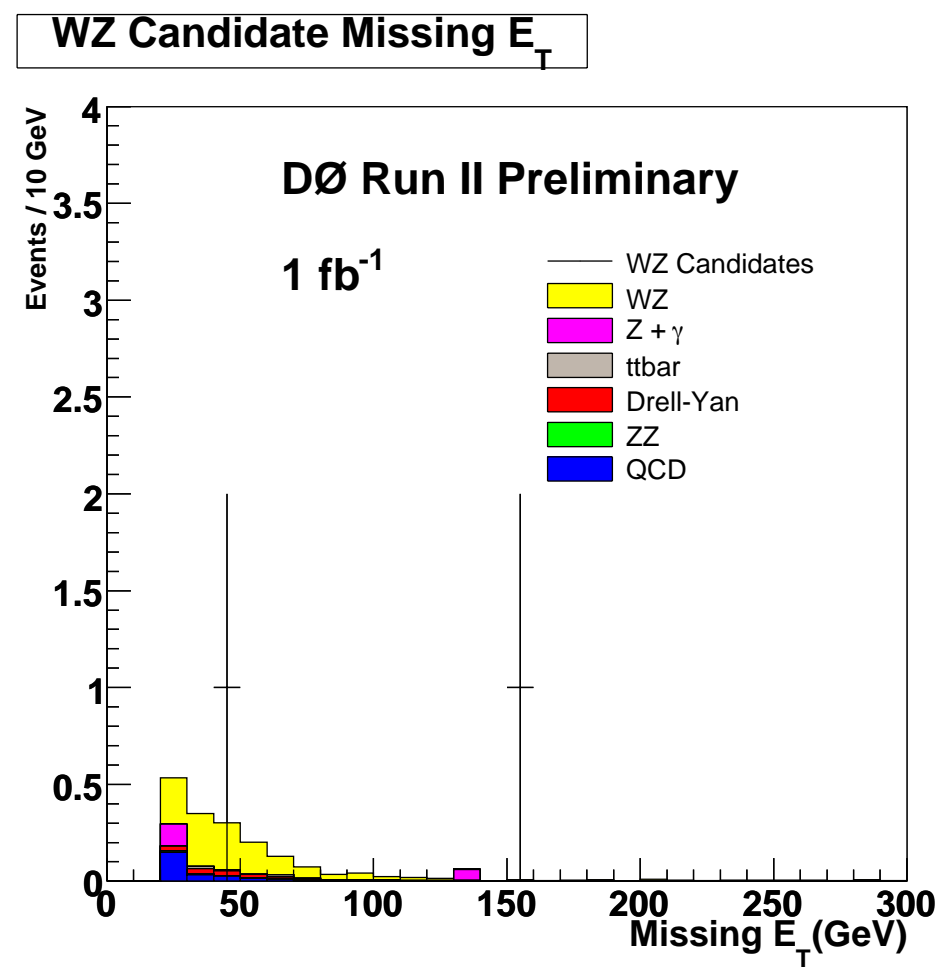

Figure B.2: The $\mathscr{E}_{T}$ distribution of the sample that did not pass the $M_{\ell \ell}$ cuts. 


\section{WZ Candidate Dilepton Mass}

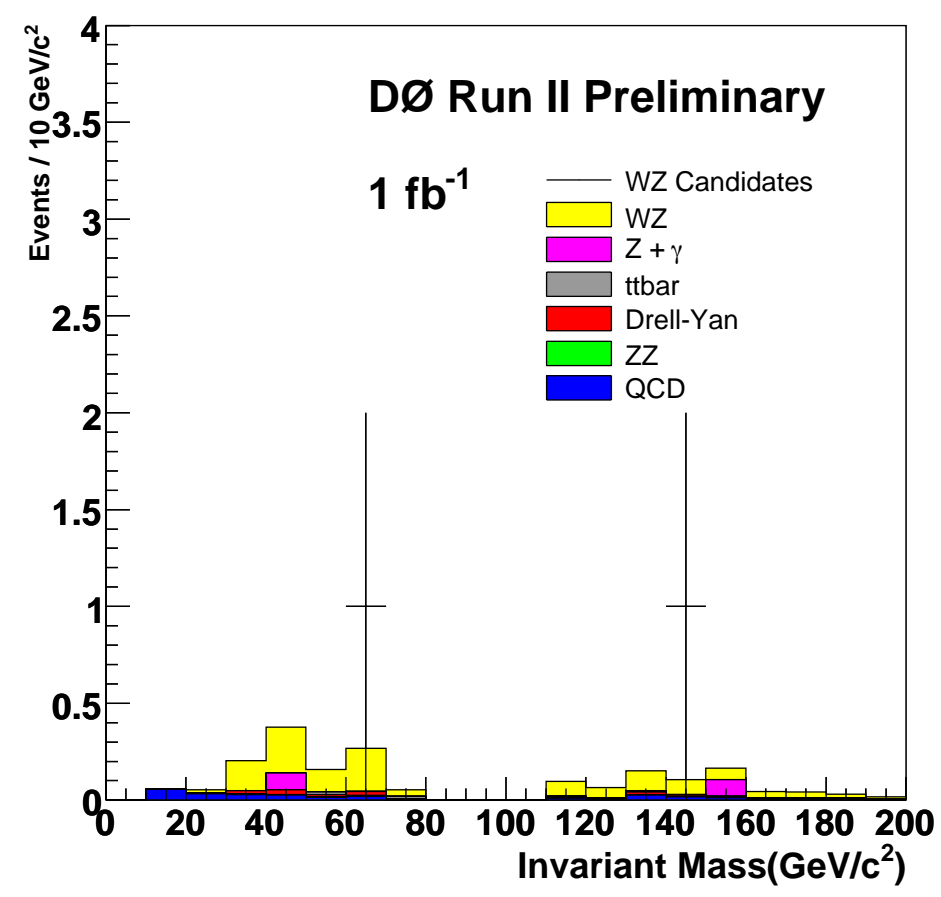

Figure B.3: The invariant mass distribution of the sample that did not pass the $M_{\ell \ell}$ cuts.

\section{WZ Candidate Dilepton Mass}

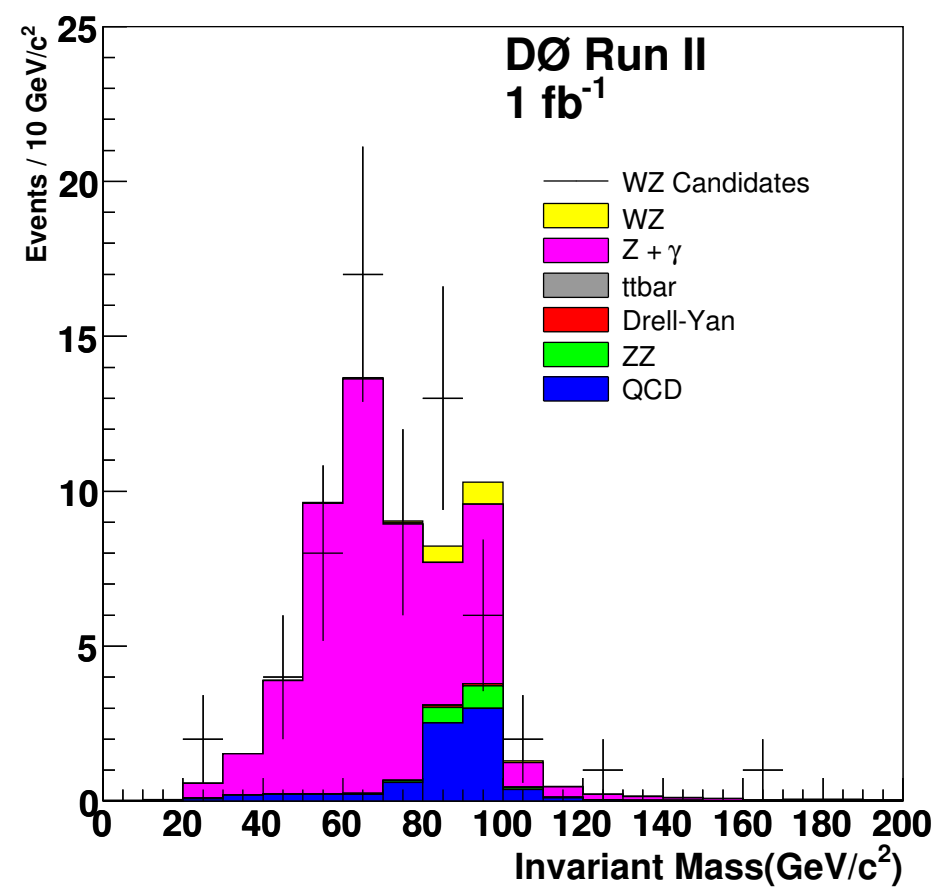

Figure B.4: The invariant mass distribution of the sample that did not pass the $\mathbb{E}_{T}$ cut. No invariant mass cut was applied in this sample. 


\section{APPENDIX C}

\section{Optimization}

Some variations on the selection criteria were studied in hopes that a more significant result would be possible. These include selection on $M_{T}$ so as to improve the correct $W$ daughters, variation of the $\mathbb{E}_{T}$ selection criteria from $15 \mathrm{GeV}$ to $25 \mathrm{GeV}$, and tighter cuts on the dilepton invariant mass around the $Z$ pole. The first optimization studied is to cut on the transverse mass $\left(M_{T}\right)$ of the $W$ boson leptons from the signal sample. The change in significance of the probability for the background to fluctuate to the expected signal plus background was studied as the $M_{T}$ cut was raised. The results of this study are summarized in table C.1.

Table C.1: This table shows the expected significance after applying a $M_{T}$ cut is applied to the data samples. The uncertainty on the background in this study is scaled with the background events. The significance is determined from the probability which the background would fluctuate to background plus expected signal as prescribed by $\mathrm{D} \emptyset$.

\begin{tabular}{|c|c|c|c|c|}
\hline Cut $(\mathrm{GeV})$ & Background (events) & MC Signal (events) & significance & $\frac{s}{\sqrt{s+b}}$ \\
\hline 0 & 4.47 & 9.2 & 3.0 & 2.5 \\
10 & 3.81 & 8.8 & 3.1 & 2.5 \\
20 & 3.36 & 8.4 & 3.1 & 2.4 \\
30 & 2.92 & 7.9 & 3.0 & 2.3 \\
40 & 2.31 & 7.3 & 3.1 & 2.4 \\
50 & 1.78 & 6.4 & 3.2 & 2.2 \\
\hline
\end{tabular}

A study taking into account all of the backgrounds and signal was performed on the $\mathbb{E}_{T}$ distribution shown in Figure C.1. The results of which are presented in table C.2. 
The study's goal was to maximize the significance of the selection criteria. The is done by calculating the significance of the probability for the background to fluctuate to the expected signal plus background as the $\mathbb{E}_{T}$ cut is raised in $5 \mathrm{GeV}$ increments.

\section{WZ Candidate Missing $\mathrm{E}_{\mathrm{T}}$}

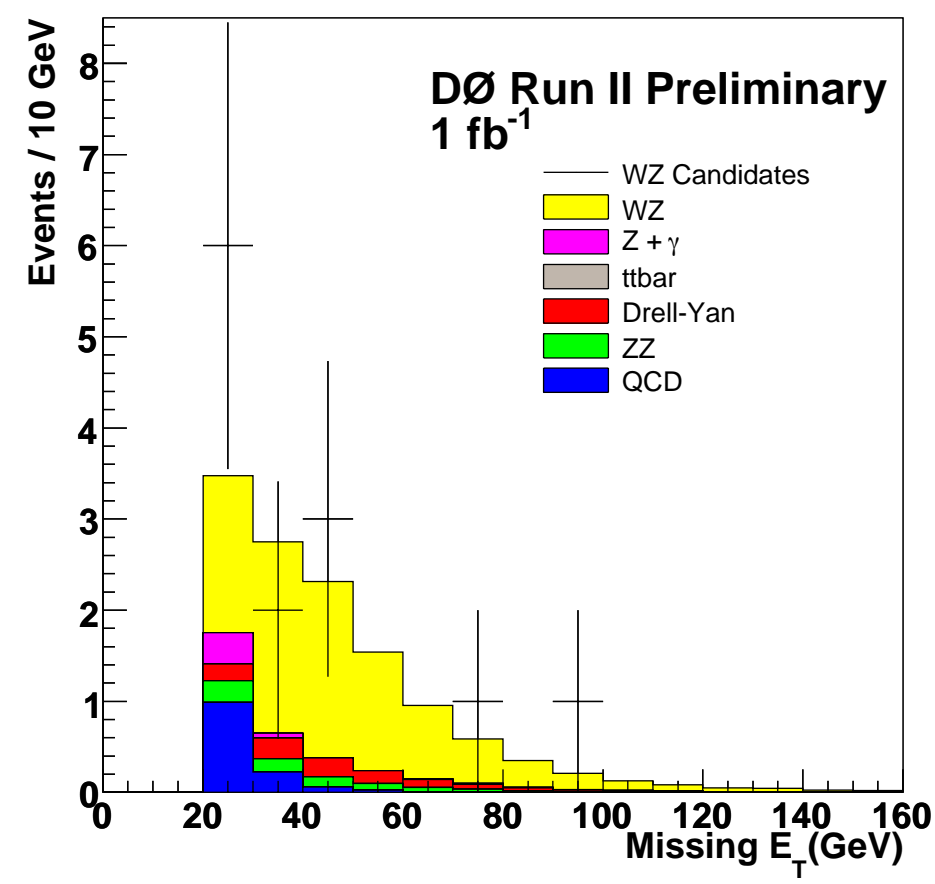

Figure C.1: Shown is the $\mathbb{E}_{T}$ of the candidate events along with the sum of the background estimates and expected signal. The MC are normalized to the data luminosity.

Table C.2: This table shows the expected significance if the $\mathbb{E}_{T}$ cut is increased in 5 GeV increments. The uncertainty on the background in this study is kept at 0.57 events. The significance is determined from the probability which the background would fluctuate to background plus expected signal as prescribed by $\mathrm{D} \varnothing$.

\begin{tabular}{|c|c|c|c|c|}
\hline Cut $(\mathrm{GeV})$ & Background (events) & MC Signal (events) & significance & $\frac{s}{\sqrt{s+b}}$ \\
\hline 20 & 4.47 & 9.2 & 3.0 & 2.49 \\
25 & 2.91 & 8.4 & 3.3 & 2.50 \\
30 & 2.11 & 7.5 & 3.1 & 2.41 \\
35 & 1.59 & 6.4 & 2.7 & 2.27 \\
40 & 1.27 & 5.4 & 2.5 & 2.08 \\
\hline
\end{tabular}

Finally the effect of tightening the dilepton invariant mass cut on the significance was studied. The results of this study are presented in Table C.3. 
Table C.3: This table shows the expected significance if the dilepton invariant mass cut is tightened. The uncertainty on the background in this study is scaled with the background. The significance is determined from the probability which the background would fluctuate to background plus expected signal as prescribed by $\mathrm{D} \varnothing$.

\begin{tabular}{|c|c|c|c|c|}
\hline Cut $(\mathrm{GeV})$ & Background (events) & MC Signal (events) & significance & $\frac{s}{\sqrt{s+b}}$ \\
\hline $50-130$ & 4.47 & 9.2 & 3.0 & 2.5 \\
$60-130$ & 4.33 & 9.1 & 3.1 & 2.5 \\
$70-130$ & 4.15 & 9.0 & 3.2 & 2.5 \\
$50-120$ & 4.37 & 9.1 & 3.1 & 2.5 \\
$60-120$ & 4.24 & 9.1 & 3.2 & 2.5 \\
$50-110$ & 4.18 & 9.0 & 3.2 & 2.5 \\
$70-120$ & 4.06 & 9.0 & 3.3 & 2.5 \\
$60-110$ & 4.05 & 9.0 & 3.3 & 2.5 \\
$70-110$ & 3.87 & 8.9 & 3.1 & 2.5 \\
\hline
\end{tabular}




\section{APPENDIX D}

\section{Trigger List}

The trigger list is chosen to maximize the efficiency of the signal. For the decay channels with $Z \rightarrow e e$ the single and di-EM triggers are used. For the decay channels with $Z \rightarrow \mu \mu$ the single muon triggers are used. When the single EM triggers are OR'ed

with the Di-EM triggers then the efficiency becomes essentially $100 \%$ for our signal [61]. Triggers were OR'd such that there was at least one unprescaled trigger for each run.

- v8-v10.3

- Single EM Triggers: EM_MX EM_HI EM_MX_SH EM_HI_SH EM_HI_2EM5_SH EM_MX_EMFR8 EM_HI_EMFR8

- Di-EM Triggers: 2EM_2MD7 2EM_2MD12 2EM_MD12_CEM10

- Single Muon Triggers: MU_W_L2M5_TRK10

- v10.3-v11

- Single EM Triggers: EM_MX EM_HI EM_MX_SH EM_HI_SH EM_HI_2EM5_SH EM_MX_EMFR8 EM_HI_EMFR8

- Di-EM Triggers: 2EM_2MD7 2EM_2MD12 2EM_MD12_CEM10

- Single Muon Triggers: MUW_A_L2M3_TRK10

- $\mathrm{v} 12$ 
- Single EM Triggers: E1_SHT20 E2_SHT20 E3_SHT20 E1_SH30 E2_SH30 E3_SH30 E1_L50 E1_VL70

- Di-EM Triggers: E1_2SH8 E2_2SH8 E3_2SH8 E1_2L15_SH15 E2_2L15_SH15 E3_2L15_SH15

- Single Muon Triggers: MUW_W_L2M3_TRK10

- v13

- Single EM Triggers: E1_SHT20 E2_SHT20 E3_SHT20 E4_SHT20 E1_SH30 E2_SH30 E3_SH30 E4_SH30 E1_L50 E1_NC90

- Di-EM Triggers: E1_2L20 E2_2L20 E3_2L20 E4_2L20 E20_2L20 E21_2L20 E22_2L20 E23_2L20 E25_2L20 E1_2L15_SH15 E2_2L15_SH15 E3_2L15_SH15 E4_2L15_SH15 E20_2L15_SH15 E21_2L15_SH15 E22_2L15_SH15 E23_2L15_SH15 E25_2L15_SH15 E1_2SH8 E2_2SH8 E3_2SH8 E4_2SH8 E20_2SH8 E21_2SH8 E22_2SH8 E23_2SH8 E25_2SH8

- Single Muon Triggers: MUH1_TK12

- $\mathrm{v} 13.2$

- Single EM Triggers: E1_SHT22 E2_SHT22 E3_SHT22 E4_SHT22 E1_SH30 E2_SH30 E3_SH30 E4_SH30 E1_L70 E1_NC90

- Di-EM Triggers: E1_2L20 E2_2L20 E3_2L20 E4_2L20 E20_2L20 E21_2L20 E22_2L20 E23_2L20 E25_2L20 E1_2L15_SH15 E2_2L15_SH15 E3_2L15_SH15 E4_2L15_SH15 E20_2L15_SH15 E21_2L15_SH15 E22_2L15_SH15 E23_2L15_SH15 E25_2L15_SH15 E1_2SH8 E2_2SH8 E3_2SH8 E4_2SH8 E20_2SH8 E21_2SH8 E22_2SH8 E23_2SH8 E25_2SH8

- Single Muon Triggers: MUH1_TK12

- v14 
- Single EM Triggers: E1_SHT25 E3_SHT25 E4_SHT25 E1_SH35 E3_SH35 E4_SH35 E1_L70

- Di-EM Triggers: E1_2L20_L25 E2_2L20_L25 E3_2L20_L25 E4_2L20_L25 E5_2L20_L25 E6_2L20_L25 E1_2L15_SH15_L20 E2_2L15_SH15_L20 E3_2L15_SH15_L20 E4_2L15_SH15_L20 E5_2L15_SH15_L20 E6_2L15_SH15_L20 E1_2SH10_SH15 E2_2SH10_SH15 E3_2SH10_SH15 E4_2SH10_SH15 E5_2SH10_SH15 E6_2SH10_SH15

- Single Muon Triggers: MUH1_TK12_TLM12

- v14.60

- Single EM Triggers: E1_SHT25 E3_SHT25 E4_SHT25 E1_SH35 E3_SH35 E4_SH35 E1_L70

- Di-EM Triggers: E1_2L20_L25 E2_2L20_L25 E3_2L20_L25 E4_2L20_L25 E5_2L20_L25 E6_2L20_L25 E1_2L15_SH15_L20 E2_2L15_SH15_L20 E3_2L15_SH15_L20 E4_2L15_SH15_L20 E5_2L15_SH15_L20 E6_2L15_SH15_L20 E1_2SH10_SH15 E2_2SH10_SH15 E3_2SH10_SH15 E4_2SH10_SH15 E5_2SH10_SH15 E6_2SH10_SH15

- Single Muon Triggers: MUH1_TK12_TLM12 


\section{APPENDIX E}

\section{Candidate List}

Table E.1 shows the list of candidates found in the data set listed in Tables 6.1, 6.2 and 6.3. The table lists the event leptons and their kinematic properties. Table E.2 lists the same events with their vector boson physical and kinematic properties. Following the tables are the event views of the 13 candidates.

Table E.1: Presented are the kinematic properties of each lepton for all of the candidate events.

\begin{tabular}{|c|c|c|c|c|c|c|c|c|c|c|c|c|}
\hline $\begin{array}{c}\text { Run } \\
\text { Number }\end{array}$ & $\begin{array}{c}\text { Event } \\
\text { Number }\end{array}$ & $\begin{array}{l}\mathscr{E}_{T} \\
\mathrm{GeV}\end{array}$ & $\begin{array}{l}\phi_{E_{T}} \\
\mathrm{rad} .\end{array}$ & $\begin{array}{l}\text { lepton } \\
\text { family }\end{array}$ & $\begin{array}{c}\text { charge } \\
\left|q_{e}\right|\end{array}$ & $\begin{array}{c}\text { Energy } \\
\text { GeV }\end{array}$ & $\begin{array}{c}p_{T} \\
\mathrm{GeV} / \mathrm{c}\end{array}$ & $\begin{array}{c}\phi \\
\mathrm{rad} .\end{array}$ & $\eta$ & $\begin{array}{c}p_{x} \\
\mathrm{GeV} / \mathrm{c}\end{array}$ & $\begin{array}{c}p_{y} \\
\mathrm{GeV} / \mathrm{c}\end{array}$ & $\begin{array}{c}p z \\
\mathrm{GeV} / \mathrm{c}\end{array}$ \\
\hline 188371 & 23177216 & 20.50 & 0.25 & $\begin{array}{l}e \\
\mu \\
\mu\end{array}$ & $\begin{array}{l}+1 \\
+1 \\
-1\end{array}$ & $\begin{array}{l}89.90 \\
96.02 \\
42.20\end{array}$ & $\begin{array}{l}34.57 \\
56.44 \\
36.68\end{array}$ & $\begin{array}{l}1.90 \\
3.65 \\
6.12\end{array}$ & $\begin{array}{l}1.61 \\
0.66 \\
0.54\end{array}$ & $\begin{array}{c}-11.15 \\
-49.34 \\
36.21\end{array}$ & $\begin{array}{c}32.73 \\
-27.40 \\
-5.82\end{array}$ & $\begin{array}{l}82.99 \\
39.71 \\
20.86\end{array}$ \\
\hline 191882 & 21112860 & 22.75 & 3.59 & $\begin{array}{l}e \\
e \\
e\end{array}$ & $\begin{array}{l}+1 \\
-1 \\
-1\end{array}$ & $\begin{array}{c}105.91 \\
55.59 \\
23.81\end{array}$ & $\begin{array}{l}73.1 \\
34.94 \\
15.62\end{array}$ & $\begin{array}{l}0.26 \\
3.38 \\
2.12\end{array}$ & $\begin{array}{c}-0.92 \\
-1.04 \\
0.98\end{array}$ & $\begin{array}{c}70.73 \\
-33.99 \\
-8.09\end{array}$ & $\begin{array}{l}18.46 \\
-8.09 \\
13.36\end{array}$ & $\begin{array}{c}-76.63 \\
-43.23 \\
17.97\end{array}$ \\
\hline 192868 & 66474158 & 37.19 & 1.31 & $\begin{array}{l}e \\
\mu \\
\mu\end{array}$ & $\begin{array}{l}-1 \\
+1 \\
-1\end{array}$ & $\begin{array}{c}29.86 \\
131.32 \\
40.24\end{array}$ & $\begin{array}{l}23.52 \\
87.09 \\
38.65\end{array}$ & $\begin{array}{l}1.31 \\
4.00 \\
6.12\end{array}$ & $\begin{array}{c}0.72 \\
-0.97 \\
-0.31\end{array}$ & $\begin{array}{c}6.03 \\
-51.17 \\
38.16\end{array}$ & $\begin{array}{c}22.74 \\
-65.70 \\
-6.16\end{array}$ & $\begin{array}{c}18.38 \\
-98.28 \\
-12.11\end{array}$ \\
\hline 194259 & 5929362 & 32.76 & 3.35 & $\begin{array}{l}e \\
e \\
e\end{array}$ & $\begin{array}{l}+1 \\
+1 \\
-1\end{array}$ & $\begin{array}{c}58.29 \\
280.48 \\
115.78\end{array}$ & $\begin{array}{l}55.89 \\
51.04 \\
39.23\end{array}$ & $\begin{array}{l}5.67 \\
3.64 \\
1.23\end{array}$ & $\begin{array}{c}-0.29 \\
2.39 \\
1.75\end{array}$ & $\begin{array}{c}45.56 \\
-44.79 \\
13.08\end{array}$ & $\begin{array}{c}-32.38 \\
-24.49 \\
36.98\end{array}$ & $\begin{array}{l}-16.57 \\
275.79 \\
108.93\end{array}$ \\
\hline 195167 & 18587081 & 44.09 & 4.43 & $\begin{array}{l}e \\
e \\
e \\
\mu\end{array}$ & $\begin{array}{l}+1 \\
-1 \\
-1\end{array}$ & $\begin{array}{c}39.03 \\
163.75 \\
47.77\end{array}$ & $\begin{array}{c}33.46 \\
29.5 \\
26.23\end{array}$ & $\begin{array}{l}1.71 \\
4.91 \\
1.25\end{array}$ & $\begin{array}{c}-0.57 \\
-2.4 \\
1.21\end{array}$ & $\begin{array}{c}-4.51 \\
5.87 \\
8.18\end{array}$ & $\begin{array}{c}33.15 \\
-28.91 \\
24.92\end{array}$ & $\begin{array}{c}-20.1 \\
161.07 \\
39.93\end{array}$ \\
\hline 204318 & 69485771 & 23.68 & 2.39 & $\begin{array}{l}e \\
\mu \\
\mu\end{array}$ & $\begin{array}{l}-1 \\
-1 \\
+1\end{array}$ & $\begin{array}{c}102.9 \\
159.74 \\
112.69\end{array}$ & $\begin{array}{c}99.99 \\
85.2 \\
77.69\end{array}$ & $\begin{array}{l}1.83 \\
5.83 \\
4.42\end{array}$ & $\begin{array}{l}0.24 \\
1.24 \\
0.92\end{array}$ & $\begin{array}{c}-25.34 \\
76.69 \\
-22.3\end{array}$ & $\begin{array}{c}96.72 \\
-37.11 \\
-74.42\end{array}$ & $\begin{array}{c}24.31 \\
-66.36 \\
40.58\end{array}$ \\
\hline 204938 & 6268052 & 45.49 & 5.17 & $\begin{array}{l}\mu \\
\mu \\
\mu\end{array}$ & $\begin{array}{l}+1 \\
+1 \\
-1\end{array}$ & $\begin{array}{c}104.48 \\
80.34 \\
52.65\end{array}$ & $\begin{array}{c}52.1 \\
45.27 \\
33.54\end{array}$ & $\begin{array}{l}2.99 \\
2.15 \\
5.82\end{array}$ & $\begin{array}{c}1.32 \\
-1.18 \\
1.02\end{array}$ & $\begin{array}{c}-51.51 \\
-24.78 \\
30.03\end{array}$ & $\begin{array}{c}7.84 \\
37.89 \\
-14.94\end{array}$ & $\begin{array}{c}90.56 \\
-66.36 \\
40.58\end{array}$ \\
\hline 205114 & 3409480 & 45.57 & 4.62 & $\begin{array}{l}\mu \\
\mu \\
\mu\end{array}$ & $\begin{array}{l}-1 \\
+1 \\
-1\end{array}$ & $\begin{array}{c}18.14 \\
90.45 \\
45.1\end{array}$ & $\begin{array}{l}15.52 \\
37.00 \\
17.36\end{array}$ & $\begin{array}{l}1.55 \\
1.77 \\
5.99\end{array}$ & $\begin{array}{c}1.02 \\
-0.57 \\
-1.54 \\
1.61\end{array}$ & $\begin{array}{c}0.00 \\
0.28 \\
-7.37 \\
16.6\end{array}$ & $\begin{array}{c}-14.94 \\
15.52 \\
36.26 \\
-5.08\end{array}$ & $\begin{array}{c}40.00 \\
-9.4 \\
-82.53 \\
41.63\end{array}$ \\
\hline 206332 & 20605317 & 24.83 & 0.18 & $\begin{array}{l}\mu \\
\mu \\
\mu \\
\mu\end{array}$ & $\begin{array}{l}-1 \\
-1 \\
+1\end{array}$ & $\begin{array}{c}69.7 \\
31.00 \\
66.1\end{array}$ & $\begin{array}{l}30.86 \\
25.49 \\
49.13\end{array}$ & $\begin{array}{l}3.56 \\
5.91 \\
2.78\end{array}$ & $\begin{array}{l}1.01 \\
1.45 \\
0.65 \\
0.81\end{array}$ & $\begin{array}{c}-28.18 \\
23.74 \\
-46.03\end{array}$ & $\begin{array}{c}-12.59 \\
-9.3 \\
17.18\end{array}$ & $\begin{array}{c}62.5 \\
17.64 \\
44.21\end{array}$ \\
\hline 207094 & 10178395 & 78.77 & 5.56 & $\begin{array}{l}e \\
\mu \\
\mu\end{array}$ & $\begin{array}{l}-1 \\
+1 \\
-1\end{array}$ & $\begin{array}{l}105.87 \\
115.43 \\
36.35\end{array}$ & $\begin{array}{c}46.84 \\
113.46 \\
22.02\end{array}$ & $\begin{array}{l}0.28 \\
2.56 \\
4.33\end{array}$ & $\begin{array}{l}-1.46 \\
-0.19 \\
-1.09\end{array}$ & $\begin{array}{c}45.00 \\
-94.86 \\
-8.31\end{array}$ & $\begin{array}{c}13.00 \\
62.26 \\
-20.39\end{array}$ & $\begin{array}{l}-94.94 \\
-21.21 \\
-28.92\end{array}$ \\
\hline 207596 & 12955559 & 22.61 & 1.12 & $\begin{array}{l}e \\
\mu \\
\mu\end{array}$ & $\begin{array}{l}+1 \\
+1 \\
+1\end{array}$ & $\begin{array}{l}49.58 \\
41.71 \\
91.49\end{array}$ & $\begin{array}{l}30.78 \\
33.64 \\
46.62\end{array}$ & $\begin{array}{l}4.96 \\
2.01 \\
4.45\end{array}$ & $\begin{array}{l}1.06 \\
0.68 \\
1.29\end{array}$ & $\begin{array}{c}7.50 \\
-14.43 \\
-11.96\end{array}$ & $\begin{array}{c}-29.85 \\
30.39 \\
-45.06\end{array}$ & $\begin{array}{l}38.86 \\
24.67 \\
78.72\end{array}$ \\
\hline 207769 & 23761167 & 30.97 & 0.07 & $\begin{array}{l}\mu \\
\mu \\
\mu\end{array}$ & $\begin{array}{l}-1 \\
+1 \\
-1\end{array}$ & $\begin{array}{c}270.11 \\
80.36 \\
40.69\end{array}$ & $\begin{array}{l}62.34 \\
46.57 \\
36.63\end{array}$ & $\begin{array}{l}3.71 \\
0.72 \\
2.79\end{array}$ & $\begin{array}{c}2.15 \\
-1.14 \\
-0.47\end{array}$ & $\begin{array}{c}-52.42 \\
35.14 \\
-34.33\end{array}$ & $\begin{array}{c}10.33 .74 \\
30.56 \\
12.78\end{array}$ & $\begin{array}{l}262.82 \\
-65.49 \\
-17.73\end{array}$ \\
\hline 210156 & 24837747 & 39.10 & 2.69 & $\begin{array}{l}e \\
\mu \\
\mu\end{array}$ & $\begin{array}{l}+1 \\
-1 \\
+1\end{array}$ & $\begin{array}{c}18.04 \\
75.45 \\
102.36\end{array}$ & $\begin{array}{l}17.96 \\
68.89 \\
58.80\end{array}$ & $\begin{array}{l}1.95 \\
4.96 \\
0.11\end{array}$ & $\begin{array}{c}-0.09 \\
-0.433 \\
-1.15\end{array}$ & $\begin{array}{l}-6.67 \\
16.60 \\
58.44\end{array}$ & $\begin{array}{c}16.68 \\
-66.87 \\
6.54\end{array}$ & $\begin{array}{l}-1.65 \\
-30.78 \\
-83.78\end{array}$ \\
\hline
\end{tabular}


Table E.2: Presented are the vector boson properties for each candidate event. One event has zero transverse mass because the $\phi$ of the $\mathscr{E}_{T}$ and the $e$ are the same, with in resolution effects, and therefore reconstruct a zero $M_{T}$.

\begin{tabular}{|c|c|c|c|c|c|c|}
\hline $\begin{array}{c}\text { Run } \\
\text { Number }\end{array}$ & $\begin{array}{c}\text { Event } \\
\text { Number }\end{array}$ & $\begin{array}{c}\text { Invariant } \\
\text { Mass GeV/c }\end{array}$ & $\begin{array}{c}\text { Transverse } \\
\text { Mass GeV/c }\end{array}$ & $\begin{array}{c}Z_{p_{T}} \\
\mathrm{GeV} / \mathrm{c}\end{array}$ & $\eta_{Z}$ & $\begin{array}{c}\phi_{Z} \\
\mathrm{rad} .\end{array}$ \\
\hline \hline 195167 & 18587081 & 90.99 & 68.0 & 4.46 & -1.44 & 1.26 \\
204938 & 6268052 & 82.6 & 90.53 & 21.51 & 1.20 & 3.49 \\
206332 & 20605317 & 70.91 & 54.97 & 23.71 & 0.75 & 2.80 \\
207596 & 12955559 & 78.60 & 49.58 & 30.42 & 1.04 & 3.66 \\
205114 & 3409480 & 124.5 & 35.59 & 32.45 & -0.31 & 1.29 \\
194259 & 5929362 & 88.58 & 80.54 & 34.08 & 2.11 & 2.77 \\
188371 & 23177216 & 86.07 & 63.07 & 35.65 & 0.61 & 4.33 \\
207769 & 2376116 & 76.54 & 85.16 & 43.40 & -0.84 & 1.55 \\
191882 & 21112860 & 91.91 & 5.91 & 70.26 & -0.49 & 0.47 \\
192868 & 66474158 & 108.34 & 0.00 & 74.36 & -0.76 & 4.45 \\
210156 & 24837747 & 95.97 & 51.54 & 96.18 & -0.77 & 5.61 \\
207094 & 10178395 & 90.32 & 58.41 & 111.35 & -0.34 & 2.76 \\
204318 & 69485771 & 108.71 & 26.89 & 124.0 & 1.09 & 5.17 \\
\hline
\end{tabular}




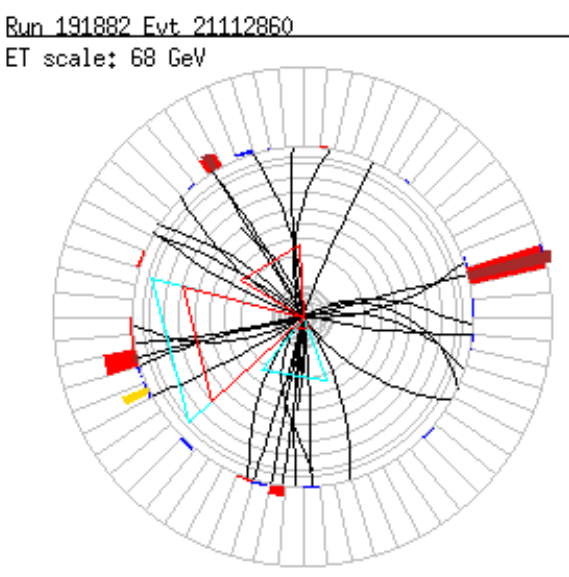

(a) $r-\phi$ view
Run 191882 Eut 21112860

E scale: $65 \mathrm{GeV}$

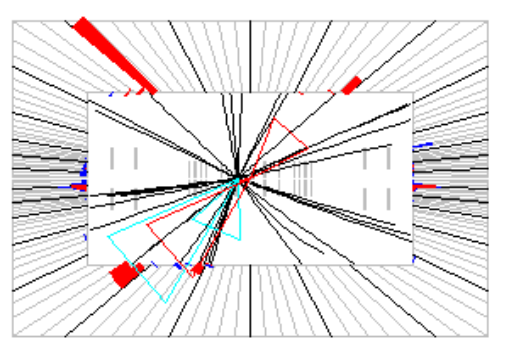

Run 191882 Evt 21112860

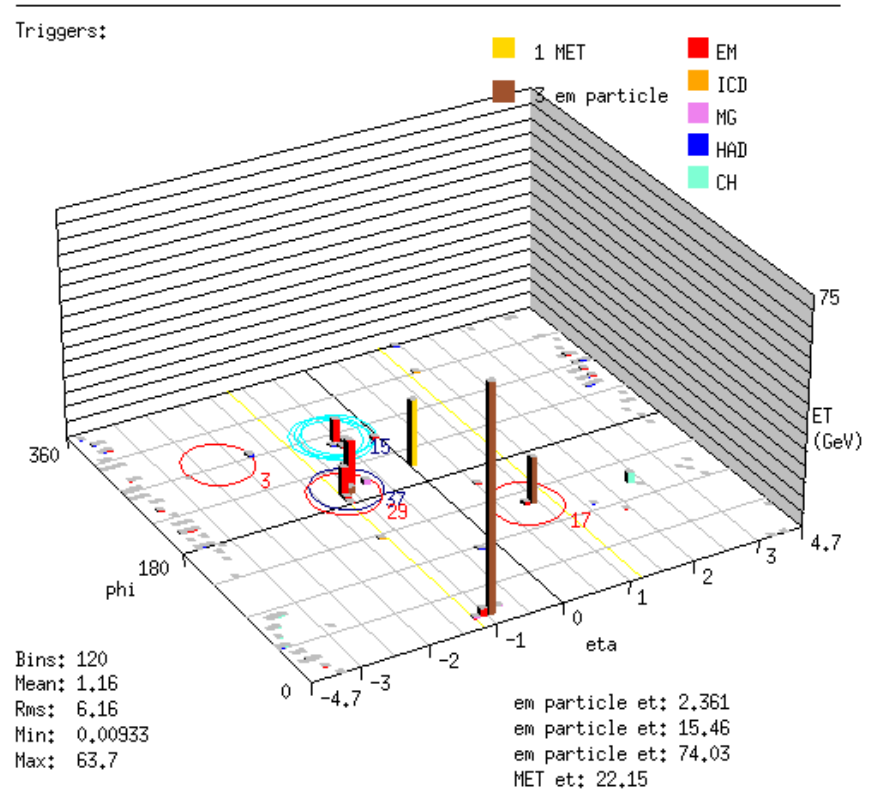

(c) Lego Plot

Figure E.1: Event View: Run Number - 191882 Event Number - 21112860 


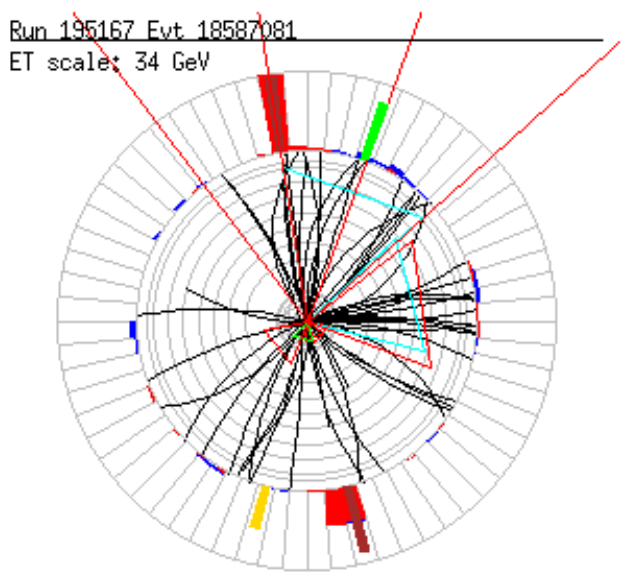

(a) $r-\phi$ view

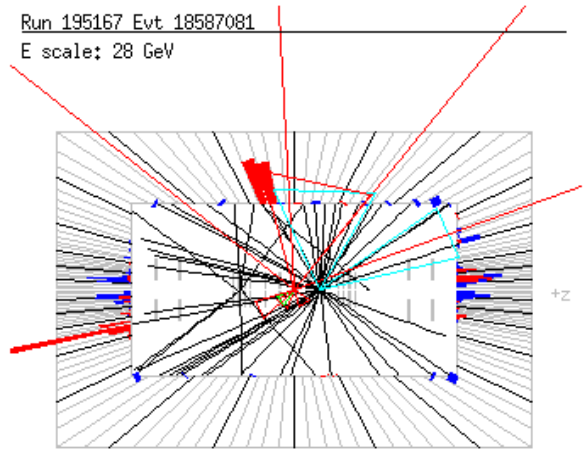

(b) $r-z$ view

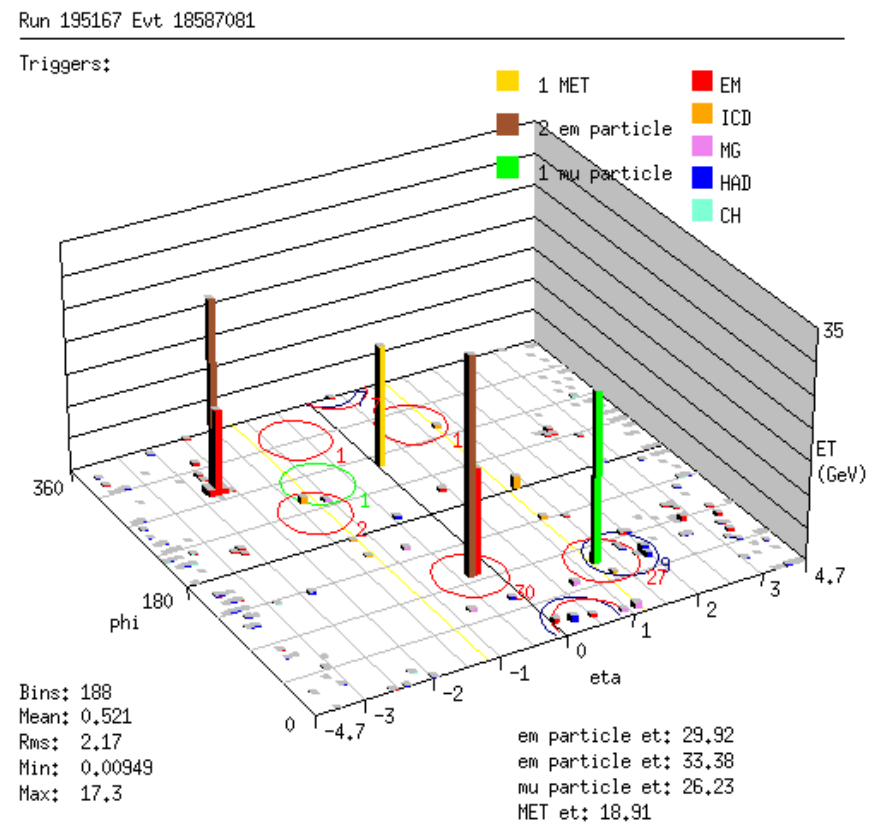

(c) Lego Plot

Figure E.2: Event View: Run Number - 195167 Event Number - 18587081 


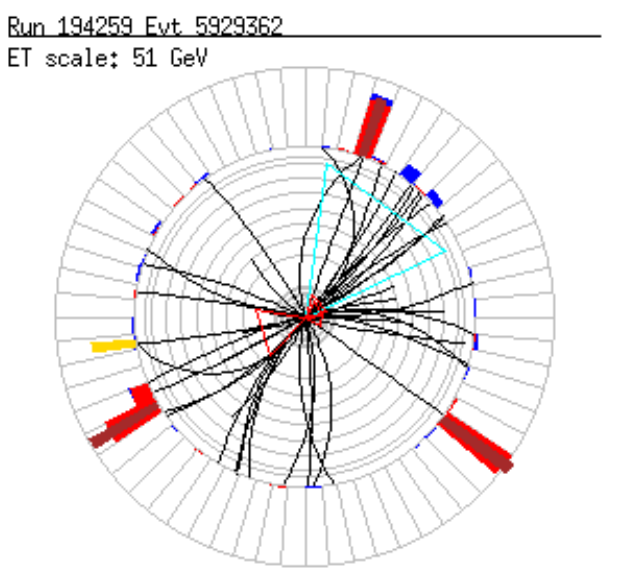

(a) $r-\phi$ view

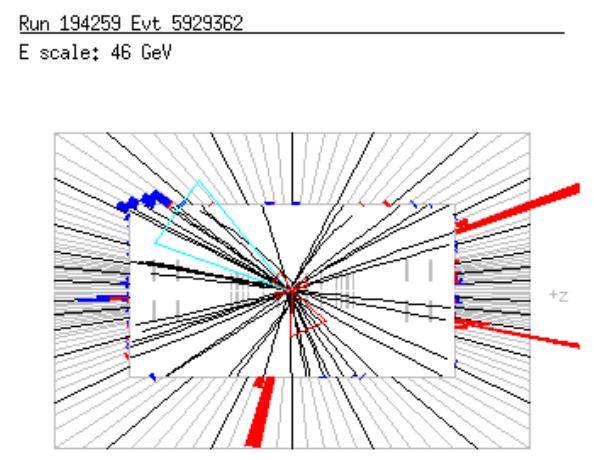

(b) $r-z$ view

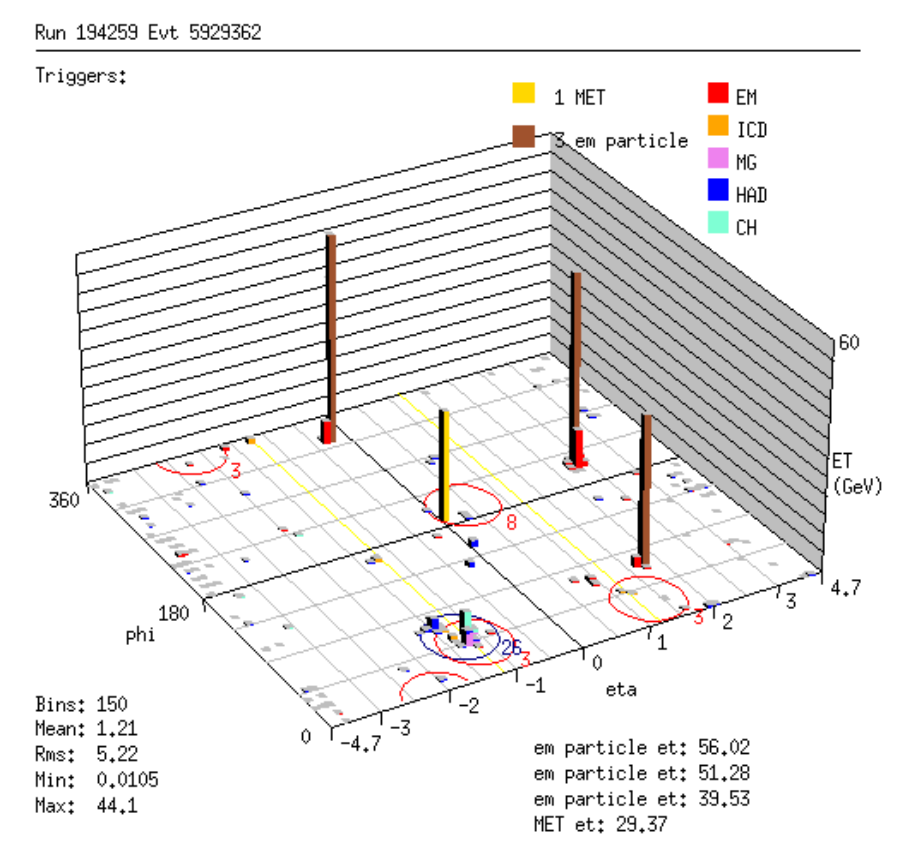

(c) Lego Plot

Figure E.3: Event View: Run Number - 194259 Event Number - 5929362 


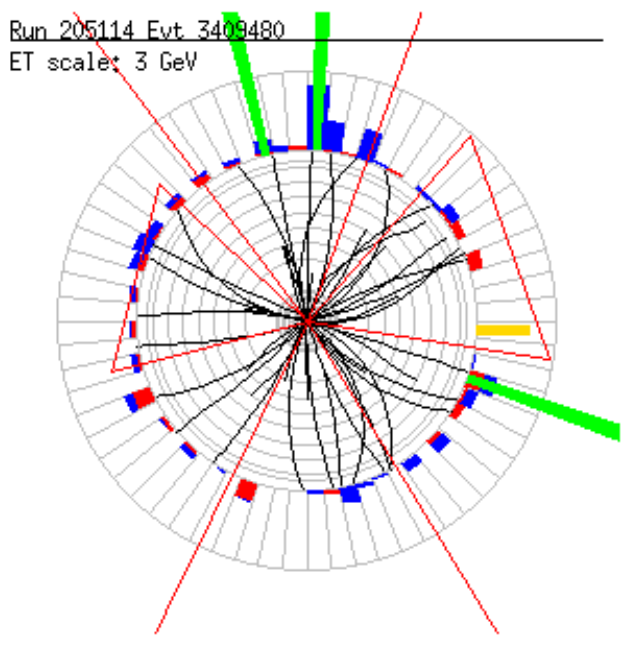

(a) $r-\phi$ view

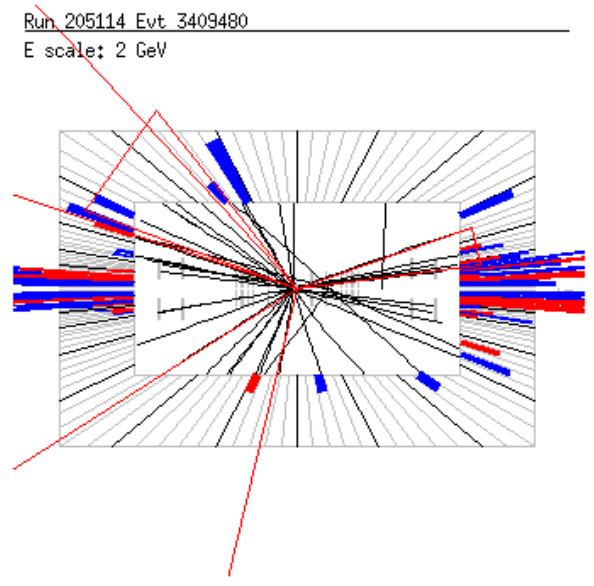

(b) $r-z$ view

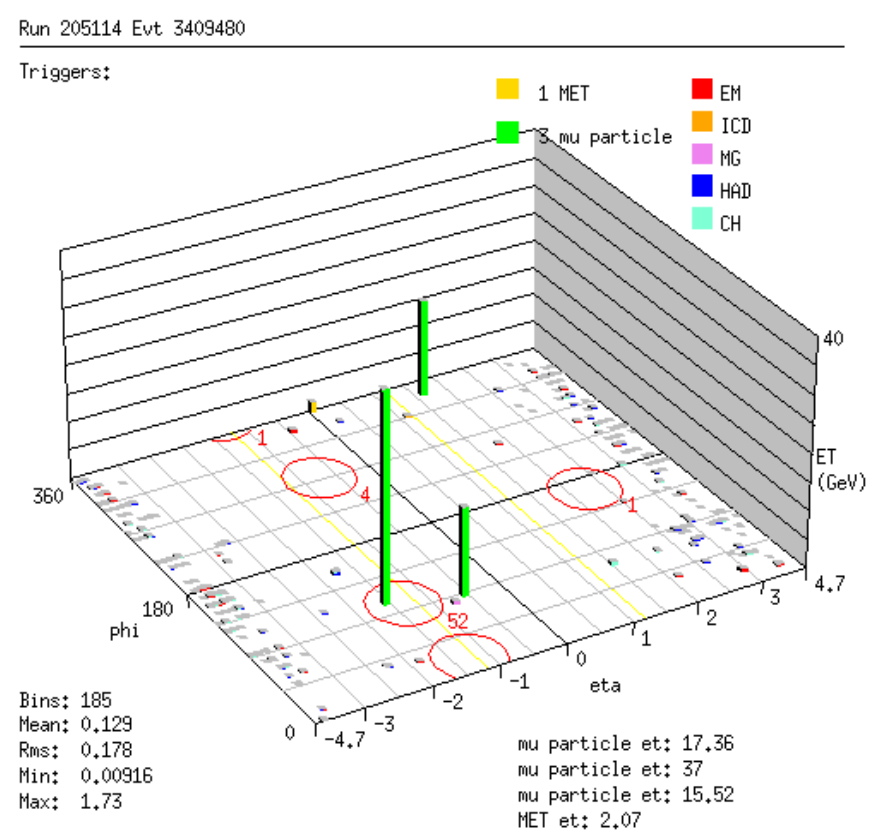

(c) Lego Plot

Figure E.4: Event View: Run Number - 205114 Event Number - 3409480 


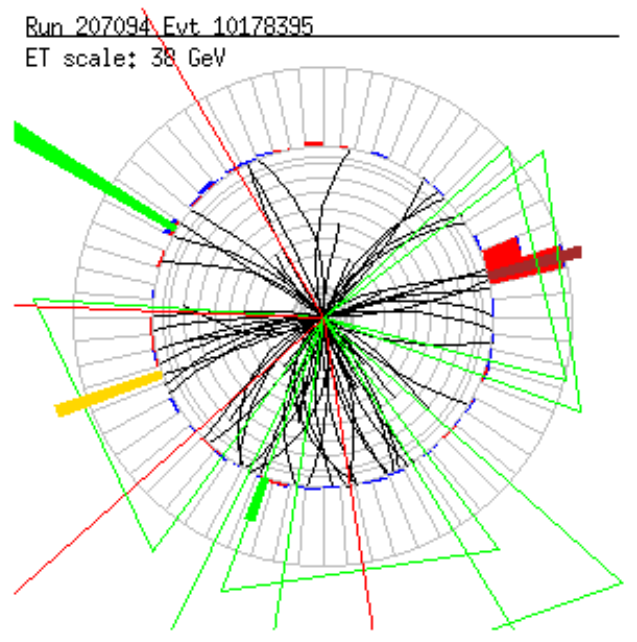

(a) $r-\phi$ view

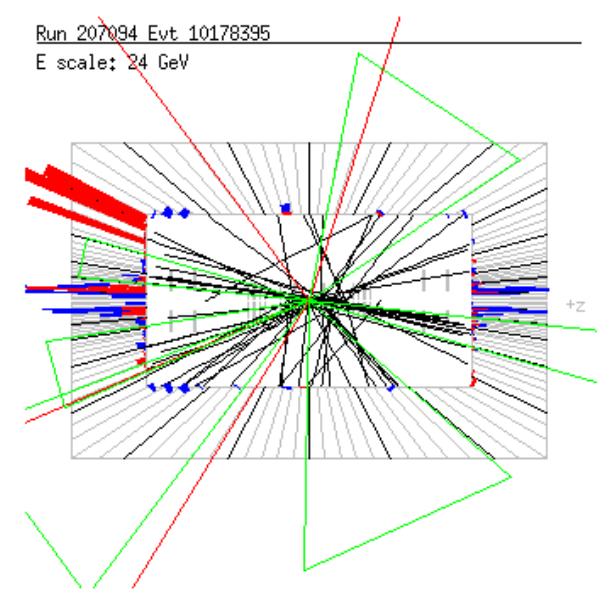

(b) $r-z$ view

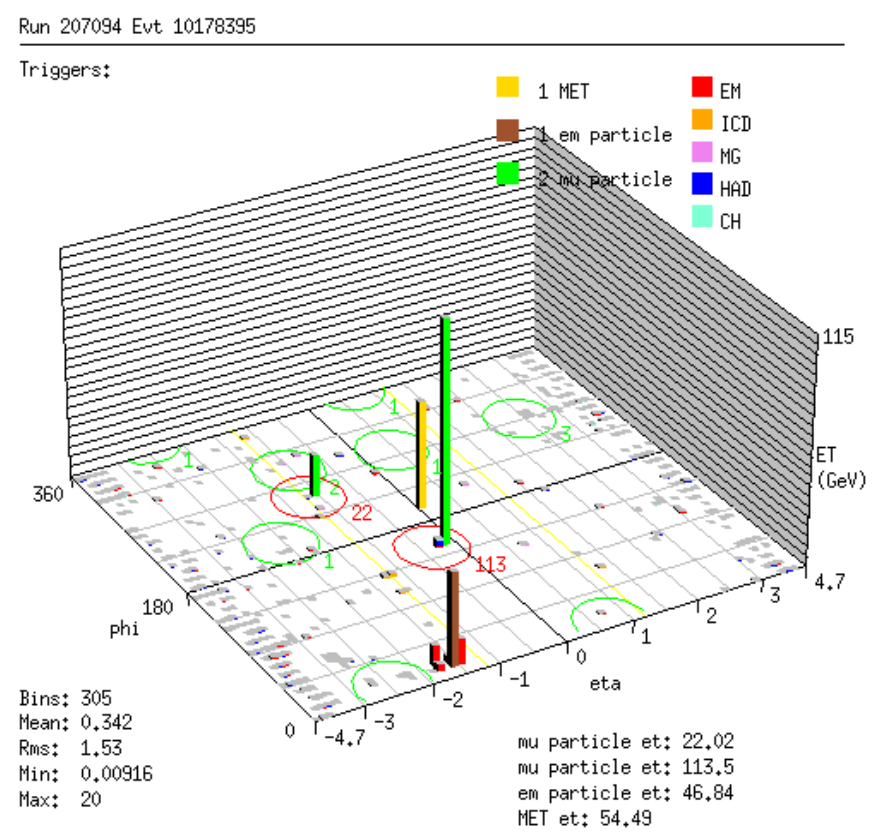

(c) Lego Plot

Figure E.5: Event View: Run Number - 207094 Event Number - 10178395 


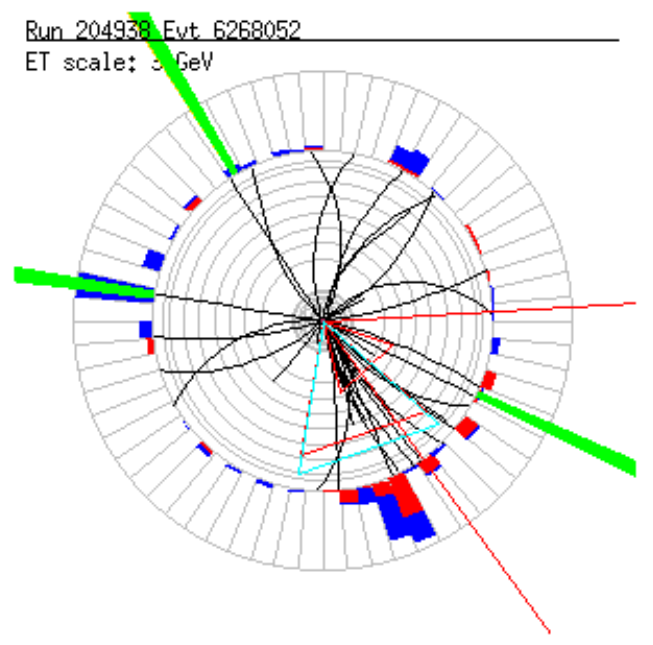

(a) $r-\phi$ view

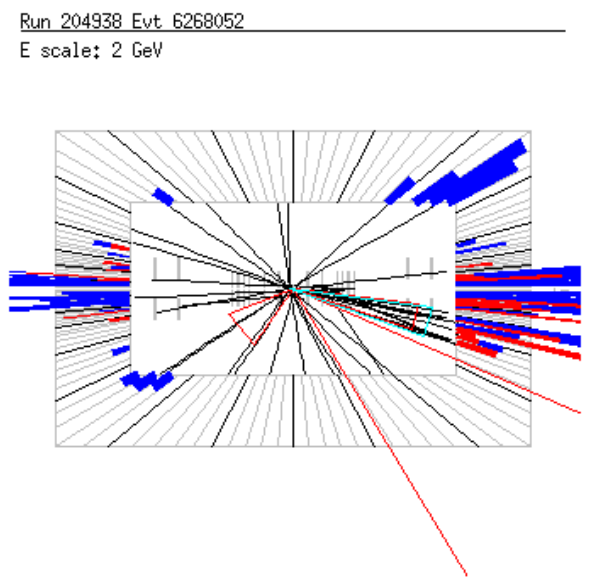

(b) $r-z$ view

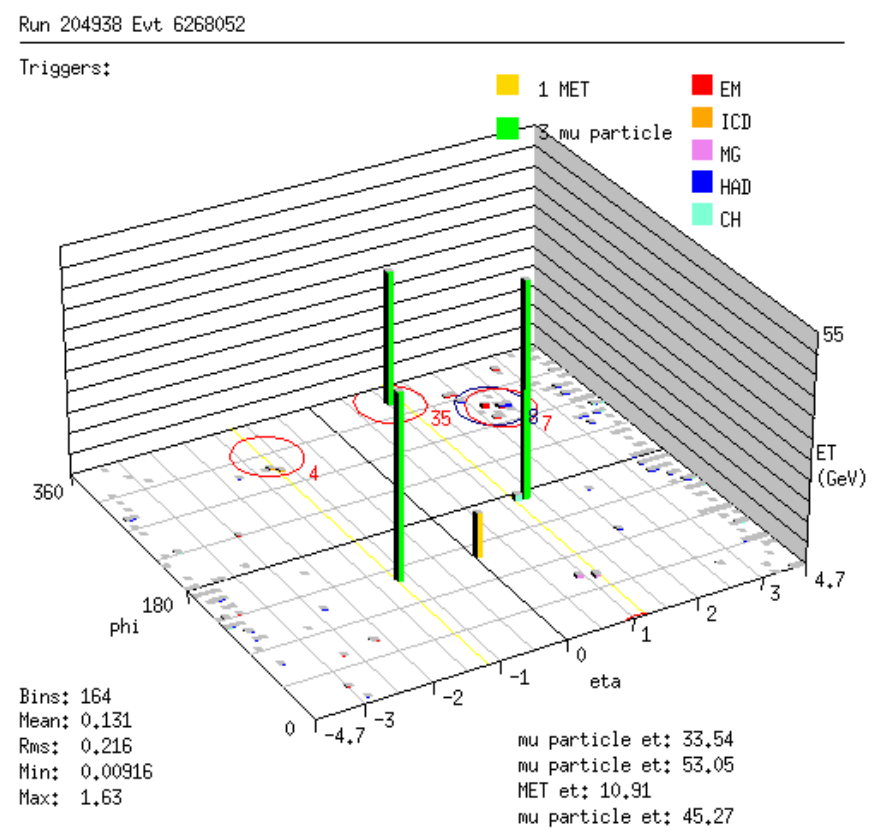

(c) Lego Plot

Figure E.6: Event View: Run Number - 204938 Event Number - 6268052 


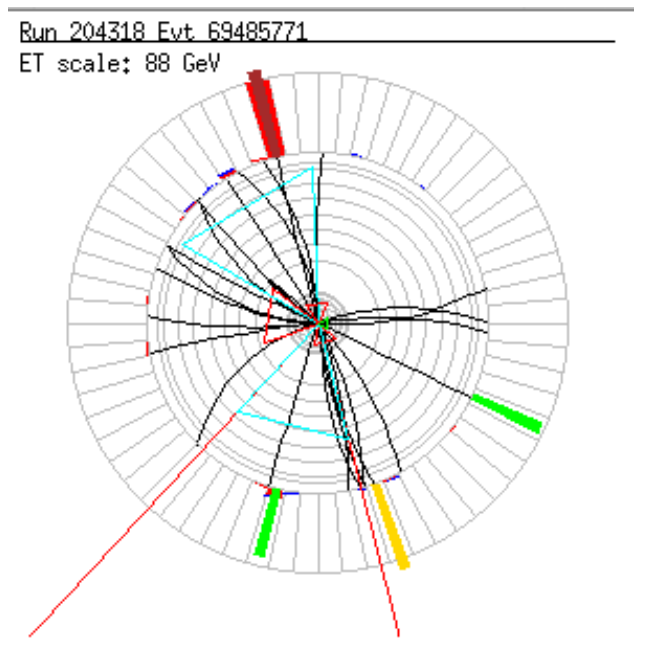

(a) $r-\phi$ view

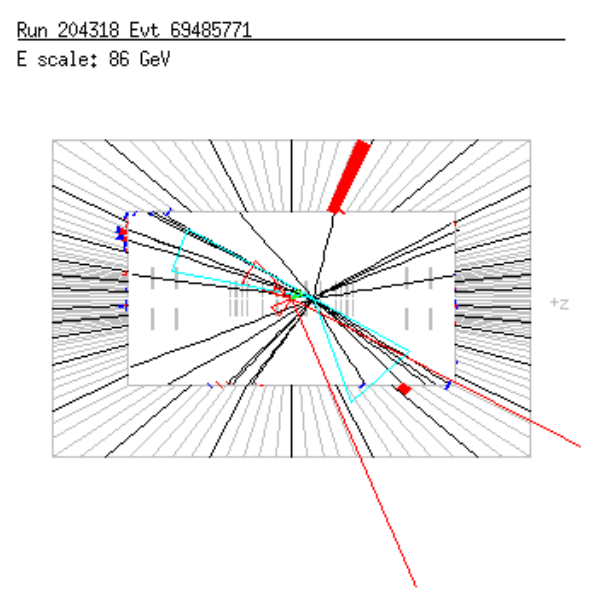

(b) $r-z$ view

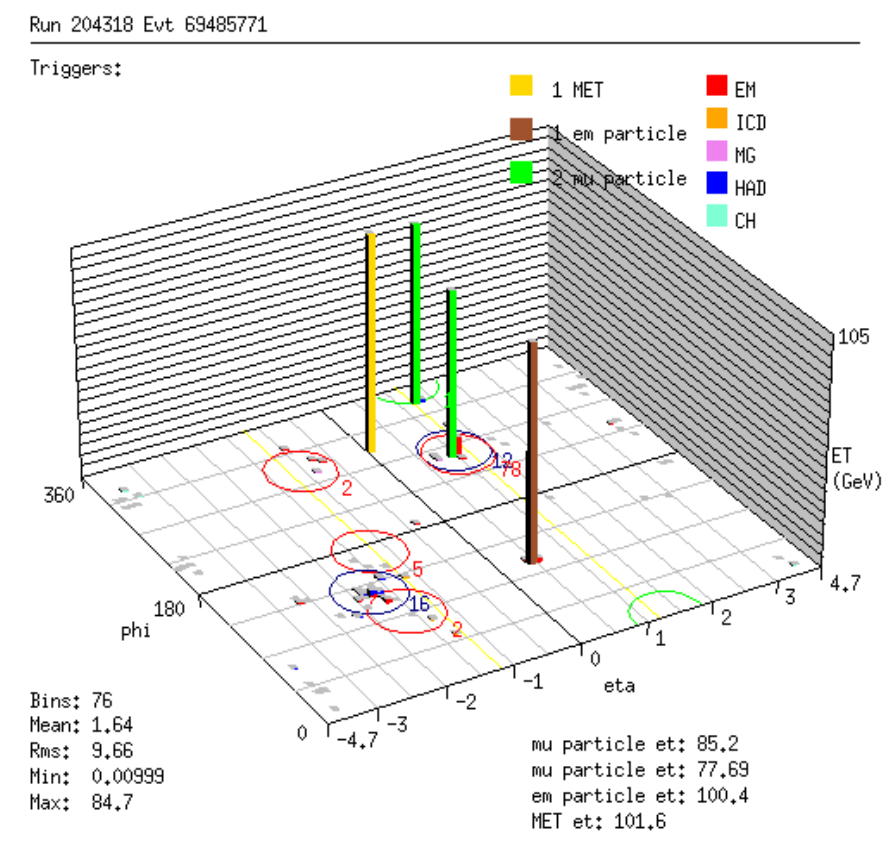

(c) Lego Plot

Figure E.7: Event View: Run Number - 204318 Event Number - 69485771 


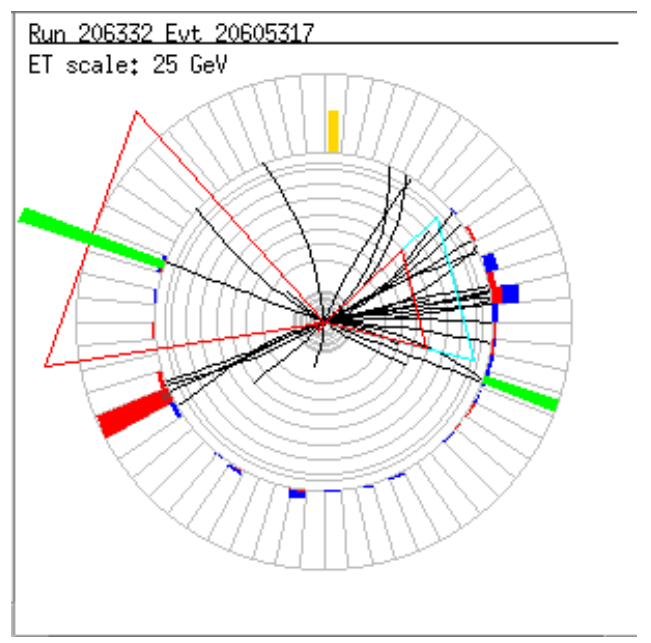

(a) $r-\phi$ view

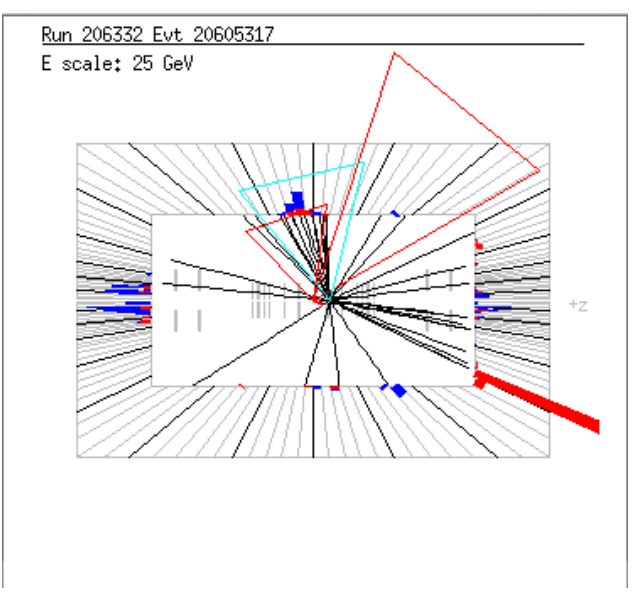

(b) $r-z$ view

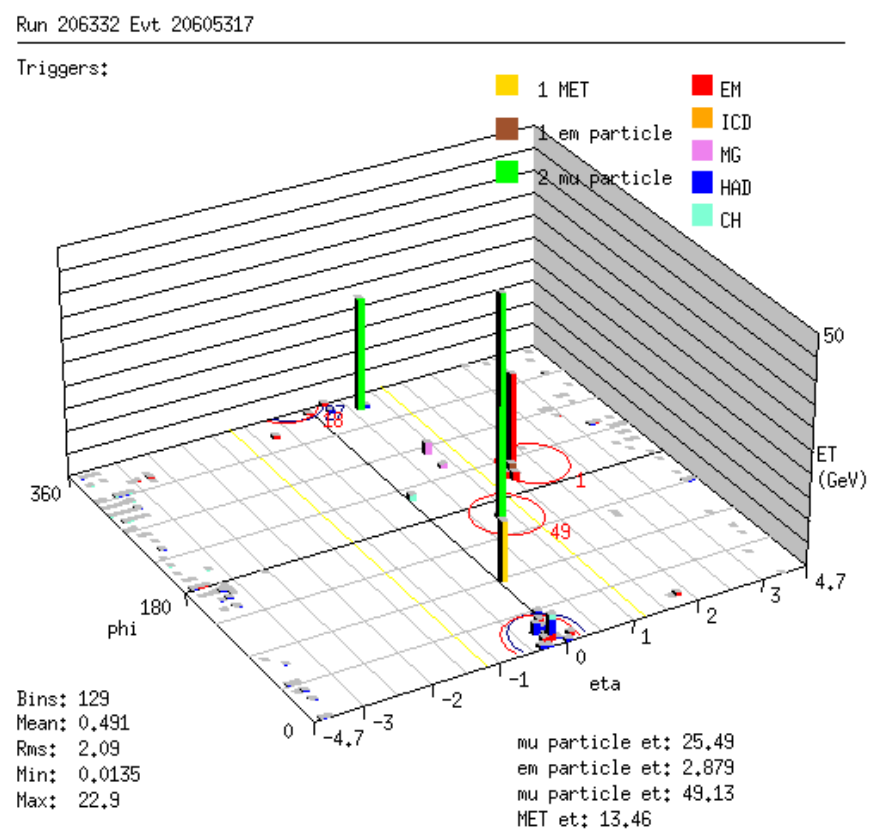

(c) Lego Plot

Figure E.8: Event View: Run Number - 206332 Event Number - 20605317 


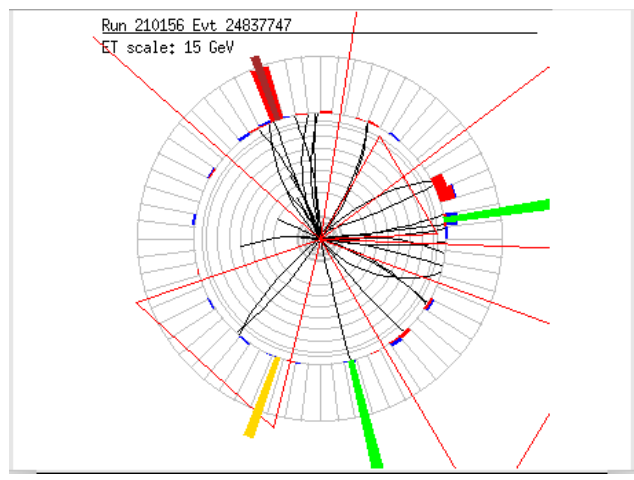

(a) $r-\phi$ view

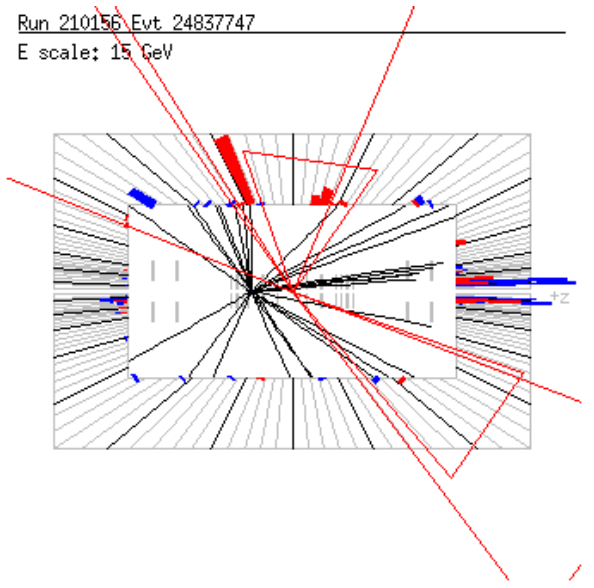

(b) $r-z$ view

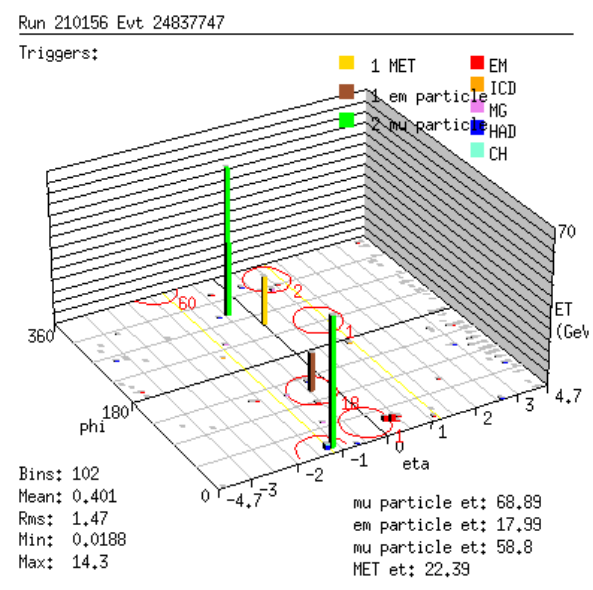

(c) Lego Plot

Figure E.9: Event View: Run Number - 210156 Event Number - 248377216 


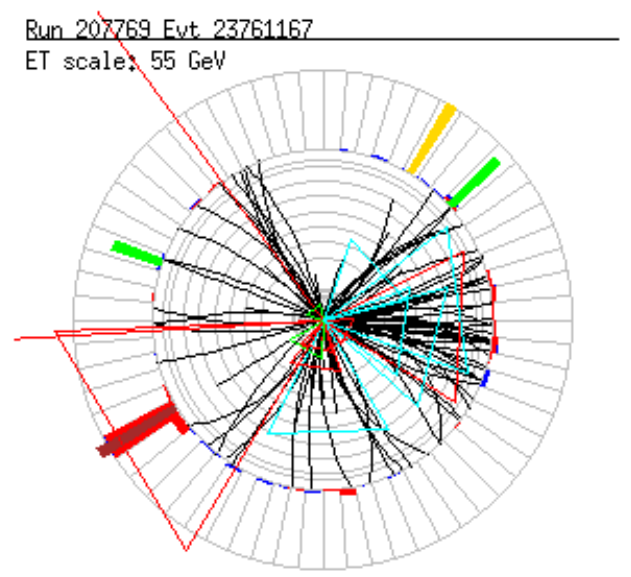

(a) $r-\phi$ view

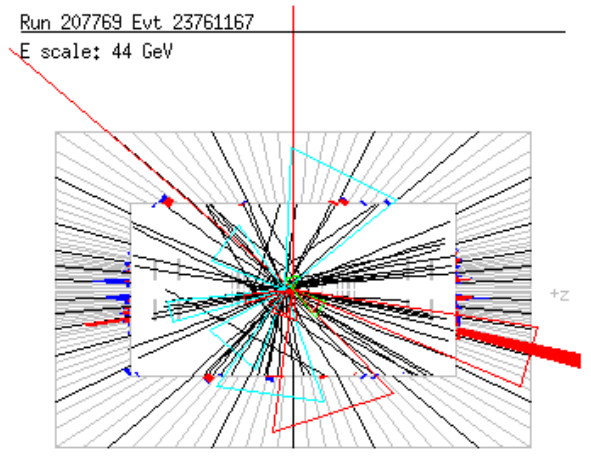

(b) $r-z$ view

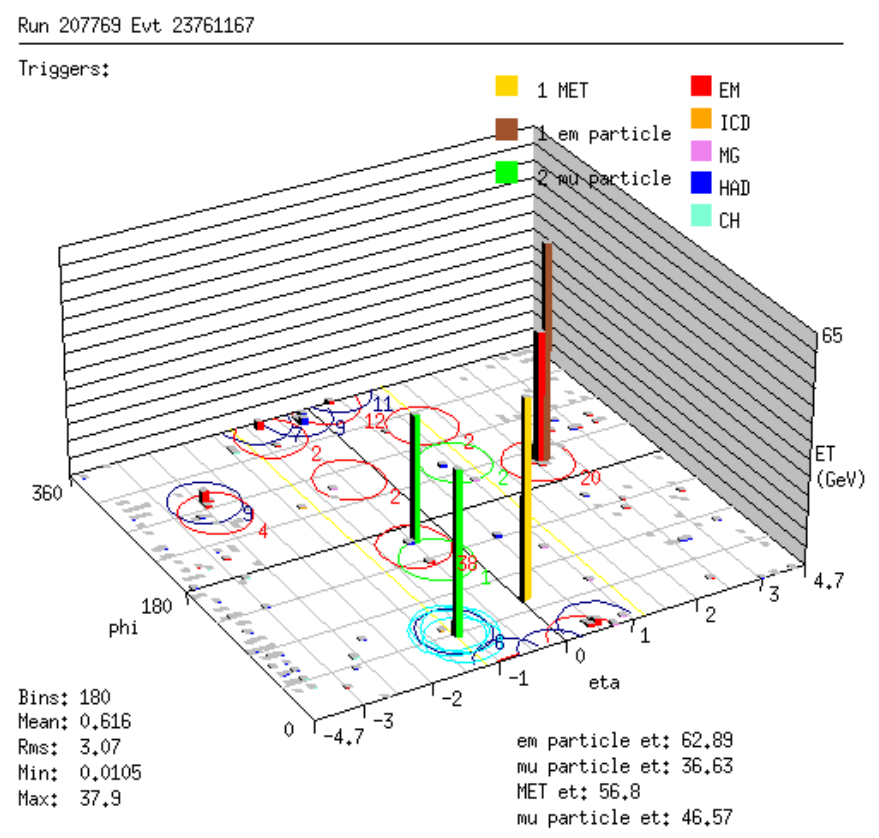

(c) Lego Plot

Figure E.10: Event View: Run Number - 207769 Event Number - 23761167 


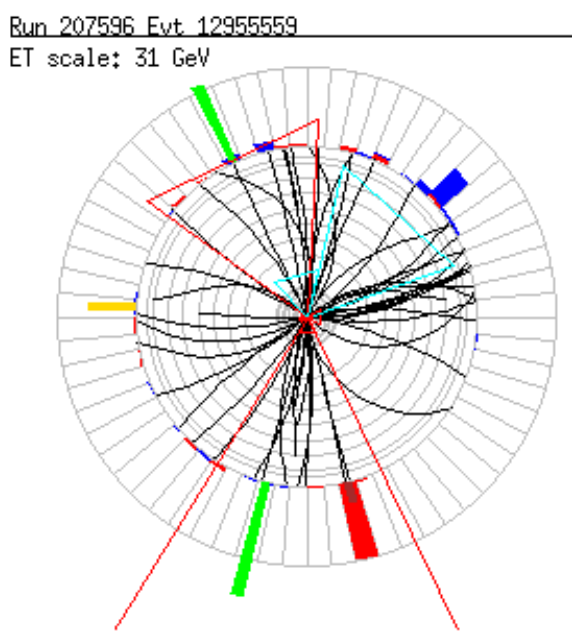

(a) $r-\phi$ view

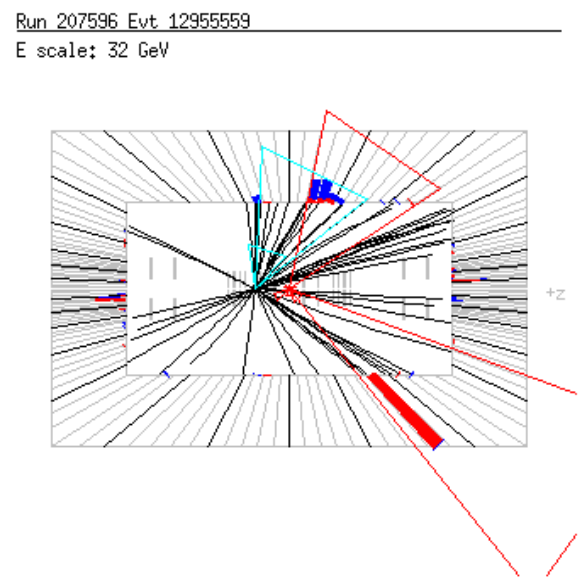

(b) $r-z$ view

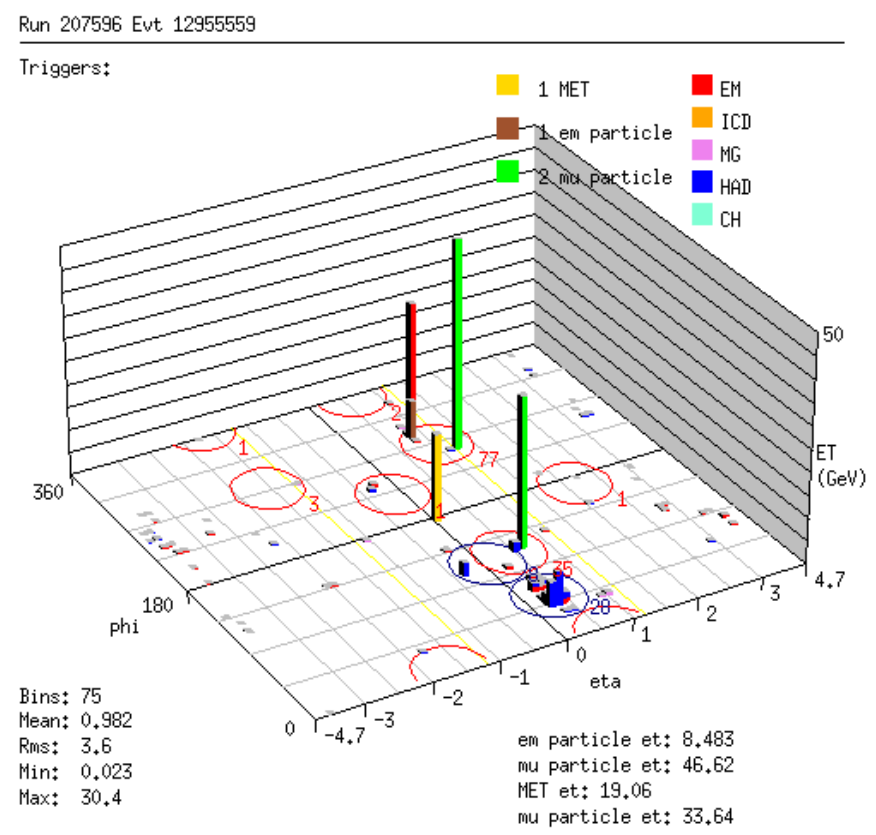

(c) Lego Plot

Figure E.11: Event View: Run Number - 207596 Event Number - 12955559 
Run 188371 Evt 23177216

E scale: 29 GeV

Run 188371 Evt 23177216

ET scale: $30 \mathrm{GeV}$

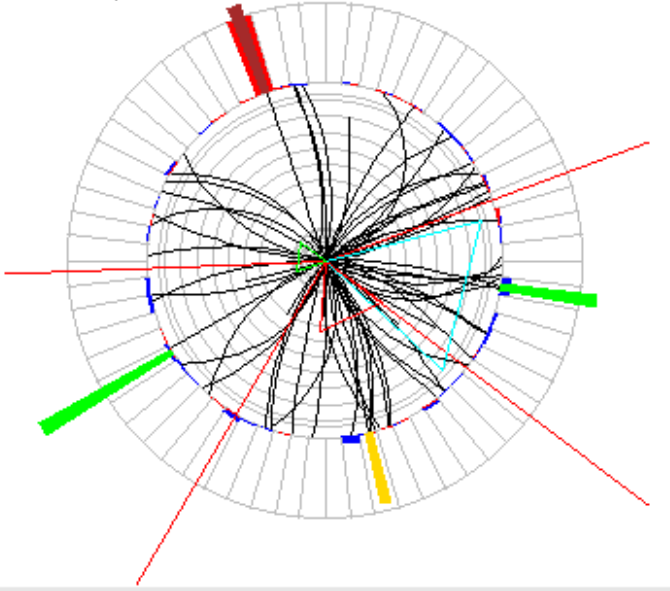

(a) $r-\phi$ view

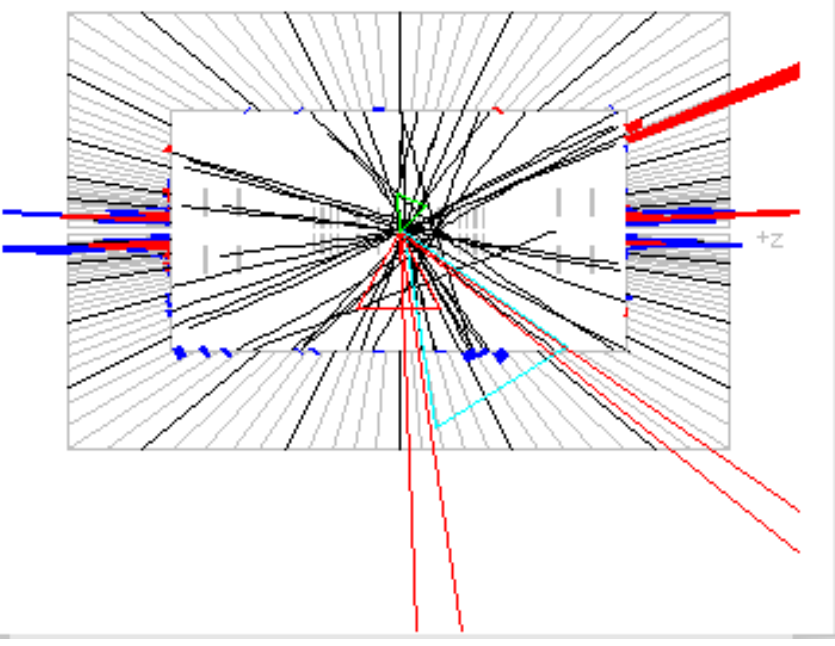

(b) $r-z$ view

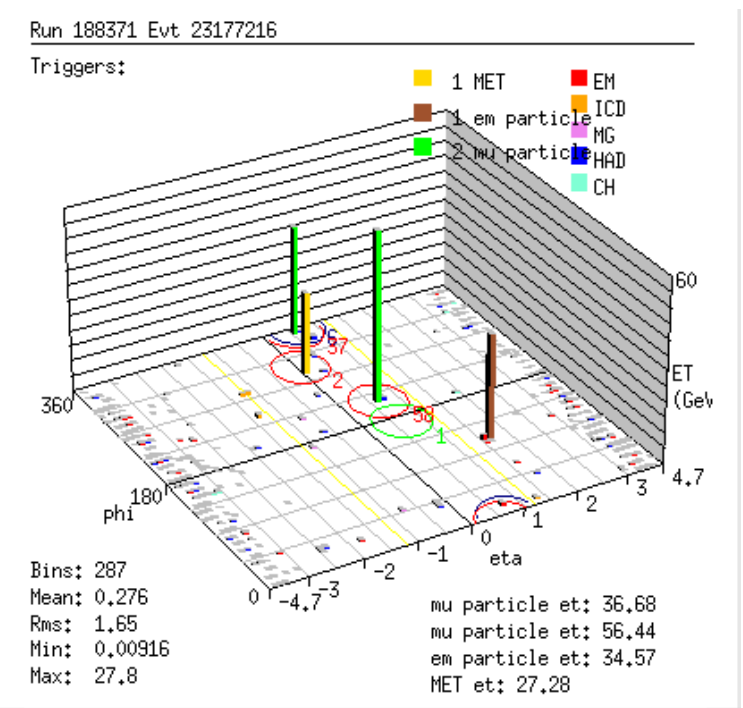

(c) Lego Plot

Figure E.12: Event View: Run Number - 188371 Event Number - 23177216 
Bun 192868 Eut 66474158

ET scale $\$ 23 \mathrm{GeV}$

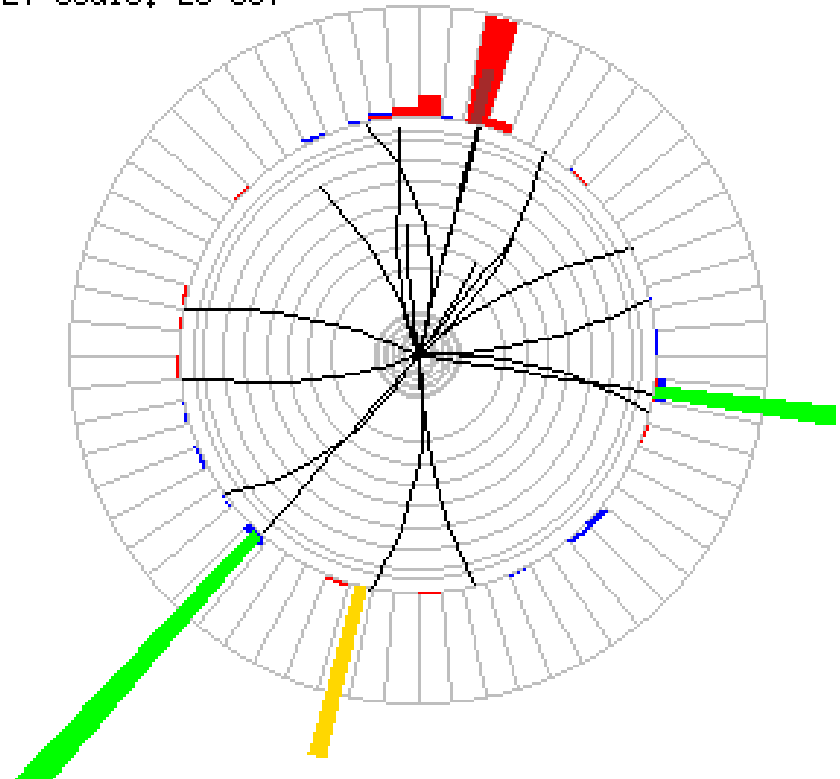

(a) $r-\phi$ view
Bun 192868 Eut 66474158

E scale* $21 \mathrm{GeV}$

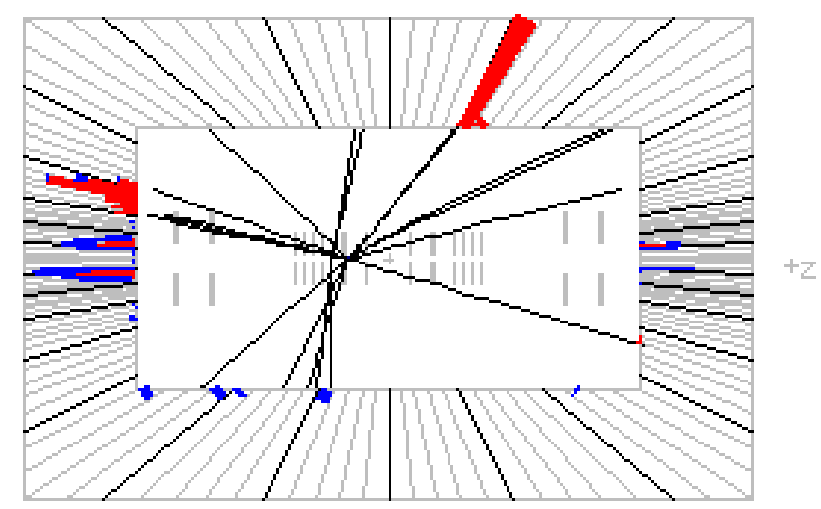

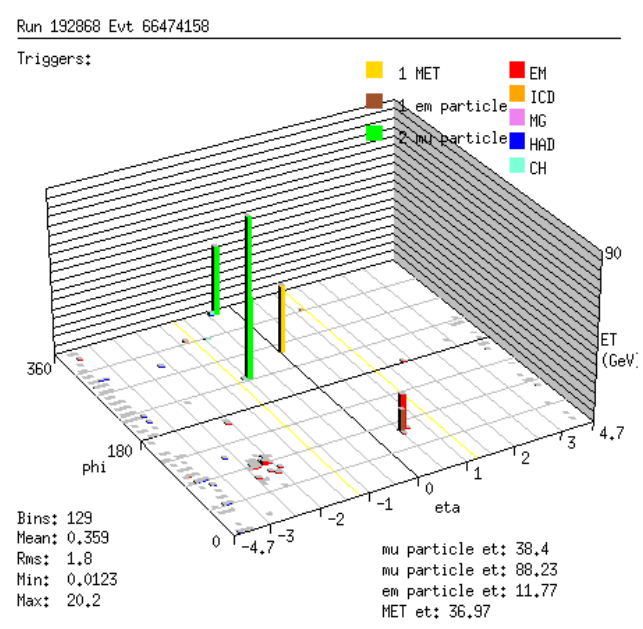

(c) Lego Plot

Figure E.13: Event View: Run Number - 192868 Event Number - 66474158 


\section{BIBLIOGRAPHY}


[1] S. Eidelman et Al., Review of particle physics, Phys. Lett. B, 592 (2004). xiv, 96

[2] G. Kane, Modern Elementary Particle Physics: The Fundamental Particles and Forces, Perseus Publishing, Cambridge, 1993. 1, 3, 4

[3] UA1 Collaboration, Experimental observation of isolated large transverse energy electrons with associated missing energy at $\sqrt{s}=540$ GeV, Phys. Lett., B122 (1983). 2, 18

[4] UA2 Collaboration, Observation of single isolated electron with high transverse momentum in events with missing transverse energy at the cern $\bar{p} p$ collider., Phys. Lett., B122 (1983). 2, 18

[5] UA1 Collaboration, Experimental observation of lepton pairs of invariant mass around 95 $\mathrm{GeV} / \mathrm{c}^{2}$., Phys. Lett., B126 (1983). 2, 18

[6] UA2 Collaboration, Evidence for $Z^{0} \rightarrow e^{+} e^{-}$at the cern $\bar{p} p$ collider., Phys. Lett., B129 (1983). 2,18

[7] R. P. Feynman, QED. THE STRANGE THEORY OF LIGHT AND MATTER, University Press, Princeton, 1985. Princeton, Usa: Univ. Pr. ( 1985) 158 P. ( Alix G. Mautner Memorial Lectures). 2

[8] E. Noether, Invariant variation problems, Transport Theory and Statistical Physics, 1 (1971). 3

[9] K. Hagiwara, R. Peccei, K. Hikasa, and D. Zeppenfeld, Probing the weak boson sector in $e^{+} e^{-} \rightarrow W^{+} W^{-}$., Nucl. Phys. B, 282 (1987). 4

[10] V. Barger and R. Phillips, Collider Physics, Perseus Publishing, Cambridge, 1996. 5

[11] D. H. Perkins, Introduction to High Energy Physics, Cambridge University Press, Cambridge, 2000. 5,9

[12] J. Thompson, Introduction to colliding beams at Fermilab, FERMILAB-TM-1909, (1994). 5, 23

[13] J. D. JACKson, Classic Electrodynamics, John Wiley and Sons, Inc., New York, 1998. 6

[14] S. Klimenko, J. Konigsberg, And T. M. Liss, Averaging of the inelastic cross sections measured by the cdf and the e811 experiments, FERMILAB-FN-0741, (2003). 8

[15] B. R. Roe, Probability and Statistics in Experimental Physics, Springer, New York, 2001. 10

[16] B. Аввот ет AL., Studies of $W W$ and $W Z$ production and limits on anomalous $W W \gamma$ and $W W Z$ couplings, Phys. Rev. D, 60 (1999). 10

[17] V. Abazov et AL., Production of $W Z$ events in $p \bar{p}$ collisions at $\sqrt{s}=1.96 \mathrm{Te} V$ and limits on anomalous $W W Z$ couplings, Phys. Rev. Lett., 95 (2005). 10, 83

[18] D. Acosta et Al., Search for $z z$ and $z w$ production in $p \bar{p}$ collisions at $\sqrt{s}=1.96$ tev, Phys. Rev. D, 71 (2005). 10

[19] S. Weinberg, A model of leptons, Phys. Rev. Lett., 19 (1967). 12, 14

[20] A. Salam, in Elementary Particle Theory, p. 367, Almqvist and Wiksell, Stockholm, 1968. 14

[21] F. Halzen and A. Martin, Quarks and Leptons, John Wiley \& Sons, Inc., 1994. 14

[22] T. Sjostrand, PYTHIA 5.7 and JETSET 7.4: Physics and manual, Comput. Phys. Commun., 82 (1994). 20

[23] T. Nunneman, http://www-clued0.fnal.gov/ nunne/cross-sectionsmcfm_cross-sections.html. 22, 118,129

[24] www.fnal.gov. 23 
[25] V. M. Abazov et AL., The upgraded do detector, Nucl. Instrum. Meth., A565 (2006), pp. 463-537. 32,53

[26] T. L. Edwards Et AL., Determination of the effective inelastic $p$ anti-p cross- section for the do run ii luminosity measurement, FERMILAB-TM-2278-E, (2004). 35

[27] V. Abazov et AL., DØ silicon microstrip tracker for Run IIA, in Proceedings of Como 2001, World Scientific, 2002. 39

[28] D. Lincoln, New technologies in the d0 central tracker upgrade, Nucl. Instrum. Meth., A379 (1996), pp. $424-426.42$

[29] M. Adams et AL., A new detector technique using triangular scintillating strips to measure the position of minimum ionizing particles, Nucl. Instrum. Meth., A378 (1996), pp. 131-142. 42

[30] A. Gordeev et AL., Technical design report of the forward preshower detector for the DØ upgrade., DØ Note, 3445 (1998). 42

[31] T. FerBel, Experimental Techniques in High Energy Physics, Addison-Wesley, 1978. 48

[32] B. BALdin ET AL., Technical design report of the central muon system, DØ Note, 3365 (1997). 53

[33] G. C. Blazey, The d0 run ii trigger, To be published in the proceedings of 10th IEEE Real-Time Computer Applications in Nuclear, Particle and Plasma Physics (RT 97), Beaune, France, 22-26 Sep 1997, (1997). 57

[34] M. Abolins Eт AL., www.pa.msu.edu/hep/d0/ftp/l1/framework/l1fw_tdr_05june98.txt. 57

[35] J. T. Linnemann, The do level 2 trigger, Int. J. Mod. Phys., A16S1C (2001), pp. 1166-1168. 57

[36] D. Edmunds ET AL., www.ps.msu.edu/hep/d0/ftp/l2/overview/globaltdr/global_tdr.ps. 57

[37] A. Boehnlien Et AL., Description of the DØ L3 trigger software components., DØ Note, 3630 (1999). 57

[38] S. SNYDER, httt://www.-d0.fnal.gov/d0dist/dist/packages/coor/devel/doc/coorover.ps. 57

[39] R. Angstadt Et AL., The DØ L3 trigger data acquisition system., IEEE Transactions on Nuclear Science, 51 (2004). 63

[40] VITA VME Technology, http://www.vita.com/vmefaq.html. 63

[41] Cisco Systems, http://www.cisco.com/. 63

[42] J. Kowalkowski ET AL., D0 offline reconstruction and analysis control framework, Prepared for International Conference on Computing in High- Energy Physics and Nuclear Physics (CHEP 2000), Padova, Italy, 7-11 Feb 2000, (2000). 64

[43] A. Khanov, Htf: Histograming method for finding tracks., DØ Note, 3778 (2000). 64

[44] G. Borisov, www-d0.fnal.gov/global_tracking/talks/20030228/talk-adm-030228.ps. 64

[45] H. Greenlee, The dø kalman track fit., DØ Note, 4303 (2004). 64

[46] A. Garcia-Bellido et al., Primary vertex certification p14., DØ Note, 4320 (2004). 67

[47] A. Schwartzman and M. Narain, Probabilistic primary vertex selection, DØ Note, 4042 (2002). 67

[48] F. Fleuret et AL., http://www-d0.fnal.gov/d0dist/dist/releases/p1\%.09.00/em_analyze/doc/EMAnalyze_doc.ps. 68 
[49] G. C. Blazey Et Al., Run ii jet physics, hep-ex/0005012, (2000). 70

[50] B. Andrieu and E. Busato, Jet algorithms in the DØ Run II software: Description and users guide, DØ Note, 4457 (2005). 70

[51] P. Calfayan et Al., Muon identification certification for p17 data, DØ Note, 5157 (2006). 74, 82, $83,89,92,93$

[52] http://www-d0.fnal.gov/run2physics/cs/index.html. 76

[53] http://www-d0.fnal.gov/run2physics/cs/index.html. 78

[54] http://www-d0.fnal.gov/run2physics/cs/caf/. 81

[55] http://www-d0.fnal.gov/phys_id/emid/d0_private/emid.html. 81

[56] http://www-d0.fnal.gov/computing/algorithms/muon/muon_algo.html. 81

[57] https://plone4.fnal.gov/P1/DoWiki/mu_id/muo_cert. 83

[58] G. Hesketh, https://plone4.fnal.gov/P1/DoWiki/caf/wz_cafreco/. 84

[59] A. Alton, A. Askew, Y. Maravin, ET Al., Study of $Z+\gamma$ events in DØRun II data, DØ Note, 4488 (2005). 85

[60] J. Hays, J. Mitrevski, C. Schwanenberger, and T. Toole, Single electron efficiencies in P17 data and monte-carlo, DØ Note, 5025 (2006). 89, 92

[61] https://plone4.fnal.gov/p1/d0wiki/tsg/caftrigger/dietriggers. 92, 141

[62] A. Harel, Jet ID optimization, DØ Note, 4919 (2005). 99

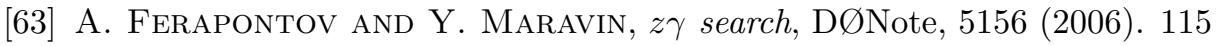

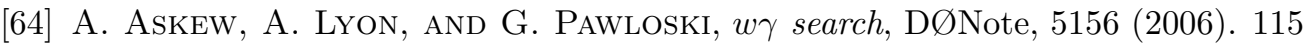

